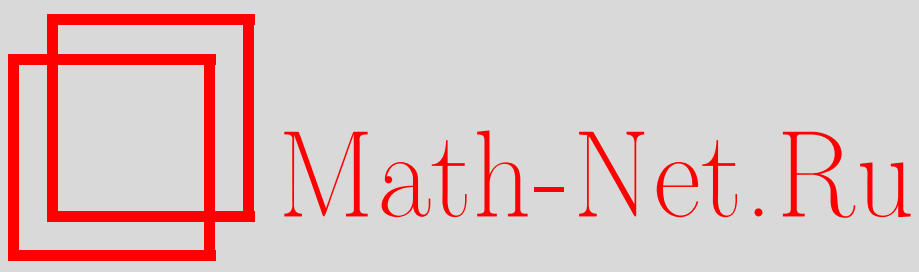

С. А. Кудрявцев, Классификация трехмерных исключительных логканонических гиперповерхностных особенностей. І, Из6. РАН. Сер. матем., 2002, том 66, выпуск $5,83-170$

DOI: https://doi.org/10.4213/im403

Использование Общероссийского математического портала Math-Net.Ru подразумевает, что вы прочитали и согласны с пользовательским соглашением

http://www.mathnet.ru/rus/agreement

Параметры загрузки:

IP : 3.80 .181 .102

26 апреля 2023 г., 14:26:55 
УДК 512.7

\author{
С. А. Кудрявцев
}

\title{
Классификация трехмерных исключительных логканонических гиперповерхностных особенностей. I
}

\begin{abstract}
Описаны трехмерные исключительные строго логканонические гиперповерхностные особенности и дана подробная классификация трехмерных исключительных канонических гиперповерхностных особенностей при условии хорошей определенности.

Библиографоя: 27 наименований.
\end{abstract}

\section{Введение}

Одна из основных проблем бирациональной геометрии, возникающая в логпрограмме минимальных моделей, - это изучение полученных экстремальных стягиваний (особенностей), т.е. стягиваний $f:\left(X, D_{X}\right) \rightarrow\left(X^{\prime}, D_{X^{\prime}}\right)$, где дивизор $-\left(K_{X}+D_{X}\right) f$-обилен и $\rho\left(X / X^{\prime}\right)=1$. В трехмерном случае эта проблема остается нерешенной, кроме частного случая классификации неособых многообразий но, полученной В. А. Исковских более 20 лет назад. Одна из основных сложностей при решении данной проблемы - это отсутствие геометрической классификации особенностей. Первым шагом при построении классификации гладких многообразий Фано является нахождение “хорошего” элемента в антиканонической линейной системе $\left|-K_{X}\right|$. Наличие "хорошего" элемента для экстремального стягивания уже позволяет увидеть структуру стягивания (пример 0.2). Поэтому основную проблему, которой и будет посвяшена настояшая работа, можно сформулировать следующим образом.

ПРоБлЕмА 0.1. Найти “хороший” элемент в кратной антиканонической линейной системе для экстремального стягивания (особенности).

Пример 0.2. 1) Рассмотрим малое экстремальное стягивание трехмерного терминального многообразия. Тогда существование дивизора с дювалевскими особенностями в линейной системе $\left|-2 K_{X}\right|$ влечет существование флипа [6].

2) Если экстремальное стягивание трехмерного терминального многообразия является расслоением на коники, то существование дивизора с дювалевскими особенностями в антиканонической линейной системе $\left|-K_{X}\right|$ позволяет сразу получить полную локальную классификацию [15].

Наличие “хорошего” элемента тесно связано и с другими проблемами, например с автоморфизмами К3-поверхностей [24, п. 1.12] и с индексами горенштейновости строго логканонических особенностей (см. пример 1.8).

Работа выполнена при частичной поддержке грантов РФФИ (грант № 02-01-00441), Ведущих научных школ (грант № 00-15-96085) и INTAS-OPEN (грант № 2000\#269).

(C) С. А. Кудрявцев, 2002 
Сравнительно недавно В. В. Шокуров в работах [23], [24] предложил путь решения проблемы 0.1. Он состоит из двух частей:

1) индуктивного перехода от многообразий размерности $n$ к многообразиям размерности $n-1$;

2) разделения стягиваний на исключительные и неисключительные.

Сначала необходимо найти такой дивизор $S$ на $X$ или на некотором раздутии $X$, что пара $\left(S, \operatorname{Diff}_{S}\left(D_{X}\right)\right)$ будет логтерминальной по Кавамате и дивизор $-\left(K_{S}+\right.$ $\left.\operatorname{Diff}_{S}\left(D_{X}\right)\right)$ окажется обильным. Тогда "хороший" дивизор для этой пары продолжается до "хорошего" дивизора на всем многообразии $X$.

Явление исключительности при изучении экстремальных стягиваний (особенностей) состоит в следуюшем:

1) если экстремальное стягивание неисключительное, то для него можно найти "хороший" дивизор из линейной системы $\left|-n K_{X}\right|$ для небольших значений $n$, например для двумерных логканонических особенностей $n \in\{1,2\}$ [23, пример 5.2], а в трехмерном случае $n \in\{1,2,3,4,6\}$ [24, теорема 7.1];

2) исключительные стягивания являются "ограниченными", и можно дать их подробное описание; например, в настояшей работе проверено, что трехмерные исключительные гиперповерхностные особенности имеют конечное число типов.

Регулярные, т.е. неисключительные экстремальные стягивания (особенности), вообще говоря, не поддаются детальной классификации уже в размерности три (например, в работе [13] показано, что невозможно перечислить уравнения нормальных форм всех трехмерных терминальных гиперповерхностных особенностей, т.е. самых простых трехмерных особенностей). С другой стороны, наличие дополнения минимального индекса позволяет разбить экстремальные стягивания (особенности) на семейства с общими свойствами.

Исключительные стягивания (особенности) поддаются детальной классификации, хотя и могут иметь большой индекс дополняемости.

В статье [17] было показано, что исключительность сохраняется при индуктивном переходе и, наоборот, из исключительности пары $\left(S, \operatorname{Diff}_{S}\left(D_{X}\right)\right)$ следует исключительность $\left(X / Z, D_{X}\right)$. Тем самым, стало возможным говорить об индуктивном методе классификации алгебраических многообразий.

Настояшая статья посвящена применению этого метода к гиперповерхностным особенностям. В работе классифицируются трехмерные исключительные канонические гиперповерхностные особенности. Для этого описываются соответствующие квазиоднородные части, потом строятся взвешенные чисто логтерминальные раздутия и исследуются на исключительность полученные логповерхности дель Пецю. В частности, находится минимальный индекс дополняемости. Также получена классификация трехмерных исключительных строго логканонических гиперповерхностных особенностей.

ОСНОВНАЯ ТЕОРЕМА. Пусть $(X, 0) \subset\left(\mathbb{C}^{4}, 0\right)$ - трехмерная исключительная каноническая (соответственно, строго логканоническая) гиперповерхностная особенность, заданная многочленом $f$. Тогда существуют биголоморфная замена координат $\psi:\left(\mathbb{C}^{4}, 0\right) \rightarrow\left(\mathbb{C}_{t, z, x, y}^{4}, 0\right)$ и единственный примитивный вектор $\mathbf{p} \in N_{\mathbb{R}}$ такие, что выполняется одно из следующих условий. 
1) Квазиоднородный многочлен $\tilde{f}_{\mathbf{p}}=(f \circ \psi)_{\mathbf{p}}$ задает исключительную каноническую (соответственно, строго логканоническую и каноническую вне $\mathbf{0}$ ) особенность $\left(X_{\mathbf{p}}, 0\right) \subset\left(\mathbb{C}_{t, z, x, y}^{4}, 0\right) ;$ в этом случае $\mathbf{p}$-раздутие $\mathbb{C}^{4}$ индуцирует иисто логтерминальные раздутия $\varphi:(Y, E) \rightarrow(X, 0)$ и $\varphi_{\mathbf{p}}:\left(Y_{\mathbf{p}}, E_{\mathbf{p}}\right) \rightarrow$ $\left(X_{\mathbf{p}}, 0\right)$, причем $\left(E, \operatorname{Diff}_{E}(0)\right)=\left(E_{\mathbf{p}}, \operatorname{Diff}_{E_{\mathbf{p}}}(0)\right)$, т.е. эти особенности имеют один и тот же тип, в частности, одинаковый индекс дополняемости. Канонические особенности, удовлетворяющие условию хорошей определенности: $\operatorname{Diff}_{E / \mathbb{P}(\mathbf{p})}(0)=0$, классифицированы в теоремах $3.24,3.29$, в таблицах $\S 4$, где указаны $\tilde{f}_{\mathbf{p}},\left(E, \operatorname{Diff}_{E}(0)\right)$, минимальный индекс дополняемости, и в работе [27]. Канонические особенности, не удовлетворяющие условию хорошей определенности, классифичировань в работе [27]. Строго логканонические и канонические вне 0 квазиоднородные особенности согласно теореме 2.13 всегда являются исключительными (в любой размерности). В трехмерном случае $\left(E, \operatorname{Diff}_{E}(0)\right)=\left(\tilde{f}_{\mathbf{p}} \subset \mathbb{P}(\mathbf{p}), 0\right)-\mathrm{K} 3$-поверхность с дювалевскими особенностями и особенность $(X, 0)$ 1-дополняема.

2) Квазиоднородная часть имеет вид $\tilde{f}_{\mathbf{p}}=t^{3}+g_{2}^{2}(z, x, y)$, где $g_{2}-$ неприводимый однородный многочлен степени 2. Для этого случая в теоремах 3.3 u 3.5 построено чисто логтерминальное раздутие и получена аналогичная классификация в зависимости от вида струй $\tilde{f}_{5}$ и $\tilde{f}_{6}$.

Для невырожденных строго логканонических особенностей было доказано, что они исключительны тогда и только тогда, когда они имеют чисто эллиптический тип $(0, d-1)$, где $d$ - размерность особенности [4]. Для невырожденных строго логканонических особенностей всегда можно выделить квазиоднородную логканоническую часть $[19$, теорема 3.5$]$. Поэтому в наших терминах эти особенности исключительны тогда и только тогда, когда квазиоднородная логканоническая часть задает каноническую вне 0 особенность (следствие 2.18). Исходя из этого, несложно получить список трехмерных исключительных строго логканонических особенностей. В настоящей работе он не приводится. Для изолированных особенностей список приведен в [26]. Этот список соответствует 95 семействам взвешенных К3-поверхностей, полученных Флетчером и Ридом в [2], [21, теорема 4.5]. Действительно, исключительный дивизор чисто логтерминального раздутия - это К3-поверхность.

Статья организована следуюшим образом: в $\S 1$ собраны основные сведения и предварительные результаты, в $§ 2$ рассмотрены квазиоднородные особенности, в $\S 3$ доказана основная теорема и приведены пояснения к исследованию трехмерных канонических особенностей на исключительность, в $\S 4$ приведены итоговые таблищы для особенностей с ненулевой квадратичной частью.

Автор очень признателен В. А. Исковских, Ю. Г. Прохорову, В. В. Шокурову за ценные советы и постоянный интерес к работе.

\section{§ 1. Предварительные сведения и результаты}

Все многообразия рассматриваются над полем комплексных чисел $\mathbb{C}$. Основные определения, обозначения и понятия, использованные в статье, приведены в [7], [8], [20]. Строго логканоническими особенностями называются логканонические, не чисто логтерминальные особенности. 
ОПРЕДЕЛЕНИЕ 1.1. $\mathbb{Q}$-дивизор $D$ называется дивизором со стандартнылми коэффищиентами, если каждый его коэффищиент имеет вид $(1-1 / m)$ или 1 , где $m \in \mathbb{N}$.

ОПРЕДЕЛЕНИЕ 1.2. Пусть $(X / Z \ni P, D)$ - стягивание многообразий. $\mathbb{Q}$-дополнением для этого стягивания называется эффективный $\mathbb{Q}$-дивизор $D^{\prime}$ такой, что $D^{\prime} \geqslant D$, дивизор $K_{X}+D^{\prime}$ логканоничен и $K_{X}+D^{\prime} \sim_{\mathbb{Q}} 0$.

ОПРЕДЕЛЕНИЕ 1.3. Пусть $X-$ нормальное многообразие, а дивизор $D=S+B$ является субгранищей, где $S$ - его целая эффективная часть и $\llcorner B\lrcorner \leqslant 0$. Дивизор $K_{X}+D$ называется $n$-дополняемы.м, если существует $\mathbb{Q}$-дивизор $D^{+}$, называемый $n$-дополнением, который удовлетворяет следуюшим требованиям:

1) $n\left(K_{X}+D^{+}\right) \sim 0$

2) дивизор $K_{X}+D^{+}$логканоничен;

3) $n D^{+} \geqslant n S+\llcorner(n+1) B\lrcorner$.

Инвариантность дополнений относительно логпрограммы минимальных моделей и их индуктивные свойства показаны в следующих трех теоремах.

Теорема 1.4 [23, лемма 5.4]. Пусть $f: X \rightarrow Z$ - бирациональное стягивание многообразий и дивизор $D$ - субграница. Если пара $K_{X}+D$ n-дополняема, mо пара $K_{Z}+f_{*} D$ mакже $n$-дополняема.

Теорема 1.5 [24, лемма 2.13]. Пусть $f: X \rightarrow Z$ - бирациональное стягивание многообразий и дивизор $D$ - субгранича. Предположим, что дивизор $K_{X}+D \quad f$-численно эффективен и дивизор $f_{*} D$ имеет стандартные коэффициенты. Если пара $K_{Z}+f_{*} D$ n-дополняема, то пара $K_{X}+D$ такәе n-дополняема.

ТЕОРема 1.6 [16, лемма 2.1]. Пусть $(X, S)$ - чисто логтерминальная пара с приведенным дивизором $S \neq 0$, и пусть $f: X \rightarrow Z$ - проективный морфизм такой, что дивизор - $\left(K_{X}+S\right) \quad f$-численно әффективен и $f$-обвемен. Тогда для заданного $n$-дополнения $K_{S}+\operatorname{Diff}_{S}(0)^{+}$nары $K_{S}+\operatorname{Diff}_{S}(0)$ в окрестности слоя $f$, пересекающего $S$, существует $n$-дополнение $K_{X}+S+B$ пары $K_{X}+S$ maкое, что $\operatorname{Diff}_{S}(0)^{+}=\operatorname{Diff}_{S}(B)$.

ОПРЕДЕЛЕНИЕ 1.7. Пусть $(X / Z \ni P, D)$ - стяГивание многообразий. При $\operatorname{dim} Z \neq 0$ стягивание называется исключительным, если для любого $\mathbb{Q}$-дополнения $D^{\prime}$ существует не более одного дивизора $E$ (не обязательно исключительного) такого, что $a\left(E, D^{\prime}\right)=-1$. При $\operatorname{dim} Z=0$ логмногообразие назьвается исключительнылм, если для любого $\mathbb{Q}$-дополнения $D^{\prime}$ пара $\left(X, D^{\prime}\right)$ логтерминальна по Кавамате.

ПРИмЕР 1.8. 1) Если для особенности $(X \ni P)$ существует эффективный дивизор $H$ такой, что пара $(X, H)$ логканонична и $\llcorner H\lrcorner \neq 0$, то особенность неисключительна. Поэтому трехмерная терминальная особенность неисключительна, так как в антиканонической линейной системе $\left|-K_{X}\right|$ существует дивизор с дювалевскими особенностями $[22$, п. 6.4$]$. Для четырехмерных терминальных особенностей это уже не верно [10]. 
2) Для строго логканонической исключительной особенности минимальный индекс дополняемости совпадает с индексом горенштейновости особенности $[11$, следствие 1.10]. Для трехмерных логканонических особенностей минимальньй индекс дополняемости ограничен [24, теорема 7.1]. Гипотетически он не более 66. Для трехмерных исключительных строго логканонических особенностей это доказано в работах [5] и [3]. Для неисключительных неизолированных особенностей индекс горенштейновости не ограничен [3, пример 5.1].

ОПРЕДЕЛЕНИЕ 1.9. Пусть $(X \ni P)$ - логканоническая особенность, и пусть $f: Y \rightarrow X$ - некоторое ее раздутие. Предположим, что исключительное множество $f$ состоит только из дивизоров: $\operatorname{Exc}(f)=E$. Тогда $f:(Y, E) \rightarrow(X \ni P)$ называется чисто логтерминальным раздутием, если дивизор $K_{Y}+E$ чисто логтерминален и $-E f$-обилен.

ОПРЕДЕЛЕНИЕ 1.10. Пусть $(X \ni P)$ - логканоническая особенность, и пусть $f: Y \rightarrow X$ - некоторое ее раздутие. Предположим, что исключительное множество $f$ состоит только из дивизоров: $\operatorname{Exc}(f)=E$. Тогда $f:(Y, E) \rightarrow(X \ni P)$ назьвается логканоническим раздутием, если дивизор $K_{Y}+E$ логканоничен и $-E f$-обилен.

ЗАмЕчАнИЕ 1.11. В определениях 1.9 и 1.10 неявно требуется, чтобы дивизор $E$ был $\mathbb{Q}$-картье. Отсюда многообразие $Y$ имеет $\mathbb{Q}$-горенштейновые особенности.

ЗАмЕчАниЕ 1.12. В обозначениях определения 1.9 имеем следующие свойства чисто логтерминальных раздутий:

1) если многообразие $X$ имеет логтерминальные особенности, то чисто логтерминальное раздутие всегда существует $[11$, теорема 1.5$]$ и дивизор $-\left(K_{Y}+E\right)$ $f$-обилен;

2) если многообразие $X$ имеет строго логканонические особенности, то для исключительных особенностей чисто логтерминальное раздутие всегда существует [11, теорема 1.9$]$ и $a(E, 0)=-1$;

3) если многообразие $X$ является $\mathbb{Q}$-факториальным, то $Y$ также $\mathbb{Q}$-факториально и $\rho(Y / X)=1[17$, замечание 2.2], отсюда следует, что в определении 1.9 для $\mathbb{Q}$-факториального случая можно не требовать относительной обильности дивизора $-E$, так как она всегда имеет место;

4) пусть $f_{i}:\left(Y_{i}, E_{i}\right) \rightarrow(X \ni P)$ - два чисто логтерминальных раздутия; если $E_{1}$ и $E_{2}$ определяют одно и то же дискретное нормирование поля функций $\mathscr{K}(X)$, то раздутия $f_{1}$ и $f_{2}$ изоморфны [17, замечание 2.2$]$;

$5)$ в силу обрашения присоединения дивизор $K_{E}+\operatorname{Diff}_{E}(0)$ логтерминален по Кавамате; любое его $n$-дополнение продолжается до $n$-дополнения $K_{Y}+E$, следовательно, $K_{X}$ также $n$-дополняем (см. теоремы 1.6 и 1.4 ).

ОПРЕДЕЛЕНИЕ 1.13. Логканоническая особенность $(X \ni P)$ называется $с л а$ бо исключительной, если для нее существует ровно одно чисто логтерминальное раздутие (с точностью до изоморфизма).

Следуюшие теоремы показывают индуктивный переход от $n$-мерной исключительной (слабо исключительной) логтерминальной особенности $(X \ni P)$ к един- 
ственному исключительному (слабо исключительному) логмногообразию Фано $\left(E, \operatorname{Diff}_{E}(0)\right)$ размерности $n-1$, которое наследует дополняемость, и наоборот.

Теорема 1.14 [17, теорема 4.9]. Пусть $(X \ni P)$ - логтерминальная особенность и $f:(Y, E) \rightarrow(X \ni P)$ - чисто логтерминальное раздутие. Тогда следуюшие утверждения әквивалентны:

1) особенность $(X \ni P)$ исключительна;

2) логмногообразие $\left(E, \operatorname{Diff}_{E}(0)\right)$ исключительно.

ТЕорема 1.15 [11, теорема 2.1]. Пусть $(X \ni P)$ - логтерминальная особенность и $f:(Y, E) \rightarrow(X \ni P)$ - чисто логтерминальное раздутие. Тогда следующие условия әквивалентнь:

1) $(X \ni P)$ - не слабо исключительная особенность;

2) существует әффективный $\mathbb{Q}$-дивизор $D \geqslant \operatorname{Diff}_{E}(0)$ такой, что $-\left(K_{E}+\right.$ $D)$ - обильный дивизор и пара $(E, D)$ не логтерминальна по Кавамате;

3) существует эффективный $\mathbb{Q}$-дивизор $D \geqslant \operatorname{Diff}_{E}(0)$ такой, что $-\left(K_{E}+\right.$ $D)$ - обильный дивизор и пара $(E, D)$ не логканонична.

Аналогично примеру слабо исключительной особенности (но не исключительной особенности), построенному в любой размерности [11, пример 2.4], можно построить пример исключительной особенности.

ПРимеР 1.16. Рассмотрим $n$-мерную каноническую гиперповерхностную особенность $\left(x_{1}^{n}+x_{2}^{n+1}+x_{3}^{n+1}+\cdots+x_{n+1}^{n+1}=0\right) \subset\left(\mathbb{C}^{n+1}, 0\right)$ и взвешенное раздутие $\mathbb{C}^{n+1}$ с весами $(n+1, n, n, \ldots, n)$, которое индуцирует чисто логтерминальное раздутие данной особенности. Используя основные теоремы о взвешенных пространствах [2], нетрудно вычислить полученное логмногообразие Фано $\left(E, \operatorname{Diff}_{E}(0)\right)($ см. также $\S 4)$ :

$$
\begin{aligned}
& \left(\tilde{x}_{1}+\tilde{x}_{2}^{n+1}+\cdots+\tilde{x}_{n+1}^{n+1} \subset \mathbb{P}(n+1,1, \ldots, 1), \frac{n-1}{n}\left\{\tilde{x}_{1}=0\right\}\right)= \\
& \quad=\left(\mathbb{P}^{n-1}, \frac{n-1}{n} Q_{n+1}\right)
\end{aligned}
$$

где $Q_{n+1}$ - гладкая гиперповерхность в $\mathbb{P}^{n-1}$ степени $n+1$. Тем же способом, что и в работе [10], несложно проверить, что для $n \geqslant 3$ полученное логмногообразие является исключительным. Дивизор $\frac{n-1}{n}\left\{\tilde{x}_{1}=0\right\}+\frac{1}{n}\left\{\tilde{x}_{2}=0\right\}$ дает $n$-дополнение минимального индекса. Отсюда по критерию исключительности 1.14 данная особенность исключительна и согласно замечанию $1.12 n$-дополняема.

ПРЕДЛОЖЕНИЕ 1.17. Исключительная особенность является слабо исключительной. Если существует логканоническое, но не чисто логтерминальное раздутие, то особенность неисключительна.

ДокАЗАТЕЛЬСТво. Первое утверждение в общем случае доказано в $[11$, следствие 1.12]. Второе утверждение содержится в доказательстве $[19$, предложение 2.4]. Действительно, пусть $f:(Y, E) \rightarrow(X \ni P)$ - логканоническое раздутие, но не чисто логтерминальное раздутие. Далее можно считать, что $(X \ni P)-$ логтерминальная особенность, иначе все доказано. Так как $-\left(K_{Y}+E\right) f$-обилен, 
то линейная система $\left|-n\left(K_{Y}+E\right)\right|$ очень обильна над $X$ для $n \gg 0$. Пусть $H \in\left|-n\left(K_{Y}+E\right)\right|-$ общий элемент. Положим $B=\frac{1}{n} f(H)$. По теореме Бертини дивизор $K_{Y}+E+\frac{1}{n} H$ логканоничен, но не чисто логтерминален. Так как $K_{Y}+E+\frac{1}{n} H \sim_{\mathbb{Q}} 0$, то пара $(X, B)$ логканонична и неисключительна.

Согласно [11, следствие 1.6, теорема 1.9] имеем

ПрЕДЛОЖЕНИЕ 1.18. Пусть $f:(Y, E) \rightarrow(X \ni P)$ - иисто логтерминальное раздутие логканонической особенности. Предположим, что $\operatorname{dim} f(E) \geqslant 1$. Если $(X \ni P)$ - логтерминальная особенность, то она не является слабо исключительной. Если $(X \ni P)$ - строго логканоническая особенность, то она является слабо исключительной, но не исключительной.

ПримеР 1.19 [23, пример 5.2]. Двумерные логтерминальные исключительные особенности-это особенности типов $\mathbb{E}_{6}, \mathbb{E}_{7}, \mathbb{E}_{8}$. Их чисто логтерминальные раздутия - это раздутие центральной вершины графа минимального разрешения. Соответствующие им одномерные логмногообразия Фано $\left(E, \operatorname{Diff}_{E}(0)\right)$ - это $\left(\mathbb{P}^{1}\right.$, $\left.\frac{n_{1}-1}{n_{1}} P_{1}+\frac{n_{2}-1}{n_{2}} P_{2}+\frac{n_{3}-1}{n_{3}} P_{3}\right)$, где $\left(n_{1}, n_{2}, n_{3}\right)=(2,3,3),(2,3,4),(2,3,5)$ соответственно.

ПримеР $1.20[20, \S 6]$. Двумерные исключительные строго логканонические исключительные особенности - это следуюшие особенности: простые эллиптические особенности, особенности типов $\widetilde{\mathbb{D}}_{4}, \widetilde{\mathbb{E}}_{6}, \widetilde{\mathbb{E}}_{7}, \widetilde{\mathbb{E}}_{8}$. Их чисто логтерминальные раздутия - это раздутие центральной вершины графа минимального разрешения. Соответствуюшие им одномерные логмногообразия $\left(E, \operatorname{Diff}_{E}(0)\right)$ - это гладкая эллиптическая кривая, $\left(\mathbb{P}^{1}, \frac{1}{2} P_{1}+\frac{1}{2} P_{2}+\frac{1}{2} P_{3}+\frac{1}{2} P_{4}\right)$ и $\left(\mathbb{P}^{1}, \frac{n_{1}-1}{n_{1}} P_{1}+\frac{n_{2}-1}{n_{2}} P_{2}+\right.$ $\left.\frac{n_{3}-1}{n_{3}} P_{3}\right)$, где $\left(n_{1}, n_{2}, n_{3}\right)=(3,3,3),(2,4,4),(2,3,6)$ соответственно.

Итак, для двумерных исключительных логтерминальных особенностей сушествует всего три типа индуцированных логмногообразий Фано, а для строго двумерных строго логканонических - пять типов. Подобный результат о конечности типов гипотетически верен для любой размерности. Для трехмерных гиперповерхностных особенностей это утверждение проверено в настоящей работе. Принципиальное отличие многомерных особенностей от двумерных особенностей состоит в том, что почти в каждом типе существует бесконечное число попарно не изоморфных особенностей.

ОПРЕДЕЛЕНИЕ 1.21. Напомним основную терминологию торической геометрии [14], которая используется далее. Пусть $N$ - свободная абелева группа $\mathbb{Z}^{n}$ и $M$ - ее двойственная решетка $\operatorname{Hom}_{\mathbb{Z}}(N, \mathbb{Z})$. Обозначим $N \otimes_{\mathbb{Z}} \mathbb{R}$ и $M \otimes_{\mathbb{Z}} \mathbb{R}$ через $N_{\mathbb{R}}$ и $M_{\mathbb{R}}$ соответственно. Имеем билинейное спаривание $\langle\rangle:, N_{\mathbb{R}} \times M_{\mathbb{R}} \rightarrow \mathbb{R}$. Пусть $\sigma$ - положительньй октант $\mathbb{R}_{\geqslant 0}^{n}$ решетки $N_{\mathbb{R}}=\mathbb{R}^{n}$ и $\sigma^{\vee}$ - его двойственньй октант. Тогда $\mathbb{C}^{n}$ является торическим многообразием, соответствующим конусу $\sigma$. Для веера $\Delta$ в $N$ соответствуюшее торическое многообразие обозначается через $T_{N}(\Delta)$. Пусть $\mathbf{p} \in N$ - примитивный вектор из одномерного конуса $\tau=\mathbb{R}_{\geqslant 0} \mathbf{p}$ в $\Delta ;$ тогда замыкание $\overline{\operatorname{orb}\left(\mathbb{R}_{\geqslant 0} \mathbf{p}\right)}$ обозначается через $D_{\mathbf{p}}$, где $D_{\mathbf{p}}$ - дивизор на многообразии $T_{N}(\Delta)$.

Пусть $(X, 0) \subset\left(\mathbb{C}^{n}, 0\right)$ - гиперповерхностная особенность, и пусть $\mathbf{p}=\left(p_{1}, \ldots\right.$ $\left.\ldots, p_{n}\right)$ - примитивный элемент в решетке $N$ такой, что для некоторых $i_{1}$ и $i_{2}$ име- 
ем $p_{i_{1}}>0, p_{i_{2}}>0$ (такой вектор $\mathbf{p}$ называется весом). Пусть $\varphi: \mathbb{C}^{n}(\mathbf{p}) \rightarrow \mathbb{C}^{n}-$ раздутие с весом $\mathbf{p}$. Собственный прообраз $X$ на многообразии $\mathbb{C}^{n}(\mathbf{p})$ обозначим через $X(\mathbf{p})$. Раздутие $\varphi: \mathbb{C}^{n}(\mathbf{p}) \rightarrow \mathbb{C}^{n}$ и его ограничение $\left.\varphi\right|_{X(\mathbf{p})}: X(\mathbf{p}) \rightarrow X$

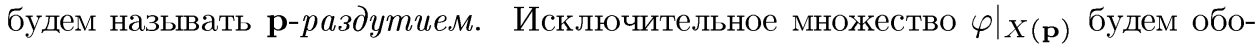
значать через $E_{\mathbf{p}}$. Исключительное множество $D_{\mathbf{p}}$ морфизма $\varphi-$ это взвешенное проективное пространство $\mathbb{P}(\mathbf{p})$. Эти $\mathbf{p}$-раздутия получаются разбиением конуса $\sigma$. Соответствуюший веер состоит из граней конусов $\sigma_{i}$, где $\sigma_{i}$ порождаются векторами $\mathbf{e}_{1}, \ldots, \mathbf{e}_{i-1}, \mathbf{p}, \mathbf{e}_{i+1}, \ldots, \mathbf{e}_{n}$. Векторы $\mathbf{e}_{1}, \ldots, \mathbf{e}_{n}$ - это векторы $(1,0, \ldots, 0), \ldots,(0,0, \ldots, 1)$ соответственно.

Моном $x_{1}^{m_{1}} \ldots x_{n}^{m_{n}} \in \mathbb{C}\left[\left[x_{1}, x_{2}, \ldots, x_{n}\right]\right]$ обозначается через $x^{m}$, где $m=$ $\left(m_{1}, \ldots, m_{n}\right) \in \mathbb{Z}^{n}=M$. Для ряда $f=\sum_{m} a_{m} x^{m} \in \mathbb{C}\left[\left[x_{1}, x_{2}, \ldots, x_{n}\right]\right]$ выполнено $x^{m} \in f$, если $a_{m} \neq 0$. Для $\mathbf{p} \in N_{\mathbb{R}}$ и ряда $f$ определим

$$
\mathbf{p}(f)=\min _{x^{m} \in f}\langle\mathbf{p}, m\rangle .
$$

Главную часть $\sum_{\langle\mathbf{p}, m\rangle=\mathbf{p}(f)} a_{m} x^{m}$ ряда $f$ для $\mathbf{p}$ обозначим через $f_{\mathbf{p}}$.

ОПРЕДЕЛЕНИЕ 1.22. Для ряда $f=\sum_{m} a_{m} x^{m} \in \mathbb{C}\left[\left[x_{1}, x_{2}, \ldots, x_{n}\right]\right]$ определим многогранник Ньютона $\Gamma_{+}(f)$ в $M_{\mathbb{R}}$ следуюшим образом:

$$
\Gamma_{+}(f)=\text { Convex } \bigcup_{x^{m} \in f}\left(m+\sigma^{\vee}\right)
$$

где Convex обозначает выпуклую оболочку. Множество внутренних точек $\Gamma_{+}(f)$ обозначается через $\Gamma_{+}(f)^{0}$. Для любой грани $\gamma$ многогранника $\Gamma_{+}(f)$ определим

$$
f_{\gamma}=\sum_{m \in \gamma} a_{m} x^{m}
$$

Особенность, заданная $f$, называется невырожденной, если для каждой компактной грани $\gamma$ многогранника Ньютона полином $f_{\gamma}=0$ неособ вне множества $x_{1} x_{2} \ldots x_{n}=0$. В этом случае $f$ называется невырожсденным рядом.

ТЕОРема 1.23 [13]. Пусть $(X, 0) \subset\left(\mathbb{C}^{n}, 0\right)$ - нормальная гиперповерхностная особенность, определенная многочленом $f$. Если она каноническая или логканоническая, то $\mathbf{1}=(1, \ldots, 1) \in \Gamma_{+}(f)^{0}$ или $\mathbf{1} \in \Gamma_{+}(f)$ соответственно. Обратное утверждение верно для невырожденных особенностей.

Поясним, как будут изучаться неизолированные гиперповерхностные особенности.

ОПРЕДЕЛЕНИЕ 1.24. Пусть $(X, 0) \subset\left(\mathbb{C}^{n}, 0\right)$ - неизолированная гиперповерхностная особенность (возможно, ненормальная), определенная многочленом $f$. По предложению 1.18 исследовать на исключительность эту особенность можно в произвольно малой окрестности нуля $\mathscr{U}$. Например, для трехмерных логканонических особенностей это означает, что $(\operatorname{Sing} X \backslash \mathbf{0}) \cap \mathscr{U} \cong \bigsqcup_{i} \mathbb{C}_{i}^{1}$ - не линейно связное объединение и вдоль каждой компоненты $X \cap \mathscr{U}$ изоморфно $\mathbb{C}^{1} \times Y$, где $Y-$ двумерная логканоническая гиперповерхностная особенность. В $n$-мерном случае 
пусть $(\operatorname{Sing} X \backslash \mathbf{0}) \cap \mathscr{U} \cong \bigcup_{i=1}^{m}\left(\mathscr{A}_{i}^{n_{i}} \backslash \mathbf{0}\right)$, где $\mathscr{A}_{i}^{n_{i}}$ - неприводимые афффинные многообразия размерности $n_{i}$. Определим попарно различные аффинные многообразия $\left\{V_{j}^{n_{j}}\right\}$, где $n_{j}=\operatorname{dim} V_{j}^{n_{j}}$. Набор индексов $I_{j} \subset\{1, \ldots, m\}$ называется допустимылм, если

$$
\varnothing \neq \bigcap_{i \in I_{j}}\left(\mathscr{A}_{i}^{n_{i}} \backslash \mathbf{0}\right) \nsubseteq \mathscr{A}_{k}^{n_{k}}, \quad k \notin I_{j} .
$$

Для всех различных допустимых наборов $I_{j}$ определим $V_{j}^{n_{j}} \stackrel{\text { def }}{=} \bigcap_{i \in I_{j}} \mathscr{A}_{i}^{n_{i}}$. Пусть $\mathfrak{a}_{j}$ - радикал идеала многообразия $V_{j}^{n_{j}}$ и $f_{1}, \ldots, f_{r}$ - образующие $\mathfrak{a}_{j}$. Тогда имеет место разложение

$$
f=\Phi_{1}\left(f_{1}, \ldots, f_{r}\right) u_{1}\left(x_{1}, \ldots, x_{n}\right)+\cdots+\Phi_{m}\left(f_{1}, \ldots, f_{r}\right) u_{m}\left(x_{1}, \ldots, x_{n}\right),
$$

где $u_{i} \notin \mathfrak{a}_{j}$ для всех $i$. По построению $V_{j}^{n_{j}}$ многообразие $\left\{u_{1}=\cdots=u_{m}=0\right\}$ пусто или неособо. Обозначим $W_{k}=V_{j} \cap\left\{u_{k}=0\right\}$ и повторим для неприводимых компонент непустых множеств $W_{k}$ ту же процедуру, что и для $\mathscr{A}_{i}^{n_{i}}$. В итоге получим множества $W_{k, j}^{n_{k, j}} \subset V_{j}^{n_{j}}$, где $n_{k}-$ их размерность. Для всех $j$ имеем отображение $\psi: X \cap \mathscr{U} \rightarrow V_{j}^{n_{j}}$. По построению для всех точек $P_{1}, P_{2} \in\left(V_{j}^{n_{j}} \backslash\left(\bigcup_{k} W_{k, j}^{n_{k, j}} \cup\right.\right.$ $\mathbf{0}) \backslash\left(\bigcup_{i \neq j} V_{i}^{n_{i}}\right)$ гиперповерхностные особенности размерности $n-1-n_{j}$, соответствующие $\psi^{-1}\left(P_{1}\right)$ и $\psi^{-1}\left(P_{2}\right)$, будут иметь один и тот же тип (разрешение) относительно диаграммы Ньютона, хотя не обязательно будут биголоморфны друг другу. Этот тип обозначим через $\mathscr{F}$. Аналогичное утверждение верно для отображения $\psi: X \cap \mathscr{U} \rightarrow W_{k, j}^{n_{k, j}}$ и множества $\left(W_{k, j}^{n_{k, j}} \backslash \mathbf{0}\right) \backslash \bigcup_{l \neq k} W_{l, j}^{n_{l, j}}$. Соответствуюший тип обозначим через $\mathscr{F}_{j, k}$.

ПримеРы 1.25. 1) Рассмотрим особенность $\left(x_{1}^{2}+x_{2}^{4}+\left(x_{3}^{2}+x_{4}^{3}\right)^{2} x_{4}=0,0\right) \subset$ $\left(\mathbb{C}^{5}, 0\right)$. Тогда $V_{1}^{2}=\left\{x_{1}=x_{2}=x_{3}^{2}+x_{4}^{3}=0\right\}, W_{1,1}^{1}=V_{1}^{2} \cap\left\{x_{4}=0\right\}$, $\mathscr{F}_{1}=\left\{\left(y_{1}^{2}+y_{2}^{4}+y_{3}^{2}=0,0\right) \subset\left(\mathbb{C}^{3}, 0\right)\right\}, \mathscr{F}_{1,1}=\left\{\left(y_{1}^{2}+y_{2}^{4}+y_{3}^{2} y_{4}=0,0\right) \subset\left(\mathbb{C}^{4}, 0\right)\right\}$.

2) Рассмотрим особенность $\left(x_{1}^{2}+x_{2}^{3}+\left(x_{3}^{5}+x_{4}^{5}\right) x_{5}^{2}+x_{3}^{3} x_{4}^{3} x_{5}=0,0\right) \subset\left(\mathbb{C}^{5}, 0\right)$. Тогда $V_{1}^{1}=\left\{x_{1}=x_{2}=x_{3}=x_{4}=0\right\}, V_{2}^{1}=\left\{x_{1}=x_{2}=x_{3}=x_{5}=0\right\}$, $V_{3}^{1}=\left\{x_{1}=x_{2}=x_{4}=x_{5}=0\right\}, \mathscr{F}_{1}=\left\{\left(y_{1}^{2}+y_{2}^{3}+y_{3}^{5}+y_{4}^{5}+c y_{3}^{3} y_{4}^{3}=0,0\right) \subset\left(\mathbb{C}^{4}, 0\right)\right\}$ и для различных значений $c$ особенности могут быть не изоморфными, $\mathscr{F}_{2}=\mathscr{F}_{3}=$ $\left\{\left(y_{1}^{2}+y_{2}^{3}+y_{4}^{2}+y_{3}^{3} y_{4}=0,0\right) \subset\left(\mathbb{C}^{4}, 0\right)\right\}$.

ПРЕДЛОЖЕНИЕ 1.26. Пусть $(X, 0) \subset\left(\mathbb{C}^{n}, 0\right)$ - нормальная неизолированная гиперповерхностная особенность. Она является терминальной вне $\mathbf{0}$, канонической вне $\mathbf{0}$, логканонической вне $\mathbf{0}$ тогда и только тогда, когда для всех $j, k$ особенности $\mathscr{F}_{j} u \mathscr{F}_{j, k}$ соответственно терминальные, канонические, логканонические.

ДокАЗАТЕльство. Докажем предложение, например, для терминального случая. В определении 1.24 мы разбили Sing $X \backslash \mathbf{0}$ на “клетки”, каждая из которых имеет размерность не менее 1 . Вдоль каждой клетки $X$ имеет один и тот же тип особенности. Если некоторая $\mathscr{F}_{j}$ или $\mathscr{F}_{j, k}-$ не терминальная особенность, то при некотором раздутии с центром в соответствующей клетке получим исключительньй дивизор с дискрепантностью не более нуля. Пусть $\mathscr{F}_{j}$ и $\mathscr{F}_{j, k}-$ терминальные особенности, а $(X, 0)$ - не терминальная вне $\mathbf{0}$ особенность. Тогда при логразрешении $\psi$ существует исключительный дивизор $E$ с дискрепантностью $a(E, 0) \leqslant 0$ 
такой, что $\operatorname{dim} \psi(E) \geqslant 1$. Общая точка $P$ из $\psi(E)$ лежит в фиксированной клетке или $P \notin \operatorname{Sing} X$. В обоих случаях имеем противоречие с тем, что $a(E, 0) \leqslant 0$.

ОпРЕДЕЛЕНИЕ 1.27. Ненормальная гиперповерхностная особенность $(X, 0) \subset$ $\left(\mathbb{C}^{n}, 0\right)$ называется логканонической, если пара $\left(\mathbb{C}^{n}, X\right)$ логканонична. Эта же особенность называется логканонической вне $\mathbf{0}$, если пара $\left(\mathbb{C}^{n}, X\right)$ логканонична вне $\mathbf{0}$. Последнее равносильно тому, что $\mathscr{F}_{j}$ и $\mathscr{F}_{j, k}$ имеют логканонические особенности.

\section{§ 2. Квазиоднородные гиперповерхностные особенности}

ОПРЕДЕЛЕНИЕ 2.1. Пусть $f:\left(\mathbb{C}^{n}, 0\right) \rightarrow(\mathbb{C}, 0)-$ многочлен. Он назьвается квазиоднородным степени $d$, если существуют строго положительные рациональные числа $p_{1}, \ldots, p_{n}$, называемые весами, такие, что для любого $\lambda$ и всех $x_{1}, \ldots, x_{n}$ выполнено равенство

$$
f\left(\lambda^{p_{1}} x_{1}, \ldots, \lambda^{p_{n}} x_{n}\right)=\lambda^{d} f\left(x_{1}, \ldots, x_{n}\right) .
$$

Для квазиоднородных особенностей неотрицательные рациональные числа $p_{1}, \ldots$ $\ldots, p_{n}$, удовлетворяющие условию (1), также будут называться весами, a $d \neq 0$ степенью.

В этом параграфе будем считать (не ограничивая обшности), что $p_{1}, \ldots, p_{n}, d-$ целые числа.

Лемма 2.2. Пусть $(X, 0) \subset\left(\mathbb{C}^{n}, 0\right)-$ квазиоднородная гиперповерхностная особенность, определенная многочленом $f$. Если $\frac{\partial f}{\partial x_{1}}(\mathbf{x})=\cdots=\frac{\partial f}{\partial x_{n}}(\mathbf{x})=0$, $\operatorname{mo} f(\mathbf{x})=0$.

ДоКАЗАТЕЛЬСТВо. Многочлен $f$ является квазиоднородным тогда и только тогда, когда $f \in I_{\nabla f}$, где $I_{\nabla f}=\left(\frac{\partial f}{\partial x_{1}}, \ldots, \frac{\partial f}{\partial x_{n}}\right)$ - градиентный идеал [12]. Отсюда следует утверждение леммы.

ПРЕДЛОЖЕНИЕ 2.3. Пусть $(X, 0) \subset\left(\mathbb{C}^{n}, 0\right)$ - нормальная квазиоднородная гиперповерхностная особенность с весами $p_{1}, \ldots, p_{n}$ и степенью $d$, определенная многочленом $f$. Тогда:

1) если $\sum p_{i} \geqslant d+1$ и особенность каноническая вне $\mathbf{0}$, mо $\mathbf{1} \in \Gamma_{+}(f)^{0}$;

2) если $\sum p_{i} \geqslant d$ и особенность логканоническая вне $\mathbf{0}$, то $\mathbf{1} \in \Gamma_{+}(f)$.

ДокаЗАТЕЛЬСТво. Пусть это не так, т.е. $\mathbf{1} \notin \Gamma_{+}(f)^{0}$ или $\mathbf{1} \notin \Gamma_{+}(f)$ соответственно. Тогда существует примитивный вектор $\mathbf{p}^{\prime}$ из $\sigma^{\vee}$ такой, что плоскость $\Pi=\left\{l \in N_{\mathbb{R}} \mid\left\langle\mathbf{p}^{\prime}, l\right\rangle=\mathbf{p}^{\prime}(f)\right\}$ является опорной для многогранника Ньютона, и эта плоскость содержит $\mathbf{1}$ или лежит над 1. Пусть $\varphi: \mathbb{C}^{n}\left(\mathbf{p}^{\prime}\right) \rightarrow \mathbb{C}^{n}-$ $\mathbf{p}^{\prime}$-раздутие. Так как опорная плоскость П параллельна хотя бы одной координатной оси, то по крайней мере одна из координат вектора $\mathbf{p}^{\prime}$ равна нулю. Поэтому $\operatorname{dim} \varphi\left(D_{\mathbf{p}^{\prime}}\right) \geqslant 1$. Рассматривая дискрепантность в первом случае, получаем, что $a\left(D_{\mathbf{p}^{\prime}}, X\right)=\left\langle\mathbf{p}^{\prime}, 1\right\rangle-\mathbf{p}^{\prime}(f)-1 \leqslant-1$, а во втором случае она строго меньше -1 . Возникает противоречие соответственно с каноничностью вне $\mathbf{0}$ и логканоничностью вне $\mathbf{0 .}$

Аналогично доказывается такое же утверждение для ненормальной особенности. 
ПрЕДЛОЖЕНИЕ 2.4. Пусть $(X, 0) \subset\left(\mathbb{C}^{n}, 0\right)$ - ненормальная квазиоднородная гиперповерхностная особенность с весами $p_{1}, \ldots, p_{n}$ и степенью $d$, определенная многочленом $f$. Пусть $\sum p_{i} \geqslant d$ и особенность логканоническая вне $\mathbf{0}$ (см. определение 1.27); тогда $\mathbf{1} \in \Gamma_{+}(f)$.

ОПРЕДЕЛЕНИЕ 2.5. Для каждой гиперповерхностной особенности $(X, 0) \subset$ $\left(\mathbb{C}^{n}, 0\right)$, определенной многочленом $f$, существует веер $\Delta$ (построение веера см., например, в [25]), зависяший только от многогранника Ньютона $\Gamma_{+}(f)$ со следующими свойствами: многообразие $T_{N}(\Delta)$ - гладкое, и поэтому $\operatorname{Diff}_{X(\Delta)}(0)=0$, где $X(\Delta)$ обозначает собственный прообраз $X$ на $T_{N}(\Delta)$. Если $(X, 0)$ - невырожденная особенность, то бирациональный морфизм $\psi: T_{N}(\Delta) \rightarrow \mathbb{C}^{n}$ является торическим логразрешением пары $\left(\mathbb{C}^{n}, X\right)[25]$. Важность невырожденных особенностей состоит в существовании такого торического логразрешения. В любом случае назовем $\psi$ частичным логразрешением и $K_{T_{N}(\Delta)}+X(\Delta)+E(\Delta)=\psi^{*}\left(K_{\mathbb{C}^{n}}+X\right)$.

Теперь предположим, что $f\left(x_{1}, \ldots, x_{n}\right)$ является квазиоднородным многочленом. Выберем такие его веса $\mathbf{p}=\left(p_{1}, \ldots, p_{n}\right)$, что $p_{i} \neq 0$ для всех $i$.

ПримеР 2.6. Если $(X, 0)$ - изолированная особенность, то $\psi$ является ее вложенньм торическим разрешением.

Рассмотрим два случая.

1. Пусть $(X, 0)$ - нормальная особенность, что эквивалентно требованию $\operatorname{codim}_{\mathbb{C}^{n}} \operatorname{Sing} X \geqslant 3$, т.е. $n_{i} \leqslant n-3$ для всех $i$ (см. определение 1.24$)$. Тогда многообразие $X(\Delta)$ нормально и $K_{X(\Delta)}=\psi^{*} K_{X}+\sum a\left(E_{i}, 0\right) E_{i}$. Как и в примеpe 2.6, многообразие $X(\Delta)$ не может иметь изолированные особенности, поэтому $\operatorname{Sing} X(\Delta)=\bigcup_{i \in I} \tilde{\mathscr{A}}_{i}^{n_{i}}$, где $\tilde{\mathscr{A}}_{i}^{n_{i}}$ - неприводимые компоненты и $\psi\left(\tilde{\mathscr{A}}_{i}^{n_{i}}\right)=\mathscr{A}_{i}^{n_{i}}$ (см. определение 1.24).

ОПРЕДЕЛЕНИЕ 2.7. Назовем $\mathscr{A}_{i}^{n_{i}}$ невырожденной компонентой особенности, если $i \notin I$; в противном случае $\mathscr{A}_{i}^{n_{i}}$ назовем вырожденной компонентой особенности. Распознать эти компоненты можно, например, следующим способом. Слегка изменим коэффициенты при мономах из $f$, чтобы особенность стала невырожденной. Если при этом компонента особенности сохранится, то она будет являться невырожденной, если не сохранится, то она - вырождена.

Другими словами, морфизм $\psi$ является логразрешением в точности для невырожденных компонент особенности. Определим $\widetilde{V}_{j}^{n_{j}}, \widetilde{W}_{k, j}^{n_{k, j}}$ так же, как и $V_{j}^{n_{j}}$, $W_{k, j}^{n_{k, j}}$ в определении 1.24. Заметим, что $\psi\left(\widetilde{V}_{j}^{n_{j}}\right)=V_{j}^{n_{j}}, \psi\left(\widetilde{W}_{k, j}^{n_{k, j}}\right)=W_{k, j}^{n_{k, j}}$ и $\widetilde{V}_{j}^{n_{j}}, \widetilde{W}_{k, j}^{n_{k, j}} \nsubseteq \operatorname{Exc}(\psi)$ для всех $j, k$.

ЛЕмма 2.8. Как и в определении 1.24, вдоль множеств

$$
\widetilde{V}_{j}^{n_{j}} \backslash\left(\left(\bigcup_{k} \widetilde{W}_{k, j}^{n_{k, j}}\right) \bigcup\left(\bigcup_{i \neq j} \widetilde{V}_{i}^{n_{i}}\right)\right), \quad \widetilde{W}_{k, j}^{n_{k, j}} \backslash \bigcup_{l \neq k} \widetilde{W}_{l, j}^{n_{l, j}}
$$

особенности имеют тип $\mathscr{F}_{j}, \mathscr{F}_{k, j}$ соответственно. 
ДОКАЗАТЕЛЬСТВО. Исключительный дивизор, соответствуюший р-раздутию, обозначим через $E_{\mathbf{p}}$. Необходимо показать, что тип гиперповерхностных особенностей в точке пересечения этих множеств с $E_{\mathbf{p}}$ будет тот же, т.е. особенность, заданная многочленом

$$
\hat{f}=x_{i}^{-d} f\left(x_{1} x_{i}^{p_{1}}, \ldots, x_{i}^{p_{i}}, \ldots, x_{n} x_{i}^{p_{n}}\right)=f\left(x_{1}, \ldots, 1, \ldots, x_{n}\right)
$$

в окрестности $E_{\mathbf{p}}$, совпадет с особенностью, заданной $f$ в окрестности $x_{i}=1$. Для этого достаточно доказать, что $\frac{\partial f}{\partial x_{i}}(\mathbf{x})=0$, где $\mathbf{x} \in S=\operatorname{Sing}\{\hat{f}=0\}$. Поскольку

$$
\begin{aligned}
\frac{\partial\left(x_{i}^{d} \hat{f}\right)}{\partial x_{i}} & =\sum_{j \neq i} p_{j} \frac{\partial f}{\partial x_{j}}\left(x_{1} x_{i}^{p_{1}}, \ldots\right) x_{j} x_{i}^{p_{j}-1}+p_{i} \frac{\partial f}{\partial x_{i}}\left(x_{1} x_{i}^{p_{1}}, \ldots\right) x_{i}^{p_{i}-1} \\
& =\frac{\partial\left(x_{i}^{d} f\left(x_{1}, \ldots, 1, \ldots, x_{n}\right)\right)}{\partial x_{i}}=d x_{i}^{d-1} f\left(x_{1}, \ldots, 1, \ldots, x_{n}\right)
\end{aligned}
$$

то, подставляя произвольный вектор $\mathbf{x}=\left(x_{1}^{0}, \ldots, 1, \ldots, x_{n}^{0}\right) \in S$, получаем $p_{i} \frac{\partial f}{\partial x_{i}}\left(x_{1}^{0}, \ldots, 1, \ldots, x_{n}^{0}\right)=0$, что и требовалось доказать.

В силу доказательства $\bigcup \operatorname{Sing} E_{i} \subset \operatorname{Sing} X(\Delta)$. Поэтому сушествует логразрешение $\varphi: \widetilde{X} \rightarrow X(\Delta)$ такое, что $\operatorname{dim} \varphi(E) \geqslant 1$, где $E$ - произвольная компонента из $\operatorname{Exc}(\varphi)$, и центр каждого исключительного дивизора не лежит в $\bigcup \operatorname{Sing} E_{i}$. Такое разрешение назовем правильнылм.

2. Пусть $(X, 0)$ - ненормальная приведенная особенность, т.е. $\operatorname{codim}_{\mathbb{C} n} \operatorname{Sing} X \geqslant$ $2\left(n_{i} \leqslant n-2\right.$ для всех $\left.i\right)$. Тогда имеем такое же определение вырожденны $x$ и невырожденных компонент особенности. Аналогично строится правильное логразрешение $\varphi$ пары $\left(T_{N}(\Delta), X(\Delta)+E(\Delta)\right)$ с тем же свойством: центр каждого исключительного дивизора не лежит в $\operatorname{Sing} E(\Delta)$.

Tеорема 2.9. Пусть $(X, 0) \subset\left(\mathbb{C}^{n}, 0\right)$ - нормальная квазиоднородная гиперповерхностная особенность с весами $p_{1}, \ldots, p_{n}$ и степенью $d$, определенная многочленом $f$. Тогда:

1) $\sum p_{i} \geqslant d+1$ и особенность каноническая вне $\mathbf{0}$ тогда и только тогда, когда $(X, 0)$ - каноническая особенность;

2) $\sum p_{i} \geqslant d$ и особенность логканоническая вне 0 тогда и только тогда, когда $(X, 0)$ - логканоническая особенность.

ДокАЗАтЕльство. Достаточность условий очевидна. Докажем необходимость. Пусть $\psi: T_{N}(\Delta) \rightarrow \mathbb{C}^{n}$ - частичное логразрешение (см. определение 2.5 ). Предположим, что выполнено условие 1$)$ теоремы. Тогда по предложению 2.3 $\mathbf{1} \in \Gamma_{+}(f)^{0}$, отсюда $a_{i}\left(E_{i}, 0\right) \geqslant 0$ для всех $i[13]$. Так как $(X, 0)-$ каноническая особенность вне $\mathbf{0}$, то по предложению $1.26 \mathscr{F}_{i}, \mathscr{F}_{k, j}$ - канонические особенности. Отсюда следует, что многообразие $X(\Delta)$ имеет канонические особенности, следовательно, $(X, 0)$ - каноническая особенность. Предположим, что выполнено условие 2$)$ теоремы. Аналогично имеем: $a\left(E_{i}, 0\right) \geqslant-1, \mathscr{F}_{i}, \mathscr{F}_{k, j}-$ логканонические особенности и $X(\Delta)$ - логканоническое многообразие. Так как $\widetilde{V}_{j}^{n_{j}}, \widetilde{W}_{k, j}^{n_{k, j}} \nsubseteq \subseteq \operatorname{Exc}(\psi)$ для всех $j, k$, то, рассматривая правильное логразрешение, получаем, что $(X, 0)$ - логканоническая особенность. 
ЗАмЕчАниЕ 2.10. Согласно теореме 2.9 и предложению 1.26 вопрос о виде конкретной квазиоднородной особенности сводится к исследованию особенностей $\mathscr{F} i$ и $\mathscr{F}_{i, k}$ меньшей размерности.

Аналогично доказывается такой же критерий логканоничности для ненормальной особенности.

Tеорема 2.11. Пусть $(X, 0) \subset\left(\mathbb{C}^{n}, 0\right)$ - ненормальная квазиоднородная гиперповерхностная особенность с весами $p_{1}, \ldots, p_{n}$ u степенью $d$. Тогда $\sum p_{i} \geqslant d$ и особенность логканоническая вне $\mathbf{0}$ тогда и только тогда, когда $(X, 0)$ - логканоническая особенность.

ПримеР 2.12. Рассмотрим особенности из примера 1.25. По теореме 2.9 первая особенность будет канонической, а вторая будет терминальной, так как для нее гиперплоское сечение $\left\{x_{3}=x_{5}\right\}$ имеет канонические особенности и она терминальна вне $\mathbf{0}$.

Tеорема 2.13. Пусть $(X, 0) \subset\left(\mathbb{C}^{n}, 0\right)$ - нормальная логканоническая квазиоднородная гиперповерхностная особенность с весами $\mathbf{p}=\left(p_{1}, \ldots, p_{n}\right)$, определенная многочленом $f$. Тогда:

1) если $(X, 0)$ - не каноническая вне $\mathbf{0}$, то р-раздутие является логканоническим, но не чисто логтерминальным раздутием, за исключением случая, описанного в примере $2.15,1)$; в частности, в обоих случаях $(X, 0)$ - неисключительная особенность;

2) если $(X, 0)$ - каноническая вне $\mathbf{0}$, то р-раздутие является чисто логтерминальныцм раздутием, если дополнительно предположить, что $(X, 0)$ имеет строго логканонические особенности, то $(X, 0)$ - исключительная особенность (ср. [19, предложение 3.3$])$.

ДокаЗАТЕльство. Пусть $\psi: \mathbb{C}^{n}(\mathbf{p}) \rightarrow \mathbb{C}^{n}-\mathbf{p}$-раздутие. Тогда исключительное множество имеет вид

$$
\operatorname{Exc}\left(\left.\psi\right|_{X(\mathbf{p})}\right)=E_{\mathbf{p}}=D_{\mathbf{p}} \cap X(\mathbf{p})=\left\{f\left(x_{1}, \ldots, x_{n}\right) \subset \mathbb{P}\left(p_{1}, \ldots, p_{n}\right)\right\} .
$$

Так как $f$ - неприводимый многочлен, то $E_{\mathbf{p}}$ - неприводимое многообразие. Очевидно, что $-E_{\mathbf{p}}$ относительно обилен и $X(\mathbf{p})$ - нормальное многообразие. Рассмотрим частичное разрешение $X$, ему соответствует морфизм $\varphi: T_{N}(\Delta) \rightarrow \mathbb{C}^{n}(\mathbf{p})$. Имеем

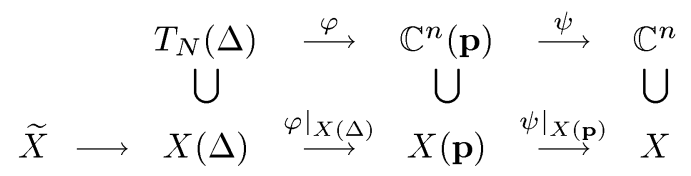

Тогда

$$
K_{T_{N}(\Delta)}+X(\Delta)+D_{\mathbf{p}}=\varphi^{*}\left(K_{\mathbb{C}^{n}(\mathbf{p})}+X(\mathbf{p})+D_{\mathbf{p}}\right)+\sum_{\mathbb{R}_{\geqslant 0} \mathbf{q} \in \Delta(1), \mathbf{q} \neq \mathbf{p}} \alpha_{\mathbf{q}} D_{\mathbf{q}}
$$

Дивизоры, соответствуюшие вектору $\mathbf{p}$ на многообразиях $T_{N}(\Delta)$ и $\mathbb{C}^{n}(\mathbf{p})$, обозначим одинаково через $D_{\mathbf{p}}$. Пусть примитивный вектор $\mathbf{q} \in \sigma_{i}$. Тогда из доказательства леммы 3.2 из [19] имеем

$$
\alpha_{\mathbf{q}}=a\left(D_{\mathbf{q}}, X(\mathbf{p})\right)-\frac{q_{i}}{p_{i}}=\langle\mathbf{q}, 1\rangle-\mathbf{q}(f)-\frac{q_{i}}{p_{i}}(\langle\mathbf{p}, 1\rangle-\mathbf{p}(f))-1 .
$$


Предположим, что выполнено условие 1) теоремы. Тогда $1 \in \Gamma_{+}(f)$. Если $q_{i}=0$, то $\alpha_{\mathbf{q}}=\langle\mathbf{q}, 1\rangle-\mathbf{q}(f)-1 \geqslant-1$. Если $q_{i}>0$, то определим вектор $\mathbf{a}=\left(a_{1}, \ldots, a_{n}\right)$ из пропорции $\left(q_{1}: \ldots: q_{n}\right)=\left(p_{1}+a_{1}: \ldots: p_{n}+a_{n}\right)$. Тогда $a_{i}=0$ и $a_{j} \geqslant 0$ для всех $j \neq i$. Подставим $\mathbf{q}=\frac{q_{i}}{p_{i}}(\mathbf{p}+\mathbf{a})$. Тогда $\alpha_{\mathbf{q}}=\frac{q_{i}}{p_{i}}(\langle\mathbf{a}, 1\rangle-$ $\mathbf{a}(f))-1 \geqslant-1$.

ЛЕмма 2.14. $\operatorname{Diff}_{X(\mathbf{p})}(0)=0$.

ДокАЗАТЕльство. Если $\operatorname{codim}_{\mathbb{C}^{n}(\mathbf{p})} \operatorname{Sing} \mathbb{C}^{n}(\mathbf{p})>2$, то лемма доказана. Пусть $\operatorname{codim}_{\mathbb{C}^{n}(\mathbf{p})} \operatorname{Sing} \mathbb{C}^{n}(\mathbf{p})=2$. Тогда возможны два случая.

1) $\left(p_{1}, \ldots, p_{j-1}, p_{j+1}, \ldots, p_{n}\right)=d>1$ и $p_{j} \neq 0$. Отсюда $\psi\left(D_{\mathbf{p}}\right)=0$.

Рассмотрим произвольную карту на многообразии $\mathbb{C}^{n}(\mathbf{p})$

$$
U_{k}=\mathbb{C}_{y_{1}, \ldots, y_{n}}^{n} / \mathbb{Z}_{p_{k}}\left(-p_{1}, \ldots,-p_{k-1}, 1,-p_{k+1}, \ldots,-p_{n}\right), \quad k \neq j
$$

Многообразие $X(\mathbf{p})$ задается в этой карте уравнением $f\left(y_{1}, \ldots, y_{k-1}, 1, y_{k+1}, \ldots\right.$ $\left.\ldots, y_{n}\right)=0$. Если $\operatorname{Diff}_{X(\mathbf{p})}(0) \neq 0$, то для всех $\mathbf{y}=\left(y_{1}, \ldots, y_{k-1}, 1, y_{k+1}, \ldots, y_{j-1}\right.$, $\left.0, y_{j+1}, \ldots, y_{n}\right)$ имеем $f(\mathbf{y})=0$. Отсюда $f=x_{j} f^{\prime}$, а это невозможно.

2) $p_{i} \neq 0$ для $i \in I$ и $p_{i}=0$ для $i \notin I$, где $I \subset\{1, \ldots, n\}$ и $2 \leqslant k=|I| \leqslant n-1$. Отсюда $\operatorname{dim} \psi\left(D_{\mathbf{p}}\right)=n-k$.

В малой окрестности $\mathscr{U}$ обшей точки $P \in \psi\left(D_{\mathbf{p}}\right)$ многообразие $X$ изоморфно $\mathbb{C}^{n-k} \times X^{\prime}$. Морфизм $\psi$ индуцирует $\mathbf{p}^{\prime}$-раздутия $\mathbb{C}^{k}\left(\mathbf{p}^{\prime}\right) \rightarrow \mathbb{C}^{k}$ и $X^{\prime}\left(\mathbf{p}^{\prime}\right) \rightarrow X^{\prime}$, где $p_{i}^{\prime}=p_{i}$ для $i \in I$. По предыдушему пункту $\operatorname{Diff}_{X^{\prime}\left(\mathbf{p}^{\prime}\right)}(0)=0$ и, следовательно, $\operatorname{Diff}_{X(\mathbf{p})}(0)=0$.

Из этой леммы и равенства (2) имеем

$$
K_{X(\Delta)}+\widetilde{E}_{\mathbf{p}}=\varphi^{*}\left(K_{X(\mathbf{p})}+E_{\mathbf{p}}\right)+\sum \alpha_{\mathbf{q}}^{\prime} D_{\mathbf{q}}^{\prime}, \quad \alpha_{\mathbf{q}}^{\prime} \geqslant-1
$$

При правильном разрешении особенностей $\widetilde{X} \rightarrow X(\Delta)$ все исключительные дивизоры для пары $\left(X(\Delta), \widetilde{E}_{\mathbf{p}}-\sum \alpha_{\mathbf{q}}^{\prime} D_{\mathbf{q}}^{\prime}\right)$ появятся с дискрепантностью не меньше -1 , так как $\widetilde{V}_{j}^{n_{j}}, \widetilde{W}_{k, j}^{n_{k, j}} \nsubseteq \operatorname{Exc}(\psi \circ \varphi)$. Поскольку особенность не каноническая вне $\mathbf{0}$, то либо пара $\left(X(\mathbf{p})+E_{\mathbf{p}}\right)$ не чисто логтерминальна, либо имеем случай, описанный в примере $2.15,1)$. По предложениям 1.17 и 1.18 особенность $(X, 0)$ неисключительна.

Пусть выполнено условие 2) теоремы. Тогда достаточно показать, что при $q_{i}=0$ дискрепантность $\alpha_{\mathbf{q}}=\langle\mathbf{q}, 1\rangle-\mathbf{q}(f)-1$ больше -1 и $\langle\mathbf{a}, 1\rangle-\mathbf{a}(f)>0$ при $q_{i}>0$. Действительно, если $\alpha_{\mathbf{q}}=-1$, то при q- или а-раздутии имеем противоречие с каноничностью вне $\mathbf{0}$. Может так случиться, что а-раздутие не определено (см. определение 1.21). Тогда если $\mathbf{a}(f)=0$, то теорема доказана, а если $f$ - приводимый многочлен, то возникает противоречие с нормальностью особенности.

Предположим дополнительно, что $(X, 0)$ имеет строго логканонические особенности. Тогда $p_{i} \neq 0$ для всех $i$, иначе имеем противоречие с каноничностью вне $\mathbf{0}$, т.е. $\operatorname{dim} \psi\left(E_{\mathbf{p}}\right)=0$. В силу [11, теорема 1.9$]$ особенность $(X, 0)$ исключительна. Теорема полностью доказана. 
ПримеР 2.15. 1) Пусть $(X, 0)$ - нормальная квазиоднородная строго логканоническая неизолированная особенность с весом $\mathbf{p}$. Пусть $\mathbf{p}$-раздутие $\psi$ является чисто логтерминальным и $p_{i}=0$ хотя бы для одного $i$. Это означает, что существует ровно один индекс $k$, для которого особенность $\mathscr{F}_{k}$ строго логканоническая. Заметим, что особенность $\mathscr{F}_{k}$ исключительна, соответствующая компонента $\mathscr{A}_{k}^{n_{k}}$ невырождена и $\psi\left(E_{\mathbf{p}}\right)=\mathscr{A}_{k}^{n_{k}}$.

2) Пусть $(X, 0)$ - нормальная строго логканоническая гиперповерхностная особенность, заданная многочленом $f$. Пусть для $\mathbf{p} \in N_{\mathbb{R}}\left(X_{\mathbf{p}}, 0\right)=\left(f_{\mathbf{p}}=0,0\right)$ является особенностью из п. 1$)$. Тогда р-раздутие будет также чисто логтерминальным.

ПримеР 2.16. Рассмотрим особенность $\left(x_{1}^{2}+x_{2}^{3}+x_{3}^{6}=0,0\right) \subset\left(\mathbb{C}^{4}, 0\right)$ с весами $\mathbf{p}=(3,2,1, a)$. Если $a=0$, то $\mathbf{p}$-раздутие является чисто логтерминальным. Если $a \neq 0$, то $\mathbf{p}$-раздутие является логканоническим, но не чисто логтерминальным.

Для ненормальных особенностей можно доказать аналогичную теорему.

Tеорема 2.17. Пусть $(X, 0) \subset\left(\mathbb{C}^{n}, 0\right)$ - ненормальная логканоническая квазиоднородная гиперповерхностная особенность с весами $\mathbf{p}=\left(p_{1}, \ldots, p_{n}\right)$, определенная многочленом $f$. Тогда после $\mathbf{p - р а з д у т и я ~} \psi: \mathbb{C}^{n}(\mathbf{p}) \rightarrow \mathbb{C}^{n}$ дивизор $K_{\mathbb{C}^{n}(\mathbf{p})}+X(\mathbf{p})+D_{\mathbf{p}}$ будет логканоническим, но не чисто логтерминальHbl.M.

Из теоремы 2.13 имеем следуюшее важное следствие.

СлЕДСТвИЕ 2.18. Пусть $(X, 0) \subset\left(\mathbb{C}^{n}, 0\right)$ - нормальная гиперповерхностная особенность, определенная многочленом $f$, и пусть $\mathbf{p} \in N_{\mathbb{R}}$. Положим $\left(X_{\mathbf{p}}, 0\right)=\left(f_{\mathbf{p}}=0,0\right)$. Тогда:

1) если $\left(X_{\mathbf{p}}, 0\right)$ - каноническая (логканоническая) особенность, то $(X, 0)$ также каноническая (логканоническая) особенность;

2) для $\mathbf{p}$-раздутий $\varphi:\left(X(\mathbf{p}), E_{\mathbf{p}}\right) \rightarrow(X, 0)$ u $\varphi_{\mathbf{p}}:\left(X_{\mathbf{p}}(\mathbf{p}), E_{\mathbf{p}}^{\prime}\right) \rightarrow\left(X_{\mathbf{p}}, 0\right)$ имеем $\left(E_{\mathbf{p}}, \operatorname{Diff}_{E_{\mathbf{p}}}(0)\right)=\left(E_{\mathbf{p}}^{\prime}, \operatorname{Diff}_{E_{\mathbf{p}}^{\prime}}(0)\right)$;

3) если $\left(X_{\mathbf{p}}, 0\right)$ - логканоническая особенность, не каноническая вне $\mathbf{0}$, то р-раздутие является логканоническим, но не чисто логтерминальным раздутием $(X, 0)$, кроме случая, описанного в примере $2.15,2)$; в частности, $(X, 0)$ - неисключительная особенность;

4) если $\left(X_{\mathbf{p}}, 0\right)$ - логканоническая особенность, каноническая вне $\mathbf{0}$, то p-раздутие является чисто логтерминальньцм раздутием $(X, 0)$; если дополнительно предположить, ито $(X, 0)$ имеет строго логканонические особенности, то $(X, 0)$ - исключительная особенность.

ДоказАтЕльСтво. Рассмотрим деформацию $F_{t}=t^{-\mathbf{p}(f)} f\left(t^{p_{1}} x_{1}, \ldots, t^{p_{n}} x_{n}\right)$. При $t=0$ вьполнено $F_{0}=f_{\mathbf{p}}$, а при малых $t \neq 0$ деформация $F_{t}$ имеет тип $f$. Пусть $\left(X_{\mathbf{p}}, 0\right)$ - логканоническая особенность; тогда по $[9$, лемма 7.1 .3$]$ пара $\left(\mathbb{C}^{n}, X_{\mathbf{p}}\right)$ логканонична. Отсюда и из обрашения присоединения и $[9$, следствие 7.8$]$ следует первое утверждение. Утверждения 2) и 4) очевидны. Для доказательства утверждения 3$)$ достаточно проверить, что пара $\left(\mathbb{C}^{n}(\mathbf{p}), X(\mathbf{p})+D_{\mathbf{p}}\right)$ логканонична. Рассмотрим ту же деформацию. При $t=0$ из доказательства теоремы 2.13 
пара $\left(\mathbb{C}^{n}(\mathbf{p}), X_{\mathbf{p}}(\mathbf{p})+D_{\mathbf{p}}\right)$ логканонична, поэтому из $[9$, следствие 7.8$]$ получаем требуемое.

СлЕДСТвИЕ 2.19. Пусть $(X, 0) \subset\left(\mathbb{C}^{n}, 0\right)$ - нормальная логканоническая гиперповерхностная особенность, определенная многочленом $f, u\left(f_{\mathbf{p}}=0,0\right)$ ненормальная логканоническая особенность для некоторого $\mathbf{p} \in N_{\mathbb{R}}$. Тогда p-раздутие является логканоническим, но не чисто логтерминальным раздутием $(X, 0)$. В частности, $(X, 0)$ - неисключительная особенность.

ДокаЗАТЕльСтво. Так как $f_{\mathbf{p}}$ - приведенный многочлен, то в каждой карте для р-раздутия $[20$, п. 3.2$]$ легко проверить, что $\operatorname{dim} \operatorname{Sing} X(\mathbf{p}) \leqslant n-2$. Поэтому $X(\mathbf{p})$ будет нормальным многообразием. Дальнейшее рассуждение аналогично доказательству следствия 2.18 (с использованием теоремы 2.17).

ЗАмечАниЕ 2.20. Для невырожденных логканонических (соответственно, канонических) особенностей всегда существует $\mathbf{p} \in N_{\mathbb{R}}$ такой, что $f_{\mathbf{p}}$ задает логканоническую (соответственно, каноническую) особенность [19, теорема 3.5$]$.

\section{§ 3. Трехмерные логканонические гиперповерхностные особенности}

Приведем доказательство основной теоремы, сформулированной во введении.

ДОКАЗАТЕЛЬСТВо ОСНовНоЙ ТЕОРЕМЫ. По теореме 3.2 (см. далее) исключительные строго логканонические (канонические) гиперповерхностные особенности делятся на два типа:

1) сушествует примитивный вектор $\mathbf{p} \in N_{\mathbb{R}}$ такой, что $\tilde{f}_{\mathbf{p}}=(f \circ \psi)_{\mathbf{p}}$ задает строго логканоническую и каноническую вне $\mathbf{0}$ (каноническую) особенность;

2) $\tilde{f}_{\mathbf{p}}=t^{3}+g_{2}^{2}(z, x, y)$, где $g_{2}$ - неприводимый однородный многочлен степени 2.

Исключительные особенности типа 2) классифицированы в теоремах 3.3 и 3.5 . Рассмотрим особенности типа 1). Вектор $\mathbf{p}$ единственный. Если существуют два различных $\mathbf{p}_{1}$ и $\mathbf{p}_{2}$, то согласно $[19$, лемма 4.1] они задают два разных логканонических раздутия (следствие 2.18). Поэтому по предложению 1.17 особенность $(X, 0)$ неисключительна. Итак, согласно следствию $2.18 \mathbf{p}$-раздутие $\varphi:\left(X(\mathbf{p}), E_{\mathbf{p}}\right) \rightarrow(X, 0)$ является чисто логтерминальным.

Пусть $\tilde{f}_{\mathrm{p}}$ определяет строго логканоническую особенность. Тогда по следствию $2.18(X, 0)$ - исключительная особенность. Так как $\operatorname{dim} \varphi\left(E_{\mathbf{p}}\right)=0$ (см. [11, теорема 1.9]) и $\langle\mathbf{p}, 1\rangle=\mathbf{p}(f)$, то $\operatorname{Diff}_{E_{\mathbf{p}} / \mathbb{P}(\mathbf{p})}(0)=0$ и $\operatorname{Diff}_{E_{\mathbf{p}}}(0)=0$. Отсюда $E_{\mathbf{p}}-$ поверхность с дювалевскими особенностями и $K_{E_{\mathbf{p}}} \sim 0$. В силу [2, лемма 1.4.1] $E_{\mathbf{p}}$ - особая К3-поверхность.

Пусть $\tilde{f}_{\mathbf{p}}$ определяет каноническую особенность. Тогда при предположении $\operatorname{Diff}_{E_{\mathbf{p}} / \mathbb{P}(\mathbf{p})}(0)=0$ такие особенности классифицированы в теоремах $3.24,3.29$ и в таблицах $\S 4$. Обоснование этой классификации начинается с теоремы 3.6. Основная теорема доказана.

ОпРЕдЕЛЕниЕ 3.1. Квазиоднородная гиперповерхностная особенность $f$ принадлежит классу $\mathscr{M}_{i}$, если максимальная компактная грань многогранника Ньютона $\Gamma_{+}(f)$ имеет размерность $i$.

Следуюшая теорема разбивает исключительные трехмерные гиперповерхностные особенности на два типа. 
Tеорема 3.2. Пусть $(Z, 0) \subset\left(\mathbb{C}^{4}, 0\right)$ - трехмерная исключительная каноническая (соответственно, строго логканоническая) гиперповерхностная особенность, заданная многочленом $g$. Тогда существуют биголоморфная замена координат $\psi:\left(\mathbb{C}^{4}, 0\right) \rightarrow\left(\mathbb{C}_{t, z, x, y}^{4}, 0\right)$ и примитивныи иектор $\mathbf{p} \in N_{\mathbb{R}}$ такие, что выполняется одно из следующих условий:

1) $\tilde{g}_{\mathbf{p}}=(g \circ \psi)_{\mathbf{p}}$ задает каноническую (соответственно, строго логканоническую и каноническую вне $\mathbf{0}$ ) особенность;

2) $\tilde{g}_{\mathbf{p}}=t^{3}+g_{2}^{2}(z, x, y)$, где $g_{2}-$ неприводимый однородный многочлен степени 2.

ДокАЗАТЕЛЬСтво. Рассмотрим сначала канонические особенности. Если $g-$ квазиоднородный многочлен, то все доказано. Возьмем $\mathbf{p} \in N_{\mathbb{R}}$ такой, что $g_{\mathbf{p}} \in$ $\mathscr{M}_{3}, \mathbf{1} \in \Gamma_{+}\left(g_{\mathbf{p}}\right)^{0}$ и $\mathbf{p}(g)=1$. Очевидно, что 2 - или 3 -струя $g_{\mathbf{p}}$ ненулевая. Поэтому если $g_{\mathbf{p}}-$ неприведенный многочлен, т.е. $g_{\mathbf{p}}=\left(g_{\mathbf{p}}^{\prime}\right)^{k}$, где $k \geqslant 2$, то, не ограничивая обшности, считаем, что $g_{\mathrm{p}}^{\prime}=t+g^{\prime \prime}$. После квазиоднородной замены $\psi: t \longmapsto t-g^{\prime \prime}$ получим $\tilde{g}=g \circ \psi=t^{k}+\tilde{g}^{\prime}$ и $\tilde{g}_{\mathbf{p}}=t^{k}$. Повторим описанную процедуру для $\tilde{g}$. Так как $\mathbf{1} \in \Gamma_{+}(g)^{0}$, то этот процесс оборвется. Итак, будем считать, что $g_{\mathbf{p}}-$ приведенный многочлен.

Пусть $\left(Z_{\mathbf{p}}, 0\right)=\left(\left\{g_{\mathbf{p}}=0\right\}, 0\right)-$ ненормальная особенность. Тогда все компоненты $\mathscr{A}_{i}^{2}$ являются вырожденными (ср. [19, предложение 3.3$\left.]\right)$. Пусть $\mathscr{A}_{j}^{2}$ - невырожденная компонента. Тогда при частичном разрешении $\psi$ сушествует исключительньй дивизор $E$ с дискрепантностью не более -1 такой, что $\psi(E)=\mathscr{A}_{j}^{2}$. Возникает противоречие с условием $1 \in \Gamma_{+}\left(g_{\mathbf{p}}\right)^{0}$. Из следствия 2.19 вдоль некоторой компоненты $\mathscr{A}_{k}^{2}$ имеем ненормальные не логканонические особенности. В окрестности общей точки $\mathscr{A}_{k}^{2}$ имеем

$$
Z_{\mathbf{p}} \cong \mathbb{C}_{f_{1}, f_{2}}^{2} \times\left\{\Phi\left(f_{1}(t, z, x, y), f_{2}(t, z, x, y)\right)=0\right\}, \quad \operatorname{deg} \Phi \geqslant 2 .
$$

Так как $\mathbf{1} \notin \Gamma_{+}(\Phi), 2$ - или 3 -струя $g_{\mathbf{p}}$ ненулевая, то после квазиоднородной биголоморфной замены можно считать, что $f_{1}=t$. Если mult $f_{2}=1$, то несложно показать, что в $f_{2}$ входит моном $x$, либо $y$, либо $z$. После соответствующей квазиоднородной замены координат будем считать, что mult $f_{0} f_{2} \geqslant 2$. Пусть $\Phi_{\mathbf{q}}-$ квазиоднородная часть $\Phi(\mathbf{q}(\Phi)=1)$, которая определяет изолированную особенность. Тогда wt $z+\operatorname{wt} x+$ wt $y \leqslant 3 \cdot \frac{1}{2}$ wt $f_{2}$ и равенство достигается, когда $f_{2}=g_{2}-$ неприводимый однородный многочлен степени 2 . Исходя из этого, решаем систему

$$
\left\{\begin{array}{l}
\text { wt } t+\text { wt } z+\text { wt } x+\text { wt } y>1 \\
\text { wt } f_{1}+\text { wt } f_{2}<1 \\
\text { wt } f_{1}, \text { wt } f_{2} \in\left\{\frac{1}{2}, \frac{1}{3}\right\} \cup\left(0, \frac{1}{3}\right)
\end{array}\right.
$$

и получаем, что $\Phi_{\mathbf{q}}=f_{1}^{3}+f_{2}^{2}$. Отсюда $g_{\mathbf{p}}=t^{3} u_{1}(t, z, x, y)+g_{2}^{2} u_{2}(t, z, x, y)$. Так как $\mathbf{1} \in \Gamma_{+}\left(g_{\mathbf{p}}\right)^{0}$, то $u_{1}, u_{2}-$ константы и имеет место случай 2$)$ теоремы.

Пусть $\left(Z_{\mathbf{p}}, 0\right)$ - нормальная не каноническая особенность. Тогда по теореме 2.9 эта особенность не канонична вне $\mathbf{0}$. Аналогично, вдоль некоторой вырожденной компоненты $\mathscr{A}_{k}^{1}$ имеем не каноническую особенность $\Phi\left(f_{1}, f_{2}, f_{3}\right)=0$. Если 
mult $_{0} f_{i}=1$ для всех $i$, то произведем соответствующую замену и повторим процедуру. Пусть mult $f_{3} \geqslant 2$; тогда, рассматривая квазиоднородную часть $\Phi$, имеем

$$
\text { wt } t+\text { wt } z+\text { wt } x+\text { wt } y \leqslant \text { wt } f_{1}+\text { wt } f_{2}+\frac{1}{2} \text { wt } f_{3}+\frac{1}{2} \text { wt } f_{3} \leqslant 1 .
$$

Противоречие с построением $g_{\mathbf{p}}$.

Рассмотрим строго логканонические особенности. Аналогично выберем $\mathbf{p} \in$ $N_{\mathbb{R}}$ такой, что $g_{\mathbf{p}} \in \mathscr{M}_{3}, \mathbf{1} \in \Gamma_{+}\left(g_{\mathbf{p}}\right)$ и $\mathbf{p}(g)=1$. Более того, если $g_{\mathbf{p}}$ задает не логканоническую особенность вне $\mathbf{0}$, то $\mathbf{1} \in \Gamma_{+}\left(g_{\mathbf{p}}\right)^{0}$. Поэтому применим те же рассуждения. Теорема доказана.

Следующая теорема описьвает все исключительные (слабо исключительные) канонические особенности в случае 2) теоремы 3.2 .

Teоpema 3.3. Пусть $(X, 0)=\left(f=t^{3}+g_{2}^{2}(z, x, y)+f_{\geqslant 5}^{\prime}(z, x, y)=0,0\right) \subset$ $\left(\mathbb{C}^{4}, 0\right)$ - каноническая особенность. Тогда существует чисто логтерминальное раздутие $\varphi:(Y, S) \rightarrow(X \ni 0)$ такое, ито $\left(S, \operatorname{Diff}_{S}(0)\right)=\left(\overline{\mathbb{F}}_{10}, \frac{1}{2} H_{1}+\frac{2}{3} H_{2}\right)$, где $\overline{\mathbb{F}}_{10}=\mathbb{P}(10,1,1)$ - конус, $H_{1}$ и $H_{2}$ - его гиперплоские сечения. Более того, особенность $(X, 0)$ является исключительной (слабо исключительной) $и$ 6-дополняемой тогда и только тогда, когда неприводимые мнохители ненулевой 5-струи $f_{5}^{\prime}$ имеют кратности не более 3 (не более 4).

ДоказАтельство. Рассмотрим $\mathbf{p}$-раздутие $\phi:(X(\mathbf{p}), E) \rightarrow X$, где $\mathbf{p}=(4,3$, $3,3)$. Тогда $\left(E, \operatorname{Diff}_{E}(0)\right)=\left(t+g_{2}^{2} \subset \mathbb{P}(4,1,1,1), \frac{2}{3}\{t=0\}\right)=\left(\mathbb{P}^{2}, \frac{4}{3} C\right)$, где $C$ - неприводимая коника (см. определение 3.12 ). Дискрепантность $a(E, 0)$ равна нулю. Если 5-струя $f^{\prime}$ нулевая, то многочлен $f$ в карте $\mathbb{C}_{t^{\prime}, z^{\prime}, x^{\prime}, y^{\prime}}^{4} / \mathbb{Z}_{3}(2,1,0,0)$ имеет вид $t^{\prime 3}+g_{2}^{2}\left(1, x^{\prime}, y^{\prime}\right)+z^{\prime 6} f^{\prime \prime}$. Эта особенность не каноническая, поэтому 5 -струя $f^{\prime}$ ненулевая. Теперь теорема доказывается перебором всех случаев для 5 -струй.

Пусть $f=t^{3}+g_{2}^{2}+z^{5}+f_{\geqslant 6}^{\prime}(z, x, y)$. Имеем следующую картину:

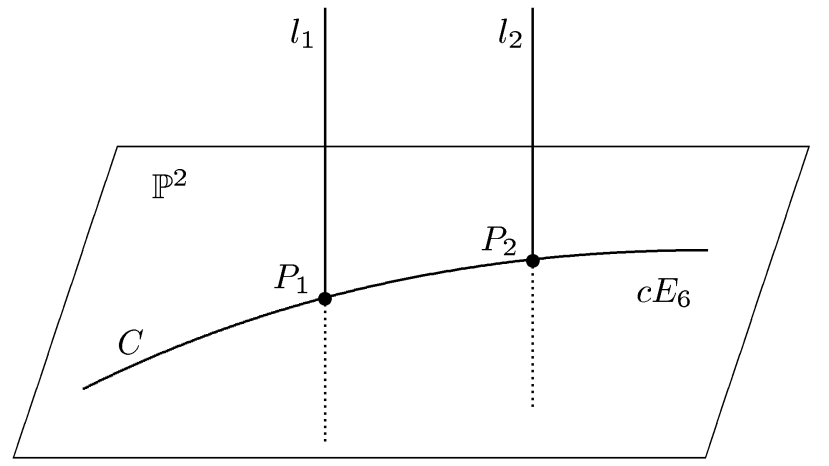

Многообразие $X(\mathbf{p})$ в окрестности точек $P_{i}$ (точки $P_{i}$ имеют координаты $(0$, $\left.1, y_{i}\right)$ в $\mathbb{P}^{2}$, где $\left.g_{2}\left(0,1, y_{i}\right)=0\right)$ имеет вид

$$
\left(t^{\prime 3}+\omega^{2}+z^{\prime 5} x^{\prime 3}+x^{\prime 6} f^{\prime \prime}=0 \subset\left(\mathbb{C}_{t^{\prime}, \omega, z^{\prime}, x^{\prime}}^{4}, 0\right)\right) / \mathbb{Z}_{3}(2,0,0,1) .
$$


В зависимости от вида $f^{\prime}$ эта особенность может быть неизолированной вдоль кривых $l_{1}$ и $l_{2}$, не лежаших на $E$. Подсчитывая инварианты, получаем, что многообразие $X(\mathbf{p})$ в окрестности точки $P_{i}$ изоморфно многообразию

$$
\left(x_{1}^{2} x_{4}^{5}+x_{1} x_{3}^{2}+x_{2}^{3}+f^{\prime \prime \prime}=0,0\right) \subset\left(\mathbb{C}_{x_{1}, x_{2}, x_{3}, x_{4}}^{4}, 0\right) .
$$

Поверхность $E$ задается уравнениями $\left\{x_{1}=x_{3}=0\right\}$. Вдоль кривой $C$ многообразие $X(\mathbf{p})$ имеет особенность $c E_{6}$. Пусть $H$ - общее гиперплоское сечение $X(\mathbf{p})$, проходящее через произвольную точку из $C \backslash\left\{P_{1}, P_{2}\right\}$, и $C^{\prime}=E \cap H$. Тогда на минимальном разрешении $H$ имеем следуюший граф:

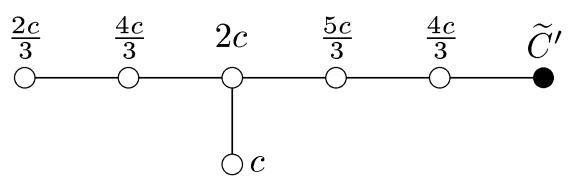

где $\widetilde{C}^{\prime}$ - собственный прообраз $C^{\prime}$. Числа у вершин графа обозначают соответствующие им дискрепантности логпары $\left(H, c C^{\prime}\right)$. Отсюда видно, что логканонический порог этой пары равен $1 / 2$ и раздутие центральной вершины графа будет индуктивным раздутием пары $\left(H, \frac{1}{2} C^{\prime}\right)$ (см. [11, теорема 1.5$\left.]\right)$. Пусть $c=$ $c(X(\mathbf{p}), E)$ - логканонический порог. В окрестностях точек $P_{1}, P_{2}$ пара $(X(\mathbf{p}), E)$ имеет одинаковые особенности, поэтому по теореме о связности [8, теорема 17.4$]$ $\operatorname{LCS}(X(\mathbf{p}), c E)=C$. Согласно доказанному вьше $c=1 / 2$.

Раздуем $\mathbb{C}^{4}(\mathbf{p})$ вдоль кривой $C$ с весами $(6,4,3,0)$. Тогда это раздутие индуцирует $\psi: \widetilde{X}(\mathbf{p}) \rightarrow X(\mathbf{p})$ и имеем

$$
K_{\widetilde{X}(\mathbf{p})}+\widetilde{S}+\frac{1}{2} E_{\widetilde{X}(\mathbf{p})}=\psi^{*}\left(K_{X(\mathbf{p})}+\frac{1}{2} E\right) .
$$

Очевидно, что $f^{\prime \prime \prime}$ не влияет на вклеенную поверхность $\widetilde{S} \cong \mathbb{F}_{10}$. В дальнейшем будет показано, что собственньй прообраз $E_{\widetilde{X}}(\mathbf{p})$ дивизора $E$ можно стянуть в точку. Поэтому он будет пересекаться с $\widetilde{S}$ по минимальному сечению. Имеем

$$
\begin{aligned}
\operatorname{Diff}_{\widetilde{S}}\left(\frac{1}{2} E_{\widetilde{X}(\mathbf{p})}\right) & =\operatorname{Diff}_{\widetilde{S}}(0)+\left.\frac{1}{2} E_{\widetilde{X}(\mathbf{p})}\right|_{\widetilde{S}}=\frac{2}{3} l_{\infty}+\frac{1}{2} l_{0}+\frac{2}{3} l_{0}^{\prime}+\frac{1}{2} \cdot \frac{1}{3} l_{\infty} \\
& =\frac{5}{6} l_{\infty}+\frac{1}{2} l_{0}+\frac{2}{3} l_{0}^{\prime},
\end{aligned}
$$

где $l_{\infty}$ - минимальное сечение, а $l_{0}, l_{0}^{\prime}$ - различные нулевые сечения, которые пересекаются в двух точках с кратностью 5 . Эти точки находятся над точками $P_{1}$ и $P_{2}$. Очевидно, что логповерхность $\left(\widetilde{S}, \operatorname{Diff}_{\widetilde{S}}\left(\frac{1}{2} E_{\widetilde{X}(\mathbf{p})}\right)\right)$ логтерминальна по Кавамате, т.е. $\psi$ - индуктивное раздутие логканонической пары $\left(X(\mathbf{p}), \frac{1}{2} E\right)$. Пусть $L_{X(\mathbf{p})} \in\left|-n\left(K_{X(\mathbf{p})}+\frac{1}{2} E\right)\right|-$ обший элемент из очень обильной над $X$ линейной системы $(n \gg 0)$. Тогда дивизор $K_{X(\mathbf{p})}+\frac{1}{2} E+\frac{1}{n} L_{X(\mathbf{p})}$ логканоничен и численно тривиален над $X$. Имеем

$$
K_{\widetilde{X}(\mathbf{p})}+\widetilde{S}+\frac{1}{2} E_{\widetilde{X}(\mathbf{p})}+\frac{1}{n} L_{\widetilde{X}(\mathbf{p})}=\psi^{*} \phi^{*}\left(K_{X}+\frac{1}{n} L_{X}\right),
$$


где $L_{X}=\phi\left(L_{X(\mathbf{p})}\right)$. Применим $\left(K_{\widetilde{X}(\mathbf{p})}+\widetilde{S}+\left(\frac{1}{2}+\varepsilon\right) E_{\widetilde{X}(\mathbf{p})}+\frac{1}{n} L_{\widetilde{X}(\mathbf{p})}\right)$-ПММ, $0<\varepsilon \ll 1$. Так как $\rho\left(E_{\widetilde{X}(\mathbf{p})}\right)=1$, то на первом и единственном шаге имеем дивизориальное стягивание дивизора $E_{\widetilde{X}(\mathbf{p})}$ в точку.

Итак, получили чисто логтерминальное раздутие $\varphi:(Y, S) \rightarrow(X \ni 0)$ и $\left(S, \operatorname{Diff}_{S}(0)\right)=\left(\overline{\mathbb{F}}_{10}, \frac{1}{2} H_{1}+\frac{2}{3} H_{2}\right)$, где $H_{1}$ и $H_{2}$ - гиперплоские сечения конуса. Дивизор $K_{S}+\operatorname{Diff}_{S}(0)+\frac{1}{6} l$ антиобилен и не логтерминален по Кавамате, где $l-$ образующая конуса, проходящая через точку пересечения (кратности 5) $H_{1}$ и $H_{2}$. По теореме 1.15 особенность $(X, 0)$ не слабо исключительна.

Аналогично рассмотрим оставшиеся случаи для 5-струй $f^{\prime}$. В этих случаях поверхность $S$ не изменяется, $H_{1}$ и $H_{2}$ остаются гиперплоскими сечениями. Изменяются только кратности пересечений в точках $H_{1}$ и $H_{2}$. Теперь теорема получается из следующей несложной леммы.

Лемма 3.4. Логповерхность дель Пеицо $\left(\overline{\mathbb{F}}_{10}, \frac{1}{2} H_{1}+\frac{2}{3} H_{2}\right)$ является исключительной (слабо исключительной) и 6-дополняемой тогда и только тогда, когда гиперплоские сечения $H_{1}$ и $H_{2}$ пересекаются во всех точках с кратностью не более 3 (не более 4).

Следуюшая теорема описывает все исключительные строго логканонические особенности в случае 2) теоремы 3.2 .

Teорема 3.5. Пусть $(X, 0)=\left(f=t^{3}+g_{2}^{2}(z, x, y)+f_{\geqslant 5}^{\prime}(z, x, y)=0,0\right) \subset$ $\left(\mathbb{C}^{4}, 0\right)$ - строго логканоническая особенность. Тогда струя $f_{5}^{\prime}$ равна нулю и $f_{6}^{\prime}$ не равна нулю. Особенность $(X, 0)$ является исключительной и 1-дополняемой тогда и только тогда, когда $f_{6}^{\prime}$ не имеет неприводимого множителя кратности 6. В этом случае существует чисто логтерминальное раздутие $\varphi:(Y, S) \rightarrow(X \ni 0)$ такое, что $\left(S, \operatorname{Diff}_{S}(0)\right)=(S, 0)$, где $S-$ поверхность, полученная стягиванием сечения әллиптической поверхности $S^{\prime} \rightarrow \mathbb{P}^{1}$ в дювалевскую особую точку $\mathbb{A}_{1}\left(\right.$ кодаирова размерность $\left.\kappa\left(S^{\prime}\right)=0\right)$. Вырожденный слой $S^{\prime}$ всегда является әллиптической каспидальной кривой. Особые точки являются дювалевскими особенностями. Они могут лежать только в вершине каспа. В зависимости от неприводимых множителей $f_{6}^{\prime}$ имеем следующие случаи: $12 \mathbb{A}_{0}$, где 12 - иисло вырожденных слоев, а $\mathbb{A}_{0}$ неособая точка; $2 \mathbb{A}_{2}+8 \mathbb{A}_{0} ; 4 \mathbb{A}_{2}+4 \mathbb{A}_{0} ; 6 \mathbb{A}_{2} ; 2 \mathbb{D}_{4}+6 \mathbb{A}_{0} ; 2 \mathbb{D}_{4}+2 \mathbb{A}_{2}+2 \mathbb{A}_{0} ; 4 \mathbb{D}_{4}$; $2 \mathbb{E}_{6}+4 \mathbb{A}_{0} ; 2 \mathbb{E}_{6}+2 \mathbb{A}_{2} ; 2 \mathbb{E}_{8}+2 \mathbb{A}_{0}$.

ДокАЗАТЕльСтво. Доказательство аналогично доказательству теоремы 3.3. Рассмотрим р-раздутие $\phi:(X(\mathbf{p}), E) \rightarrow X$ такое, что $\mathbf{p}=(4,3,3,3)$. Тогда $\left(E, \operatorname{Diff}_{E}(0)\right)=\left(t+g_{2}^{2} \subset \mathbb{P}(4,1,1,1), \frac{2}{3}\{t=0\}\right)=\left(\mathbb{P}^{2}, \frac{4}{3} C\right)$, где $C-$ неприводимая коника. Дискрепантность $a(E, 0)$ равна нулю. Согласно теореме $3.3 \quad f_{5}^{\prime}=0$. Если $f_{6}^{\prime}=0$, то многочлен $f$ в карте $\mathbb{C}_{t^{\prime}, z^{\prime}, x^{\prime}, y^{\prime}}^{4} / \mathbb{Z}_{3}(2,1,0,0)$ имеет вид $t^{\prime 3}+$ $g_{2}^{2}\left(1, x^{\prime}, y^{\prime}\right)+z^{\prime 9} f^{\prime \prime}$. Эта особенность не логканоническая, поэтому 6-струя $f^{\prime}$ ненулевая. Теперь теорема доказьвается перебором всех случаев для 6-струй. Докажем теорему в двух случаях: $f_{6}^{\prime}=z^{6}+f_{\geqslant 7}^{\prime}(z, x, y), f_{6}^{\prime}=z^{5} x+f_{\geqslant 7}^{\prime}(z, x, y)$, а остальные случаи разбираются аналогично.

Вдоль $C \backslash \bigcup P_{i}$ многообразие $X(\mathbf{p})$ имеет неизолированную строго логканоническую особенность $\left(x_{1}^{3}+x_{1} x_{3}^{2}+x_{2}^{3}=0,0\right) \subset\left(\mathbb{C}_{x_{1}, x_{2}, x_{3}, x_{4}}^{4}, 0\right)$. В первом случае 
$i=1,2$ и многообразие $X(\mathbf{p})$ в окрестности точек $P_{i}$ (точки $P_{i}$ имеют координаты $\left(0,1, y_{i}\right)$ в $\mathbb{P}^{2}$, где $\left.g_{2}\left(0,1, y_{i}\right)=0\right)$ имеет вид

$$
\left(x_{1}^{3} x_{4}^{6}+x_{1} x_{3}^{2}+x_{2}^{3}+f^{\prime \prime \prime}=0,0\right) \subset\left(\mathbb{C}_{x_{1}, x_{2}, x_{3}, x_{4}}^{4}, 0\right),
$$

т.е. $P_{i}$ являются центрами логканонических особенностей $X(\mathbf{p})$. Поэтому по определению особенность неисключительна. Во втором случае $i=1,2,3,4$. Многообразие $X(\mathbf{p})$ в окрестности точек $P_{1}, P_{2}$ имеет вид

$$
\left(x_{1}^{3} x_{4}^{5}+x_{1} x_{3}^{2}+x_{2}^{3}+f^{\prime \prime \prime}=0,0\right) \subset\left(\mathbb{C}_{x_{1}, x_{2}, x_{3}, x_{4}}^{4}, 0\right),
$$

а в окрестностях точек $P_{3}, P_{4}-$ вид

$$
\left(x_{1}^{3} x_{4}+x_{1} x_{3}^{2}+x_{2}^{3}+f^{\prime \prime \prime}=0,0\right) \subset\left(\mathbb{C}_{x_{1}, x_{2}, x_{3}, x_{4}}^{4}, 0\right) .
$$

Раздуем $\mathbb{C}^{4}(\mathbf{p})$ вдоль кривой $C$ с весами $(1,1,1,0)$. Тогда это раздутие индуцирует $\psi: \widetilde{X}(\mathbf{p}) \rightarrow X(\mathbf{p})$ и имеем $K_{\widetilde{X}(\mathbf{p})}+S^{\prime}=\psi^{*} K_{X(\mathbf{p})}$. Очевидно, что $f^{\prime \prime \prime}$ не влияет на вклеенную эллиптическую поверхность $S^{\prime} \rightarrow \mathbb{P}^{1}$. Легко проверяется, что над точками $P_{i}$ находятся вырожденные слои. Поверхность $S^{\prime}$ имеет особенности $\mathbb{E}_{8}$ в слоях для $i=1,2$. Дивизор $K_{X(\mathbf{p})}+S^{\prime}$ чисто логтерминален. Применим $\left(K_{\widetilde{X}(\mathbf{p})}+S^{\prime}+\varepsilon E_{\widetilde{X}(\mathbf{p})}\right)$-ПММ, $0<\varepsilon \ll 1$. Так как $\rho\left(E_{\widetilde{X}(\mathbf{p})}\right)=1$, то на первом и единственном шаге имеем дивизориальное стягивание дивизора $E_{\tilde{X}(\mathbf{p})}$ в точку. Итак, получили искомое чисто логтерминальное раздутие $\varphi:(Y, S) \rightarrow(X \ni 0)$.

Согласно доказательству основной теоремы произведем классификацию трехмерных исключительных квазиоднородных канонических особенностей. Процесс классификации состоит из двух частей.

1. Классификация квазиоднородных многочленов. Каждому такому многочлену поставим в соответствие его вес $\mathbf{p}$. Из основной теоремы вектор $\mathbf{p}$ определен однозначно. Будет показано, что существует конечное число весов и, следовательно, конечное число типов исключительных особенностей.

2. Исследование полученных особенностей на исключительность и нахождение среди всех дополнений дополнения минимального индекса.

Teорема 3.6 [21]. Пусть $(X, 0) \subset\left(\mathbb{C}^{4}, 0\right)$ - каноническая гиперповерхностная особенность, заданная многочленом $f$, и пара $(X, H)$ не логканонична для любого гиперплоского сечения $H$. Тогда $f$ принадлежит одному из следующих типов:

$\left(\Upsilon_{1}\right) \quad f=t^{2}+z^{3}+z f_{1}(x, y)+f_{2}(x, y)$, , əde $\operatorname{deg} f_{1} \geqslant 5, \quad \operatorname{deg} f_{2} \geqslant 7 ;$

$\left(\Upsilon_{2}\right) f=t^{2}+f^{\prime}(z, x, y)$, где $\operatorname{deg} f^{\prime} \geqslant 4$ и 4-струя ненулевая;

$\left(\Upsilon_{3}\right) f=t^{2}+f^{\prime}(z, x, y)$, где $\operatorname{deg} f^{\prime} \geqslant 5$ и 5-струя ненулевая;

$\left(\Upsilon_{4}\right)$ ранг квадратичной части $f$ равен 0 и 3 -струя ненулевая. 
ОПРЕДЕЛЕНИЕ 3.7. Будем придерживаться следующих обозначений: $(X, 0)=$ $(f(t, z, x, y)=0,0) \subset\left(\mathbb{C}_{t, z, x, y}^{4}, 0\right)$ - трехмерная каноническая квазиоднородная гиперповерхностная особенность. Поскольку необходимо классифицировать исключительные особенности, то можно считать (пример 1.8), что пара $(X, H)$, где $H$ произвольное гиперплоское сечение, всегда не логканонична.

Классификацию квазиоднородных частей будем проводить методом "врашения линейки Ньютона" $[1, \S 11]$. Для этого сначала выделим часть типа $\mathscr{M}_{2}$ (см. определение 3.1 ), а затем с помощью простейшей компютерной программы получим искомый список квазиоднородных особенностей. Поэтому основная задача заключается в нахождении частей типа $\mathscr{M}_{2}$.

Рассмотрим сначала особенности типа $\Upsilon_{1}$. Следуюшая теорема описывает эти особенности.

ТЕОРЕМа 3.8. Все исключительные канонические квазиоднородные особенности типа $\Upsilon_{1}$ получаются при вращении плоскости в пространстве $\mathbb{C}_{z, \text { }}^{3}$ проходящей через моном $z^{3}$ и один из следующ,их мономов: $x^{7}, x^{8}, x^{7} y, x^{9}$, $x^{8} y, x^{7} y^{2}, x^{10}, x^{9} y, x^{8} y^{2}, x^{7} y^{3}, x^{11}, x^{10} y, x^{9} y^{2}, x^{8} y^{3}, x^{7} y^{4} u z x^{5}, z x^{5} y, z x^{7}$, $z x^{6} y, z x^{5} y^{2}$. Ограничения на вращение: вес переменной $x$ больше веса переменной $y$ (wt $x \geqslant$ wt $y$ ) и точка $\mathbf{2}=(2,2,2)$ лежит над плоскостью (теорема 2.9).

ДокАЗАТЕЛЬСТво. Заметим, что моном $z x^{6}$ не входит в список, так как мономы $z^{3}, z x^{6}, x^{9}$ лежат на одной прямой. Для удобства выберем веса квазиоднородности $f$ такими, чтобы квазиоднородная степень стала равной 1 . В формулировке теоремы выписаны мономы $x^{m}$ вида $z x^{a} y^{b}, x^{a} y^{b}$, удовлетворяющие следующим условиям: $a \geqslant b$; wt $x \geqslant \operatorname{wt} y$; wt $x+$ wt $y>\frac{1}{6}$ (теорема 2.9 ); особенность, заданная $t^{2}+z^{3}+x^{m}$, не логканонична. Докажем, что эти мономы задают все особенности. Пусть квазиоднородный многочлен $f$ задает исключительную особенность $(X, 0)$ типа $\Upsilon_{1}$ и ни один из его мономов не принадлежит списку (в этом случае wt $x>$ wt $y$ ). Пусть $z x^{a_{1}} y^{b_{1}}$ - моном минимальной степени из $f_{1}(x, y)$, а $x^{a} y^{b}$ моном минимальной степени из $f_{2}(x, y)$. Так как wt $x>$ wt $y$, то в этих мономах степень $x$ является наибольшей по сравнению с оставшимися мономами, а степень $y$, наоборот, минимальной. Если $f_{1}=0$ или $f_{2}=0$, то $f^{\prime}=t^{2}+z^{3}+x^{a} y^{b}$ (или $+z x^{a_{1}} y^{b_{1}}$ ) определяет неисключительную логканоническую особенность, так как для нее существуют два различных вектора из $N_{\mathbb{R}}$ (см. доказательство основной теоремы). Взяв $\mathbf{p}=\left(\frac{1}{2}, \frac{1}{3}, \frac{1}{a}, 1\right)$ или $\mathbf{p}=\left(\frac{1}{2}, \frac{1}{3}, \frac{2}{3 a_{1}}, 1\right)$, имеем $f_{\mathbf{p}}=f^{\prime}$. Следовательно, $(X, 0)$ - неисключительная особенность. Поэтому имеем следуюшие четыре случая и докажем, что они невозможны.

Случай 1. $f^{\prime}=t^{2}+z^{3}+x^{a} y^{b}$ и $f^{\prime \prime}=t^{2}+z^{3}+z x^{a_{1}} y^{b_{1}}$ задают не логканонические особенности. Тогда эти особенности не логканоничны вне $\mathbf{0}$ вдоль $\{t=z=y=0\}$. Поэтому $(X, 0)$ не логканонична вне $\mathbf{0}$ вдоль $\{t=z=y=0\}$. Противоречие.

Случай 2. $f^{\prime}=t^{2}+z^{3}+x^{a} y^{b}$ задает не логканоническую особенность (т.е. $a<b$ и $b \geqslant 7$ ) и $f^{\prime \prime}=t^{2}+z^{3}+z x^{a_{1}} y^{b_{1}}$ задает логканоническую особенность (т.е. $a_{1} \leqslant 4$ и $\left.b_{1} \leqslant 4\right)$. Тогда если $b_{1}=0$, то по определению $\Upsilon_{1}$ получаем $a_{1} \geqslant 5$. 
Противоречие. Имеем систему линейных уравнений

$$
\left\{\begin{aligned}
a \text { wt } x+b \text { wt } y & =1 \\
a_{1} \text { wt } x+b_{1} \text { wt } y & =\frac{2}{3}, \quad d=a b_{1}-b a_{1} \neq 0 .
\end{aligned}\right.
$$

Пусть $a_{1} \geqslant b_{1}$. Достаточно найти такие веса wt $x$, wt $y$, чтобы $f_{\mathbf{p}}=f^{\prime \prime}$, где $\mathbf{p}=(1 / 2,1 / 3$, wt $x$, wt $y)$. Уменьшим вес wt $x$ на $\varepsilon$, a wt $y$ увеличим на $\sigma=a_{1} \varepsilon / b_{1}$ (при этом второе уравнение из системы сохранится). Эти веса будут искомыми. Достаточно проверить, что $\left\langle\mathbf{p}, x^{a} y^{b}\right\rangle>1$, т.е. $-d=-a b_{1}+b a_{1}>0$. Это верно, так как $a / b<1$ и $a_{1} / b_{1} \geqslant 1$.

Пусть $a_{1}<b_{1}$. Если $-d>0$, то особенность $(X, 0)$ также неисключительна. Пусть $d>0$, тогда wt $x=\left(b_{1}-\frac{2}{3} b\right) / d>0$. Отсюда $b_{1}>\frac{2}{3} b \geqslant 14 / 3$. Противоречие.

Случай 3. $f^{\prime}=t^{2}+z^{3}+x^{a} y^{b}$ задает логканоническую особенность (т.е. $a \leqslant 6$ и $b \leqslant 6$ ) и $f^{\prime \prime}=t^{2}+z^{3}+z x^{a_{1}} y^{b_{1}}$ задает не логканоническую особенность (т.е. $a_{1}<b_{1}$ и $\left.b_{1} \geqslant 5\right)$. По той же причине, что в случае $2, b \neq 0$. Если $d>0$, то, как и в случае 2 , находим $\mathbf{p}$ такой, чтобы $f_{\mathbf{p}}=f^{\prime}$. Если $d<0$, то, как и в случае 2 , имеем $b_{1}-\frac{2}{3} b<0$, т.е. $b>\frac{3}{2} b_{1} \geqslant \frac{15}{2}$. Противоречие.

Случай 4. $f^{\prime}=t^{2}+z^{3}+x^{a} y^{b}$ и $f^{\prime \prime}=t^{2}+z^{3}+z x^{a_{1}} y^{b_{1}}$ задают логканонические особенности. Аналогично уменьшим wt $x$ и увеличим wt $y$, чтобы сохранилось первое или второе уравнение системы. В одном из случаев получим $f_{\mathbf{p}}=f^{\prime}$, либо $f_{\mathbf{p}}=f^{\prime \prime}$.

Исследуем особенности на исключительность. Напомним основные сведения о гиперповерхностях во взвешенных проективных пространствах [2]. Наибольший общий делитель чисел $a_{1}, \ldots, a_{n}$ обозначим через $\left(a_{1}, \ldots, a_{n}\right)$.

ПРЕДЛОЖЕНИЕ 3.9. Пусть $\left(a_{1}, \ldots, a_{n}\right)=1 u q_{i}=\left(a_{1}, \ldots, \hat{a}_{i}, \ldots, a_{n}\right)$, əде $a_{i} \in \mathbb{N}$ для всех $i$. Тогда $\mathbb{P}=\mathbb{P}\left(a_{1}, \ldots, a_{n}\right) \cong \mathbb{P}\left(a_{1} / q_{i}, \ldots, a_{i}, \ldots, a_{n} / q_{i}\right)$. Если $q_{i}=1$ для всех $i$, mо $K_{\mathbb{P}} \sim \mathscr{O}_{\mathbb{P}}\left(-\sum a_{i}\right)$ и $\mathscr{O}_{\mathbb{P}}(1)^{n-1}=1 /\left(a_{1} \ldots a_{n}\right) . \quad$. этом случае будем говорить, что веса $\mathbb{P}$ хорошо определены.

ПРЕДЛОЖЕНИЕ 3.10. $B$ тех же предположениях пусть $\left(X_{d} \subset \mathbb{P}\right)=$ $\left(g\left(x_{1}, \ldots, x_{n}\right) \subset \mathbb{P}\left(a_{1}, \ldots, a_{n}\right)\right)$ - әиперповерхность степени d. Тогда $\left(X_{d} \subset\right.$ $\mathbb{P})=\left(g\left(x_{1}, \ldots, x_{i}^{1 / q_{i}}, \ldots, x_{n}\right) \subset \mathbb{P}\left(a_{1} / q_{i}, \ldots, a_{i}, \ldots, a_{n} / q_{i}\right)\right)$. Ecлu $\operatorname{Diff}_{X_{d} / \mathbb{P}}(0)=0$ и веса $\mathbb{P}$ хорошо определеньи, то $K_{X_{d}}=\left.\left(K_{\mathbb{P}}+X_{d}\right)\right|_{X_{d}}=\mathscr{O}_{X_{d}}\left(d-\sum a_{i}\right)$.

ОПРЕДЕЛЕНИЕ 3.11. Гиперповерхность $X_{d} \subset \mathbb{P}$, где веса взвешенного проективного пространства $\mathbb{P}$ хорошо определены, называется хорошо определенной, если выполнено одно из следующих равносильных условий:

1) $\operatorname{codim}_{X_{d}}\left(X_{d} \cap \operatorname{Sing} \mathbb{P}\right) \geqslant 2$;

2) $\operatorname{Diff}_{X_{d} / \mathbb{P}}(0)=0$;

3) для всех $i \neq j$ имеем $\left(a_{1}, \ldots, \hat{a}_{i}, \ldots, \hat{a}_{j}, \ldots, a_{n}\right) \mid d$.

ОПРЕДЕЛЕНИЕ 3.12. Пусть $\mathbf{p}=\left(a_{1}, \ldots, a_{n}\right)$ - вес квазиоднородной логканонической особенности $g\left(x_{1}, \ldots, x_{n}\right)=0$, причем $a_{i} \in \mathbb{N}$ для всех $i$. По основной теореме эти веса определены однозначно для исключительной особенности (в частности, $a_{i}>0$ для всех $\left.i\right)$ и $\mathbf{p}$-раздутие индуцирует чисто логтерминальное раздутие 
$(Y, E) \rightarrow(\{g=0\}, 0)$. Полученное логмногообразие Фано является следуюшим:

$$
\begin{aligned}
\left(E, \operatorname{Diff}_{E}(0)\right)= & (E, D)=\left(\tilde{g}\left(x_{1}, \ldots, x_{n}\right) \subset \mathbb{P}\left(\tilde{a}_{1}, \ldots, \tilde{a}_{n}\right), D\right) \\
= & \left(g ( x _ { 1 } ^ { 1 / q _ { 1 } } , \ldots , x _ { n } ^ { 1 / q _ { n } } ) \subset \mathbb { P } \left(a_{1} q_{1} /\left(q_{1} \ldots q_{n}\right), \ldots\right.\right. \\
& \left.\left.\ldots, a_{n} q_{n} /\left(q_{1} \ldots q_{n}\right)\right), \sum \frac{q_{i}-1}{q_{i}}\left\{x_{i}=0\right\}\right) .
\end{aligned}
$$

При подсчете $(E, D)$ существенно, чтобы гиперповерхность $E \subset \mathbb{P}$ являлась хорошо определенной. Если она не хорошо определена, а веса $\tilde{a}_{1}, \ldots, \tilde{a}_{n}$ хорошо определены, то имеем другую дифференту $D$ (см. ниже пример 3.27 и [27]). Здесь и в дальнейшем координаты особенности $g=0$ и координаты взвешенного проективного пространства будем обозначать одинаково.

В нашем случае квазиоднородные степени $f$ и $\tilde{f}$ будем также обозначать через $d$ и $\tilde{d}$ соответственно. Полученную логповерхность дель Пецщо будем всегда обозначать через $(E, D)$.

Следующее предложение позволяет свести вопрос об исключительности полученной логповерхности дель Пецщо к перебору конечного числа случаев.

ПрЕДЛОЖЕНИЕ 3.13 [24, теорема 2.3]. Пусть $(S, \Delta)$ - слабая логповерхность дель Пецио, т.е. дивизор $-\left(K_{S}+\Delta\right)$ численно әффективен и облемен. Предположим, что все 1-, 2-, 3-, 4- и 6-дополнения $\Delta^{+}$являются исключительньми, т.е. пара $\left(S, \Delta^{+}\right)$логтерминальна по Кавамате. Тогда логповерхность $(S, \Delta)$ является исключительной.

Используя определение 1.3, получим следующее следствие.

СлЕДСТВИЕ 3.14. В обозначениях предложения 3.13 пусть дивизор $K_{S}+$ $\frac{\ulcorner(n+1) \Delta\urcorner}{n}$ обилен для всех $n=1,2,3,4,6$. Тогда поверхность $(S, \Delta)$ является исключительной.

СлЕДСТВИЕ 3.15. Пусть для трехмерной квазиоднородной логканонической особенности $(X, 0)$ любое гиперплоское сечение имеет не логканонические особенности и $\left(E, D=\sum d_{i} D_{i}\right)$ - логповерхность дель Пеццо, полученная при чисто логтерминальном раздутии. Если $d_{k} \geqslant \frac{6}{7}$ для некоторого $k u$ $\operatorname{Diff}_{E / \mathbb{P}}(0)=0$, то особенность является исключительной.

ДоКАЗАТЕЛЬСТво. Пусть особенность неисключительна; тогда по предложению 3.13 существует 1-, 2-, 3-, 4- или 6-дополнение $D^{+}$. По определению дополнения $D^{+} \geqslant D_{k}+\sum_{i \neq k} d_{i} D_{i}$, где $D_{k}=\left\{x_{k}=0\right\}$. Так как $\operatorname{Diff}_{E / \mathbb{P}}(0)=0$, то

$$
\frac{1}{q_{1} \ldots q_{4}}\left(d-\sum_{i \neq k} a_{i}\right)=\tilde{d}-\sum_{i \neq k} \frac{\widetilde{a}_{i}}{q_{i}} \leqslant 0 .
$$

Поэтому по теореме 2.9 общее гиперплоское сечение $x_{k}+h\left(x_{1}, \ldots, x_{4}\right)=0$ особенности $(X, 0)$ будет логканоническим. Противоречие. 
ЗАМЕЧАНИЕ 3.16. Гипотетически следствие 3.15 остается верным в любой размерности $n$. В условии положим $d_{k} \geqslant \frac{\left|R N_{n-1}\right|}{\left|R N_{n-1}\right|+1}$, где $\left|R N_{n}\right|=\max \{m \mid m \in$ $\left.R N_{n}\right\}$, а множество $R N_{n}$ определим следуюшим образом: $m \in R N_{n}$, если сушествует неисключительное логмногообразие Фано размерности $n$, у которого минимальный индекс неисключительного дополнения равен $m$. Гипотеза заключается в том, что $\left|R N_{n}\right|<\infty$ для всех $n$. Для $n \leqslant 3$ это доказано; более того, $R N_{1}=\{1,2\}$ и $R N_{2}=\{1,2,3,4,6\}[24]$.

Выделим общие принципы исследования на исключительность.

1. Прежде всего, убедимся, что Diff $E / \mathbb{P}(0)=0$ (удобнее проверить условие 3 ) определения 3.11).

2. Если в дивизор $D$ входит компонента с коэффициентом $\geqslant \frac{6}{7}$, то по следствию 3.15 особенность исключительна. Любое дополнение $D^{+}$для пары $(E, D)$ можно продолжить до дополнения $K_{\mathbb{P}}+E+\widehat{D}^{+} \sim_{\mathbb{Q}} 0$, где $\widehat{D}^{+} \mid E=D^{+}$. Отсюда и из строения групшы дивизоров Вейля взвешенного проективного пространства $\mathbb{P}$ следует, что Supp $D^{+}$высекается полиномами.

Пусть

$$
r_{n}=\sum_{i=1}^{4} \tilde{a}_{i}-\tilde{d}-\frac{1}{n} \sum_{i=1}^{4}\left\ulcorner\frac{q_{i}-1}{q_{i}}(n+1)\right\urcorner \tilde{a}_{i},
$$

т.е. $K_{E}+\frac{\ulcorner(n+1) D\urcorner}{n} \sim_{\mathbb{Q}}-r_{n} \mathscr{O}_{E}(1)$. Тогда $r_{n} \geqslant 0$ и $r_{n}=\frac{1}{n}\left(\sum \tilde{a}_{i} b_{i}\right)$, где $b_{i} \in \mathbb{N}$ для всех $i$. Для числа $n \in \mathbb{N}$ со свойством (*) будет существовать $n$-дополнение.

3. По следствию 3.13 возможны два случая: $(E, D)$ - исключительная поверхность; сушествует 1-, 2-, 3-, 4- или 6-дополнение. В дальнейшем предполагаем, что имеет место второй случай. Тогда сначала определим особые точки $\tilde{f} \subset \mathbb{P}$ и особенности кривых из $\operatorname{Supp} D$. Для этого самое простое - воспользоваться леммой 2.2. Результат зависит от коэффициентов $f$.

4. Пусть $D^{+}$- дополнение для $(E, D)$. По теореме 1.14 вопрос об исключительности равносилен вопросу: логтерминальна ли пара $\left(E, D^{+}\right)$по Кавамате или нет? Так как этот вопрос локальный, то рассмотрим эту пару в карте $U_{k}=\mathbb{C}^{3} / \mathbb{Z}_{a_{k}}\left(a_{1}, \ldots, a_{k-1}, a_{k+1}, \ldots, a_{n}\right)$ для $\mathbb{P}$. Так как логтерминальность по Кавамате сохраняется при накрытиях [9, предложение 3.16], то исследование будет сводиться к конечному числу вопросов: логтерминальна ли пара $\left(\mathbb{C}_{u, v}^{2}, B=\sum c_{i}\left\{h_{i}(u, v)=0\right\}\right)$ по Кавамате или нет? пара $\left(\mathbb{C}_{u, v, w}^{3}, B=\right.$ $\left.\{h=0\}+\sum c_{i}\left\{h_{i}(u, v)=0\right\}\right)$, где $h=0$ определяет дювалевскую особенность ( $h$ - локальное уравнение $E$ в соответствующей карте), чисто логтерминальна или нет? Для ответа на эти вопросы удобно рассмотреть аналог индуктивного раздутия [11, теорема 1.5] (это такое раздутие с единственным исключительным

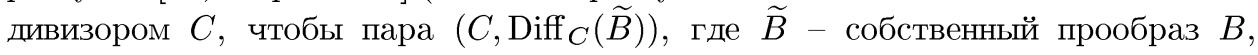
оказалась логтерминальной по Кавамате или чисто логтерминальной) и воспользоваться леммой 3.20 .

Проведем исследование на исключительность особенностей типа $\Upsilon_{1}$.

Рассмотрим далее наиболее интересные случаи и обшие примеры, иллюстрирующие исследование полученных логповерхностей дель Пещо на исключительность. 
Пример 3.17. $f=t^{2}+z^{3}+x^{11}+y^{13}$. Тогда $(E, D)=\left(\mathbb{P}^{2}, \frac{1}{2} l_{1}+\frac{2}{3} l_{2}+\frac{10}{11} l_{3}+\frac{12}{13} l_{4}\right)$, где $l_{i}$ - прямые в общем положении. По следствию 3.14 или 3.15 полученная логповерхность является исключительной. Минимальный индекс дополняемости равен 66 , соответствуюшее дополнение $D^{+}$равно $\frac{1}{2} l_{1}+\frac{2}{3} l_{2}+\frac{10}{11} l_{3}+\frac{61}{66} l_{4}$. Гипотетически это максимальное число для логповерхностей и, следовательно, для всех трехмерных локальных стягиваний (особенностей) [24]. Другими словами, $\left|R N_{3}\right|=66$ (см. замечание 3.16$)$.

ПримеР 3.18. $f=t^{2}+z^{3}+g(z, x, y)=t^{2}+z^{3}+z y f_{2}^{\prime}\left(x^{2}, y^{3}\right)+x f_{3}^{\prime}\left(x^{2}, y^{3}\right)$. Нижние индексы обозначают степени бинарных форм. Эти формы являются различными, хотя и обозначаются одинаково через $f^{\prime}$. Заметим, что перед тем, как выписать $f$, с помощью замены $z \longmapsto z+\alpha x y^{2}$ для некоторого $\alpha$ избавимся от монома $z^{2} x y^{2}$. Врашение происходит через единственную часть $t^{2}+z^{3}+x^{7}$ типа $\mathscr{M}_{2}$. Поэтому коэффициент при $x^{7}$ не равен нулю. Положим $f_{2}^{\prime}=a x^{2 i} f_{2-i}\left(x^{2}, y^{3}\right)$, $f_{3}^{\prime}=x^{2 j} f_{3-j}\left(x^{2}, y^{3}\right)$.

ПРЕДЛОЖЕНИЕ 3.19. Особенность $(X, 0)$ исключительна и 2-дополняема тогда и только тогда, когда $i j=0$ (при $a=0$ считаем, что $i \neq 0)$.

ДокАЗАТЕльство. Чисто логтерминальное раздутие с весами $(21,14,6,4)$ дает

$$
(E, D)=\left(t+z^{3}+g(z, x, y) \subset \mathbb{P}(21,7,3,2), \frac{1}{2}\{t=0\}\right)=\left(\mathbb{P}(7,3,2), \frac{1}{2}\{t=0\}\right)
$$

Пусть $i \geqslant 1$ и $j \geqslant 1$. Тогда дивизор $K_{E}+\frac{1}{2}\{t=0\}+\frac{1}{2}\{x=0\} \equiv 0$ не логтерминален по Кавамате и, следовательно, особенность неисключительна. Проверим это. Пусть $i=j=1$. Тогда это утверждение равносильно тому, что пара $\left(\mathbb{C}_{u, v}^{2}, \frac{1}{2}\left\{\left(u^{3}+a^{\prime} u^{2} v+v^{3}\right) v=0\right\}\right)$ не логтерминальна по Кавамате. Действительно, при раздутии с весами $(1,1)$ появится дивизор с дискрепантностью -1 . Случаи с другими $i, j$ разбираются аналогично.

Теперь пусть $i=0$. Рассмотрим два наиболее "плохих" случая:

1) $g(z, x, y)=z y^{7}+x^{7}$

2) $g(z, x, y)=z y\left(x^{2}+y^{3}\right)^{2}+x\left(x^{2}+y^{3}\right)^{3}$.

Первый случай, очевидно, исключительный. Во втором случае надо проверить, что дивизор $K_{E}+\frac{1}{2}\{t=0\}+\frac{1}{4}\left\{x^{2}+y^{3}=0\right\} \equiv 0$ логтерминален по Кавамате. Это утверждение равносильно тому, что пара $\left(\mathbb{C}_{u, v}^{2}, B\right)$, где $B=\frac{1}{2}\left\{u^{3}+a^{\prime} u^{2} v+\right.$ $\left.v^{3}=0\right\}+\frac{1}{4}\{v=0\}$, логтерминальна по Кавамате. Для доказательства этого или аналогичного утверждения удобно воспользоваться следующей очевидной леммой.

ЛЕмма 3.20. Пусть для пары $(Z \ni P, D)$ исключительное множество бирачионального морфизма $f: Y \rightarrow Z$ состоит из одного исключительного дивизора $C$. Тогда если $а(C, D)>-1(=-1)$ и дивизор $K_{Y}+C+D_{Y}$, әде $D_{Y}$ - собственный прообраз $D$, чисто логтерминален, то пара $(Z \ni P, D)$ логтерминальна по Кавамате (логканонична) и исключительна.

При раздутии с весами $(1,1)$ появится дивизор $C \cong \mathbb{P}^{1}$ с дискрепантностью $-\frac{3}{4}$, и дивизор $K_{C}+\operatorname{Diff}_{C}(\widetilde{B})=K_{C}+\frac{1}{2} P_{1}+\frac{1}{2} P_{2}+\frac{1}{2} P_{3}+\frac{1}{4} P_{4}$ будет логтерминальным 
по Кавамате; здесь $P_{i}$ - различные точки и $\widetilde{B}$ - собственный прообраз $B$. Случай $j=0$ разбирается аналогично. Дивизор $D^{+}=\frac{1}{2}\{t=0\}+\frac{1}{2}\{x=0\}$ дает 2-дополнение.

Рассмотрим факторособенность $\mathbb{C}^{3} / H_{168}$, где $H_{168}$ - простая группа Клейна порядка 168. Указанная особенность имеет тот же тип, что и в этом примере, и является исключительной (ср. [18]). Отметим, что это - вырожденная гиперповерхностная особенность.

ПримеР 3.21. $f=t^{2}+z^{3}+\underline{a} z x^{i} f_{5-i}^{\prime}\left(x, y^{2}\right)+\underline{b} y x^{j} f_{7-j}^{\prime}\left(x, y^{2}\right)$. Для этой особенности сушествуют две различные части типа $\mathscr{M}_{2}$, через которые происходит вращение: $t^{2}+z^{3}+x^{7} y, t^{2}+z^{3}+z x^{5}$. Поэтому коэффициент при $z x^{5}$ или при $x^{7} y$ не равен нулю. Относительно записи $f$ см. соглашения в $\S 4$. Чисто логтерминальное раздутие с весами $(15,10,4,2)$ дает $(E, D)=\left(t+z^{3}+\cdots \subset\right.$ $\left.\mathbb{P}(15,5,2,1), \frac{1}{2}\{t=0\}\right)=\left(\mathbb{P}(5,2,1), \frac{1}{2}\{t=0\}\right)$. Пусть $i \geqslant 3$ и $j \geqslant 5$. Тогда дивизор $K_{E}+\frac{1}{2}\{t=0\}+\frac{1}{4}\{x=0\} \equiv 0$ не логтерминален по Кавамате и, следовательно, особенность неисключительна. Действительно, рассмотрим $(E, D)$ в окрестности точки $(0: 0: 0: 1)$. Тогда надо доказать, что пара $\left(\mathbb{C}^{2}, \frac{1}{2}\left\{u^{3}+\underline{a} u v^{i}+\underline{b} v^{j}=0\right\}+\frac{1}{4}\{v=0\}\right)$ не логтерминальна по Кавамате. При раздутии с весами $(3,2)$ появится дивизор с дискрепантностью -1 , что и требовалось доказать.

Если $i \leqslant 2$ или $j \leqslant 4$, то особенность исключительна и 2-дополняема. Условие $i \leqslant 2|| j \leqslant 4$ также означает, что оно вьполнено для других обших компонент вида $x+c y^{2}$. Это условие выписывается только для $x$, так как после квазиоднородной замены $x \longmapsto x^{\prime}-c y^{2}$ мы приходим к исходному случаю. Рассмотрим два наиболее "плохих" случая. Пусть $j=4$ и $\underline{a}=0$. Достаточно показать, что пара $\left(\mathbb{C}^{2}, B=\right.$ $\left.\frac{1}{2}\left\{u^{3}+v^{4}=0\right\}+\frac{1}{4}\{v=0\}\right)$ логтерминальна по Кавамате. При раздутии $\mathrm{c}$ весами $(4,3)$ появится дивизор $C$ с дискрепантностью $-3 / 4$. Пара $\left(C, \operatorname{Diff}_{C}(\widetilde{B})\right)=$ $\left(\mathbb{P}^{1}, \frac{1}{2} P_{1}+\frac{7}{8} P_{2}+\frac{2}{3} P_{3}\right)$ логтерминальна по Кавамате, где $P_{i}$ - различные точки. По лемме 3.20 все доказано. Оставшийся "плохой” случай $i=2$ и $\underline{b}=0$ разбирается аналогично.

Рассмотрим особенности типа $\Upsilon_{2}$. Для исключительных особенностей этого типа по следствию 2.19 существует биголоморфное отображение, после которого 4-струи могут быть только следуюшими: $z^{4}$ (тип $\Upsilon_{2}^{(1)}$ ), $z^{3} x$ (тип $\Upsilon_{2}^{(2)}$ ), $z^{3} y$ (тип $\left.\Upsilon_{2}^{(3)}\right)$.

1) Особенности типа $\Upsilon_{2}^{(1)}:\left(t^{2}+z^{4}+z^{2} f_{1}(x, y)+z f_{2}(x, y)+f_{3}(x, y)\right)$. Для вращения линейки Ньютона (условие на врашение $\mathrm{wt} x \geqslant \operatorname{wt} y$ ) достаточно зафиксировать мономы только следуюших видов: $x^{a} y^{b}$ и $z x^{a} y^{b}$. Аналогично, как и для особенностей типа $\Upsilon_{1}$ (теорема 3.8 ), имеем следующие случаи: $x^{5}, x^{6}, x^{5} y, x^{7}, x^{6} y$, $x^{5} y^{2}$ и $z x^{4}, z x^{5}, z x^{4} y$.

ПримеР 3.22. $f=t^{2}+z^{4}+z x^{4} y+x^{3} y^{n}, n=4,5$. Веса для чисто логтерминального раздутия - это $(2(4 n-3),(4 n-3), 3 n-4,7)$. Далее, $(E, D)=(f \subset \mathbb{P}(2(4 n-$ $3),(4 n-3), 3 n-4,7), 0)$ и $K_{E}=(n-6) \mathscr{O}_{E}(1)$. При $n=5$ нет 1-, 2-, 3-, 4- и 6-дополнения. Поэтому особенность исключительна и $\frac{1}{7}\{y=0\}$ дает 7 -дополнение минимального индекса. Пусть $n=4$. Тогда имеется 4 -дополнение $D^{+}=\frac{1}{4}\{x=0\}$. 
Докажем, что пара $\left(E, D^{+}\right)$не логтерминальна по Кавамате. Для этого достаточно показать, что пара $\left(\mathbb{C}_{u, v, w}^{3},\left\{u^{2}+v^{4}+w^{3}=0\right\}+\frac{1}{4}\{w=0\}\right)$ не чисто логтерминальна. При взвешенном раздутии с весами $(6,3,4)$ для этой пары появится дивизор с дискрепантностью -1 . Поэтому особенность неисключительна.

2) Особенности типа $\Upsilon_{2}^{(2)}:\left(t^{2}+z^{3} x+g(z, x, y)\right)$. Аналогично, как и для особенностей типа $\Upsilon_{1}$ (теорема 3.8 ), имеем следующие случаи для вращения линейки Ньютона (wt $x \geqslant$ wt $y$ ): $x^{5}, x^{6}, x^{5} y, x^{7}, x^{6} y, x^{5} y^{2}, x^{8}, x^{7} y, x^{6} y^{2}, x^{5} y^{3}, x^{9}, x^{8} y$, $x^{7} y^{2}, x^{6} y^{3}, x^{5} y^{4}$ и $z x^{4}, z x^{4} y, z x^{6}, z x^{5} y, z x^{4} y^{2}$. Отметим, что случай $z x^{5}$ входит в случай $x^{7}$.

ПримеР 3.23. $f=t^{2}+z^{3} x+a z^{2} y x^{i_{1}}\left(x+y^{3}\right)^{i_{2}} f_{2-i_{1}-i_{2}}\left(x, y^{3}\right)+b z y^{2} x^{j_{1}}(x+$ $\left.y^{3}\right)^{j_{2}} f_{3-j_{1}-j_{2}}\left(x, y^{3}\right)+x^{l_{1}}\left(x+y^{3}\right)^{l_{2}} f_{5-l_{1}-l_{2}}\left(x, y^{3}\right)$. Нижние индексы обозначают степени однородности многочлена. С помощью замены $x \longmapsto x+\alpha y^{3}$ для некоторого $\alpha$ избавимся от $z^{3} y^{3}$. Вращение происходит через единственную часть $t^{2}+z^{3} x+x^{5}$ типа $\mathscr{M}_{2}$. Поэтому коэффициент при $x^{5}$ не равен нулю. Так как $x$ входит в моном $z^{3} x$, то выделяем другую общую часть $x+y^{3}$. Чисто логтерминальное раздутие - это раздутие с весами $(15,8,6,2)$. Тогда $(E, D)=\left(\mathbb{P}(4,3,1), \frac{1}{2}\{t=0\}\right)$. Имеем следуюшие 1-, 2-, 3-, 4- и 6-дополнения: 2-дополнение $D+\frac{1}{2}\{y=0\}$ и 6-дополнение $D+\frac{1}{6}\left\{x+\beta y^{3}=0\right\}$. Минимальный индекс дополняемости для исключительной особенности равен 2. Так как кривая $\{y=0\}$ не проходит через особые точки $\operatorname{Supp} D$, то первое дополнение можно не рассматривать. Рассмотрим оставшиеся случаи $\beta=0, \beta=1$.

Пусть $\beta=0$. Тогда при $i_{1} \geqslant 2\left(i_{1}>2\right.$ означает, что $\left.a=0\right), j_{1} \geqslant 3\left(j_{1}>3\right.$ означает, что $b=0), l_{1}=3$ (при $l_{1} \geqslant 4$ исходная особенность не каноническая) пара $\left(\mathbb{C}_{u, v}^{2}, B\right)$, где $B=\frac{1}{2}\left\{u^{3} v+v^{3}+a u^{2} v^{2}+b u v^{3}=0\right\}+\frac{1}{6}\{v=0\}$, не логтерминальна по Кавамате. Действительно, при раздутии с весами $(2,3)$ появится дивизор с дискрепантностью - 1 . Во всех остальных случаях это дополнение будет исключительным. Докажем это, например, для $i_{1} \geqslant 2, j_{1}=2, l_{1} \geqslant 3$. В этом случае $B=\frac{1}{2}\left\{u^{3} v+a u^{2} v^{2}+u v^{2}+v^{l_{3}}=0\right\}+\frac{1}{6}\{v=0\}$. Сделаем раздутие с весами $(1,2)$. Дискрепантность при исключительном дивизоре $C$ равна $-\frac{5}{6}$, при этом $\operatorname{Diff}_{C}(\widetilde{B})=\frac{3}{4} P_{1}+\frac{2}{3} P_{2}+\frac{1}{2} P_{3}$, где $\widetilde{B}-$ собственный прообраз $B$. Отсюда дивизор $K_{C}+\operatorname{Diff}_{C}(\widetilde{B})$ логтерминален по Кавамате, т.е. по лемме 3.19 пара $\left(\mathbb{C}^{2}, B\right)$ логтерминальна по Кавамате.

Пусть $\beta=1$. Тогда $B=\frac{1}{2}\{h=0\}+\frac{1}{6}\{v=0\}$, где $h(u, v)=u^{3}+a u^{2} v^{i_{2}}+$ $b u v^{j_{2}}+v^{l_{2}}$. Легко проверить, что при $b \neq 0$ пара $\left(\mathbb{C}^{2}, B\right)$ логтерминальна по Кавамате. Пусть $b=0$. Аналогично проверяется, что при $i_{2} \leqslant 1$ пара $\left(\mathbb{C}^{2}, B\right)$ всегда логтерминальна по Кавамате. Пусть $i_{2} \geqslant 2$. Тогда при $l_{2} \leqslant 4$ эта пара логтерминальна по Кавамате, а при $l_{2}=5$ - не логтерминальна по Кавамате.

Итак, каноническая особенность исключительна и 2-дополняема, кроме случая $i_{1} \geqslant 2 \& j_{1} \geqslant 3 \& l_{1}=3$ и случая $i_{2} \geqslant 2 \& b=0 \& l_{1}=3$. Условия всегда выписываются с учетом других общих компонент (см. пример 3.20$)$.

3) Особенности типа $\Upsilon_{2}^{(3)}$. Справедлива следуюшая теорема.

ТЕОРЕМА 3.24. Все исключительные квазиоднородные канонические особенности этого типа получаются при вращении плоскости в пространстве $\mathbb{C}_{z, x, y}^{3}$, проходящей через моном $z^{3} y$ и один из следующих мономов: $x^{7}, x^{8}$, 
$x^{7} y, x^{9}, x^{8} y, x^{7} y^{2} u z x^{5}, z x^{6}, z x^{5} y, z^{2} x^{3}$. Орраничения на вращение: вес переменной $x$ больше веса переменной $y$ (wt $x>$ wt $y$ ) и точка $\mathbf{2}=(2,2,2)$ лежит над плоскостью.

ДоказАТеЛЬСТво. Пусть $f=t^{2}+z^{3} y+z^{2} f_{1}(x, y)+z f_{2}(x, y)+z^{2} f_{3}(x, y)$. Обозначим через $z^{2} x^{a} y^{b}$ моном минимальной степени из $z^{2} f_{1}(x, y)$. Если $b \neq 0$, то в силу квазиоднородной биголоморфной замены $z \longmapsto z-\alpha f_{1}(x, y) y^{-1}$ можно считать, что $f_{1}(x, y)=0$. Дальнейшее доказательство аналогично доказательству теоремы 3.8 . Пусть $b=0$. Тогда, используя wt $x+\mathrm{wt} y+\mathrm{wt} z>1 / 2$, несложно получить, что $a \leqslant 3$. Из следствия 2.19 получаем, что $a=3$, что и требовалось доказать.

Рассмотрим особенности типа $\Upsilon_{3}$. Для исключительных особенностей этого типа рассмотрим три возможных случая для 5-струй.

I. 5-струя является точкой (тип $\mathscr{M}_{0}$ ), т.е. $f_{5}=z^{a} x^{b} y^{c}$, где $a \geqslant b, a \geqslant c$ и $a+b+c=5$. Из следствия 2.19 имеем $a \geqslant 3$. Итак, 5-струя является одной из следуюших: $z^{5}$ (тип $\Upsilon_{3}^{(1)}$ ), $z^{4} x$ (тип $\Upsilon_{3}^{(2)}$ ), $z^{4} y$ (тип $\Upsilon_{3}^{(3)}$ ), $z^{3} x^{2}$ (тип $\Upsilon_{3}^{(4)}$ ), $z^{3} y^{2}$ (тип $\Upsilon_{3}^{(5)}$ ), $z^{3} x y$ (тип $\left.\Upsilon_{3}^{(6)}\right)$. Аналогично теоремам $3.8,3.24$ имеем следуюшие случаи для врашения линейки Ньютона (wt $x \geqslant$ wt $y$ ).

1) Особенности типа $\Upsilon_{3}^{(1)}$. Мономы для вращения: $x^{6}, x^{5} y, x^{4} y^{2}$ и $z x^{5}, z x^{4} y$, $z x^{3} y^{2}$.

2) Особенности типа $\Upsilon_{3}^{(2)}$. Мономы для вращения: $x^{6}, x^{5} y, x^{4} y^{2}$ и $z x^{5}, z x^{4} y$, $z x^{3} y^{2}$.

3) Особенности типа $\Upsilon_{3}^{(3)}$. Мономы для вращения (wt $x>$ wt $y$ ): $x^{6}, x^{5} y$ и $z x^{5}$, $z x^{4} y$. Все особенности в случае $z x^{4} y$ не являются хорошо определенными.

4) Особенности типа $\Upsilon_{3}^{(4)}$. Мономы для вращения: $x^{6}, x^{5} y, x^{4} y^{2}, x^{3} y^{3}, x^{7}, x^{6} y$, $x^{5} y^{2}, x^{4} y^{3}, x^{3} y^{4}$ и $z x^{5}, z x^{4} y, z x^{3} y^{2}$.

5) Особенности типа $\Upsilon_{3}^{(5)}$. Мономы для вращения (wt $\left.x>\operatorname{wt} y\right): x^{7}$ и $z x^{5}$.

6) Особенности типа $\Upsilon_{3}^{(6)}$. Мономы для вращения: $x^{5}, x^{6}, x^{5} y, x^{7}, x^{6} y, x^{5} y^{2}$ и $z x^{5}, z x^{4} y$. Все особенности в случаях $x^{5} y, x^{6} y, z x^{4} y$ не являются хорошо определенными.

II. 5-струя - прямая, т.е. $f_{5} \in \mathscr{M}_{1}$ (тип $\left.\Upsilon_{3}^{(7)}\right)$. Для вращения линейки Ньютона (wt $x \geqslant$ wt $y$ ) имеем следуюшие случаи: $g_{5}(z, x)$ - бинарная форма степени $5, z^{5}+$ $z x^{3} y, z^{5}+x^{4} y, z^{4} x+z x^{3} y, z^{4} x+x^{4} y, z^{4} y+z^{2} x^{3}, z^{3} x^{2}+z x^{3} y, z^{3} x^{2}+x^{3} y^{2}$, $z^{3} y^{2}+z^{2} x^{3}$. Этот список был получен следующим образом. В первый моном $x^{m_{1}}$ входит переменная в наибольшей степени, которую обозначим через $z$. Второй моном $x^{m_{2}}=z^{a} x^{b} y^{c}$ выписан из условий, что $b \geqslant c$ и особенность $t^{2}+x^{m_{1}}+x^{m_{2}}$ не логканонична вне $\mathbf{0 .}$

Доказательство того, что этими случаями описьваются все возможности, аналогично теореме 3.8. Рассмотрим одномерную 5-струю $f_{5}=z^{5}+\alpha z^{3} x y+\beta z x^{2} y^{2}$. Докажем, что ее можно не рассматривать. Если $\beta \neq 0$, то $\exists \mathbf{p} \in N_{\mathbb{R}}$ такой, что $f_{\mathbf{p}}=t^{2}+f_{5}$ - логканоническая вне $\mathbf{0}$ особенность, поэтому по следствию 2.19 исходная особенность неисключительна. Действительно, пусть $x^{n}=z^{c} x^{a} y^{b}$ (где $c+a+b \geqslant 6)-$ другой моном и $d=b-a$ - определитель системы линейных 
уравнений, определяющих веса

$$
\left\{\begin{aligned}
\text { wt } x+\text { wt } y & =\frac{2}{5} \\
a \text { wt } x+b \text { wt } y & =1-\frac{1}{5} c .
\end{aligned}\right.
$$

Если $d<0$, то из условия, что wt $x \geqslant$ wt $y$, получаем $c+a+b \leqslant 5$. Противоречие. Пусть $d>0$. Уменьшим вес wt $x$ на $\varepsilon$, а вес wt $y$ увеличим на $\varepsilon$, тогда для соответствуюшего им вектора $\mathbf{p}$ имеем $\left\langle\mathbf{p}, x^{n}\right\rangle>1$. Пусть теперь $\beta=0$ и $x^{n}=z^{c} x^{a} y^{b}$ (где $c+a+b \geqslant 6$ ) - другой моном. Аналогично получается, что $d>0$. Из условий wt $x>0$ и wt $y>0$ при $c=0,1$ имеем $b \geqslant 4$, а при $c=2,3$ имеем $b \geqslant 3$, т.е. особенность $t^{2}+z^{3} x y+x^{n}$ не логканонична вне 0 вдоль $\{t=z=x=0\}$. Следовательно, исходная особенность не логканонична.

Все остальные случаи рассматриваются аналогично, за исключением случая $f_{5}=z^{4} y+z^{3} x^{2}$. Тогда, перебирая все варианты, имеем только два новых: $t^{2}+$ $z^{4} y+z^{3} x^{2}+x y^{5}, t^{2}+z^{4} y+z^{3} x^{2}+a z y^{5}+b x^{2} y^{4} \quad(b \neq 0)$. Легко проверить, что они неисключительны.

III. 5-струя - плоскость, т.е. $f_{5} \in \mathscr{M}_{2}\left(\right.$ тип $\left.\Upsilon_{3}^{(8)}\right)$. Классификация таких особенностей сводится к описанию всех кривых степени 5 в $\mathbb{P}^{2}$.

ТЕОРема 3.25. Пусть $f=t^{2}+f_{5}(z, x, y)$ задает каноническую особенность $(X, 0)$, где $f_{5}$ - однородный многочлен степени 5. Тогда р-раздутие, где $\mathbf{p}=(5,2,2,2)$, индуиирует чисто логтерминальное раздутие $(X, 0)$, причем $(E, D)=\left(t+f_{5}(z, x, y) \subset \mathbb{P}(5,1,1,1), \frac{1}{2}\{t=0\}\right)=\left(\mathbb{P}^{2}, \frac{1}{2} C\right)$, әде $C=\left\{f_{5}=0\right\}$. Особенность $(X, 0)$ является исключительной только в следующих случаях:

1) кривая $C \subset \mathbb{P}^{2}$ является неприводимой; ее особыми точками могут быть только обыкновенные каспь и обыкновенные двойные точки;

2) кривая $C \subset \mathbb{P}^{2}$ распадается на неприводимую кубику и неприводимую конику, которые пересекаются в шести различных точках; особая точка кубики (если она есть) может быть только обыкновенным каспом или обыкновенной двойной точкой.

ДоказАТЕльСтво. Так как особенность $(X, 0)$ нормальная, то каждая неприводимая компонента кривой $C=\left\{f_{5}=0\right\}$ имеет кратность 1. Отсюда имеем три случая: $C$ - неприводимая кривая, кривая $C$ распадается на неприводимую кубику и неприводимую конику, кривая $C$ распадается на прямую и квартику. Логповерхность дель Пеццо $(E, D)=\left(\mathbb{P}^{2}, \frac{1}{2} C\right)$. В первом случае, если особенность хуже обыкновенного каспа, легко проверить, что дивизор $K_{\mathbb{P}^{2}}+\frac{1}{2} C+\frac{1}{2} l \sim_{\mathbb{Q}} 0$, где прямая $l$ проходит через особенность, не логтерминален по Кавамате (в случае особенностей $\left(u^{2}+v^{n}=0,0\right) \subset\left(\mathbb{C}^{2}, 0\right)$, где $n=4,5$ и $\left.l=\{u=0\}\right)$. Во втором случае, если кубика и коника пересекаются с кратностью 2 в точке $P$, та же пара будет не логтерминальной по Кавамате, где прямая $l$ касается коники в точке $P$. В третьем случае в качестве кривой $l$ достаточно взять прямую из $\operatorname{Supp} C$. Теорема доказана. 
ПримеР 3.26. $f=t^{2}+z^{3} x y+x^{i}\left(x+y^{2}\right)^{j} f_{5-i-j}\left(x, y^{2}\right)=t^{2}+z^{3} x y+$ $h(x, y)$. Врашение происходит через единственную прямую, заданную многочленом $t^{2}+z^{3} x y+x^{5}$. Поэтому коэффициент при $x^{5}$ не нулевой. Чисто логтерминальное раздутие - это раздутие с весами $(15,7,6,3)$. Тогда $(E, D)=\left(t^{2}+z x y+\right.$ $\left.h(x, y) \subset \mathbb{P}(5,7,2,1), \frac{2}{3}\{z=0\}\right)$. Имеем следующие 1-, 2-, 3-, 4- и 6-дополнения: 3 -дополнение $D+\frac{1}{3}\{y=0\}$ и 6-дополнение $D+\frac{1}{6}\left\{x+\beta y^{2}=0\right\}$. Если $i \geqslant 1$, то 6 -дополнение при $\beta=0$ является 3-дополнением $D+\frac{1}{3} C$, где $C=\{t=x=0\}$. Минимальный индекс дополняемости для исключительной особенности равен 3. Аналогично примеру 3.23 достаточно рассмотреть второе дополнение.

Пусть $\beta=0$. Тогда при $i=3$ (при $i_{1} \geqslant 4$ особенность не каноническая) особенность неисключительна. Действительно, надо показать, что пара $\left(\mathbb{C}_{u, v, w}^{3}, B\right)$ не чисто логтерминальна, где $B=\left\{u^{2}+v w+w^{3}=0\right\}+\frac{2}{3}\{v=0\}+\frac{1}{6}\{w=0\}$. При раздутии с весами $(3,4,2)$ появляется дивизор с дискрепантностью -1 . Если $i \leqslant 2$, то пара $\left(E, D+\frac{1}{6}\{x=0\}\right)$ логтерминальна по Кавамате. Докажем это для $i=2$. Надо показать, что пара $(Z, B)$ логтерминальна по Кавамате, где $Z=\left\{u^{2}+v w+w^{2}=0\right\} \subset\left(\mathbb{C}^{3}, 0\right)$ и $B=\left.\frac{2}{3}\{v=0\}\right|_{Z}+\left.\frac{1}{6}\{w=0\}\right|_{Z}$. При раздутии с весами $(1,1,1)$ появляется дивизор $C$ с дискрепантностью $a(C, B)=-\frac{5}{6}$ и $\operatorname{Diff}_{C}(\widetilde{B})=\frac{2}{3} P_{1}+\frac{2}{3} P_{2}+\frac{1}{3} P_{3}$. Отсюда дивизор $K_{C}+\operatorname{Diff}_{C}(\widetilde{B})$ логтерминален по Кавамате, что и требовалось доказать.

Пусть $\beta=1$. Тогда аналогично проверяется, что особенность исключительна тогда и только тогда, когда $j \leqslant 4$.

Итак, каноническая особенность исключительна и 3 -дополняема только при $i \leqslant 2$ и $j \leqslant 4$.

Рассмотрим особенности типа $\Upsilon_{4}$. Эти особенности рассматриваются аналогично предыдущим. Имеем для вращения линейки Ньютона (wt $x \geqslant \operatorname{wt} y$ ) четьре случая. Для подчеркнутых частей все особенности не являются хорошо определенными или они входят в другие случаи.

(А) $f_{3} \in \mathscr{M}_{0}$. Тогда $f_{3}$ будет равна одному из следующих мономов: $t^{3}, t^{2} z, t^{2} x$, $t^{2} y$. Рассмотрим по отдельности каждый из них.

1) $\mathrm{C}$ точностью до замены координат $f=t^{3}+t g(z, x, y)+h(z, x, y)$. Так как $\mathbf{1} \in \Gamma_{+}(f)^{0}$, то $f_{4} \neq 0$. Имеем следующие случаи: $z^{2} x^{2}+x^{5}, z^{2} x^{2}+x^{4} y, z^{2} x^{2}+x^{3} y^{2}$, $z^{2} x^{2}+x^{2} y^{3} ; z^{2} x y+x^{5}, z^{2} x y+x^{4} y ; f_{4}=\varphi_{4}(z, x)$ - бинарная форма степени $4, z^{4}+$ $z x^{2} y, z^{4}+x^{3} y, z^{4}+t x^{3}, z^{4}+t x^{2} y ; z^{3} x+z x^{2} y, z^{3} x+x^{3} y, z^{3} x+t x^{3}, z^{3} x+t x^{2} y ; z^{3} y+$ $z^{2} x^{2}, z^{3} y+t x^{3} ; t z^{3}+z^{2} x^{2}, \underline{t z^{3}+z x^{3}}, t z^{3}+z x^{2} y, \underline{t z^{3}+x^{3} y} ; z^{2} x^{2}+a z x^{2} y+b x^{2} y^{2}$,

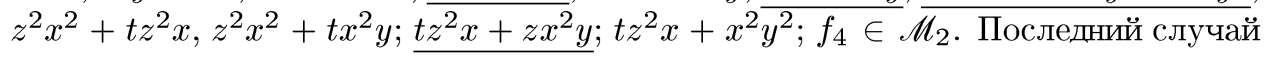
разобран в теореме 3.29 .

2) $\mathrm{C}$ точностью до замены координат $f=t^{2} z+t g(x, y)+h(z, x, y)$. Тогда также $f_{4} \neq 0$. Имеем следующие случаи: $f_{4}=\varphi_{4}(z, x)$ - бинарная форма степени 4 , $z^{4}+x^{3} y, z^{4}+x^{5}, z^{4}+x^{4} y, z^{4}+x^{3} y^{2}, z^{4}+t x^{3}, z^{4}+t x^{2} y ; z^{3} x+x^{3} y, z^{3} x+x^{5}$, $z^{3} x+x^{4} y, z^{3} x+x^{3} y^{2}, z^{3} x+t x^{3}, z^{3} x+t x^{2} y, z^{3} y+x^{5} ; z^{3} y+x^{4} y, z^{3} y+z x^{3}, z^{3} y+z^{2} x^{2}$, $z^{3} y+t x^{3} ; z^{2} x^{2}+x^{5}, z^{2} x^{2}+x^{4} y, z^{2} x^{2}+x^{3} y^{2}, z^{2} \overline{x^{2}+t x^{2} y} ; z^{2} y^{2}+x^{5} ; z^{2} x y+x^{5}$, $z^{2} x y+x^{4} y$.

3) $\mathrm{C}$ точностью до замены координат $f=t^{2} x+t g(z, y)+h(z, x, y)$. Тогда также $f_{4} \neq 0$. Имеем следуюшие случаи: $z^{4}+x^{3} y, z^{4}+x^{5}, z^{4}+x^{4} y, z^{4}+$ $x^{3} y^{2}, z^{4}+z x^{2} y, z^{4}+z x^{4}, z^{4}+z x^{3} y, z^{4}+z x^{2} y^{2} ; \underline{z^{3} x+x^{2} y^{2}}, \underline{z^{3} x+x^{5}}, \underline{z^{3} x+x^{4} y}$, 
$\frac{z^{3} x+x^{3} y^{2}}{z^{3} x+z x^{2} y}, \frac{z^{3} x+x^{2} y^{3}}{z^{3} x+z x^{4}}, \frac{z^{3} x+x^{6}}{z^{3} x+z x^{3} y}, \frac{z^{3} x+x^{5} y}{z^{3} x+z x^{2} y^{2}} ; z^{3} y+x^{3}, x^{5}, z^{3} y+x^{4} y, z^{3} y+x^{6}$, $\overline{z^{3} y+x^{5} y,} z^{3} y+x^{4} y^{2}, z^{3} y+z x^{4}, z^{3} y+z x^{3} y, z^{3} y+z^{2} x^{2}$.

4) С точностью до замены координат $f=t^{2} y+t g(z, x)+h(z, x, y)$. Тогда также $f_{4} \neq 0$. Имеем следующие случаи: $z^{4}+x^{5}, z^{4}+z x^{4} ; z^{3} x+x^{5}, z^{3} x+x^{6}, \underline{z^{3} x+x^{5} y}$, $z^{3} x+z x^{4}, z^{3} x+t x^{3}$.

(B) $f_{3} \in \mathscr{M}_{1}$. Тогда функция $f_{3}$ будет одной из следующих:

1) $f_{3}=g_{3}(t, z)$. Мономы для врашения: $x^{4}, x^{5}, x^{4} y, z x^{3}$.

2) $f_{3}=t^{3}+z^{2} x$. Мономы для вращения: $x^{4}, x^{5}, x^{4} y, x^{6}, x^{5} y, x^{4} y^{2}, x^{7}, x^{6} y, x^{5} y^{2}$, $x^{4} y^{3}, x^{8}, x^{7} y, x^{6} y^{2}, x^{5} y^{3}, x^{4} y^{4}, t x^{3}, t x^{3} y, t x^{5}, t x^{4} y, t x^{3} y^{2}$. Мономы $z x^{3}, z x^{4}, z x^{3} y$, $t x^{4}$ входят в случаи $x^{5}, x^{7}, x^{5} y^{2}, x^{6}$ соответственно.

3) $f_{3}=t^{3}+z^{2} y$. Мономы для вращения: $x^{7}, x^{8}, x^{7} y, z x^{4}, t x^{5}$.

4) $f_{3}=t^{2} z+z^{2} x$. Мономы для вращения: $x^{4}, \overline{x^{5},} x^{4} y, x^{6}, x^{5} y, x^{4} y^{2}, t x^{3}, t x^{4}$, $t x^{3} y$. Моном $z x^{3}$ входит в случаи $x^{5}$.

5) $f_{3}=t^{2} z+z^{2} y$. Мономы для врашения: $x^{5}, x^{6}, \underline{x^{5} y}, \underline{z x^{3}}, t x^{4}$.

(C) $f_{3} \in \mathscr{M}_{2}$. Тогда заменой координат функцию $f_{3}$ можно привести к случаю $(\mathrm{A})$ или $(\mathrm{B})$, либо логканонический порог $c\left(f_{3}\right)$ равен 1 . В последнем случае по следствиям $2.18,2.19$ особенность $(X, 0)$ неисключительна.

(D) $f_{3} \in \mathscr{M}_{3}$. Тогда заменой координат функцию $f_{3}$ можно привести к случаю (A), (B) или (C), либо общее гиперплоское сечение имеет логканонические особенности, т.е. особенность $(X, 0)$ неисключительна.

ПримеР 3.27. Многочлен $f$ имеет тип $f_{3}(t, z)+y^{2} f_{2}(t, z)+\left(y^{4}+x^{2} y\right) f_{1}(t, z)+$ $f_{2}\left(x^{2}, y^{3}\right)$. Предполагаем, что бинарная форма $f_{3}(t, z)$ не имеет кратных неприводимых компонент. Эта особенность получается при врашении линейки Ньютона для случая $f_{3}(t, z)+x^{4} \in \mathscr{M}_{2}$. После квазиоднородных замен (см. начало $\left.\S 4\right)$ $f=t^{3}+k t z^{2}+l z^{3}+t\left(a x^{2} y+b y^{4}\right)+z\left(c x^{2} y+d y^{4}\right)+e t z y^{2}+x^{2 i} f_{2-i}\left(x^{2}, y^{3}\right)$. Причем $|k|+|l| \neq 0$. Чисто логтерминальное раздутие - это раздутие с весами $(4,4,3,2)$. Тогда $(E, D)=\left(f\left(t, z, x^{1 / 2}, y\right) \subset \mathbb{P}(2,2,3,1), \frac{1}{2}\{x=0\}\right)$. Имеем следующие 1-, 2-, 3-, 4-, 6-дополнения: $D+\frac{1}{2}\{y=0\} ; \frac{2}{3}\{x=0\} ; D+\frac{1}{4}\left\{\alpha t+\beta z+\gamma y^{2}=0\right\}$; $D+\frac{1}{6}\{y=0\}+\frac{1}{6}\{\alpha t+\beta z=0\}$. Так как коэффищиент при $x^{4}$ не нулевой, то имеет смысл рассматривать только второе и третье дополнения $(\gamma=0)$.

Пусть $e \neq 0$. Тогда при следуюших предположениях: $l=0(k \neq 0) \& z\left(c x^{2} y+\right.$ $\left.d y^{4}\right)=0 \& i=2$ - дивизор $D^{+}=\frac{1}{4}\{t=0\}+\frac{1}{2}\{x=0\}$ дает не логтерминальное по Кавамате 4-дополнение.

Пусть $e=0$. Если $|b|+|d|=0 \& i \geqslant 1$, то $D^{+}=\frac{2}{3}\{x=0\}$ дает не логтерминальное по Кавамате 3 -дополнение. Условие на исключительность, данное при $e \neq 0$, сохранится. Более того, оно обобшится. Дивизор $D^{+}=\frac{1}{4}\{t+\alpha z=0\}+\frac{1}{2}\{x=0\}$ дает не логтерминальное по Кавамате 4 -дополнение, если $i=2 \&$ НОД $\left(t^{3}+k t z^{2}+\right.$ $\left.l z^{3}, t\left(a x^{2} y+b y^{4}\right)+z\left(c x^{2} y+d y^{4}\right)\right)=t+\alpha z$.

ПРИмеР 3.28. $f=t^{2} x+z^{3} y+x^{5}+z x y^{3}$. Чисто логтерминальное раздутие это раздутие с весами $(16,11,8,7)$. Тогда $(E, D)=(f(t, z, x, y) \subset \mathbb{P}(16,11,8,7), 0)$. Рассмотрим карту окрестности точки $(0: 0: 0: 1)$. Тогда пара $\left(\mathbb{C}^{3}, S+\right.$ $\left.\frac{1}{4} T\right)=\left(\mathbb{C}_{u, v, w}^{3},\left\{u^{2} w+v^{3}+w^{5}+v w=0\right\}+\frac{1}{4}\{w=0\}\right)$ не чисто логтерминальна. Для этого надо сделать раздутие $\psi$ с весами $(1,2,4)$. Следовательно, особенность неисключительна. Особенности такого типа устроены нетривиально. Например, 
в нашем примере $(S, 0)$ - это дювалевская особенность типа $\mathbb{A}_{5}$. Поэтому, чтобы доказать исключительность, надо построить индуктивное раздутие. В качестве упражнения покажем, что в этом примере пара $(C, B)=\left(C, \operatorname{Diff}_{C}\left(\frac{1}{4} \widetilde{T}^{\prime}\right)\right)$, где $C-$ исключительная кривая при $\left.\psi\right|_{\widetilde{S}}: \widetilde{S} \rightarrow S$, а $\widetilde{T}^{\prime}-$ собственньй прообраз $T^{\prime}=\left.T\right|_{S}$, логтерминальна по Кавамате. Кривая $(C \subset \mathbb{P})=\left(u w+v^{3}+v w \subset \mathbb{P}(1,1,2)\right)$ не является хорошо определенной. Поэтому для подсчета $(C, B)$ не выполняются условия определения 3.12. Особенности $\widetilde{S}$-это $\left(u^{2}+v^{3}+v \subset \mathbb{C}_{u, v, w}^{3}\right) / \mathbb{Z}_{4}(-1,2,1)=\mathbb{A}_{3}$ и $\left(u^{2} w+1+w \subset \mathbb{C}_{u, v, w}^{3}\right) / \mathbb{Z}_{2}(1,1,0)=\mathbb{A}_{1}$. Так как $\operatorname{deg}_{C} \widetilde{T}^{\prime}=3$ и $\widetilde{T}^{\prime} \cap \operatorname{Sing} \widetilde{S}=\varnothing$, то $(C, B)=\left(\mathbb{P}^{1}, \frac{3}{4} P_{1}+\frac{1}{2} P_{2}+\frac{3}{4} P_{3}\right)$, что и требовалось доказать.

ТЕорема 3.29. Пусть $f=t^{3}+f_{4}(z, x, y)$ задает каноническую особенность $(X, 0)$, где $f_{4}$ - однородный многочлен степени 4 . Тогда р-раздутие, где $\mathbf{p}=(4,3,3,3)$, индуцирует чисто логтерминальное раздутие $(X, 0)$, причем $(E, D)=\left(t+f_{4}(z, x, y) \subset \mathbb{P}(4,1,1,1), \frac{2}{3}\{t=0\}\right)=\left(\mathbb{P}^{2}, \frac{2}{3} C\right)$, әде $C=\left\{f_{4}=0\right\}$. Особенность $(X, 0)$ является исключительной только в следующих случаях:

1) кривая $C \subset \mathbb{P}^{2}$ является неприводимой; ее особыми точками могут быть только обыкновенные двойные точки;

2) кривая $C \subset \mathbb{P}^{2}$ распадается на две неприводимые коники, которые пересекаются в четырех различных точках.

ДокАЗАТЕЛЬСТво такое же, как и в теореме 3.25 .

\section{§ 4. Классификация трехмерных исключительных канонических гиперповерхностных особенностей}

Приведем комментарии к таблицам. Классификация исключительных особенностей дана в таблищах следующего вида. В первом столбце записан номер особенности. Во втором столбце записаны уравнение особенности, условия на исключительность и дополняемость. Почти во всех случаях выписывается сразу серия многочленов, которые были сгруппированы по похожим логповерхностям дель Пецц $(E, D)$ (см. 3.12). В третьей колонке выписаны сами $(E, D)$. В верхней строчке записана хорошо определенная гиперповерхность $E \subset \mathbb{P}$. Если $E$ является линейньгм конусом (т.е $\tilde{d}=\tilde{a}_{k}$ для некоторого $k$ ), то во второй строчке выписано $E=\mathbb{P}\left(\tilde{a}_{1}, \ldots, \tilde{a}_{k-1}, \tilde{a}_{k+1}, \ldots, \tilde{a}_{n}\right)$. Так же во второй строчке выписана дифферента $D$, которая будет обозначаться через Diff $=\left(\frac{q_{1}-1}{q_{1}}, \frac{q_{2}-1}{q_{2}}, \frac{q_{3}-1}{q_{3}}, \frac{q_{4}-1}{q_{4}}\right)$. В четвертом столбце выписан минимальный индекс дополняемости. Вернемся ко второму столбцу. В нем различные случаи разделены либо запятой, либо точкой с запятой. Если стоит запятая, то следуюшая особенность имеет такой же индекс дополняемости. Знак “;” означает, что следуюшая особенность имеет другой индекс дополняемости, выписанный в четвертом столбце правее (или ниже). Также в описании различных случаев используются обычные логические символы: \& "и", $\|$ - "или". Например, запись $|(n=4 ; 5) \& b \neq 0| \ldots|2,4|$ означает, что в случае $n=4$ и $b \neq 0$ имеем 2 -дополнение, а в случае $n=5$ и $b \neq 0$ имеем 4-дополнение. Знак ||| означает тоже логическое "или", но используется только для разделения многочленов (мономов). Например, запись $h+\left(x^{8}|| x^{7} y\right)$ означает, что рассматриваем отдельно два случая: $h+x^{8}, h+x^{7} y$. Для них условия на исключительность также разделены знаком $\| \mid$. 
Очень часто не выписываются условия на каноничность. Читатель легко может сделать это сам. Неизолированные компоненты особенности определяются с помощью леммы 2.2. По теореме 2.9 особенность канонична тогда и только тогда, когда вдоль всех компонент имеем дювалевские особенности. Также надо учитьвать требование на нормальность особенности: $\operatorname{codim}_{\mathbb{C}^{4}} \operatorname{Sing} X \geqslant 3$. Например, особенность $\left(t^{3}-t g(z, x, y)+a g^{3}(z, x, y)=0,0\right)$ является ненормальной, если $a=\frac{4}{3 \sqrt{3}}$, $\frac{2}{3 \sqrt{3}}$.

Если известен минимальный индекс дополняемости, то искомое дополнение элементарно восстанавливается.

Пусть дана квазиоднородная особенность с весом $\mathbf{p}$ и степенью $d$. Может случиться, что этому взвешиванию соответствует большое количество мономов. На квазиоднородной части взвешивания естественно действует группа квазиоднородных биголоморфных отображений $H(\mathbf{p})$. Поэтому задача сводится к классификации орбит действия и нахождению наиболее простого элемента из каждой орбиты. Общий метод, которым это можно сделать, описан в [1]. Группа $H(\mathbf{p})$ порождается торической и унипотентной частями. Торическая часть - это растяжение координат. Унипотентная часть - это квазиоднородные замены вида $\alpha \longmapsto \alpha+h(\beta, \gamma, \delta)$. В нашем случае особенности не очень сложные, поэтому легко догадаться, какие преобразования надо применять. В таблицах приведен наиболее удобный вид для особенности. Для него производится разделение только на те орбиты, которые имеют другое условие на исключительность.

В связи со сказанным выше введем следуюшие соглашения.

(А) Если $g(z, x, y)$ не зависит от $z$, то иногда пишем сокрашено $g(x, y)$. В третьем столбце, ради экономии места, часто не выписывается $g\left(z^{1 / q_{2}}, x^{1 / q_{3}}, y^{1 / q_{4}}\right)$ в подробном виде.

(В) Запись во втором столбце вида $n=3 i \leqslant 2,4 i \leqslant 1$ означает, что при $n=3$ условие на исключительность $i \leqslant 2$, а при $n=4$ условие на исключительность $i \leqslant 1$ (при этом индекс дополняемости один и тот же).

(C) Запись $i, j \leqslant 1$ равносильна $i \leqslant 1 \& j \leqslant 1$.

(D) $f_{n}(x, y)$ - бинарная форма степени $n$. В записи уравнения особенности часто встречаются сразу несколько бинарных форм, которые обозначаются одинаково, например через $f$. Будем считать, что они различны, т.е. не зависят друг от друга. Рассмотрим запись $x^{a i} f_{n-i}\left(x^{a}, y^{b}\right)$. Для нее предполагаем, что коэффициент при мономе $x^{a n}$ ненулевой. Если нет коэффициента перед бинарной формой, то всегда будем считать, что коэффициент при мономе $x^{a n}$ ненулевой. Пусть $a=1$ и оставшаяся часть $f^{\prime}$ особенности не зависит от $x$ (т.е. $\operatorname{HOД}\left(f^{\prime}, x\right)=1$ ). Тогда считаем, что неприводимые множители $f_{n-i}\left(x, y^{b}\right)$ имеют кратность не более $i$.

(Е) Рассмотрим запись $x^{i}\left(x+y^{b}\right)^{j} f_{n-i-j}\left(x, y^{b}\right)$. Пусть оставшаяся часть $f^{\prime}$ особенности зависит от $x$ и не зависит от $x+y^{b}$ (т.е. НОД $\left(f^{\prime}, x+y^{b}\right)=1$ ). Тогда считаем, что неприводимые множители $f_{n-i-j}\left(x, y^{b}\right)$ имеют кратность не более $j$.

(F) Рассмотрим запись $c x^{a i}\left(x^{a}+y^{b}\right)^{j} f_{n-i-j}\left(x^{a}, y^{b}\right)$. Коэффициент $c$ означает, что коэффициенты бинарной формы произвольные. Если $i+j>n$, то $c=0$. Если $i+j \leqslant n$, то $c \neq 0$. Если в условии на исключительность стоит $c \neq 0$, то считаем, что коэффищиент $c$ отсутствует (другими словами, коэффищиент при мономе $x^{a n}$ ненулевой). Пусть $a=1$. Если оставшаяся часть особенности не зависит от $x$, то 
неприводимые множители $f_{n-i}\left(x, y^{b}\right)$ имеют кратность не более $i$, а если зависит от $x$, но не от $x+y^{b}$, то неприводимые множители имеют кратность не более $j$.

(I) Может случиться, что квазиоднородный многочлен имеет более одной части типа $\mathscr{M}_{2}$, принадлежащих списку для вращения линейки Ньютона. Они будут иметь общую часть типа $\mathscr{M}_{1}$ и отличаться только мономами. В нетривиальных случаях их коэффициенты будут подчеркнуты. Пусть подчеркнуто $k$ коэффициентов. Тогда необходимо рассмотреть $k$ случаев, в каждом из которых соответствующий коэффищиент отсутствует. Это имеет большое значение для бинарных форм (cм. (D)-(F)).

(J) Если в первом столбце (во второй строке) стоит сноска, то условие на исключительность приведено в конще соответствующей таблицы.

(K) Условия на исключительность выписываются сразу для всех обших компонент.

ПримеР 4.1. 1) $f=t^{2}+z^{3}+\underline{a} z x^{i} f_{5-i}\left(x, y^{2}\right)+\underline{b} y x^{j} f_{7-j}\left(x, y^{2}\right)$. Условие на исключительность $i \leqslant 2 \| j \leqslant 4$ (см. пример 3.21) означает также следуюшее. Предположим, что $x+c y^{2}$ входит с кратностью $i_{1}$ в $f_{5-i}\left(x, y^{2}\right)$ и с кратностью $j_{1}$ в $f_{7-j}\left(x, y^{2}\right)$. Тогда условие на исключительность означает, что $i_{1} \leqslant 2 \| j_{1} \leqslant 4$. Напомним, что если $\underline{a}=0$ или $\underline{b}=0$, то согласно п. (F) считаем, что $i>5$ или $j>8$ соответственно.

2) $f=t^{2}+z^{3} x+x^{i}\left(x+y^{2}\right)^{j} f_{5-i}\left(x, y^{2}\right)$. Условие на исключительность $i \leqslant$ $1 \& j \leqslant 3$. Здесь, в отличие от предыдущего примера, оставшаяся часть $t^{2}+z^{3} x$ зависит от $x$. Поэтому условие $i \leqslant 1$ фиксировано, а требование $j \leqslant 3$, как и в предыдушем пункте, означает, что кратность любой неприводимой компоненты $f_{5-i}\left(x, y^{2}\right)$ не превосходит 3 .

ТАБлицА 1. Особенность: $t^{2}+z^{3}+g(z, x, y)$

\begin{tabular}{|c|c|c|c|}
\hline № & $g(z, x, y)$ & Логповерхность & Индекс \\
\hline 1 & $\begin{array}{c}x^{7}+a z y^{4 n}+b y^{6 n} \\
n=2,3,4,5,6\end{array}$ & $\begin{array}{c}t^{2}+z^{3}+x+a z y^{4}+b y^{6} \subset \mathbb{P}(3,2,6,1) \\
\mathbb{P}(3,2,1), \text { Diff }=\left(0,0, \frac{6}{7}, \frac{n-1}{n}\right)\end{array}$ & 7 \\
\hline 2 & $\begin{array}{c}x^{7}+a z y^{2 n}+b y^{3 n} \\
n=3 ; 5 ; 9,11,13\end{array}$ & $\begin{array}{c}t+z^{3}+x+a z y^{2}+b y^{3} \subset \mathbb{P}(3,1,3,1) \\
\mathbb{P}(3,1,1), \text { Diff }=\left(\frac{1}{2}, 0, \frac{6}{7}, \frac{n-1}{n}\right)\end{array}$ & $7,8,14$ \\
\hline 3 & $\begin{array}{c}x^{7}+y^{2 n}, n=4,5 ; 8 \\
10,11,13 ; 16,17,19,20\end{array}$ & $\begin{array}{c}t^{2}+z+x+y^{2} \subset \mathbb{P}(1,2,2,1) \\
\mathbb{P}(1,2,1), \text { Diff }=\left(0, \frac{2}{3}, \frac{6}{7}, \frac{n-1}{n}\right)\end{array}$ & $\begin{array}{c}7,9,15 \\
21\end{array}$ \\
\hline 4 & $\begin{array}{c}x^{7}+y^{n} \\
n=11 ; 13 ; 17 ; 19 ; \\
23 ; 25 ; 29 ; 31 ; 37,41\end{array}$ & $\begin{array}{l}t+z+x+y \subset \mathbb{P}(1,1,1,1) \\
\mathbb{P}^{2}, \text { Diff }=\left(\frac{1}{2}, \frac{2}{3}, \frac{6}{7}, \frac{n-1}{n}\right)\end{array}$ & $\begin{array}{c}12,14,18, \\
21,24,28, \\
30,36,42\end{array}$ \\
\hline 5 & $\begin{array}{c}x^{i} f_{7-i}\left(x, y^{2}\right) \\
i \leqslant 3\end{array}$ & $\begin{array}{c}t^{2}+z+x^{i} f_{7-i}\left(x, y^{2}\right) \subset \mathbb{P}(7,14,2,1) \\
\mathbb{P}(7,2,1), \text { Diff }=\left(0, \frac{2}{3}, 0,0\right)\end{array}$ & 3 \\
\hline 6 & $\begin{array}{c}a z y^{2} x^{i} f_{4-i}\left(x, y^{3}\right)+ \\
x^{j} f_{7-j}\left(x, y^{3}\right), i \leqslant 3 \| j \leqslant 4\end{array}$ & $\begin{array}{c}t+z^{3}+g(z, x, y) \subset \mathbb{P}(21,7,3,1) \\
\mathbb{P}(7,3,1), \text { Diff }=\left(\frac{1}{2}, 0,0,0\right)\end{array}$ & 2 \\
\hline 7 & $\begin{array}{c}x^{i} f_{7-i}\left(x, y^{4}\right) \\
i \leqslant 5\end{array}$ & $\begin{array}{c}t^{2}+z+x^{i} f_{7-i}\left(x, y^{2}\right) \subset \mathbb{P}(7,14,2,1) \\
\mathbb{P}(7,2,1), \text { Diff }=\left(0, \frac{2}{3}, 0, \frac{1}{2}\right)\end{array}$ & 3 \\
\hline
\end{tabular}


Продолжение таблицы 1

\begin{tabular}{|c|c|c|c|}
\hline 8 & $\begin{array}{c}x^{i} f_{7-i}\left(x, y^{5}\right) \\
i \leqslant 5\end{array}$ & $\begin{array}{c}t+z+x^{i} f_{7-i}(x, y) \subset \mathbb{P}(7,7,1,1) \\
\mathbb{P}(7,1,1), \text { Diff }=\left(\frac{1}{2}, \frac{2}{3}, 0, \frac{4}{5}\right)\end{array}$ & 6 \\
\hline 9 & $\begin{array}{l}x^{7}+a x^{4} y^{n}+b x y^{2 n} \\
n=5 \quad b \neq 0 ; 7,11 ; 13,17\end{array}$ & $\begin{array}{c}t+z+x^{7}+a x^{4} y+b x y^{2} \subset \mathbb{P}(7,7,1,3) \\
\mathbb{P}(7,1,3), \text { Diff }=\left(\frac{1}{2}, \frac{2}{3}, 0, \frac{n-1}{n}\right)\end{array}$ & $6,12,18$ \\
\hline 10 & $\begin{array}{c}x^{7}+a x^{4} y^{2 n}+b x y^{4 n} \\
n=2 \quad b \neq 0 ; 4,5,7,8\end{array}$ & $\begin{array}{c}t^{2}+z+g\left(z, x, y^{1 / n}\right) \subset \mathbb{P}(7,14,2,3) \\
\mathbb{P}(7,2,3), \text { Diff }=\left(0, \frac{2}{3}, 0, \frac{n-1}{n}\right)\end{array}$ & 3,9 \\
\hline 11 & $\begin{array}{c}x^{7}+x y^{n} \\
n=7 ; 11 ; 13 ; 17 ; 19 \\
23 ; 25,29 ; 31,35\end{array}$ & $\begin{array}{c}t+z+x^{7}+x y \subset \mathbb{P}(7,7,1,6) \\
\mathbb{P}(7,1,6), \text { Diff }=\left(\frac{1}{2}, \frac{2}{3}, 0, \frac{n-1}{n}\right)\end{array}$ & $\begin{array}{c}8,12,15, \\
18,22,24 \\
30,36\end{array}$ \\
\hline 12 & $\begin{array}{c}x^{2 i+1} f_{3-i}\left(x^{2}, y^{n}\right) \\
n=5 i \leqslant 1 ; 7,11\end{array}$ & $\begin{array}{l}t+z+g\left(z, x, y^{1 / n}\right) \subset \mathbb{P}(7,7,1,2) \\
\mathbb{P}(7,1,2), \text { Diff }=\left(\frac{1}{2}, \frac{2}{3}, 0, \frac{n-1}{n}\right)\end{array}$ & 6,12 \\
\hline 13 & $\begin{array}{c}a z y^{n} x^{2 i} f_{2-i}\left(x^{2}, y^{3 n}\right)+ \\
+x^{2 j+1} f_{3-j}\left(x^{2}, y^{3 n}\right), n=1 \\
i j=0 ; n=3 i \leqslant 1|| j \leqslant 2\end{array}$ & $\begin{array}{c}t+z^{3}+g\left(z, x, y^{1 / n}\right) \subset \mathbb{P}(21,7,3,2) \\
\mathbb{P}(7,3,2), \text { Diff }=\left(\frac{1}{2}, 0,0, \frac{n-1}{n}\right)\end{array}$ & 2,4 \\
\hline 14 & $\begin{array}{c}x^{7}+x^{2} y^{n}, n=7 ; 11 \\
13 ; 17 ; 19,23 ; 29\end{array}$ & $\begin{array}{c}t+z+x^{7}+x^{2} y \subset \mathbb{P}(7,7,1,5) \\
\mathbb{P}(7,1,5), \text { Diff }=\left(\frac{1}{2}, \frac{2}{3}, 0, \frac{n-1}{n}\right)\end{array}$ & $\begin{array}{l}9,12,16 \\
18,24,30\end{array}$ \\
\hline 15 & $\begin{array}{c}x^{7}+a z x^{3} y^{2 n}+b x^{2} y^{6 n} \\
n=2 b \neq 0 ; 3,4\end{array}$ & $\begin{array}{c}t^{2}+z^{3}+g\left(z, x, y^{1 / n}\right) \subset \mathbb{P}(21,14,6,5) \\
\text { Diff }=\left(0,0,0, \frac{n-1}{n}\right)\end{array}$ & 4,5 \\
\hline 16 & $\begin{array}{c}x^{7}+a z x^{3} y^{n}+b x^{2} y^{3 n} \\
n=3 \quad b \neq 0 ; 7,9\end{array}$ & $\begin{array}{c}t+z^{3}+g\left(z, x, y^{1 / n}\right) \subset \mathbb{P}(21,7,3,5) \\
\mathbb{P}(7,3,5), \text { Diff }=\left(\frac{1}{2}, 0,0, \frac{n-1}{n}\right)\end{array}$ & 4,10 \\
\hline 17 & $\begin{aligned} & x^{7}+x^{2} y^{2 n} \\
n= & 4,7,8 ; 11,13,14\end{aligned}$ & $\begin{array}{c}t^{2}+z+x^{7}+x^{2} y^{2} \subset \mathbb{P}(7,14,2,5) \\
\mathbb{P}(7,2,5), \text { Diff }=\left(0, \frac{2}{3}, 0, \frac{n-1}{n}\right)\end{array}$ & 9,15 \\
\hline 18 & $\begin{array}{c}x^{7}+a z x^{2} y^{2 n}+ \\
+b x^{3} y^{3 n}, n=3,5,7\end{array}$ & $\begin{array}{c}t+z^{3}+g\left(z, x, y^{1 / n}\right) \subset \mathbb{P}(21,7,3,4) \\
\mathbb{P}(7,3,4), \text { Diff }=\left(\frac{1}{2}, 0,0, \frac{n-1}{n}\right)\end{array}$ & 8 \\
\hline 19 & $\begin{array}{c}x^{7}+x^{3} y^{n} \\
n=7 ; 11 ; 13,17 ; 19,23\end{array}$ & $\begin{array}{c}t+z+x^{7}+x^{3} y \subset \mathbb{P}(7,7,1,4) \\
\mathbb{P}(7,1,4), \text { Diff }=\left(\frac{1}{2}, \frac{2}{3}, 0, \frac{n-1}{n}\right)\end{array}$ & $\begin{array}{c}10,12,18 \\
24\end{array}$ \\
\hline 20 & $\begin{array}{c}x^{7}+z y^{n}, n=5 ; 9 ; 11 \\
13 ; 15 ; 17,19 ; 23,25,27\end{array}$ & $\begin{array}{c}t+z^{3}+x+z y \subset \mathbb{P}(3,1,3,2) \\
\mathbb{P}(1,3,2), \text { Diff }=\left(\frac{1}{2}, 0, \frac{6}{7}, \frac{n-1}{n}\right)\end{array}$ & $\begin{array}{l}7,10,14, \\
16,22,28\end{array}$ \\
\hline 21 & $\begin{array}{c}x^{7}+z x y^{2 n}, n=3,4,5 ; \\
6,7,8,9,10\end{array}$ & $\begin{array}{c}t^{2}+z^{3}+x^{7}+z x y^{2} \subset \mathbb{P}(21,14,6,11) \\
\text { Diff }=\left(0,0,0, \frac{n-1}{n}\right)\end{array}$ & 5,11 \\
\hline 22 & $\begin{array}{c}x^{7}+z x y^{n}, n=5,7 ; 9 \\
13,15 ; 17,19,21\end{array}$ & $\begin{array}{l}t+z^{3}+x^{7}+z x y \subset \mathbb{P}(21,7,3,11) \\
\mathbb{P}(7,3,11), \text { Diff }=\left(\frac{1}{2}, 0,0, \frac{n-1}{n}\right)\end{array}$ & $\begin{array}{c}8,10,16 \\
22\end{array}$ \\
\hline 23 & $\begin{array}{c}x^{7}+z x^{2} y^{n}, n=5,7,9 \\
11,13,15\end{array}$ & $\begin{array}{c}t+z^{3}+x^{7}+z x^{2} y \subset \mathbb{P}(21,7,3,8) \\
\mathbb{P}(7,3,8), \text { Diff }=\left(\frac{1}{2}, 0,0, \frac{n-1}{n}\right)\end{array}$ & 10,16 \\
\hline 24 & $\begin{array}{c}x^{i} y^{j} f_{6-i-j}(x, y) \\
i=j=1\end{array}$ & $\begin{array}{c}t^{2}+z+g(z, x, y) \subset \mathbb{P}(4,8,1,1) \\
\mathbb{P}(4,1,1), \text { Diff }=\left(0, \frac{2}{3}, 0,0\right)\end{array}$ & 3 \\
\hline 25 & $\begin{array}{c}x^{8}+a z y^{2 n}+b y^{3 n} \\
n=3,5 ; 7\end{array}$ & $\begin{array}{c}t^{2}+z^{3}+x^{2}+a z y^{2}+b y^{3} \subset \mathbb{P}(3,2,3,2) \\
\text { Diff }=\left(0,0, \frac{3}{4}, \frac{n-1}{n}\right)\end{array}$ & 5,8 \\
\hline
\end{tabular}


Продолжение таблицы 1

\begin{tabular}{|c|c|c|c|}
\hline 26 & $\begin{array}{c}x^{8}+y^{n} \\
n=11 ; 13 ; 17 ; 19 ; 23\end{array}$ & $\begin{array}{c}t^{2}+z+x^{2}+y \subset \mathbb{P}(1,2,1,2) \\
\mathbb{P}(1,1,2), \text { Diff }=\left(0, \frac{2}{3}, \frac{3}{4}, \frac{n-1}{n}\right)\end{array}$ & $\begin{array}{c}12,13,18 \\
21,24\end{array}$ \\
\hline 27 & $\begin{array}{c}a z y^{3} x^{4 i} f_{1-i}\left(x^{4}, y^{9}\right)+ \\
x^{4 j} f_{2-j}\left(x^{4}, y^{9}\right), i=0 \| j=0\end{array}$ & $\begin{array}{c}t^{2}+z^{3}+g\left(z, x^{1 / 4}, y^{1 / 3}\right) \subset \mathbb{P}(3,2,3,1) \\
\text { Diff }=\left(0,0, \frac{3}{4}, \frac{2}{3}\right)\end{array}$ & 4 \\
\hline 28 & $\begin{array}{c}x^{8}+a x^{4} y^{n}+b y^{2 n} \\
n=5 \quad b \neq 0 ; 7 ; 11\end{array}$ & $\begin{array}{c}t^{2}+z+x^{2}+a x y+b y^{2} \subset \mathbb{P}(1,2,1,1) \\
\mathbb{P}^{2}, \text { Diff }=\left(0, \frac{2}{3}, \frac{3}{4}, \frac{n-1}{n}\right)\end{array}$ & $6,9,12$ \\
\hline 29 & $\begin{array}{c}a z y^{2} x^{2 i} f_{2-i}\left(x^{2}, y^{3}\right)+ \\
x^{2 j} f_{4-j}\left(x^{2}, y^{3}\right), i \leqslant 1 \| j \leqslant 1\end{array}$ & $\begin{array}{c}t^{2}+z^{3}+g\left(z, x^{1 / 2}, y\right) \subset \mathbb{P}(6,4,3,1) \\
\text { Diff }=\left(0,0, \frac{1}{2}, 0\right)\end{array}$ & 2 \\
\hline 30 & $\begin{array}{c}x^{i} f_{8-i}\left(x, y^{2}\right) \\
i \leqslant 4\end{array}$ & $\begin{array}{c}t^{2}+z+x^{i} f_{8-i}(x, y) \subset \mathbb{P}(4,8,1,1) \\
\mathbb{P}(4,1,1), \text { Diff }=\left(0, \frac{2}{3}, 0, \frac{1}{2}\right)\end{array}$ & 3 \\
\hline 31 & $\begin{array}{c}x^{2 i} f_{4-i}\left(x^{2}, y^{5}\right) \\
i \leqslant 2\end{array}$ & $\begin{array}{c}t^{2}+z+x^{i} f_{4-i}(x, y) \subset \mathbb{P}(2,4,1,1) \\
\mathbb{P}(2,1,1), \text { Diff }=\left(0, \frac{2}{3}, \frac{1}{2}, \frac{4}{5}\right)\end{array}$ & 6 \\
\hline 32 & $\begin{array}{l}x^{8}+x y^{n}, n=8 ; 10 \\
11 ; 13 ; 16,17 ; 19,20\end{array}$ & $\begin{array}{c}t^{2}+z+x^{8}+x y \subset \mathbb{P}(4,8,1,7) \\
\mathbb{P}(4,1,7), \text { Diff }=\left(0, \frac{2}{3}, 0, \frac{n-1}{n}\right)\end{array}$ & $\begin{array}{l}9,10,12 \\
13,18,21\end{array}$ \\
\hline 33 & $\begin{array}{c}x^{8}+a z x^{3} y^{n}+b x y^{3 n} \\
b \neq 0 \&(n=3,4) ; 5,6\end{array}$ & $\begin{array}{c}t^{2}+z^{3}+g\left(z, x, y^{1 / n}\right) \subset \mathbb{P}(12,8,3,7) \\
\text { Diff }=\left(0,0,0, \frac{n-1}{n}\right)\end{array}$ & 4,7 \\
\hline 34 & $\begin{array}{c}x^{8}+x^{2} y^{n} \\
n=7 ; 11 ; 13 ; 17\end{array}$ & $\begin{array}{c}t^{2}+z+x^{4}+x y \subset \mathbb{P}(2,4,1,3) \\
\mathbb{P}(2,1,3), \text { Diff }=\left(0, \frac{2}{3}, \frac{1}{2}, \frac{n-1}{n}\right)\end{array}$ & $\begin{array}{c}7,12,15 \\
18\end{array}$ \\
\hline 35 & $\begin{array}{c}x^{8}+a x^{5} y^{n}+b x^{2} y^{2 n} \\
b \neq 0 \&(n=4,5) ; 7,8\end{array}$ & $\begin{array}{c}t^{2}+z+x^{8}+a x^{5} y+b x^{2} y^{2} \subset \mathbb{P}(4,8,1,3) \\
\mathbb{P}(4,1,3), \text { Diff }=\left(0, \frac{2}{3}, 0, \frac{n-1}{n}\right)\end{array}$ & 6,9 \\
\hline 36 & $\begin{array}{c}\quad x^{8}+x^{3} y^{n} \\
n=7 ; 8 ; 11 ; 13,14\end{array}$ & $\begin{array}{l}t^{2}+z+x^{8}+x^{3} y \subset \mathbb{P}(4,8,1,5) \\
\mathbb{P}(4,1,5), \text { Diff }=\left(0, \frac{2}{3}, 0, \frac{n-1}{n}\right)\end{array}$ & $\begin{array}{c}7,9,12 \\
15\end{array}$ \\
\hline 37 & $\begin{array}{l}x^{8}+a z x^{2} y^{2 n}+ \\
+b x^{3} y^{3 n}, n=3,4\end{array}$ & $\begin{array}{c}t^{2}+z^{3}+g\left(z, x, y^{1 / n}\right) \subset \mathbb{P}(12,8,3,5) \\
\text { Diff }=\left(0,0,0, \frac{n-1}{n}\right)\end{array}$ & 5 \\
\hline 38 & $\begin{aligned} & x^{8}+z y^{n} \\
n= & 7 ; 9 ; 11,13 ; 15\end{aligned}$ & $\begin{array}{c}t^{2}+z^{3}+x^{2}+z y \subset \mathbb{P}(3,2,3,4) \\
\operatorname{Diff}=\left(0,0, \frac{3}{4}, \frac{n-1}{n}\right)\end{array}$ & $\begin{array}{c}7,10,13 \\
16\end{array}$ \\
\hline 39 & $\begin{array}{c}x^{8}+z x y^{n}, n=5 ; 6,7 \\
8,9,10 ; 11,12\end{array}$ & $\begin{array}{c}t^{2}+z^{3}+x^{8}+z x y \subset \mathbb{P}(12,8,3,13) \\
\text { Diff }=\left(0,0,0, \frac{n-1}{n}\right)\end{array}$ & $\begin{array}{c}5,7,10 \\
13\end{array}$ \\
\hline 40 & $\begin{array}{c}x^{8}+z x^{2} y^{n} \\
n=7 ; 9\end{array}$ & $\begin{array}{c}t^{2}+z^{3}+x^{4}+z x y \subset \mathbb{P}(6,4,3,5) \\
\text { Diff }=\left(0,0, \frac{1}{2}, \frac{n-1}{n}\right)\end{array}$ & 7,10 \\
\hline 41 & $\begin{array}{c}x^{7} y+y^{n} \\
n=11 ; 13 ; 17 ; 19 ; 23 \\
25 ; 31 ; 35\end{array}$ & $\begin{array}{l}t+z+x y+y^{n} \subset \mathbb{P}(n, n, n-1,1) \\
\mathbb{P}(n, n-1,1), \text { Diff }=\left(\frac{1}{2}, \frac{2}{3}, \frac{6}{7}, 0\right)\end{array}$ & $\begin{array}{c}12,15,21, \\
22,28,30, \\
36,42\end{array}$ \\
\hline 42 & $\begin{array}{c}x^{7} y+a z y^{4 n}+ \\
+b y^{6 n}, n=2,3,4,5\end{array}$ & $\begin{array}{c}t^{2}+z^{3}+g\left(z, x^{1 / 7}, y\right) \subset \mathbb{P}(3 n, 2 n, 6 n-1,1) \\
\text { Diff }=\left(0,0, \frac{6}{7}, 0\right)\end{array}$ & 7 \\
\hline 43 & $\begin{array}{c}x^{7} y+a z y^{2 n}+ \\
+b y^{3 n}, n=3 ; 7 ; 9,11\end{array}$ & $\begin{array}{l}t+z^{3}+g\left(z, x^{1 / 7}, y\right) \subset \mathbb{P}(3 n, n, 3 n-1,1) \\
\quad \mathbb{P}(n, 3 n-1,1), \text { Diff }=\left(\frac{1}{2}, 0, \frac{6}{7}, 0\right)\end{array}$ & $7,8,14$ \\
\hline 44 & $\begin{array}{l}x^{7} y+y^{2 n}, n=5 ; 7 \\
8 ; 10,13 ; 14,16,17\end{array}$ & $\begin{array}{c}t^{2}+z+x y+y^{2 n} \subset \mathbb{P}(n, 2 n, 2 n-1,1) \\
\mathbb{P}(n, 2 n-1,1), \text { Diff }=\left(0, \frac{2}{3}, \frac{6}{7}, 0\right)\end{array}$ & $\begin{array}{c}7,9,15 \\
21\end{array}$ \\
\hline
\end{tabular}


Продолжение таблицы 1

\begin{tabular}{|c|c|c|c|}
\hline 45 & $\begin{array}{l}\underline{a} z x^{i} f_{5-i}\left(x, y^{2}\right)+ \\
+\underline{b} y x^{j} f_{7-j}\left(x, y^{2}\right) \\
\quad i \leqslant 2 \| j \leqslant 4\end{array}$ & $\begin{array}{l}t+z^{3}+g(z, x, y) \subset \mathbb{P}(15,5,2,1) \\
\mathbb{P}(5,2,1), \text { Diff }=\left(\frac{1}{2}, 0,0,0\right)\end{array}$ & 2 \\
\hline 46 & $\begin{array}{c}y x^{i} f_{7-i}\left(x, y^{3}\right) \\
i \leqslant 5\end{array}$ & $\begin{array}{c}t^{2}+z+g(z, x, y) \subset \mathbb{P}(11,22,3,1) \\
\mathbb{P}(11,3,1), \text { Diff }=\left(0, \frac{2}{3}, 0,0\right)\end{array}$ & 3 \\
\hline 47 & $\begin{array}{c}y x^{i} f_{7-i}\left(x, y^{4}\right) \\
i \leqslant 5\end{array}$ & $\begin{array}{c}t+z+g(z, x, y) \subset \mathbb{P}(29,29,4,1) \\
\mathbb{P}(29,4,1), \text { Diff }=\left(\frac{1}{2}, \frac{2}{3}, 0,0\right)\end{array}$ & 6 \\
\hline 48 & $\begin{array}{c}x^{7} y+x y^{n}, n=8 ; 12 \\
14 ; 18 ; 20 ; 24,26 ; 30\end{array}$ & $\begin{array}{l}t+z+g(z, x, y) \subset \mathbb{P}(7 n-1,7 n-1, n-1,6) \\
\quad \mathbb{P}(7 n-1, n-1,6), \text { Diff }=\left(\frac{1}{2}, \frac{2}{3}, 0,0\right)\end{array}$ & $\begin{array}{l}9,15,16 \\
22,24,30 \\
36\end{array}$ \\
\hline 49 & $\begin{array}{c}x^{7} y+a x^{4} y^{n+1}+b x y^{2 n+1} \\
n=4 b \neq 0 ; 8,10 ; 14\end{array}$ & $\begin{array}{c}t+z+g(z, x, y) \subset \mathbb{P}(7 n+3,7 n+3, n, 3) \\
\mathbb{P}(7 n+3, n, 3), \text { Diff }=\left(\frac{1}{2}, \frac{2}{3}, 0,0\right)\end{array}$ & $6,12,18$ \\
\hline 50 & $\begin{array}{c}x^{7} y+a x^{4} y^{2 n+2}+b x y^{4 n+3} \\
n=2 b \neq 0 ; 3,5,6\end{array}$ & $\begin{array}{c}t^{2}+z+g(z, x, y) \subset \mathbb{P}(7 n+5,14 n+10,2 n+1,3) \\
\mathbb{P}(7 n+5,2 n+1,3), \text { Diff }=\left(0, \frac{2}{3}, 0,0\right)\end{array}$ & 3,9 \\
\hline 51 & $\begin{array}{c}y x^{2 i+1} f_{3-i}\left(x^{2}, y^{n}\right) \\
(n=3,5) \& i \leqslant 1 ; 9\end{array}$ & $\begin{array}{c}t+z+g(z, x, y) \subset \mathbb{P}(7 n+2,7 n+2, n, 2) \\
\mathbb{P}(7 n+2, n, 2), \text { Diff }=\left(\frac{1}{2}, \frac{2}{3}, 0,0\right)\end{array}$ & 6,12 \\
\hline 52 & $\begin{array}{l}a z y^{3} x^{2 i} f_{2-i}\left(x^{2}, y^{7}\right)+ \\
+y x^{2 j+1} f_{3-j}\left(x^{2}, y^{7}\right)\end{array}$ & $\begin{array}{c}t+z^{3}+g(z, x, y) \subset \mathbb{P}(51,17,7,2) \\
\mathbb{P}(17,7,2), \text { Diff }=\left(\frac{1}{2}, 0,0,0\right)\end{array}$ & 4 \\
\hline 53 & $\begin{aligned} & x^{7} y+x^{2} y^{n} \\
n= & 7 ; 9 ; 13 ; 15 ; 19 ; 25\end{aligned}$ & $\begin{array}{c}t+z+g(z, x, y) \subset \mathbb{P}(7 n-2,7 n-2, n-1,5) \\
\mathbb{P}(7 n-2, n-1,5), \text { Diff }=\left(\frac{1}{2}, \frac{2}{3}, 0,0\right)\end{array}$ & $\begin{array}{l}9,10,16 \\
18,24,30\end{array}$ \\
\hline 54 & $\begin{array}{l}x^{7} y+x^{2} y^{2 n} \\
n=5,6 ; 9,11,12\end{array}$ & $\begin{array}{c}t^{2}+z+g(z, x, y) \subset \mathbb{P}(7 n-1,14 n-2,2 n-1,5) \\
\mathbb{P}(7 n-1,2 n-1,5), \text { Diff }=\left(0, \frac{2}{3}, 0,0\right)\end{array}$ & 9,15 \\
\hline 55 & $\begin{array}{l}x^{7} y+a z x^{3} y^{2 n+2}+ \\
+b x^{2} y^{6 n+5}, n=2,3\end{array}$ & $\begin{array}{c}t+z^{3}+g \subset \mathbb{P}(42 n+33,14 n+11,6 n+4,5) \\
\mathbb{P}(14 n+11,6 n+4,5), \text { Diff }=\left(\frac{1}{2}, 0,0,0\right)\end{array}$ & 10 \\
\hline 56 & $\begin{array}{c}x^{7} y+a z x^{3} y^{2 n+1}+ \\
+b x^{2} y^{6 n+2}, n=1 b \neq 0 ; 2,3\end{array}$ & $\begin{array}{c}t^{2}+z^{3}+g \subset \mathbb{P}(21 n+6,14 n+4,6 n+1,5) \\
\text { Diff }=\varnothing\end{array}$ & 4,5 \\
\hline 57 & $\begin{array}{c}\quad x^{7} y+x^{3} y^{n} \\
n=8 ; 10 ; 14,16 ; 20\end{array}$ & $\begin{array}{c}t+z+g(z, x, y) \subset \mathbb{P}(7 n-3,7 n-3, n-1,4) \\
\mathbb{P}(7 n-3, n-1,4), \text { Diff }=\left(\frac{1}{2}, \frac{2}{3}, 0,0\right)\end{array}$ & $\begin{array}{c}10,12,18 \\
24\end{array}$ \\
\hline 58 & $\begin{array}{l}x^{7} y+a z x^{2} y^{2 n}+ \\
+b x^{3} y^{3 n}, n=4,6\end{array}$ & $\begin{array}{c}t+z^{3}+g(z, x, y) \subset \mathbb{P}(21 n-3,7 n-1,3 n-1,4) \\
\quad \mathbb{P}(7 n-1,3 n-1,4), \text { Diff }=\left(\frac{1}{2}, 0,0,0\right)\end{array}$ & 8 \\
\hline 59 & $\begin{array}{l}x^{7} y+z y^{n}, n=7 ; 9 \\
11 ; 13 ; 15,19 ; 21,23\end{array}$ & $\begin{array}{c}t+z^{3}+x y+z y^{n} \subset \mathbb{P}(3 n, n, 3 n-2,2) \\
\mathbb{P}(n, 3 n-2,2), \text { Diff }=\left(\frac{1}{2}, 0, \frac{6}{7}, 0\right)\end{array}$ & $\begin{array}{l}8,10,14 \\
16,22,28\end{array}$ \\
\hline 60 & $\begin{array}{c}\quad x^{7} y+z x y^{n} \\
n=6 ; 10,12,14 ; 16,18\end{array}$ & $\begin{array}{c}t+z^{3}+g(z, x, y) \subset \mathbb{P}(21 n-3,7 n-1,3 n-2,11) \\
\mathbb{P}(7 n-1,3 n-2,11), \text { Diff }=\left(\frac{1}{2}, 0,0,0\right)\end{array}$ & $8,16,22$ \\
\hline 61 & $\begin{array}{c}x^{7} y+z x y^{2 n+1} \\
n=2,3,4 ; 5,6,7,8\end{array}$ & $\begin{array}{c}t^{2}+z^{3}+g \subset \mathbb{P}(21 n+9,14 n+6,6 n+1,11) \\
\text { Diff }=\varnothing\end{array}$ & 5,11 \\
\hline 62 & $\begin{array}{c}x^{7} y+z x^{2} y^{n} \\
n=5,7,9 ; 11,13\end{array}$ & $\begin{array}{c}t+z^{3}+g(z, x, y) \subset \mathbb{P}(21 n-6,7 n-2,3 n-2,8) \\
\quad \mathbb{P}(7 n-2,3 n-2,8), \text { Diff }=\left(\frac{1}{2}, 0,0,0\right)\end{array}$ & 10,16 \\
\hline
\end{tabular}


Продолжение таблицы 1

\begin{tabular}{|c|c|c|c|}
\hline 63 & $\begin{array}{c}\underline{a} z x^{i} y^{i_{1}} f_{6-i-i_{1}}(x, y)+ \\
\quad+\underline{b} x^{j} y^{j_{1}} f_{9-j-j_{1}}(x, y) \\
(i=1|| j \leqslant 2) \&\left(i_{1}=1|| j_{1} \leqslant 2\right)\end{array}$ & $\begin{array}{c}t+z^{3}+g(z, x, y) \subset \mathbb{P}(9,3,1,1) \\
\mathbb{P}(3,1,1), \text { Diff }=\left(\frac{1}{2}, 0,0,0\right)\end{array}$ & 2 \\
\hline 64 & $\begin{array}{c}a x^{9}+b z x^{6}+y^{2 n} \\
n=5 ; 7 ; 8\end{array}$ & $\begin{array}{c}t^{2}+z^{3}+a x^{3}+b z x^{2}+y^{2} \subset \mathbb{P}(3,2,2,3) \\
\text { Diff }=\left(0,0, \frac{2}{3}, \frac{n-1}{n}\right)\end{array}$ & $5,7,9$ \\
\hline 65 & $\begin{array}{c}a x^{9}+b z x^{6}+y^{n} \\
n=11 ; 13 ; 17\end{array}$ & $\begin{array}{c}t+z^{3}+a x^{3}+y+b z x^{2} \subset \mathbb{P}(3,1,1,3) \\
\mathbb{P}(1,1,3), \text { Diff }=\left(\frac{1}{2}, 0, \frac{2}{3}, \frac{n-1}{n}\right)\end{array}$ & $12,14,18$ \\
\hline 66 & $\begin{array}{c}\underline{a} z x^{3 i} f_{2-i}\left(x^{3}, y^{4}\right)+ \\
+\underline{b} x^{3 j} f_{3-j}\left(x^{3}, y^{4}\right), i=0|| j \leqslant 1\end{array}$ & $\begin{array}{c}t^{2}+z^{3}+g\left(z, x^{1 / 3}, y^{1 / 2}\right) \subset \mathbb{P}(3,2,2,1) \\
\text { Diff }=\left(0,0, \frac{2}{3}, \frac{1}{2}\right)\end{array}$ & 3 \\
\hline 67 & $\begin{array}{c}\underline{a} z x^{3 i} f_{2-i}\left(x^{3}, y^{5}\right)+ \\
+\underline{b} x^{3 j} f_{3-j}\left(x^{3}, y^{5}\right), i \leqslant 1|| j \leqslant 1 \\
\end{array}$ & $\begin{array}{c}t+z^{3}+g\left(z, x^{1 / 3}, y^{1 / 5}\right) \subset \mathbb{P}(3,1,1,1) \\
\mathbb{P}^{2}, \text { Diff }=\left(\frac{1}{2}, 0, \frac{2}{3}, \frac{4}{5}\right)\end{array}$ & 6 \\
\hline 68 & $\begin{array}{c}a x^{9}+b z x^{6}+x y^{n} \\
n=9 ; 11 ; 13 ; 15\end{array}$ & $\begin{array}{c}t+z^{3}+a x^{9}+x y+b z x^{6} \subset \mathbb{P}(9,3,1,8) \\
\mathbb{P}(3,1,8), \text { Diff }=\left(\frac{1}{2}, 0,0, \frac{n-1}{n}\right)\end{array}$ & $\begin{array}{c}10,12,14, \\
16\end{array}$ \\
\hline 69 & $\begin{array}{c}\underline{a} z x^{2+4 i} f_{1-i}\left(x^{4}, y^{n}\right)+ \\
+\underline{b} x^{4 j+1} f_{2-j}\left(x^{4}, y^{n}\right) \\
\quad n=5 i=0 \| j=0 ; 7\end{array}$ & $\begin{array}{c}t+z^{3}+g\left(z, x, y^{1 / n}\right) \subset \mathbb{P}(9,3,1,4) \\
\mathbb{P}(3,1,4), \text { Diff }=\left(\frac{1}{2}, 0,0, \frac{n-1}{n}\right)\end{array}$ & 6,8 \\
\hline 70 & $\begin{array}{c}\underline{a} z x^{2 i} f_{3-i}\left(x^{2}, y^{3}\right)+ \\
\underline{b} x^{2 j+1} f_{4-j}\left(x^{2}, y^{3}\right) \\
i \leqslant 1|| j \leqslant 1\end{array}$ & $\begin{array}{c}t+z^{3}+g\left(z, x, y^{1 / 3}\right) \subset \mathbb{P}(9,3,1,2) \\
\mathbb{P}(3,1,2), \text { Diff }=\left(\frac{1}{2}, 0,0, \frac{2}{3}\right)\end{array}$ & 4 \\
\hline 71 & $\begin{array}{c}a x^{9}+b z x^{6}+x^{2} y^{2 n} \\
n=4,5 ; 6\end{array}$ & $\begin{array}{c}t^{2}+z^{3}+g\left(z, x, y^{1 / n}\right) \subset \mathbb{P}(9,6,2,7) \\
\text { Diff }=\left(0,0,0, \frac{n-1}{n}\right)\end{array}$ & 5,7 \\
\hline 72 & $\begin{array}{c}a x^{9}+b z x^{6}+x^{2} y^{n} \\
n=9 ; 11 ; 13\end{array}$ & $\begin{array}{c}t+z^{3}+g\left(z, x, y^{1 / n}\right) \subset \mathbb{P}(9,3,1,7) \\
\mathbb{P}(3,1,7), \text { Diff }=\left(\frac{1}{2}, 0,0, \frac{n-1}{n}\right)\end{array}$ & $10,12,14$ \\
\hline 73 & $\begin{array}{c}\underline{a} z x^{6 i} f_{1-i}\left(x^{6}, y^{n}\right)+ \\
\underline{b} x^{6 j+3} f_{1-j}\left(x^{6}, y^{n}\right), n=7 ; 11\end{array}$ & $\begin{array}{l}t+z^{3}+g\left(z, x^{1 / 3}, y^{1 / n}\right) \subset \mathbb{P}(3,1,1,2) \\
\quad \mathbb{P}(1,1,2), \text { Diff }=\left(\frac{1}{2}, 0, \frac{2}{3}, \frac{n-1}{n}\right)\end{array}$ & 8,12 \\
\hline 74 & $\begin{array}{c}d x^{9}+a z x y^{2 n}+b x^{4} y^{2 n}+ \\
+c z x^{6} \\
(n=3 a \neq 0 ; 4) \&|d|+|c| \neq 0\end{array}$ & $\begin{array}{c}t^{2}+z^{3}+g\left(z, x, y^{1 / n}\right) \subset \mathbb{P}(9,6,2,5) \\
\text { Diff }=\left(0,0,0, \frac{n-1}{n}\right)\end{array}$ & 3,5 \\
\hline 75 & $\begin{array}{c}d x^{9}+a z x y^{n}+b x^{4} y^{n}+c z x^{6}, \\
(n=7 ; 9) \& \\
(|a|+|b| \neq 0|d|+|c| \neq 0)\end{array}$ & $\begin{array}{l}t+z^{3}+g\left(z, x, y^{1 / n}\right) \subset \mathbb{P}(9,3,1,5) \\
\mathbb{P}(3,1,5), \text { Diff }=\left(\frac{1}{2}, 0,0, \frac{n-1}{n}\right)\end{array}$ & 8,10 \\
\hline 76 & $\begin{array}{c}x^{8} y+y^{2 n} \\
n=5,8\end{array}$ & $\begin{array}{c}t^{2}+z+x y+y^{2 n} \subset \mathbb{P}(n, 2 n, 2 n-1,1) \\
\mathbb{P}(n, 2 n-1,1), \text { Diff }=\left(0, \frac{2}{3}, \frac{7}{8}, 0\right)\end{array}$ & 9 \\
\hline 77 & $\begin{array}{c}x^{8} y+a z y^{4 n}+b y^{6 n} \\
n=2,3\end{array}$ & $\begin{array}{c}t^{2}+z^{3}+g\left(z, x^{1 / 8}, y\right) \subset \mathbb{P}(3 n, 2 n, 6 n-1,1) \\
\text { Diff }=\left(0,0, \frac{7}{8}, 0\right)\end{array}$ & 8 \\
\hline 78 & $\begin{array}{c}x^{8} y+y^{2 n} \\
n=7 ; 10\end{array}$ & $\begin{array}{c}t^{2}+z+x y+y^{2 n} \subset \mathbb{P}(n, 2 n, 2 n-1,1) \\
\mathbb{P}(n, 2 n-1,1), \text { Diff }=\left(0, \frac{2}{3}, \frac{7}{8}, 0\right)\end{array}$ & 9,24 \\
\hline
\end{tabular}


Продолжение таблицы 1

\begin{tabular}{|c|c|c|c|}
\hline 79 & $\begin{array}{l}y x^{4 i} f_{2-i}\left(x^{4}, y^{n-1}\right) \\
n=6 i=0 ; n=10 i \leqslant 1\end{array}$ & $\begin{array}{c}t^{2}+z+g\left(z, x^{1 / 2}, y\right) \subset \mathbb{P}(2 n-1,4 n-2, n-1,2) \\
\mathbb{P}(2 n-1, n-1,2), \text { Diff }=\left(0, \frac{2}{3}, \frac{1}{2}, 0\right)\end{array}$ & 3,12 \\
\hline 80 & $\begin{array}{c}a z y^{3} x^{4 i} f_{1-i}\left(x^{4}, y^{7}\right)+ \\
+y x^{4 j} f_{2-j}\left(x^{4}, y^{7}\right) \\
i=0 \| j \leqslant 1\end{array}$ & $\begin{array}{c}t^{2}+z^{3}+g\left(z, x^{1 / 2}, y\right) \subset \mathbb{P}(15,10,7,2) \\
\text { Diff }=\left(0,0, \frac{1}{2}, 0\right)\end{array}$ & 4 \\
\hline 81 & $\begin{array}{c}y x^{2 i} f_{4-i}\left(x^{2}, y^{3}\right) \\
i \leqslant 2\end{array}$ & $\begin{array}{c}t^{2}+z+g(z, x, y) \subset \mathbb{P}(13,26,3,2) \\
\mathbb{P}(13,3,2), \text { Diff }=\left(0, \frac{2}{3}, 0,0\right)\end{array}$ & 3 \\
\hline 82 & $\begin{array}{c}y x^{i} f_{8-i}\left(x, y^{2}\right) \\
i \leqslant 5\end{array}$ & $\begin{array}{c}t+z+g(z, x, y) \subset \mathbb{P}(17,17,2,1) \\
\mathbb{P}(17,2,1), \text { Diff }=\left(\frac{1}{2}, \frac{2}{3}, 0,0\right)\end{array}$ & 6 \\
\hline 83 & $\begin{array}{c}\quad x^{8} y+x y^{n} \\
n=9 ; 10 ; 12,16 ; 13 ; 18\end{array}$ & $\begin{array}{l}t+z+g(z, x, y) \subset \mathbb{P}(8 n-1,8 n-1, n-1,7) \\
\quad \mathbb{P}(8 n-1, n-1,7), \text { Diff }=\left(\frac{1}{2}, \frac{2}{3}, 0,0\right)\end{array}$ & $\begin{array}{c}10,12,18 \\
30,42\end{array}$ \\
\hline 84 & $\begin{array}{c}x^{8} y+a z x^{3} y^{n+1}+b x y^{3 n+2} \\
n=3 \quad b \neq 0 ; 4,5\end{array}$ & $\begin{array}{c}t+z^{3}+g(z, x, y) \subset \mathbb{P}(24 n+15,8 n+5,3 n+1,7) \\
\mathbb{P}(8 n+5,3 n+1,7), \text { Diff }=\left(\frac{1}{2}, 0,0,0\right)\end{array}$ & 4,14 \\
\hline 85 & $\begin{array}{l}x^{8} y+x^{2} y^{n} \\
n=8,12,14\end{array}$ & $\begin{array}{c}t^{2}+z+g(z, x, y) \subset \mathbb{P}(4 n-1,8 n-2, n-1,6) \\
\mathbb{P}(4 n-1, n-1,6), \text { Diff }=\left(0, \frac{2}{3}, 0,0\right)\end{array}$ & 9 \\
\hline 86 & $\begin{array}{c}x^{8} y+a x^{5} y^{n+1}+b x^{2} y^{2 n+1} \\
(n=4,5) \& b \neq 0 ; 7\end{array}$ & $\begin{array}{l}t+z+g(z, x, y) \subset \mathbb{P}(8 n+3,8 n+3, n, 3) \\
\mathbb{P}(8 n+3, n, 3), \text { Diff }=\left(\frac{1}{2}, \frac{2}{3}, 0,0\right)\end{array}$ & 6,18 \\
\hline 87 & $\begin{array}{l}x^{8} y+x^{3} y^{n} \\
n=7,8,10 ; 13\end{array}$ & $\begin{array}{c}t+z+g(z, x, y) \subset \mathbb{P}(8 n-3,8 n-3, n-1,5) \\
\mathbb{P}(8 n-3, n-1,5), \text { Diff }=\left(\frac{1}{2}, \frac{2}{3}, 0,0\right)\end{array}$ & 12,30 \\
\hline 88 & $\begin{array}{c}x^{8} y+a z x^{2} y^{2 n}+b x^{3} y^{3 n} \\
n=3,4\end{array}$ & $\begin{array}{c}t+z^{3}+g(z, x, y) \subset \mathbb{P}(24 n-3,8 n-1,3 n-1,5) \\
\quad \mathbb{P}(8 n-1,3 n-1,5), \text { Diff }=\left(\frac{1}{2}, 0,0,0\right)\end{array}$ & 10 \\
\hline 89 & $\begin{array}{c}x^{8} y+z y^{n} \\
n=7,9 ; 11,13\end{array}$ & $\begin{array}{c}t^{2}+z^{3}+x^{2} y+z y^{n} \subset \mathbb{P}(3 n, 2 n, 3 n-2,4) \\
\text { Diff }=\left(0,0, \frac{3}{4}, 0\right)\end{array}$ & 5,16 \\
\hline 90 & $\begin{array}{c}\quad x^{8} y+z x y^{n} \\
n=6,7,8,9 ; 10,11\end{array}$ & $\begin{array}{c}t+z^{3}+g(z, x, y) \subset \mathbb{P}(24 n-3,8 n-1,3 n-2,13) \\
\mathbb{P}(8 n-1,3 n-2,13), \text { Diff }=\left(\frac{1}{2}, 0,0,0\right)\end{array}$ & 10,26 \\
\hline 91 & $\begin{array}{c}x^{8} y+z x^{2} y^{n} \\
n=5,7\end{array}$ & $\begin{array}{c}t^{2}+z^{3}+g(z, x, y) \subset \mathbb{P}(12 n-3,8 n-2,3 n-2,10) \\
\text { Diff }=\varnothing\end{array}$ & 10 \\
\hline 92 & $\begin{array}{c}x^{7} y^{2}+y^{n} \\
n=11 ; 13 ; 17 ; 19 ; 25 ; 29\end{array}$ & $\begin{array}{l}t+z+x y^{2}+y^{n} \subset \mathbb{P}(n, n, n-2,1) \\
\mathbb{P}(n, n-2,1), \text { Diff }=\left(\frac{1}{2}, \frac{2}{3}, \frac{6}{7}, 0\right)\end{array}$ & $\begin{array}{l}15,18,24, \\
28,36,42\end{array}$ \\
\hline 93 & $\begin{array}{c}x^{7} y^{2}+y^{2 n} \\
n=5 ; 7 ; 11 ; 13\end{array}$ & $\begin{array}{c}t^{2}+z+x y^{2}+y^{2 n} \subset \mathbb{P}(n, 2 n, 2 n-2,1) \\
\mathbb{P}(n, 2 n-2,1), \text { Diff }=\left(0, \frac{2}{3}, \frac{6}{7}, 0\right)\end{array}$ & $\begin{array}{c}7,9,15, \\
21\end{array}$ \\
\hline 94 & $\begin{array}{c}x^{7} y^{2}+y^{4 n} \\
n=5 ; 7\end{array}$ & $\begin{array}{l}t^{2}+z+x y^{2}+y^{2 n} \subset \mathbb{P}(n, 2 n, 2 n-1,1) \\
\quad \mathbb{P}(n, 2 n-1,1), \text { Diff }=\left(0, \frac{2}{3}, \frac{6}{7}, \frac{1}{2}\right)\end{array}$ & 15,21 \\
\hline 95 & $\begin{array}{c}x^{7} y^{2}+a z y^{2 n}+b y^{3 n} \\
n=5 ; 7,9\end{array}$ & $\begin{array}{c}t+z^{3}+g\left(z, x^{1 / 7}, y\right) \subset \mathbb{P}(3 n, n, 3 n-2,1) \\
\mathbb{P}(n, 3 n-2,1), \text { Diff }=\left(\frac{1}{2}, 0, \frac{6}{7}, 0\right)\end{array}$ & 8,14 \\
\hline 96 & $\begin{array}{c}x^{7} y^{2}+a z y^{8 n}+b y^{12 n} \\
n=1,2\end{array}$ & $\begin{array}{c}t^{2}+z^{3}+g\left(z, x^{1 / 7}, y^{1 / 2}\right) \subset \mathbb{P}(3 n, 2 n, 6 n-1,1) \\
\text { Diff }=\left(0,0, \frac{6}{7}, \frac{1}{2}\right)\end{array}$ & 7 \\
\hline
\end{tabular}


Продолжение таблицы 1

\begin{tabular}{|c|c|c|c|}
\hline 97 & $x^{7} y^{2}+a z y^{12}+b y^{18}$ & $\begin{array}{c}t^{2}+z^{3}+g\left(z, x^{1 / 7}, y\right) \subset \mathbb{P}(9,6,16,1) \\
\text { Diff }=\left(0,0, \frac{6}{7}, 0\right)\end{array}$ & 7 \\
\hline 98 & $\begin{array}{c}y^{2} x^{i} f_{7-i}\left(x, y^{2}\right) \\
i \leqslant 4\end{array}$ & $\begin{array}{c}t^{2}+z+y x^{i} f_{7-i}(x, y) \subset \mathbb{P}(4,8,1,1) \\
\mathbb{P}(4,1,1), \text { Diff }=\left(0, \frac{2}{3}, 0, \frac{1}{2}\right)\end{array}$ & 3 \\
\hline 99 & $\begin{array}{c}y^{2} x^{i} f_{7-i}\left(x, y^{3}\right) \\
i \leqslant 5\end{array}$ & $\begin{array}{c}t+z+g(z, x, y) \subset \mathbb{P}(23,23,3,1) \\
\mathbb{P}(23,3,1), \text { Diff }=\left(\frac{1}{2}, \frac{2}{3}, 0,0\right)\end{array}$ & 6 \\
\hline 100 & $\begin{array}{c}\quad x^{7} y^{2}+x y^{n} \\
n=9 ; 13 ; 15 ; 19,21 ; 25\end{array}$ & $\begin{array}{l}t+z+g(z, x, y) \subset \mathbb{P}(7 n-2,7 n-2, n-2,6) \\
\mathbb{P}(7 n-2, n-2,6), \text { Diff }=\left(\frac{1}{2}, \frac{2}{3}, 0,0\right)\end{array}$ & $\begin{array}{c}12,18,22, \\
30,36\end{array}$ \\
\hline 101 & $\begin{array}{c}x^{3 i+1} y^{2} f_{2-i}\left(x^{3}, y^{2 n}\right) \\
n=2 i=0 ; 4 i \leqslant 1\end{array}$ & $\begin{array}{c}t^{2}+z+g(z, x, y) \subset \mathbb{P}(7 n+3,14 n+6,2 n, 3) \\
\mathbb{P}(7 n+3,2 n, 3), \text { Diff }=\left(0, \frac{2}{3}, 0,0\right)\end{array}$ & 3,9 \\
\hline 102 & $x^{7} y^{2}+a x^{4} y^{12}+b x y^{22}$ & $\begin{array}{c}t^{2}+z+x^{7} y+a x^{4} y^{6}+b x y^{11} \subset \mathbb{P}(19,38,5,3) \\
\mathbb{P}(19,5,3), \text { Diff }=\left(0, \frac{2}{3}, 0, \frac{1}{2}\right)\end{array}$ & 9 \\
\hline 103 & $\begin{array}{c}x^{7} y^{2}+a x^{4} y^{n+1}+b x y^{2 n} \\
n=6,8 ; 12\end{array}$ & $\begin{array}{c}t+z+g(z, x, y) \subset \mathbb{P}(7 n-1,7 n-1, n-1,3) \\
\mathbb{P}(7 n-1, n-1,3), \text { Diff }=\left(\frac{1}{2}, \frac{2}{3}, 0,0\right)\end{array}$ & 12,18 \\
\hline 104 & $\begin{array}{c}y^{2} x^{2 i+1} f_{3-i}\left(x^{2}, y^{n}\right) \\
n=3 i \leqslant 1 ; 7 i \leqslant 2\end{array}$ & $\begin{array}{c}t+z+g(z, x, y) \subset \mathbb{P}(7 n+4,7 n+4, n, 2) \\
\mathbb{P}(7 n+4, n, 2), \mathrm{Diff}=\left(\frac{1}{2}, \frac{2}{3}, 0,0\right)\end{array}$ & 6,12 \\
\hline 105 & $\begin{array}{l}a z y^{3} x^{2 i} f_{2-i}\left(x^{2}, y^{5}\right)+ \\
+y^{2} x^{2 j+1} f_{3-j}\left(x^{2}, y^{5}\right)\end{array}$ & $\begin{array}{c}t+z^{3}+g(z, x, y) \subset \mathbb{P}(39,13,5,2) \\
\mathbb{P}(13,5,2), \text { Diff }=\left(\frac{1}{2}, 0,0,0\right)\end{array}$ & 4 \\
\hline 106 & $\begin{array}{c}x^{7} y^{2}+x^{2} y^{n} \\
n=9 ; 11 ; 15 ; 21\end{array}$ & $\begin{array}{c}t+z+g(z, x, y) \subset \mathbb{P}(7 n-4,7 n-4, n-2,5) \\
\mathbb{P}(7 n-2, n-2,5), \text { Diff }=\left(\frac{1}{2}, \frac{2}{3}, 0,0\right)\end{array}$ & $\begin{array}{c}12,16,24, \\
30\end{array}$ \\
\hline 107 & $\begin{array}{c}x^{7} y^{2}+x^{2} y^{4 n} \\
n=2 ; 5\end{array}$ & $\begin{array}{c}t^{2}+z+x^{7} y+x^{2} y^{2 n} \subset \mathbb{P}(7 n-1,14 n-2,2 n-1,5) \\
\mathbb{P}(7 n-1,2 n-1,5), \text { Diff }=\left(0, \frac{2}{3}, 0, \frac{1}{2}\right)\end{array}$ & 9,15 \\
\hline 108 & $\begin{array}{c}x^{7} y^{2}+x^{2} y^{2 n} \\
n=7 ; 9\end{array}$ & $\begin{array}{l}t^{2}+z+g(z, x, y) \subset \mathbb{P}(7 n-2,14 n-4,2 n-2,5) \\
\quad \mathbb{P}(7 n-2,2 n-2,5), \text { Diff }=\left(0, \frac{2}{3}, 0,0\right)\end{array}$ & 9,15 \\
\hline 109 & $\begin{array}{l}x^{7} y^{2}+a z x^{3} y^{n+1}+ \\
+b x^{2} y^{3 n+1}, n=4,6\end{array}$ & $\begin{array}{c}t+z^{3}+g(z, x, y) \subset \mathbb{P}(21 n+3,7 n+1,3 n-1,5) \\
\mathbb{P}(7 n+1,3 n-1,5), \text { Diff }=\left(\frac{1}{2}, 0,0,0\right)\end{array}$ & 10 \\
\hline 110 & $\begin{array}{c}x^{7} y^{2}+a z x^{3} y^{4}+ \\
+x^{2} y^{10}\end{array}$ & $\begin{array}{c}t^{2}+z^{3}+g(z, x, y) \subset \mathbb{P}(33,22,8,5) \\
\text { Diff }=\varnothing\end{array}$ & 4 \\
\hline 111 & $\begin{array}{c}x^{7} y^{2}+a z x^{3} y^{6} \\
+b x^{2} y^{16}\end{array}$ & $\begin{array}{c}t^{2}+z^{3}+g\left(z, x, y^{1 / 2}\right) \subset \mathbb{P}(27,18,7,5) \\
\text { Diff }=\left(0,0,0, \frac{1}{2}\right)\end{array}$ & 5 \\
\hline 112 & $\begin{array}{l}x^{7} y^{2}+x^{3} y^{n} \\
n=7 ; 11,13 ; 17\end{array}$ & $\begin{array}{l}t+z+g(z, x, y) \subset \mathbb{P}(7 n-6,7 n-6, n-2,4) \\
\quad \mathbb{P}(7 n-6, n-2,4), \text { Diff }=\left(\frac{1}{2}, \frac{2}{3}, 0,0\right)\end{array}$ & $10,18,24$ \\
\hline 113 & $\begin{array}{l}x^{7} y^{2}+a z x^{2} y^{2 n}+ \\
+b x^{3} y^{3 n}, n=3,5\end{array}$ & $\begin{array}{c}t+z^{3}+g(z, x, y) \subset \mathbb{P}(21 n-6,7 n-2,3 n-2,4) \\
\mathbb{P}(7 n-2,3 n-2,4), \text { Diff }=\left(\frac{1}{2}, 0,0,0\right)\end{array}$ & 8 \\
\hline 114 & $\begin{aligned} & x^{7} y^{2}+z y^{n} \\
n= & 7 ; 9 ; 11 ; 15 ; 17,19\end{aligned}$ & $\begin{array}{c}t+z^{3}+x y^{2}+z y^{n} \subset \mathbb{P}(3 n, n, 3 n-4,2) \\
\mathbb{P}(n, 3 n-4,2), \text { Diff }=\left(\frac{1}{2}, 0, \frac{6}{7}, 0\right)\end{array}$ & $\begin{array}{c}10,14,16, \\
22,28\end{array}$ \\
\hline 115 & $\begin{array}{c}x^{7} y^{2}+z x y^{4 n+2} \\
n=1 ; 2,3\end{array}$ & $\begin{array}{c}t^{2}+z^{3}+g\left(z, x, y^{1 / 2}\right) \subset \mathbb{P}(21 n+9,14 n+6,6 n+1,11) \\
\text { Diff }=\left(0,0,0, \frac{1}{2}\right)\end{array}$ & 5,11 \\
\hline
\end{tabular}


Продолжение таблицы 1

\begin{tabular}{|c|c|c|c|}
\hline 116 & $\begin{array}{l}x^{7} y^{2}+z x y^{n} \\
n=7 ; 9,11 ; 13,15\end{array}$ & $\begin{array}{c}t+z^{3}+g(z, x, y) \subset \mathbb{P}(21 n-6,7 n-2,3 n-4,11) \\
\mathbb{P}(7 n-2,3 n-4,11), \text { Diff }=\left(\frac{1}{2}, 0,0,0\right)\end{array}$ & $10,16,22$ \\
\hline 117 & $\begin{array}{c}x^{7} y^{2}+z x y^{4 n} \\
n=2 ; 3\end{array}$ & $\begin{array}{c}t^{2}+z^{3}+g(z, x, y) \subset \mathbb{P}(42 n-3,28 n-2,12 n-4,11) \\
\text { Diff }=\varnothing\end{array}$ & 5,11 \\
\hline 118 & $\begin{array}{l}x^{7} y^{2}+z x^{2} y^{n} \\
n=5,7 ; 9,11\end{array}$ & $\begin{array}{c}t+z^{3}+g(z, x, y) \subset \mathbb{P}(21 n-12,7 n-4,3 n-4,8) \\
\mathbb{P}(7 n-4,3 n-4,8), \text { Diff }=\left(\frac{1}{2}, 0,0,0\right)\end{array}$ & 10,16 \\
\hline 119 & $\begin{array}{c}x^{i} y^{j} f_{10-i-j}(x, y) \\
i \leqslant 3 \& j \leqslant 3\end{array}$ & $\begin{array}{c}t^{2}+z+g(z, x, y) \subset \mathbb{P}(5,10,1,1) \\
\mathbb{P}(5,1,1), \text { Diff }=\left(0, \frac{2}{3}, 0,0\right)\end{array}$ & 3 \\
\hline 120 & $\begin{array}{l}x^{10}+y^{n} \\
n=11 ; 13\end{array}$ & $\begin{array}{c}t^{2}+z+x^{2}+y \subset \mathbb{P}(1,2,1,2) \\
\mathbb{P}(1,1,2), \text { Diff }=\left(0, \frac{2}{3}, \frac{4}{5}, \frac{n-1}{n}\right)\end{array}$ & 12,15 \\
\hline 121 & $\begin{array}{c}z y^{2}\left(\underline{a} x^{5}+b y^{6}\right)+ \\
\underline{c} x^{5 j} f_{2-j}\left(x^{5}, y^{6}\right), b \neq 0 \| j=0\end{array}$ & $\begin{array}{c}t^{2}+z^{3}+g\left(z, x^{1 / 5}, y^{1 / 2}\right) \subset \mathbb{P}(3,2,3,1) \\
\text { Diff }=\left(0,0, \frac{4}{5}, \frac{1}{2}\right)\end{array}$ & 5 \\
\hline 122 & $x^{10}+a x^{5} y^{7}+b y^{14}$ & $\begin{array}{c}t^{2}+z+x^{2}+a x y+b y^{2} \subset \mathbb{P}(1,2,1,1) \\
\mathbb{P}^{2}, \text { Diff }=\left(0, \frac{2}{3}, \frac{4}{5}, \frac{6}{7}\right)\end{array}$ & 15 \\
\hline 123 & $\begin{array}{c}x^{10}+x y^{n} \\
n=10,11 ; 13\end{array}$ & $\begin{array}{l}t^{2}+z+x^{10}+x y \subset \mathbb{P}(5,10,1,9) \\
\mathbb{P}(5,1,9), \text { Diff }=\left(0, \frac{2}{3}, 0, \frac{n-1}{n}\right)\end{array}$ & 12,27 \\
\hline 124 & $\begin{array}{c}x^{3 i+1} f_{3-i}\left(x^{3}, y^{4}\right) \\
i=0,1\end{array}$ & $\begin{array}{c}t^{2}+z+x^{3 i+1} f_{3-i}\left(x^{3}, y\right) \subset \mathbb{P}(5,10,1,3) \\
\mathbb{P}(5,1,3), \text { Diff }=\left(0, \frac{2}{3}, 0, \frac{3}{4}\right)\end{array}$ & 9 \\
\hline 125 & $\begin{array}{c}x^{10}+a z x^{4} y^{3}+ \\
+x^{2} y^{9}\end{array}$ & $\begin{array}{c}t^{2}+z^{3}+x^{10}+a z x^{4} y+x^{2} y^{3} \subset \mathbb{P}(15,10,3,8) \\
\text { Diff }=\left(0,0,0, \frac{2}{3}\right)\end{array}$ & 4 \\
\hline 126 & $x^{10}+a x^{6} y^{5}+x^{2} y^{10}$ & $\begin{array}{c}t^{2}+z+x^{10}+a x^{6} y+x^{2} y^{2} \subset \mathbb{P}(5,10,1,4) \\
\mathbb{P}(5,1,4), \text { Diff }=\left(0, \frac{2}{3}, 0, \frac{4}{5}\right)\end{array}$ & 6 \\
\hline 127 & $x^{10}+x^{2} y^{11}$ & $\begin{array}{c}t^{2}+z+x^{10}+x^{2} y \subset \mathbb{P}(5,10,1,8) \\
\mathbb{P}(5,1,8), \text { Diff }=\left(0, \frac{2}{3}, 0, \frac{10}{11}\right)\end{array}$ & 12 \\
\hline 128 & $\begin{array}{c}x^{10}+x^{3} y^{n} \\
n=8 ; 10\end{array}$ & $\begin{array}{c}t^{2}+z+x^{10}+x^{3} y \subset \mathbb{P}(5,10,1,7) \\
\mathbb{P}(5,1,7), \text { Diff }=\left(0, \frac{2}{3}, 0, \frac{n-1}{n}\right)\end{array}$ & 9,21 \\
\hline 129 & $\begin{array}{l}x^{10}+a z x^{2} y^{6}+ \\
+b x^{3} y^{9}\end{array}$ & $\begin{array}{c}t^{2}+z^{3}+g\left(z, x, y^{1 / 3}\right) \subset \mathbb{P}(15,10,3,7) \\
\text { Diff }=\left(0,0,0, \frac{2}{3}\right)\end{array}$ & 7 \\
\hline 130 & $x^{10}+x^{4} y^{7}$ & $\begin{array}{c}t^{2}+z+x^{10}+x^{4} y \subset \mathbb{P}(5,10,1,6) \\
\mathbb{P}(5,1,6), \text { Diff }=\left(0, \frac{2}{3}, 0, \frac{6}{7}\right)\end{array}$ & 9 \\
\hline 131 & $\begin{array}{c}x^{10}+z y^{n} \\
n=7 ; 9\end{array}$ & $\begin{array}{c}t^{2}+z^{3}+z y+x^{2} \subset \mathbb{P}(3,2,3,4) \\
\text { Diff }=\left(0,0, \frac{4}{5}, \frac{n-1}{n}\right)\end{array}$ & 7,10 \\
\hline 132 & $\begin{array}{c}x^{10}+z x y^{n} \\
n=6,7 ; 8\end{array}$ & $\begin{array}{c}t^{2}+z^{3}+z x y+x^{10} \subset \mathbb{P}(15,10,3,17) \\
\text { Diff }=\left(0,0,0, \frac{n-1}{n}\right)\end{array}$ & 7,17 \\
\hline 133 & $x^{10}+z x^{2} y^{5}$ & $\begin{array}{c}t^{2}+z^{3}+z x^{2} y+x^{10} \subset \mathbb{P}(15,10,3,14) \\
\text { Diff }=\left(0,0,0, \frac{4}{5}\right)\end{array}$ & 7 \\
\hline 134 & $x^{10}+z x^{3} y^{5}$ & $\begin{array}{c}t^{2}+z^{3}+z x^{3} y+x^{10} \subset \mathbb{P}(15,10,3,11) \\
\text { Diff }=\left(0,0,0, \frac{4}{5}\right)\end{array}$ & 11 \\
\hline
\end{tabular}


Продолжение таблицы 1

\begin{tabular}{|c|c|c|c|}
\hline 135 & $x^{9} y+a z x^{3} y^{4}+y^{11}$ & $\begin{array}{c}t+z^{3}+g\left(z, x^{1 / 3}, y\right) \subset \mathbb{P}(33,11,10,3) \\
\mathbb{P}(11,10,3), \text { Diff }=\left(\frac{1}{2}, 0, \frac{2}{3}, 0\right)\end{array}$ & 4 \\
\hline 136 & $x^{9} y+a z y^{8}+b y^{12}$ & $\begin{array}{c}t^{2}+z^{3}+a z y^{8}+x y+b y^{12} \subset \mathbb{P}(6,4,11,1) \\
\text { Diff }=\left(0,0, \frac{8}{9}, 0\right)\end{array}$ & 9 \\
\hline 137 & $\begin{array}{l}\underline{a} z z^{2} x^{3 i+2} f_{1-i}\left(x^{3}, y^{4}\right)+ \\
\quad+\underline{b} y x^{3 j} f_{3-j}\left(x^{3}, y^{4}\right)\end{array}$ & $\begin{array}{c}t+z^{3}+g(z, x, y) \subset \mathbb{P}(39,13,4,3) \\
\mathbb{P}(13,4,3), \text { Diff }=\left(\frac{1}{2}, 0,0,0\right)\end{array}$ & 6 \\
\hline 138 & $x^{9} y+a z x^{3} y^{5}+b y^{14}$ & $\begin{array}{c}t^{2}+z^{3}+g\left(z, x^{1 / 3}, y\right) \subset \mathbb{P}(21,14,13,3) \\
\text { Diff }=\left(0,0, \frac{2}{3}, 0\right)\end{array}$ & 9 \\
\hline 139 & $x^{9} y+a z y^{10}+b y^{15}$ & $\begin{array}{c}t+z^{3}+g\left(z, x^{1 / 9}, y\right) \subset \mathbb{P}(15,5,14,1) \\
\mathbb{P}(5,14,1), \text { Diff }=\left(\frac{1}{2}, 0, \frac{8}{9}, 0\right)\end{array}$ & 18 \\
\hline 140 & $\begin{array}{c}x^{9} y+x y^{n} \\
n=10 ; 12 ; 14\end{array}$ & $\begin{array}{l}t+z+g(z, x, y) \subset \mathbb{P}(9 n-1,9 n-1, n-1,8) \\
\quad \mathbb{P}(9 n-1, n-1,8), \text { Diff }=\left(\frac{1}{2}, \frac{2}{3}, 0,0\right)\end{array}$ & $12,30,48$ \\
\hline 141 & $x^{9} y+a x^{5} y^{6}+x y^{11}$ & $\begin{array}{c}t+z+g(z, x, y) \subset \mathbb{P}(49,49,5,4) \\
\mathbb{P}(49,5,4), \text { Diff }=\left(\frac{1}{2}, \frac{2}{3}, 0,0\right)\end{array}$ & 6 \\
\hline 142 & $\begin{array}{c}y x^{2 i+1} f_{4-i}\left(x^{2}, y^{3}\right) \\
i \leqslant 2\end{array}$ & $\begin{array}{c}t+z+g(z, x, y) \subset \mathbb{P}(29,29,3,2) \\
\mathbb{P}(29,3,2), \text { Diff }=\left(\frac{1}{2}, \frac{2}{3}, 0,0\right)\end{array}$ & 12 \\
\hline 143 & $\begin{array}{c}x^{9} y+x^{2} y^{n} \\
n=9,11\end{array}$ & $\begin{array}{c}t+z+g(z, x, y) \subset \mathbb{P}(9 n-2,9 n-2, n-1,7) \\
\quad \mathbb{P}(9 n-2, n-1,7), \text { Diff }=\left(\frac{1}{2}, \frac{2}{3}, 0,0\right)\end{array}$ & 12 \\
\hline 144 & $\begin{array}{c}x^{9} y+x^{2} y^{2 n} \\
n=5 ; 6\end{array}$ & $\begin{array}{c}t^{2}+z+g(z, x, y) \subset \mathbb{P}(9 n-1,18 n-2,2 n-1,7) \\
\mathbb{P}(9 n-1,2 n-1,7), \text { Diff }=\left(0, \frac{2}{3}, 0,0\right)\end{array}$ & 12,21 \\
\hline 145 & $x^{9} y+a z x^{4} y^{3}+x^{3} y^{8}$ & $\begin{array}{c}t+z^{3}+g(z, x, y) \subset \mathbb{P}(69,23,7,6) \\
\mathbb{P}(23,7,6), \text { Diff }=\left(\frac{1}{2}, 0,0,0\right)\end{array}$ & 4 \\
\hline 146 & $\begin{array}{c}x^{9} y+x^{4} y^{n} \\
n=7 ; 9\end{array}$ & $\begin{array}{c}t+z+g(z, x, y) \subset \mathbb{P}(9 n-4,9 n-4, n-1,5) \\
\mathbb{P}(9 n-4, n-1,5), \text { Diff }=\left(\frac{1}{2}, \frac{2}{3}, 0,0\right)\end{array}$ & 18,30 \\
\hline 147 & $x^{9} y+x^{4} y^{8}$ & $\begin{array}{c}t^{2}+z+g(z, x, y) \subset \mathbb{P}(34,68,7,5) \\
\mathbb{P}(34,7,5), \text { Diff }=\left(0, \frac{2}{3}, 0,0\right)\end{array}$ & 15 \\
\hline 148 & $\begin{array}{c}x^{9} y+z y^{n} \\
n=7,9\end{array}$ & $\begin{array}{c}t+z^{3}+x y+z y^{n} \subset \mathbb{P}(3 n, n, 3 n-2,2) \\
\mathbb{P}(n, 3 n-2,2), \text { Diff }=\left(\frac{1}{2}, 0, \frac{8}{9}, 0\right)\end{array}$ & 10 \\
\hline 149 & $\begin{array}{c}x^{9} y+z x y^{n} \\
n=6,8\end{array}$ & $\begin{array}{c}t+z^{3}+g(z, x, y) \subset \mathbb{P}(27 n-3,9 n-1,3 n-2,15) \\
\mathbb{P}(9 n-1,3 n-2,15), \text { Diff }=\left(\frac{1}{2}, 0,0,0\right)\end{array}$ & 10 \\
\hline 150 & $x^{9} y+z x y^{7}$ & $\begin{array}{c}t^{2}+z^{3}+g(z, x, y) \subset \mathbb{P}(93,62,19,15) \\
\text { Diff }=\varnothing\end{array}$ & 5 \\
\hline 151 & $\begin{array}{c}x^{9} y+z x^{2} y^{n} \\
n=5 ; 7\end{array}$ & $\begin{array}{c}t+z^{3}+g(z, x, y) \subset \mathbb{P}(27 n-6,9 n-2,3 n-2,12) \\
\mathbb{P}(9 n-2,3 n-2,12), \text { Diff }=\left(\frac{1}{2}, 0,0,0\right)\end{array}$ & 14,24 \\
\hline 152 & $\begin{array}{c}x^{8} y^{2}+y^{n} \\
n=11 ; 13 ; 17\end{array}$ & $\begin{array}{c}t^{2}+z+x^{2} y^{2}+y^{n} \subset \mathbb{P}(n, 2 n, n-2,2) \\
\mathbb{P}(n, n-2,2), \text { Diff }=\left(0, \frac{2}{3}, \frac{3}{4}, 0\right)\end{array}$ & $16,18,24$ \\
\hline 153 & $x^{8} y^{2}+a z y^{10}+b y^{15}$ & $\begin{array}{c}t^{2}+z^{3}+g\left(z, x^{1 / 4}, y\right) \subset \mathbb{P}(15,10,13,2) \\
\text { Diff }=\left(0,0, \frac{3}{4}, 0\right)\end{array}$ & 8 \\
\hline
\end{tabular}


Продолжение таблицы 1

\begin{tabular}{|c|c|c|c|}
\hline 154 & $\begin{array}{l}a z y^{3} x^{4 i} f_{1-i}\left(x^{4}, y^{5}\right)+ \\
+y^{2} x^{4 j} f_{2-j}\left(x^{4}, y^{5}\right)\end{array}$ & $\begin{array}{c}t^{2}+z^{3}+g\left(z, x^{1 / 4}, y\right) \subset \mathbb{P}(6,4,5,1) \\
\text { Diff }=\left(0,0, \frac{3}{4}, 0\right)\end{array}$ & 4 \\
\hline 155 & $x^{8} y^{2}+a x^{4} y^{9}+b y^{16}$ & $\begin{array}{c}t^{2}+z+x^{2} y^{2}+a x y^{9}+b y^{16} \subset \mathbb{P}(8,16,7,1) \\
\mathbb{P}(8,7,1), \text { Diff }=\left(0, \frac{2}{3}, \frac{3}{4}, 0\right)\end{array}$ & 12 \\
\hline 156 & $\begin{array}{c}y^{2} x^{2 i} f_{4-i}\left(x^{2}, y^{3}\right) \\
i \leqslant 2\end{array}$ & $\begin{array}{c}t^{2}+z+y^{2} x^{i} f_{4-i}\left(x, y^{3}\right) \subset \mathbb{P}(7,14,3,1) \\
\mathbb{P}(7,3,1), \text { Diff }=\left(0, \frac{2}{3}, \frac{1}{2}, 0\right)\end{array}$ & 6 \\
\hline 157 & $\begin{array}{c}x^{8} y^{2}+a z x^{3} y^{n+1}+ \\
+b x y^{3 n+1}, n=3 b \neq 0 ; 4\end{array}$ & $\begin{array}{c}t^{2}+z^{3}+g(z, x, y) \subset \mathbb{P}(12 n+3,8 n+2,3 n-1,7) \\
\text { Diff }=\varnothing\end{array}$ & 4,7 \\
\hline 158 & $\begin{array}{c}x^{8} y^{2}+x y^{n} \\
n=11,12 ; 14 ; 15\end{array}$ & $\begin{array}{c}t^{2}+z+g(z, x, y) \subset \mathbb{P}(4 n-1,8 n-2, n-2,7) \\
\mathbb{P}(4 n-1, n-2,7), \text { Diff }=\left(0, \frac{2}{3}, 0,0\right)\end{array}$ & $15,18,21$ \\
\hline 159 & $\begin{array}{c}x^{8} y^{2}+x^{2} y^{n} \\
n=9 ; 13\end{array}$ & $\begin{array}{c}t^{2}+z+x^{4} y^{2}+x y^{n} \subset \mathbb{P}(2 n-1,4 n-2, n-2,3) \\
\mathbb{P}(2 n-1, n-2,3), \text { Diff }=\left(0, \frac{2}{3}, \frac{1}{2}, 0\right)\end{array}$ & 12,18 \\
\hline 160 & $\begin{array}{c}x^{3 i+2} y^{2} f_{2-i}\left(x^{3}, y^{n-2}\right) \\
n=6 i=0 ; 7 i \leqslant 1\end{array}$ & $\begin{array}{c}t^{2}+z+g(z, x, y) \subset \mathbb{P}(4 n-5,8 n-10, n-2,3) \\
\mathbb{P}(4 n-5, n-2,3), \text { Diff }=\left(0, \frac{2}{3}, 0,0\right)\end{array}$ & 6,9 \\
\hline 161 & $\begin{array}{c}x^{8} y^{2}+x^{3} y^{n} \\
n=8 ; 10 ; 11\end{array}$ & $\begin{array}{c}t^{2}+z+g(z, x, y) \subset \mathbb{P}(4 n-3,8 n-6, n-2,5) \\
\mathbb{P}(4 n-3, n-2,5), \text { Diff }=\left(0, \frac{2}{3}, 0,0\right)\end{array}$ & $9,12,15$ \\
\hline 162 & $x^{8} y^{2}+a z x^{2} y^{6}+b x^{3} y^{9}$ & $\begin{array}{c}t^{2}+z^{3}+g(z, x, y) \subset \mathbb{P}(33,22,7,5) \\
\text { Diff }=\varnothing\end{array}$ & 5 \\
\hline 163 & $\begin{array}{c}x^{8} y^{2}+z y^{n} \\
n=7 ; 9 ; 11\end{array}$ & $\begin{array}{c}t^{2}+z^{3}+x^{2} y^{2}+z y^{n} \subset \mathbb{P}(3 n, 2 n, 3 n-4,4) \\
\text { Diff }=\left(0,0, \frac{3}{4}, 0\right)\end{array}$ & $10,13,16$ \\
\hline 164 & $\begin{array}{c}x^{8} y^{2}+z x y^{n} \\
n=6 ; 7,8 ; 9\end{array}$ & $\begin{array}{c}t^{2}+z^{3}+g(z, x, y) \subset \mathbb{P}(12 n-3,8 n-2,3 n-4,13) \\
\text { Diff }=\varnothing\end{array}$ & $7,10,13$ \\
\hline 165 & $\begin{array}{c}x^{8} y^{2}+z x^{2} y^{n} \\
n=5 ; 7\end{array}$ & $\begin{array}{c}t^{2}+z^{3}+x^{4} y^{2}+z x y^{n} \subset \mathbb{P}(6 n-3,4 n-2,3 n-4,5) \\
\text { Diff }=\left(0,0, \frac{1}{2}, 0\right)\end{array}$ & 7,10 \\
\hline 166 & $\begin{array}{c}\quad x^{7} y^{3}+y^{n} \\
n=11 ; 13 ; 19 ; 23\end{array}$ & $\begin{array}{c}t+z+x y^{3}+y^{n} \subset \mathbb{P}(n, n, n-3,1) \\
\mathbb{P}(n, n-3,1), \text { Diff }=\left(\frac{1}{2}, \frac{2}{3}, \frac{6}{7}, 0\right)\end{array}$ & $\begin{array}{c}18,22,36 \\
42\end{array}$ \\
\hline 167 & $\begin{array}{c}x^{7} y^{3}+y^{2 n} \\
n=7,8 ; 10,11\end{array}$ & $\begin{array}{c}t^{2}+z+x y^{3}+y^{2 n} \subset \mathbb{P}(n, 2 n, 2 n-3,1) \\
\mathbb{P}(n, 2 n-3,1), \text { Diff }=\left(0, \frac{2}{3}, \frac{6}{7}, 0\right)\end{array}$ & 15,21 \\
\hline 168 & $\begin{array}{c}x^{7} y^{3}+a z y^{2 n}+b y^{3 n} \\
n=5 ; 7\end{array}$ & $\begin{array}{l}t+z^{3}+g\left(z, x^{1 / 7}, y\right) \subset \mathbb{P}(3 n, n, 3 n-3,1) \\
\mathbb{P}(n, 3 n-3,1), \text { Diff }=\left(\frac{1}{2}, 0, \frac{6}{7}, 0\right)\end{array}$ & 8,14 \\
\hline 169 & $x^{7} y^{3}+a z y^{8}+b y^{12}$ & $\begin{array}{c}t^{2}+z^{3}+g\left(z, x^{1 / 7}, y\right) \subset \mathbb{P}(6,4,9,1) \\
\text { Diff }=\left(0,0, \frac{6}{7}, 0\right)\end{array}$ & 7 \\
\hline 170 & $x^{7} y^{3}+a z y^{12}+b y^{18}$ & $\begin{array}{c}t^{2}+z^{3}+g\left(z, x^{1 / 7}, y^{1 / 3}\right) \subset \mathbb{P}(3,2,5,1) \\
\text { Diff }=\left(0,0, \frac{6}{7}, \frac{2}{3}\right)\end{array}$ & 7 \\
\hline 171 & $\begin{array}{c}y^{3} x^{i} f_{7-i}\left(x, y^{2}\right) \\
i \leqslant 5\end{array}$ & $\begin{array}{c}t+z+g(z, x, y) \subset \mathbb{P}(17,17,2,1) \\
\mathbb{P}(17,2,1), \text { Diff }=\left(\frac{1}{2}, \frac{2}{3}, 0,0\right)\end{array}$ & 6 \\
\hline 172 & $\begin{array}{c}x^{7} y^{3}+x y^{n} \\
n=10 ; 14 ; 16 ; 20\end{array}$ & $\begin{array}{c}t+z+g(z, x, y) \subset \mathbb{P}(7 n-3,7 n-3, n-3,6) \\
\mathbb{P}(7 n-3, n-3,6), \text { Diff }=\left(\frac{1}{2}, \frac{2}{3}, 0,0\right)\end{array}$ & $\begin{array}{c}16,24,30 \\
36\end{array}$ \\
\hline
\end{tabular}


Продолжение таблицы 1

\begin{tabular}{|c|c|c|c|}
\hline 173 & $\begin{array}{c}x^{7} y^{3}+a x^{4} y^{n+2}+b x y^{2 n+1} \\
n=5 ; 9\end{array}$ & $\begin{array}{l}t+z+g(z, x, y) \subset \mathbb{P}(7 n+2,7 n+2, n-1,3) \\
\quad \mathbb{P}(7 n+2, n-1,3), \text { Diff }=\left(\frac{1}{2}, \frac{2}{3}, 0,0\right)\end{array}$ & 12,18 \\
\hline 174 & $\begin{array}{c}x^{7} y^{3}+a x^{4} y^{2 n+2}+b x y^{4 n+1} \\
n=3,4\end{array}$ & $\begin{array}{c}t^{2}+z+g(z, x, y) \subset \mathbb{P}(7 n+1,14 n+2,2 n-1,3) \\
\mathbb{P}(7 n+1,2 n-1,3), \text { Diff }=\left(0, \frac{2}{3}, 0,0\right)\end{array}$ & 9 \\
\hline 175 & $\begin{array}{c}y^{3} x^{2 i+1} f_{3-i}\left(x^{2}, y^{3}\right) \\
i=0,1\end{array}$ & $\begin{array}{c}t+z^{3}+y x^{2 i+1} f_{3-i}\left(x^{2}, y\right) \subset \mathbb{P}(9,3,1,2) \\
\mathbb{P}(3,1,2), \text { Diff }=\left(\frac{1}{2}, 0,0, \frac{2}{3}\right)\end{array}$ & 4 \\
\hline 176 & $\begin{array}{c}y^{3} x^{2 i+1} f_{3-i}\left(x^{2}, y^{5}\right) \\
i \leqslant 2\end{array}$ & $\begin{array}{c}t+z+g(z, x, y) \subset \mathbb{P}(41,41,5,2) \\
\mathbb{P}(41,5,2), \text { Diff }=\left(\frac{1}{2}, \frac{2}{3}, 0,0\right)\end{array}$ & 12 \\
\hline 177 & $x^{7} y^{3}+a z x^{3} y^{4}+x^{2} y^{9}$ & $\begin{array}{c}t+z^{3}+g(z, x, y) \subset \mathbb{P}(57,19,6,5) \\
\mathbb{P}(19,6,5), \text { Diff }=\left(\frac{1}{2}, 0,0,0\right)\end{array}$ & 4 \\
\hline 178 & $\begin{array}{c}x^{7} y^{3}+x^{2} y^{2 n} \\
n=5 ; 7,8\end{array}$ & $\begin{array}{c}t^{2}+z+g(z, x, y) \subset \mathbb{P}(7 n-3,14 n-6,2 n-3,5) \\
\mathbb{P}(7 n-3,2 n-3,5), \text { Diff }=\left(0, \frac{2}{3}, 0,0\right)\end{array}$ & 9,15 \\
\hline 179 & $\begin{array}{c}x^{7} y^{3}+x^{2} y^{n} \\
n=11 ; 17\end{array}$ & $\begin{array}{c}t+z+g(z, x, y) \subset \mathbb{P}(7 n-6,7 n-6, n-3,5) \\
\mathbb{P}(7 n-6, n-3,5), \text { Diff }=\left(\frac{1}{2}, \frac{2}{3}, 0,0\right)\end{array}$ & 18,30 \\
\hline 180 & $x^{7} y^{3}+a z x^{3} y^{5}+b x^{2} y^{12}$ & $\begin{array}{c}t^{2}+z^{3}+g(z, x, y) \subset \mathbb{P}(39,26,9,5) \\
\text { Diff }=\varnothing\end{array}$ & 5 \\
\hline 181 & $x^{7} y^{3}+a z x^{3} y^{6}+b x^{2} y^{15}$ & $\begin{array}{l}t+z^{3}+g\left(z, x, y^{1 / 3}\right) \subset \mathbb{P}(33,11,4,5) \\
\quad \mathbb{P}(11,4,5), \text { Diff }=\left(\frac{1}{2}, 0,0, \frac{2}{3}\right)\end{array}$ & 10 \\
\hline 182 & $\begin{array}{l}x^{7} y^{3}+x^{3} y^{n} \\
n=8 ; 10 ; 14\end{array}$ & $\begin{array}{c}t+z+g(z, x, y) \subset \mathbb{P}(7 n-9,7 n-9, n-3,4) \\
\mathbb{P}(7 n-9, n-3,4), \text { Diff }=\left(\frac{1}{2}, \frac{2}{3}, 0,0\right)\end{array}$ & $12,18,24$ \\
\hline 183 & $x^{7} y^{3}+a z x^{2} y^{8}+b x^{3} y^{12}$ & $\begin{array}{c}t+z^{3}+g(z, x, y) \subset \mathbb{P}(75,25,9,4) \\
\mathbb{P}(25,9,4), \text { Diff }=\left(\frac{1}{2}, 0,0,0\right)\end{array}$ & 8 \\
\hline 184 & $\begin{array}{l}x^{7} y^{3}+z y^{n} \\
n=7 ; 11,13\end{array}$ & $\begin{array}{c}t+z^{3}+x y^{3}+z y^{n} \subset \mathbb{P}(3 n, n, 3 n-6,2) \\
\mathbb{P}(n, 3 n-6,2), \text { Diff }=\left(\frac{1}{2}, 0, \frac{6}{7}, 0\right)\end{array}$ & 10,22 \\
\hline 185 & $x^{7} y^{3}+z y^{15}$ & $\begin{array}{c}t+z^{3}+x y+z y^{5} \subset \mathbb{P}(15,5,13,2) \\
\mathbb{P}(5,13,2), \text { Diff }=\left(\frac{1}{2}, 0, \frac{6}{7}, \frac{2}{3}\right)\end{array}$ & 28 \\
\hline 186 & $\begin{array}{c}x^{7} y^{3}+z x y^{3 n} \\
n=2 ; 4\end{array}$ & $\begin{array}{c}t+z^{3}+x^{7} y+z x y^{n} \subset \mathbb{P}(21 n-3,7 n-1,3 n-2,11) \\
\mathbb{P}(7 n-1,3 n-6,11), \text { Diff }=\left(\frac{1}{2}, 0,0, \frac{2}{3}\right)\end{array}$ & 10,22 \\
\hline 187 & $\begin{array}{c}x^{7} y^{3}+z x y^{n} \\
n=7 ; 11\end{array}$ & $\begin{array}{c}t^{2}+z^{3}+g(z, x, y) \subset \mathbb{P}(21 n-9,14 n-6,6 n-12,22) \\
\text { Diff }=\varnothing\end{array}$ & 5,11 \\
\hline 188 & $\begin{array}{c}x^{7} y^{3}+z x y^{2 n} \\
n=4,5\end{array}$ & $\begin{array}{c}t+z^{3}+g(z, x, y) \subset \mathbb{P}(42 n-9,14 n-3,6 n-6,11) \\
\mathbb{P}(14 n-3,6 n-6,11), \text { Diff }=\left(\frac{1}{2}, 0,0,0\right)\end{array}$ & 16 \\
\hline 189 & $x^{7} y^{3}+z x y^{9}$ & $\begin{array}{c}t^{2}+z^{3}+x^{7} y+z x y^{3} \subset \mathbb{P}(30,20,7,11) \\
\text { Diff }=\left(0,0,0, \frac{2}{3}\right)\end{array}$ & 11 \\
\hline 190 & $\begin{array}{c}x^{7} y^{3}+z x^{2} y^{n} \\
n=5,7\end{array}$ & $\begin{array}{c}t+z^{3}+g(z, x, y) \subset \mathbb{P}(21 n-18,7 n-6,3 n-6,8) \\
\mathbb{P}(7 n-6,3 n-6,8), \text { Diff }=\left(\frac{1}{2}, 0,0,0\right)\end{array}$ & 10 \\
\hline 191 & $x^{7} y^{3}+z x^{2} y^{9}$ & $\begin{array}{c}t+z^{3}+x^{7} y+z x^{2} y^{3} \subset \mathbb{P}(57,19,7,8) \\
\mathbb{P}(19,7,8), \text { Diff }=\left(\frac{1}{2}, 0,0, \frac{2}{3}\right)\end{array}$ & 16 \\
\hline
\end{tabular}


Продолжение таблицы 1

\begin{tabular}{|c|c|c|c|}
\hline 192 & $\begin{array}{c}x^{i} y^{j} f_{11-i-j}(x, y) \\
i \leqslant 4 \& j \leqslant 4\end{array}$ & $\begin{array}{l}t+z+g(z, x, y) \subset \mathbb{P}(11,11,1,1) \\
\mathbb{P}(11,1,1), \text { Diff }=\left(\frac{1}{2}, \frac{2}{3}, 0,0\right)\end{array}$ & 6 \\
\hline 193 & $x^{11}+a z y^{8}+b y^{12}$ & $\begin{array}{c}t^{2}+z^{3}+x+a z y^{4}+b y^{6} \subset \mathbb{P}(3,2,6,1) \\
\mathbb{P}(3,2,1), \text { Diff }=\left(0,0, \frac{10}{11}, \frac{1}{2}\right)\end{array}$ & 11 \\
\hline 194 & $x^{11}+y^{13}$ & $\begin{array}{l}t+z+x+y \subset \mathbb{P}(1,1,1,1) \\
\mathbb{P}^{2}, \text { Diff }=\left(\frac{1}{2}, \frac{2}{3}, \frac{10}{11}, \frac{12}{13}\right)\end{array}$ & 66 \\
\hline 195 & $x^{11}+x y^{11}$ & $\begin{array}{l}t+z+x^{11}+x y \subset \mathbb{P}(11,11,1,10) \\
\mathbb{P}(11,1,10), \text { Diff }=\left(\frac{1}{2}, \frac{2}{3}, 0, \frac{10}{11}\right)\end{array}$ & 12 \\
\hline 196 & $x^{11}+x^{2} y^{10}$ & $\begin{array}{c}t^{2}+z+x^{11}+x^{2} y^{2} \subset \mathbb{P}(11,22,2,9) \\
\mathbb{P}(11,2,9), \text { Diff }=\left(0, \frac{2}{3}, 0, \frac{4}{5}\right)\end{array}$ & 15 \\
\hline 197 & $x^{11}+a z x^{2} y^{6}+b x^{3} y^{9}$ & $\begin{array}{c}t+z^{3}+g\left(z, x, y^{1 / 2}\right) \subset \mathbb{P}(33,11,3,8) \\
\mathbb{P}(11,3,8), \text { Diff }=\left(\frac{1}{2}, 0,0, \frac{2}{3}\right)\end{array}$ & 16 \\
\hline 198 & $x^{11}+x^{4} y^{8}$ & $\begin{array}{c}t^{2}+z+x^{11}+x^{4} y^{2} \subset \mathbb{P}(11,22,2,7) \\
\mathbb{P}(11,2,7), \text { Diff }=\left(0, \frac{2}{3}, 0, \frac{3}{4}\right)\end{array}$ & 21 \\
\hline 199 & $x^{11}+x^{5} y^{7}$ & $\begin{array}{c}t+z+x^{11}+x^{5} y \subset \mathbb{P}(11,11,1,6) \\
\mathbb{P}(11,1,6), \text { Diff }=\left(\frac{1}{2}, \frac{2}{3}, 0, \frac{6}{7}\right)\end{array}$ & 36 \\
\hline 200 & $x^{11}+z x y^{7}$ & $\begin{array}{c}t+z^{3}+x^{11}+z x y \subset \mathbb{P}(33,11,3,19) \\
\mathbb{P}(11,3,19), \text { Diff }=\left(\frac{1}{2}, 0,0, \frac{6}{7}\right)\end{array}$ & 14 \\
\hline 201 & $x^{11}+z x^{3} y^{5}$ & $\begin{array}{l}t+z^{3}+x^{11}+z x^{3} y \subset \mathbb{P}(33,11,3,13) \\
\mathbb{P}(11,3,13), \text { Diff }=\left(\frac{1}{2}, 0,0, \frac{4}{5}\right)\end{array}$ & 26 \\
\hline 202 & $x^{10} y+a z y^{8}+b y^{12}$ & $\begin{array}{c}t^{2}+z^{3}+g\left(z, x^{1 / 10}, y\right) \subset \mathbb{P}(6,4,11,1) \\
\text { Diff }=\left(0,0, \frac{9}{10}, 0\right)\end{array}$ & 10 \\
\hline 203 & $x^{10} y+a x^{5} y^{7}+b y^{13}$ & $\begin{array}{c}t+z+x^{2} y+a x y^{7}+b y^{13} \subset \mathbb{P}(13,13,6,1) \\
\mathbb{P}(13,6,1), \text { Diff }=\left(\frac{1}{2}, \frac{2}{3}, \frac{4}{5}, 0\right)\end{array}$ & 30 \\
\hline 204 & $\begin{array}{c}x^{10} y+x y^{n} \\
n=11 ; 12\end{array}$ & $\begin{array}{c}t+z+g(z, x, y) \subset \mathbb{P}(10 n-1,10 n-1, n-1,9) \\
\mathbb{P}(10 n-1, n-1,9), \text { Diff }=\left(\frac{1}{2}, \frac{2}{3}, 0,0\right)\end{array}$ & 12,54 \\
\hline 205 & $x^{10} y+x^{2} y^{10}$ & $\begin{array}{c}t^{2}+z+g(z, x, y) \subset \mathbb{P}(49,98,9,8) \\
\mathbb{P}(49,9,8), \text { Diff }=\left(0, \frac{2}{3}, 0,0\right)\end{array}$ & 12 \\
\hline 206 & $x^{10} y+a z x^{2} y^{6}+b x^{3} y^{9}$ & $\begin{array}{c}t+z^{3}+g(z, x, y) \subset \mathbb{P}(87,29,8,7) \\
\mathbb{P}(29,8,7), \text { Diff }=\left(\frac{1}{2}, 0,0,0\right)\end{array}$ & 14 \\
\hline 207 & $x^{10} y+x^{4} y^{8}$ & $\begin{array}{c}t^{2}+z+x^{5} y+x^{2} y^{8} \subset \mathbb{P}(19,38,7,3) \\
\mathbb{P}(19,7,3), \text { Diff }=\left(0, \frac{2}{3}, \frac{1}{2}, 0\right)\end{array}$ & 18 \\
\hline 208 & $x^{10} y+z x y^{7}$ & $\begin{array}{l}t+z^{3}+g(z, x, y) \subset \mathbb{P}(207,69,19,17) \\
\quad \mathbb{P}(69,19,17), \text { Diff }=\left(\frac{1}{2}, 0,0,0\right)\end{array}$ & 24 \\
\hline 209 & $x^{10} y+z x^{3} y^{5}$ & $\begin{array}{c}t+z^{3}+g(z, x, y) \subset \mathbb{P}(141,47,13,11) \\
\mathbb{P}(47,13,11), \operatorname{Diff}=\left(\frac{1}{2}, 0,0,0\right)\end{array}$ & 22 \\
\hline 210 & $x^{9} y^{2}+a z y^{8}+b y^{12}$ & $\begin{array}{c}t^{2}+z^{3}+x y+a z y^{4}+b y^{6} \subset \mathbb{P}(3,2,5,1) \\
\text { Diff }=\left(0,0, \frac{8}{9}, \frac{1}{2}\right)\end{array}$ & 9 \\
\hline
\end{tabular}


Продолжение таблицы 1

\begin{tabular}{|c|c|c|c|}
\hline 211 & $x^{9} y^{2}+a z x^{3} y^{5}+b y^{13}$ & $\begin{array}{c}t+z^{3}+g\left(z, x^{1 / 3}, y\right) \subset \mathbb{P}(39,13,11,3) \\
\mathbb{P}(13,11,3), \text { Diff }=\left(\frac{1}{2}, 0, \frac{2}{3}, 0\right)\end{array}$ & 18 \\
\hline 212 & $x^{9} y^{2}+x y^{11}$ & $\begin{array}{c}t+z+g(z, x, y) \subset \mathbb{P}(97,97,9,8) \\
\mathbb{P}(97,9,8), \text { Diff }=\left(\frac{1}{2}, \frac{2}{3}, 0,0\right)\end{array}$ & 30 \\
\hline 213 & $x^{9} y^{2}+a x^{5} y^{7}+b x y^{12}$ & $\begin{array}{c}t+z+g(z, x, y) \subset \mathbb{P}(53,53,5,4) \\
\mathbb{P}(53,5,4), \text { Diff }=\left(\frac{1}{2}, \frac{2}{3}, 0,0\right)\end{array}$ & 24 \\
\hline 214 & $x^{9} y^{2}+x^{2} y^{10}$ & $\begin{array}{c}t^{2}+z+g(z, x, y) \subset \mathbb{P}(43,86,8,7) \\
\mathbb{P}(43,8,7), \text { Diff }=\left(0, \frac{2}{3}, 0,0\right)\end{array}$ & 12 \\
\hline 215 & $x^{9} y^{2}+x^{2} y^{11}$ & $\begin{array}{c}t+z+g(z, x, y) \subset \mathbb{P}(95,95,9,7) \\
\mathbb{P}(95,9,7), \text { Diff }=\left(\frac{1}{2}, \frac{2}{3}, 0,0\right)\end{array}$ & 42 \\
\hline 216 & $x^{9} y^{2}+a z x^{2} y^{6}+b x^{3} y^{9}$ & $\begin{array}{c}t+z^{3}+g(z, x, y) \subset \mathbb{P}(75,25,7,6) \\
\mathbb{P}(25,7,6), \text { Diff }=\left(\frac{1}{2}, 0,0,0\right)\end{array}$ & 12 \\
\hline 217 & $x^{9} y^{2}+x^{4} y^{8}$ & $\begin{array}{c}t^{2}+z+x^{9} y+x^{4} y^{4} \subset \mathbb{P}(16,32,3,5) \\
\mathbb{P}(16,3,5), \text { Diff }=\left(0, \frac{2}{3}, 0, \frac{1}{2}\right)\end{array}$ & 15 \\
\hline 218 & $x^{9} y^{2}+z y^{9}$ & $\begin{array}{l}t+z^{3}+x y^{2}+z y^{9} \subset \mathbb{P}(27,9,23,2) \\
\mathbb{P}(9,23,2), \text { Diff }=\left(\frac{1}{2}, 0, \frac{8}{9}, 0\right)\end{array}$ & 36 \\
\hline 219 & $x^{9} y^{2}+z x y^{7}$ & $\begin{array}{l}t+z^{3}+g(z, x, y) \subset \mathbb{P}(183,61,17,15) \\
\quad \mathbb{P}(61,17,15), \text { Diff }=\left(\frac{1}{2}, 0,0,0\right)\end{array}$ & 10 \\
\hline 220 & $x^{8} y^{3}+a z y^{8}+b y^{12}$ & $\begin{array}{c}t^{2}+z^{3}+g\left(z, x^{1 / 8}, y\right) \subset \mathbb{P}(6,4,9,1) \\
\text { Diff }=\left(0,0, \frac{7}{8}, 0\right)\end{array}$ & 8 \\
\hline 221 & $x^{8} y^{3}+a x^{4} y^{8}+b y^{13}$ & $\begin{array}{c}t^{2}+z+x^{4} y^{3}+a x^{2} y^{8}+b y^{13} \subset \mathbb{P}(13,26,5,2) \\
\mathbb{P}(13,5,2), \text { Diff }=\left(0, \frac{2}{3}, \frac{1}{2}, 0\right)\end{array}$ & 12 \\
\hline 222 & $x^{8} y^{3}+y^{14}$ & $\begin{array}{c}t^{2}+z+x y^{3}+y^{14} \subset \mathbb{P}(7,14,11,1) \\
\mathbb{P}(7,11,1), \text { Diff }=\left(0, \frac{2}{3}, \frac{7}{8}, 0\right)\end{array}$ & 24 \\
\hline 223 & $\begin{array}{c}x^{8} y^{3}+x y^{n} \\
n=11 ; 13\end{array}$ & $\begin{array}{c}t+z+g(z, x, y) \subset \mathbb{P}(8 n-3,8 n-3, n-3,7) \\
\mathbb{P}(8 n-3, n-3,7), \text { Diff }=\left(\frac{1}{2}, \frac{2}{3}, 0,0\right)\end{array}$ & 18,42 \\
\hline 224 & $x^{8} y^{3}+a z x^{3} y^{5}+b x y^{12}$ & $\begin{array}{c}t+z^{3}+g(z, x, y) \subset \mathbb{P}(93,31,9,7) \\
\mathbb{P}(31,9,7), \text { Diff }=\left(\frac{1}{2}, 0,0,0\right)\end{array}$ & 14 \\
\hline 225 & $x^{8} y^{3}+x^{2} y^{10}$ & $\begin{array}{c}t^{2}+z+g(z, x, y) \subset \mathbb{P}(37,74,7,6) \\
\mathbb{P}(37,7,6), \text { Diff }=\left(0, \frac{2}{3}, 0,0\right)\end{array}$ & 9 \\
\hline 226 & $x^{8} y^{3}+a x^{5} y^{7}+b x^{2} y^{11}$ & $\begin{array}{c}t+z+g(z, x, y) \subset \mathbb{P}(41,41,4,3) \\
\mathbb{P}(41,4,3), \text { Diff }=\left(\frac{1}{2}, \frac{2}{3}, 0,0\right)\end{array}$ & 18 \\
\hline 227 & $\begin{array}{l}\underline{d} x^{8} y^{3}+z\left(\underline{a} x^{7}+b x^{2} y^{6}\right)+ \\
\quad+c x^{3} y^{9},|b|+|c| \neq 0\end{array}$ & $\begin{array}{c}t+z^{3}+g\left(z, x, y^{1 / 3}\right) \subset \mathbb{P}(21,7,2,5) \\
\mathbb{P}(7,2,5), \text { Diff }=\left(\frac{1}{2}, 0,0, \frac{2}{3}\right)\end{array}$ & 10 \\
\hline 228 & $x^{8} y^{3}+x^{3} y^{10}$ & $\begin{array}{c}t+z+g(z, x, y) \subset \mathbb{P}(71,71,7,5) \\
\mathbb{P}(71,7,5), \text { Diff }=\left(\frac{1}{2}, \frac{2}{3}, 0,0\right)\end{array}$ & 30 \\
\hline 229 & $x^{8} y^{3}+z y^{9}$ & $\begin{array}{c}t^{2}+z^{3}+x^{2} y+z y^{3} \subset \mathbb{P}(9,6,7,4) \\
\text { Diff }=\left(0,0, \frac{3}{4}, \frac{2}{3}\right)\end{array}$ & 16 \\
\hline
\end{tabular}


Продолжение таблицы 1

\begin{tabular}{|c|c|c|c|}
\hline 230 & $\begin{array}{c}x^{8} y^{3}+z x y^{n} \\
n=7 ; 8\end{array}$ & $\begin{array}{c}t+z^{3}+g(z, x, y) \subset \mathbb{P}(24 n-9,8 n-3,3 n-6,13) \\
\mathbb{P}(8 n-3,3 n-6,13), \text { Diff }=\left(\frac{1}{2}, 0,0,0\right)\end{array}$ & 10,26 \\
\hline 231 & $\begin{array}{l}\underline{d} x^{7} y^{4}+z\left(\underline{a} x^{7}+b y^{8}\right)+ \\
\quad+c y^{12},|b|+|c| \neq 0\end{array}$ & $\begin{array}{c}t^{2}+z^{3}+g\left(z, x^{1 / 7}, y^{1 / 2}\right) \subset \mathbb{P}(3,2,4,1) \\
\text { Diff }=\left(0,0, \frac{6}{7}, \frac{1}{2}\right)\end{array}$ & 7 \\
\hline 232 & $\begin{array}{c}d x^{7} y^{4}+y^{n} \\
n=13 ; 17\end{array}$ & $\begin{array}{c}t+z+x y^{4}+y^{n} \subset \mathbb{P}(n, n, n-4,1) \\
\mathbb{P}(n, n-4,1), \text { Diff }=\left(\frac{1}{2}, \frac{2}{3}, \frac{6}{7}, 0\right)\end{array}$ & 30,42 \\
\hline 233 & $x^{7} y^{4}+y^{14}$ & $\begin{array}{c}t^{2}+z+x y^{4}+y^{14} \subset \mathbb{P}(7,14,10,1) \\
\mathbb{P}(7,10,1), \text { Diff }=\left(0, \frac{2}{3}, \frac{6}{7}, 0\right)\end{array}$ & 15 \\
\hline 234 & $x^{7} y^{4}+a z y^{10}+b y^{15}$ & $\begin{array}{l}t+z^{3}+g\left(z, x^{1 / 7}, y\right) \subset \mathbb{P}(15,5,11,1) \\
\quad \mathbb{P}(5,11,1), \text { Diff }=\left(\frac{1}{2}, 0, \frac{6}{7}, 0\right)\end{array}$ & 14 \\
\hline 235 & $x^{7} y^{4}+y^{16}$ & $\begin{array}{l}t^{2}+z+x y+y^{4} \subset \mathbb{P}(2,4,3,1) \\
\mathbb{P}(2,3,1), \text { Diff }=\left(0, \frac{2}{3}, \frac{6}{7}, \frac{3}{4}\right)\end{array}$ & 21 \\
\hline 236 & $\begin{array}{c}x^{7} y^{4}+x y^{n} \\
n=11 ; 15\end{array}$ & $\begin{array}{c}t+z+g(z, x, y) \subset \mathbb{P}(7 n-4,7 n-4, n-4,6) \\
\mathbb{P}(7 n-4, n-4,6), \text { Diff }=\left(\frac{1}{2}, \frac{2}{3}, 0,0\right)\end{array}$ & 24,36 \\
\hline 237 & $x^{7} y^{4}+a x^{4} y^{8}+b x y^{12}$ & $\begin{array}{c}t^{2}+z+x^{7} y+a x^{4} y^{2}+b x y^{3} \subset \mathbb{P}(5,10,1,3) \\
\mathbb{P}(5,1,3), \text { Diff }=\left(0, \frac{2}{3}, 0, \frac{3}{4}\right)\end{array}$ & 9 \\
\hline 238 & $x^{7} y^{4}+a x^{4} y^{9}+b x y^{14}$ & $\begin{array}{c}t+z+g(z, x, y) \subset \mathbb{P}(47,47,5,3) \\
\mathbb{P}(47,5,3), \text { Diff }=\left(\frac{1}{2}, \frac{2}{3}, 0,0\right)\end{array}$ & 18 \\
\hline 239 & $\begin{array}{c}x^{2 i+1} y^{4} f_{3-i}\left(x^{2}, y^{3}\right) \\
i \leqslant 2\end{array}$ & $\begin{array}{c}t+z+g(z, x, y) \subset \mathbb{P}(29,29,3,2) \\
\mathbb{P}(29,3,2), \text { Diff }=\left(\frac{1}{2}, \frac{2}{3}, 0,0\right)\end{array}$ & 12 \\
\hline 240 & $x^{7} y^{4}+x^{2} y^{10}$ & $\begin{array}{c}t^{2}+z+g(z, x, y) \subset \mathbb{P}(31,62,6,5) \\
\mathbb{P}(31,6,5), \text { Diff }=\left(0, \frac{2}{3}, 0,0\right)\end{array}$ & 9 \\
\hline 241 & $x^{7} y^{4}+a z x^{3} y^{5}+b x^{2} y^{11}$ & $\begin{array}{c}t+z^{3}+g(z, x, y) \subset \mathbb{P}(69,23,7,5) \\
\mathbb{P}(23,7,5), \text { Diff }=\left(\frac{1}{2}, 0,0,0\right)\end{array}$ & 10 \\
\hline 242 & $x^{7} y^{4}+x^{2} y^{12}$ & $\begin{array}{c}t^{2}+z+x^{7} y^{2}+x^{2} y^{6} \subset \mathbb{P}(19,38,4,5) \\
\mathbb{P}(19,4,5), \text { Diff }=\left(0, \frac{2}{3}, 0, \frac{1}{2}\right)\end{array}$ & 15 \\
\hline 243 & $x^{7} y^{4}+x^{2} y^{13}$ & $\begin{array}{c}t+z+g(z, x, y) \subset \mathbb{P}(83,83,9,5) \\
\mathbb{P}(83,9,5), \text { Diff }=\left(\frac{1}{2}, \frac{2}{3}, 0,0\right)\end{array}$ & 30 \\
\hline 244 & $\begin{array}{c}\underline{d} x^{7} y^{4}+z\left(\underline{a} x^{6} y+b x^{2} y^{6}\right)+ \\
+c x^{3} y^{9},|b|+|c| \neq 0\end{array}$ & $\begin{array}{c}t+z^{3}+g(z, x, y) \subset \mathbb{P}(51,17,5,4) \\
\mathbb{P}(17,5,4), \text { Diff }=\left(\frac{1}{2}, 0,0,0\right)\end{array}$ & 8 \\
\hline 245 & $x^{7} y^{4}+x^{3} y^{11}$ & $\begin{array}{c}t+z+g(z, x, y) \subset \mathbb{P}(65,65,7,4) \\
\mathbb{P}(65,7,4), \text { Diff }=\left(\frac{1}{2}, \frac{2}{3}, 0,0\right)\end{array}$ & 24 \\
\hline 246 & $\begin{array}{c}x^{7} y^{4}+z y^{n} \\
n=9 ; 11\end{array}$ & $\begin{array}{l}t+z^{3}+x y^{4}+z y^{n} \subset \mathbb{P}(3 n, n, 3 n-8,2) \\
\quad \mathbb{P}(n, 3 n-8,2), \text { Diff }=\left(\frac{1}{2}, 0, \frac{6}{7}, 0\right)\end{array}$ & 22,28 \\
\hline 247 & $\begin{array}{c}x^{7} y^{4}+z x y^{n} \\
n=7 ; 9\end{array}$ & $\begin{array}{c}t+z^{3}+g(z, x, y) \subset \mathbb{P}(21 n-12,7 n-4,3 n-8,11) \\
\mathbb{P}(7 n-4,3 n-8,11), \text { Diff }=\left(\frac{1}{2}, 0,0,0\right)\end{array}$ & 16,22 \\
\hline 248 & $x^{7} y^{4}+z x y^{8}$ & $\begin{array}{c}t^{2}+z^{3}+x^{7} y^{2}+z x y^{4} \subset \mathbb{P}(39,26,8,11) \\
\text { Diff }=\left(0,0,0, \frac{1}{2}\right)\end{array}$ & 11 \\
\hline
\end{tabular}


Продолжение таблицы 1

\begin{tabular}{|c|c|c|c|}
\hline 249 & $x^{7} y^{4}+z x^{2} y^{7}$ & $\begin{array}{l}t+z^{3}+g(z, x, y) \subset \mathbb{P}(123,41,13,8) \\
\quad \mathbb{P}(41,13,8), \text { Diff }=\left(\frac{1}{2}, 0,0,0\right)\end{array}$ & 16 \\
\hline 250 & $\begin{aligned} & z x^{5}+y^{2 n} \\
n= & 4 ; 7 ; 8,11 ; 13,14\end{aligned}$ & $\begin{array}{c}t^{2}+z^{3}+z x+y^{2} \subset \mathbb{P}(3,2,4,3) \\
\text { Diff }=\left(0,0, \frac{4}{5}, \frac{n-1}{n}\right)\end{array}$ & $\begin{array}{c}5,7,11 \\
15\end{array}$ \\
\hline 251 & $\begin{array}{c}z x^{5}+y^{n} \\
n=11 ; 13 ; 17 ; 19 ; 23 ; 29\end{array}$ & $\begin{array}{c}t+z^{3}+z x+y \subset \mathbb{P}(3,1,2,3) \\
\mathbb{P}(1,2,3), \text { Diff }=\left(\frac{1}{2}, 0, \frac{4}{5}, \frac{n-1}{n}\right)\end{array}$ & $\begin{array}{l}11,14,18, \\
20,26,30\end{array}$ \\
\hline 252 & $\begin{array}{c}z\left(x^{5}+a y^{2 n}\right)+b y^{3 n}+ \\
+c x^{5} y^{n}, n=3 \\
|a|+|b| \neq 0 ; 7,9\end{array}$ & $\begin{array}{c}t+z^{3}+z\left(x+a y^{2}\right)+b y^{3}+c x y \subset \mathbb{P}(3,1,2,1) \\
\mathbb{P}(1,2,1), \text { Diff }=\left(\frac{1}{2}, 0, \frac{4}{5}, \frac{n-1}{n}\right)\end{array}$ & 6,10 \\
\hline 253 & $\begin{array}{c}z\left(x^{5}+a y^{4 n}\right)+b y^{6 n}+ \\
\quad+c x^{5} y^{2 n} \\
(n=2,3) \&|a|+|b| \neq 0 ; 4\end{array}$ & $\begin{array}{c}t^{2}+z^{3}+z\left(x+a y^{4}\right)+b y^{6}+c x y^{2} \subset \mathbb{P}(3,2,4,1) \\
\text { Diff }=\left(0,0, \frac{4}{5}, \frac{n-1}{n}\right)\end{array}$ & 5 \\
\hline 254 & $\begin{array}{c}z x^{2+3 i} f_{1-i}\left(x^{3}, y^{4 n}\right)+ \\
+a y^{2 n} x^{3 j} f_{2-j}\left(x^{3}, y^{4 n}\right) \\
n=1 j=0 ; 2\end{array}$ & $\begin{array}{c}t^{2}+z^{3}+g\left(z, x, y^{1 / n}\right) \subset \mathbb{P}(15,10,4,3) \\
\text { Diff }=\left(0,0,0, \frac{n-1}{n}\right)\end{array}$ & 2,3 \\
\hline 255 & $\begin{array}{c}z\left(x^{5}+a x^{2} y^{10}\right)+b x^{3} y^{15}+ \\
+c x^{6} y^{5}+d y^{25}\end{array}$ & $\begin{array}{c}t+z^{3}+g\left(z, x, y^{1 / 5}\right) \subset \mathbb{P}(15,5,2,3) \\
\mathbb{P}(5,2,3), \text { Diff }=\left(\frac{1}{2}, 0,0, \frac{4}{5}\right)\end{array}$ & 6 \\
\hline 256 & $\begin{array}{c}z x^{5}+x y^{n} \\
n=7 ; 9 ; 11 ; 15 ; 17 \\
19,21 ; 23,25\end{array}$ & $\begin{array}{l}t+z^{3}+z x^{5}+x y \subset \mathbb{P}(15,5,2,13) \\
\mathbb{P}(5,2,13), \text { Diff }=\left(\frac{1}{2}, 0,0, \frac{n-1}{n}\right)\end{array}$ & $\begin{array}{l}7,10,11, \\
16,18,22, \\
26\end{array}$ \\
\hline 257 & $\begin{array}{c}z x^{5}+x y^{2 n}, n=4,5 ; 6 \\
7,8,9 ; 10,11,12\end{array}$ & $\begin{array}{c}t^{2}+z^{3}+z x^{5}+x y^{2} \subset \mathbb{P}(15,10,4,13) \\
\text { Diff }=\left(0,0,0, \frac{n-1}{n}\right)\end{array}$ & $5,9,13$ \\
\hline 258 & $\begin{array}{c}z x^{5}+x^{2} y^{2 n} \\
n=4,5,6,7 ; 8,9,10\end{array}$ & $\begin{array}{c}t^{2}+z^{3}+z x^{5}+x^{2} y^{2} \subset \mathbb{P}(15,10,4,11) \\
\text { Diff }=\left(0,0,0, \frac{n-1}{n}\right)\end{array}$ & 7,11 \\
\hline 259 & $\begin{array}{c}z x^{5}+x^{2} y^{n}, n=7 ; 9 \\
13 ; 15,17 ; 19,21\end{array}$ & $\begin{array}{c}t+z^{3}+z x^{5}+x^{2} y \subset \mathbb{P}(15,5,2,11) \\
\mathbb{P}(5,2,11), \text { Diff }=\left(\frac{1}{2}, 0,0, \frac{n-1}{n}\right)\end{array}$ & $\begin{array}{c}7,10,14 \\
18,22\end{array}$ \\
\hline 260 & $\begin{array}{c}z x^{5}+x^{3} y^{n} \\
n=7 ; 11,13 ; 17\end{array}$ & $\begin{array}{c}t+z^{3}+z x^{5}+x^{3} y \subset \mathbb{P}(15,5,2,9) \\
\mathbb{P}(5,2,9), \text { Diff }=\left(\frac{1}{2}, 0,0, \frac{n-1}{n}\right)\end{array}$ & $8,14,18$ \\
\hline 261 & $\begin{array}{l}z x^{5}+x^{3} y^{2 n} \\
n=4,5 ; 7,8\end{array}$ & $\begin{array}{c}t^{2}+z^{3}+z x^{5}+x^{3} y^{2} \subset \mathbb{P}(15,10,4,9) \\
\text { Diff }=\left(0,0,0, \frac{n-1}{n}\right)\end{array}$ & 5,9 \\
\hline 262 & $\begin{array}{c}z x^{5}+x^{4} y^{2 n} \\
n=4,5,6\end{array}$ & $\begin{array}{c}t^{2}+z^{3}+z x^{5}+x^{4} y^{2} \subset \mathbb{P}(15,10,4,7) \\
\text { Diff }=\left(0,0,0, \frac{n-1}{n}\right)\end{array}$ & 7 \\
\hline 263 & $\begin{array}{l}z x^{5}+x^{4} y^{n} \\
n=9 ; 11,13\end{array}$ & $\begin{array}{c}t+z^{3}+z x^{5}+x^{4} y \subset \mathbb{P}(15,5,2,7) \\
\mathbb{P}(5,2,7), \text { Diff }=\left(\frac{1}{2}, 0,0, \frac{n-1}{n}\right)\end{array}$ & 10,14 \\
\hline 264 & $\begin{array}{c}z x^{5} y+y^{2 n} \\
n=5 ; 8 ; 10,11\end{array}$ & $\begin{array}{c}t^{2}+z^{3}+z x y+y^{2 n} \subset \mathbb{P}(3 n, 2 n, 4 n-3,3) \\
\text { Diff }=\left(0,0, \frac{4}{5}, 0\right)\end{array}$ & $7,11,15$ \\
\hline 265 & $\begin{array}{c}z x^{5} y+y^{n} \\
n=11 ; 13 ; 17 ; 23\end{array}$ & $\begin{array}{c}t+z^{3}+z x y+y^{n} \subset \mathbb{P}(3 n, n, 2 n-3,3) \\
\mathbb{P}(n, 2 n-3,3), \text { Diff }=\left(\frac{1}{2}, 0, \frac{4}{5}, 0\right)\end{array}$ & $\begin{array}{c}14,16,22, \\
30\end{array}$ \\
\hline
\end{tabular}


Продолжение таблицы 1

\begin{tabular}{|c|c|c|c|}
\hline 266 & $\begin{array}{c}z\left(x^{5} y+a y^{4 n}\right)+b x^{5} y^{2 n+1}+ \\
+c y^{6 n}, n=2|a|+|c| \neq 0,3\end{array}$ & $\begin{array}{c}t^{2}+z^{3}+g\left(z, x^{1 / 5}, y\right) \subset \mathbb{P}(3 n, 2 n, 4 n-1,1) \\
\text { Diff }=\left(0,0, \frac{4}{5}, 0\right)\end{array}$ & 5 \\
\hline 267 & $\begin{array}{l}z\left(x^{5} y+a y^{2 n}\right)+b x^{5} y^{n+1}+ \\
+c y^{3 n}, n=5|a|+|c| \neq 0 ; 7\end{array}$ & $\begin{array}{c}t+z^{3}+g\left(z, x^{1 / 5}, y\right) \subset \mathbb{P}(3 n, n, 2 n-1,1) \\
\mathbb{P}(n, 2 n-1,1), \text { Diff }=\left(\frac{1}{2}, 0, \frac{4}{5}, 0\right)\end{array}$ & 6,10 \\
\hline 268 & $\begin{array}{l}z y x^{2+3 i} f_{1-i}\left(x^{3}, y^{5}\right)+ \\
+a y^{4} x^{3 j} f_{2-j}\left(x^{3}, y^{5}\right)\end{array}$ & $\begin{array}{c}t^{2}+z^{3}+g(z, x, y) \subset \mathbb{P}(21,14,5,3) \\
\text { Diff }=\varnothing\end{array}$ & 3 \\
\hline 269 & $\begin{array}{l}z y x^{2+3 i} f_{1-i}\left(x^{3}, y^{7}\right)+ \\
+a y^{5} x^{3 j} f_{2-j}\left(x^{3}, y^{7}\right)\end{array}$ & $\begin{array}{c}t+z^{3}+g(z, x, y) \subset \mathbb{P}(57,19,7,3) \\
\mathbb{P}(19,7,3), \text { Diff }=\left(\frac{1}{2}, 0,0,0\right)\end{array}$ & 6 \\
\hline 270 & $\begin{array}{l}z x^{5} y+x y^{2 n+1} \\
n=4 ; 5,6,7 ; 8,9\end{array}$ & $\begin{array}{c}t^{2}+z^{3}+g(z, x, y) \subset \mathbb{P}(15 n+6,10 n+4,4 n-1,13) \\
\text { Diff }=\varnothing\end{array}$ & $5,9,13$ \\
\hline 271 & $\begin{array}{c}z x^{5} y+x y^{n}, n=10,12 \\
14 ; 16,18 ; 20\end{array}$ & $\begin{array}{c}t+z^{3}+g(z, x, y) \subset \mathbb{P}(15 n-3,5 n-1,2 n-3,13) \\
\mathbb{P}(5 n-1,2 n-3,13), \text { Diff }=\left(\frac{1}{2}, 0,0,0\right)\end{array}$ & $\begin{array}{c}14,18,22 \\
26\end{array}$ \\
\hline 272 & $\begin{array}{l}z x^{5} y+x^{2} y^{2 n} \\
n=4,5,6 ; 7,8\end{array}$ & $\begin{array}{c}t^{2}+z^{3}+g(z, x, y) \subset \mathbb{P}(15 n-3,10 n-2,4 n-3,11) \\
\text { Diff }=\varnothing\end{array}$ & 7,11 \\
\hline 273 & $\begin{array}{c}\quad z x^{5} y+x^{2} y^{n} \\
n=9 ; 11 ; 13,15 ; 17\end{array}$ & $\begin{array}{c}t+z^{3}+g(z, x, y) \subset \mathbb{P}(15 n-6,5 n-2,2 n-3,11) \\
\mathbb{P}(5 n-2,2 n-3,11), \text { Diff }=\left(\frac{1}{2}, 0,0,0\right)\end{array}$ & $\begin{array}{l}10,14,18 \\
22\end{array}$ \\
\hline 274 & $\begin{array}{c}z x^{5} y+x^{3} y^{2 n+1} \\
n=3 ; 5,6\end{array}$ & $\begin{array}{c}t^{2}+z^{3}+g(z, x, y) \subset \mathbb{P}(15 n+3,10 n+2,4 n-1,9) \\
\text { Diff }=\varnothing\end{array}$ & 5,9 \\
\hline 275 & $\begin{array}{c}z x^{5} y+x^{3} y^{n} \\
n=8 ; 10 ; 14\end{array}$ & $\begin{array}{c}t+z^{3}+g(z, x, y) \subset \mathbb{P}(15 n-9,5 n-3,2 n-3,9) \\
\mathbb{P}(5 n-3,2 n-3,9), \text { Diff }=\left(\frac{1}{2}, 0,0,0\right)\end{array}$ & $10,14,18$ \\
\hline 276 & $\begin{array}{c}z x^{5} y+x^{4} y^{2 n} \\
n=4,5\end{array}$ & $\begin{array}{c}t^{2}+z^{3}+g(z, x, y) \subset \mathbb{P}(15 n-6,10 n-4,4 n-3,7) \\
\text { Diff }=\varnothing\end{array}$ & 7 \\
\hline 277 & $\begin{array}{c}z x^{5} y+x^{4} y^{n} \\
n=7,9 ; 11\end{array}$ & $\begin{array}{c}t+z^{3}+g(z, x, y) \subset \mathbb{P}(15 n-12,5 n-4,2 n-3,7) \\
\mathbb{P}(5 n-4,2 n-3,7), \text { Diff }=\left(\frac{1}{2}, 0,0,0\right)\end{array}$ & 10,14 \\
\hline 278 & $\begin{array}{l}z x^{7}+y^{n} \\
n=11,13\end{array}$ & $\begin{array}{c}t+z^{3}+z x+y \subset \mathbb{P}(3,1,2,3) \\
\mathbb{P}(1,2,3), \text { Diff }=\left(\frac{1}{2}, 0, \frac{6}{7}, \frac{n-1}{n}\right)\end{array}$ & 14 \\
\hline 279 & $\begin{array}{c}z x^{7}+x y^{2 n} \\
n=5 ; 6\end{array}$ & $\begin{array}{c}t^{2}+z^{3}+z x^{7}+x y^{2} \subset \mathbb{P}(21,14,4,19) \\
\text { Diff }=\left(0,0,0, \frac{n-1}{n}\right)\end{array}$ & 5,19 \\
\hline 280 & $z x^{7}+x y^{11}$ & $\begin{array}{c}t+z^{3}+z x^{7}+x y \subset \mathbb{P}(21,7,2,19) \\
\mathbb{P}(7,2,19), \text { Diff }=\left(\frac{1}{2}, 0,0, \frac{10}{11}\right)\end{array}$ & 24 \\
\hline 281 & $\begin{array}{c}z x^{7}+x^{2} y^{n} \\
n=9 ; 11\end{array}$ & $\begin{array}{c}t+z^{3}+z x^{7}+x^{2} y \subset \mathbb{P}(21,7,2,17) \\
\mathbb{P}(7,2,17), \text { Diff }=\left(\frac{1}{2}, 0,0, \frac{n-1}{n}\right)\end{array}$ & 10,34 \\
\hline 282 & $z x^{7}+x^{2} y^{10}$ & $\begin{array}{c}t^{2}+z^{3}+z x^{7}+x^{2} y^{2} \subset \mathbb{P}(21,14,4,17) \\
\text { Diff }=\left(0,0,0, \frac{4}{5}\right)\end{array}$ & 10 \\
\hline 283 & $z x^{7}+x^{3} y^{8}$ & $\begin{array}{c}t^{2}+z^{3}+z x^{7}+x^{3} y^{2} \subset \mathbb{P}(21,14,4,15) \\
\text { Diff }=\left(0,0,0, \frac{3}{4}\right)\end{array}$ & 5 \\
\hline 284 & $z x^{7}+x^{4} y^{7}$ & $\begin{array}{c}t+z^{3}+z x^{7}+x^{4} y \subset \mathbb{P}(21,7,2,13) \\
\mathbb{P}(7,2,13), \text { Diff }=\left(\frac{1}{2}, 0,0, \frac{6}{7}\right)\end{array}$ & 16 \\
\hline
\end{tabular}


Продолжение таблицы 1

\begin{tabular}{|c|c|c|c|}
\hline 285 & $z x^{7}+x^{4} y^{8}$ & $\begin{array}{c}t^{2}+z^{3}+z x^{7}+x^{4} y^{2} \subset \mathbb{P}(21,14,4,13) \\
\text { Diff }=\left(0,0,0, \frac{3}{4}\right)\end{array}$ & 13 \\
\hline 286 & $z x^{7}+x^{5} y^{7}$ & $\begin{array}{c}t+z^{3}+z x^{7}+x^{5} y \subset \mathbb{P}(21,7,2,11) \\
\mathbb{P}(7,2,11), \text { Diff }=\left(\frac{1}{2}, 0,0, \frac{6}{7}\right)\end{array}$ & 22 \\
\hline 287 & $\begin{array}{l}z x^{6} y+y^{n} \\
n=11,13\end{array}$ & $\begin{array}{c}t^{2}+z^{3}+z x^{2} y+y^{n} \subset \mathbb{P}(3 n, 2 n, 2 n-3,6) \\
\text { Diff }=\left(0,0, \frac{2}{3}, 0\right)\end{array}$ & 9 \\
\hline 288 & $z x^{6} y+y^{14}$ & $\begin{array}{c}t^{2}+z^{3}+z x y+y^{14} \subset \mathbb{P}(21,14,25,3) \\
\text { Diff }=\left(0,0, \frac{5}{6}, 0\right)\end{array}$ & 18 \\
\hline 289 & $\begin{array}{l}z\left(x^{6} y+a y^{8}\right)+b x^{6} y^{5}+ \\
\quad+c y^{12},|a|+|c| \neq 0\end{array}$ & $\begin{array}{c}t^{2}+z^{3}+f\left(z, x^{1 / 6}, y\right) \subset \mathbb{P}(6,4,7,1) \\
\text { Diff }=\left(0,0, \frac{5}{6}, 0\right)\end{array}$ & 6 \\
\hline 290 & $\begin{array}{c}z x^{6} y+x y^{n} \\
n=10,11,12 ; 13\end{array}$ & $\begin{array}{c}t+z^{3}+z x^{6} y+x y^{n} \subset \mathbb{P}(18 n-3,6 n-1,2 n-3,16) \\
\mathbb{P}(6 n-1,2 n-3,16), \text { Diff }=\left(\frac{1}{2}, 0,0,0\right)\end{array}$ & 14,32 \\
\hline 291 & $\begin{array}{c}z x^{6} y+x^{2} y^{2 n+1} \\
n=4 ; 5\end{array}$ & $\begin{array}{c}t^{2}+z^{3}+z x^{3} y+x y^{2 n+1} \subset \mathbb{P}(9 n+3,6 n+2,4 n-1,7) \\
\text { Diff }=\left(0,0, \frac{1}{2}, 0\right)\end{array}$ & 5,14 \\
\hline 292 & $z x^{6} y+x^{2} y^{10}$ & $\begin{array}{c}t^{2}+z^{3}+z x^{6} y+x^{2} y^{10} \subset \mathbb{P}(87,58,17,14) \\
\text { Diff }=\varnothing\end{array}$ & 7 \\
\hline 293 & $\begin{array}{c}z x^{6} y+x^{3} y^{n} \\
n=8 ; 10\end{array}$ & $\begin{array}{c}t+z^{3}+z x^{2} y+x y^{n} \subset \mathbb{P}(6 n-3,2 n-1,2 n-3,4) \\
\mathbb{P}(2 n-1,2 n-3,4), \text { Diff }=\left(\frac{1}{2}, 0, \frac{2}{3}, 0\right)\end{array}$ & 10,24 \\
\hline 294 & $z x^{6} y+x^{4} y^{7}$ & $\begin{array}{c}t^{2}+z^{3}+z x^{6} y+x^{4} y^{7} \subset \mathbb{P}(57,38,11,10) \\
\text { Diff }=\varnothing\end{array}$ & 5 \\
\hline 295 & $z x^{6} y+x^{4} y^{8}$ & $\begin{array}{c}t^{2}+z^{3}+z x^{3} y+x^{2} y^{8} \subset \mathbb{P}(33,22,13,5) \\
\text { Diff }=\left(0,0, \frac{1}{2}, 0\right)\end{array}$ & 10 \\
\hline 296 & $z x^{6} y+x^{5} y^{7}$ & $\begin{array}{c}t+z^{3}+g(z, x, y) \subset \mathbb{P}(111,37,11,8) \\
\mathbb{P}(37,11,8), \text { Diff }=\left(\frac{1}{2}, 0,0,0\right)\end{array}$ & 16 \\
\hline 297 & $\begin{array}{c}z x^{5} y^{2}+y^{n} \\
n=11 ; 17\end{array}$ & $\begin{array}{c}t+z^{3}+z x y^{2}+y^{n} \subset \mathbb{P}(3 n, n, 2 n-6,3) \\
\mathbb{P}(n, 2 n-6,3), \text { Diff }=\left(\frac{1}{2}, 0, \frac{4}{5}, 0\right)\end{array}$ & 18,30 \\
\hline 298 & $z x^{5} y^{2}+y^{14}$ & $\begin{array}{c}t^{2}+z^{3}+z x y^{2}+y^{14} \subset \mathbb{P}(21,14,22,3) \\
\text { Diff }=\left(0,0, \frac{4}{5}, 0\right)\end{array}$ & 11 \\
\hline 299 & $z x^{5} y^{2}+y^{16}$ & $\begin{array}{c}t^{2}+z^{3}+z x y+y^{8} \subset \mathbb{P}(12,8,13,3) \\
\text { Diff }=\left(0,0, \frac{4}{5}, \frac{1}{2}\right)\end{array}$ & 15 \\
\hline 300 & $\begin{array}{c}z\left(x^{5} y^{2}+a y^{10}\right)+b x^{5} y^{7}+ \\
+c y^{15}\end{array}$ & $\begin{array}{c}t+z^{3}+g\left(z, x^{1 / 5}, y\right) \subset \mathbb{P}(15,5,8,1) \\
\mathbb{P}(5,8,1), \text { Diff }=\left(\frac{1}{2}, 0, \frac{4}{5}, 0\right)\end{array}$ & 10 \\
\hline 301 & $\begin{array}{c}z x^{5} y^{2}+x y^{4 n+2} \\
n=2 ; 3\end{array}$ & $\begin{array}{c}t^{2}+z^{3}+g\left(z, x, y^{1 / 2}\right) \subset \mathbb{P}(15 n+6,10 n+4,4 n-1,13) \\
\text { Diff }=\left(0,0,0, \frac{1}{2}\right)\end{array}$ & 9,13 \\
\hline 302 & $\begin{array}{l}z x^{5} y^{2}+x y^{n} \\
n=11 ; 13 ; 15\end{array}$ & $\begin{array}{c}t+z^{3}+z x^{5} y^{2}+x y^{n} \subset \mathbb{P}(15 n-6,5 n-2,2 n-6,13) \\
\mathbb{P}(5 n, 2 n-6,13), \text { Diff }=\left(\frac{1}{2}, 0,0,0\right)\end{array}$ & $18,22,26$ \\
\hline 303 & $z x^{5} y^{2}+x y^{12}$ & $\begin{array}{c}t^{2}+z^{3}+z x^{5} y^{2}+x y^{12} \subset \mathbb{P}(87,58,18,13) \\
\text { Diff }=\varnothing\end{array}$ & 9 \\
\hline
\end{tabular}


Продолжение таблицы 1

\begin{tabular}{|c|c|c|c|}
\hline 304 & $z x^{5} y^{2}+x^{2} y^{n}$ & $t+z^{3}+g(z, x, y) \subset \mathbb{P}(15 n-12,5 n-4,2 n-6,11)$ & $14,18,22$ \\
& $n=9 ; 11 ; 13$ & $\mathbb{P}(5 n-4,2 n-6,11)$, Diff $=\left(\frac{1}{2}, 0,0,0\right)$ & \\
\hline 305 & $z x^{5} y^{2}+x^{2} y^{10}$ & $t^{2}+z^{3}+z x^{5} y^{2}+x^{2} y^{10} \subset \mathbb{P}(69,46,14,11)$ & 7 \\
& & $\operatorname{Diff}=\varnothing$ & 11 \\
\hline 306 & $z x^{5} y^{2}+x^{2} y^{12}$ & $t^{2}+z^{3}+z x^{5} y+x^{2} y^{6} \subset \mathbb{P}(42,28,9,11)$ & \\
\hline 307 & $z x^{5} y^{2}+x^{3} y^{8}$ & $t^{2}+z^{3}+z x^{5} y^{2}+x^{3} y^{8} \subset \mathbb{P}(51,34,10,9)$ & 5 \\
\hline 308 & $z x^{5} y^{2}+x^{3} y^{10}$ & $t^{2}+z^{3}+z x^{5} y+x^{3} y^{5} \subset \mathbb{P}(33,22,7,9)$ & 9 \\
\hline 309 & $z x^{5} y^{2}+x^{3} y^{11}$ & $\operatorname{Diff}=\left(0,0,0, \frac{1}{2}\right)$ & 18 \\
& & $t+z^{3}+g(z, x, y) \subset \mathbb{P}(147,49,16,9)$ & 10,14 \\
\hline 310 & $z x^{5} y^{2}+x^{4} y^{n}$ & $\mathbb{P}(49,16,9), \operatorname{Diff}=\left(\frac{1}{2}, 0,0,0\right)$ & \\
\hline 311 & $n=7 ; 9$ & $\mathbb{P}(5 n-8,2 n-6,7), \operatorname{Diff}=\left(\frac{1}{2}, 0,0,0\right)$ & \\
\hline & $z x^{5} y^{2}+x^{4} y^{8}$ & $t^{2}+z^{3}+z x^{5} y+x^{4} y^{4} \subset \mathbb{P}(24,16,5,7)$ & \\
\hline
\end{tabular}

ТАБЛИЦА 2. Особенность: $t^{2}+z^{4}+g(z, x, y)$

\begin{tabular}{|c|c|c|c|}
\hline 1 & $a z^{2} y^{n}+x^{5}+b y^{2 n}$ & $t^{2}+z^{2}+a z y+x+b y^{2} \subset \mathbb{P}(1,1,2,1)$ & 5,10 \\
& $n=3 ; 7,9$ & $\mathbb{P}^{2}$, Diff $=\left(0, \frac{1}{2}, \frac{4}{5}, \frac{n-1}{n}\right)$ & \\
\hline 2 & $x^{5}+y^{n}$ & $t^{2}+z^{2}+x+y \subset \mathbb{P}(1,1,2,2)$ & $7,10,11$, \\
& $n=7 ; 9 ; 11 ; 13 ; 17,19$ & $\mathbb{P}(1,1,2)$, Diff $=\left(0, \frac{1}{2}, \frac{4}{5}, \frac{n-1}{n}\right)$ & 15,20 \\
\hline 3 & $a z^{2} y^{2 n}+b z y^{3 n}+$ & $t^{2}+z^{4}+g\left(z, x^{1 / 5}, y^{1 / n}\right) \subset \mathbb{P}(2,1,4,1)$ & 5 \\
& $+c y^{4 n}+x^{5}, n=2,3,4$ & $\mathbb{P}(2,1,1)$, Diff $=\left(0,0, \frac{4}{5}, \frac{n-1}{n}\right)$ & \\
\hline 4 & $a z^{2} y x^{i} f_{2-i}\left(x, y^{2}\right)+$ & $t^{2}+z^{2}+g\left(z^{1 / 2}, x, y\right) \subset \mathbb{P}(5,5,2,1)$ & 2 \\
& $+x^{j} f_{5-j}\left(x, y^{2}\right), i \leqslant 1|| j \leqslant 2$ & $\operatorname{Diff}=\left(0, \frac{1}{2}, 0,0\right)$ & 4 \\
\hline 5 & $x^{i} f_{5-i}\left(x, y^{3}\right)$ & $t^{2}+z^{2}+x^{i} f_{5-i}(x, y) \subset \mathbb{P}(5,5,2,2)$ & $6,7,11$, \\
& $i=1,2,3$ & $\operatorname{Diff}=\left(0, \frac{1}{2}, 0, \frac{2}{3}\right)$ & 16 \\
\hline 6 & $x^{5}+x y^{n}$ & $t^{2}+z^{2}+x^{5}+x y \subset \mathbb{P}(5,5,2,8)$ & 3,8 \\
& $n=5 ; 7 ; 9,11 ; 13,15$ & $\operatorname{Diff}=\left(0, \frac{1}{2}, 0, \frac{n-1}{n}\right)$ & \\
\hline 7 & $x^{5}+a x^{3} y^{n}+b x y^{2 n}$ & $t^{2}+z^{2}+x^{5}+a x^{3} y+b x y^{2} \subset \mathbb{P}(5,5,2,4)$ & 2,3 \\
& $n=3 b \neq 0 ; 5,7$ & $\operatorname{Diff}=\left(0, \frac{1}{2}, 0, \frac{n-1}{n}\right)$ & \\
\hline 8 & $a z^{2} x y^{2 n}+z\left(b x^{3} y^{n}+c y^{5 n}\right)+$ & $t^{2}+z^{4}+g\left(z, x, y^{1 / n}\right) \subset \mathbb{P}(10,5,4,3)$ & \\
& $+x^{5}+e x^{2} y^{4 n}, n=1 c \neq 0 ; 2$ & $\operatorname{Diff}=\left(0,0,0, \frac{n-1}{n}\right)$ & \\
& $|a|+|c|+|e| \neq 0$ & & \\
\hline 9 & $x^{5}+x^{2} y^{n}$ & $t^{2}+z^{2}+x^{5}+x^{2} y \subset \mathbb{P}(5,5,2,6)$ & \\
& $n=5,7 ; 11$ & $\operatorname{Diff}=\left(0, \frac{1}{2}, 0, \frac{n-1}{n}\right)$ & \\
\hline
\end{tabular}


Продолжение таблицы 2

\begin{tabular}{|c|c|c|c|}
\hline 10 & $x^{5}+a z^{2} x y^{5}+b x^{2} y^{10}$ & $\begin{array}{c}t^{2}+z^{2}+x^{5}+a z x y+b x^{2} y^{2} \subset \mathbb{P}(5,5,2,3) \\
\text { Diff }=\left(0, \frac{1}{2}, 0, \frac{4}{5}\right)\end{array}$ & 6 \\
\hline 11 & $\begin{array}{c}x^{5}+z y^{n} \\
n=4 ; 7 ; 8 ; 11 ; 13,14\end{array}$ & $\begin{array}{c}t^{2}+z^{4}+x+z y \subset \mathbb{P}(2,1,4,3) \\
\mathbb{P}(2,1,3), \text { Diff }=\left(0,0, \frac{4}{5}, \frac{n-1}{n}\right)\end{array}$ & $\begin{array}{r}5,7,10 \\
11,15\end{array}$ \\
\hline 12 & $\begin{array}{c}x^{5}+z x y^{n}, n=4 \\
5,6 ; 7 ; 8,9,10\end{array}$ & $\begin{array}{c}t^{2}+z^{4}+x^{5}+z x y \subset \mathbb{P}(10,5,4,11) \\
\text { Diff }=\left(0,0,0, \frac{n-1}{n}\right)\end{array}$ & $6,7,11$ \\
\hline 13 & $\begin{array}{c}x^{5}+z x^{2} y^{n} \\
n=4,5,6\end{array}$ & $\begin{array}{c}t^{2}+z^{4}+x^{5}+z x^{2} y \subset \mathbb{P}(10,5,4,7) \\
\text { Diff }=\left(0,0,0, \frac{n-1}{n}\right)\end{array}$ & 7 \\
\hline 14 & $\begin{array}{l}\underline{a} z^{2} x^{i+1} f_{2-i}(x, y)+ \\
+\underline{b} x^{j} f_{6-j}(x, y), j \leqslant 1 \\
\end{array}$ & $\begin{array}{c}t^{2}+z^{2}+g\left(z^{1 / 2}, x, y\right) \subset \mathbb{P}(3,3,1,1) \\
\text { Diff }=\left(0, \frac{1}{2}, 0,0\right)\end{array}$ & 2 \\
\hline 15 & $\begin{array}{c}a z^{2} x^{3}+b x^{6}+y^{n} \\
n=7 ; 11\end{array}$ & $\begin{array}{c}t^{2}+z^{2}+a z x+b x^{2}+y \subset \mathbb{P}(1,1,1,2) \\
\mathbb{P}^{2}, \text { Diff }=\left(0, \frac{1}{2}, \frac{2}{3}, \frac{n-1}{n}\right)\end{array}$ & 7,12 \\
\hline $\begin{array}{l}16 \\
(1)\end{array}$ & $\begin{array}{c}z^{3} y^{2}+z^{2}\left(x^{3}+y^{4}\right)+z y^{2}\left(x^{3}+\right. \\
\left.+y^{4}\right)+x^{6}+x^{3} y^{4}+y^{8}\end{array}$ & $\begin{array}{c}t^{2}+z^{4}+g\left(z, x^{1 / 3}, y^{1 / 2}\right) \subset \mathbb{P}(2,1,2,1) \\
\text { Diff }=\left(0,0, \frac{2}{3}, \frac{1}{2}\right)\end{array}$ & 3 \\
\hline 17 & $\begin{array}{c}\underline{a} z^{2} x^{2 i+1} f_{1-i}\left(x^{2}, y^{3}\right)+ \\
\underline{b} x^{2 j} f_{3-j}\left(x^{2}, y^{3}\right)\end{array}$ & $\begin{array}{c}t^{2}+z^{2}+g\left(z^{1 / 2}, x, y^{1 / 3}\right) \subset \mathbb{P}(3,3,1,2) \\
\text { Diff }=\left(0, \frac{1}{2}, 0, \frac{2}{3}\right)\end{array}$ & 4 \\
\hline 18 & $\begin{array}{c}\underline{a} z^{2} x^{3 i} f_{1-i}\left(x^{3}, y^{5}\right)+ \\
\underline{b} x^{3 j} f_{2-j}\left(x^{3}, y^{5}\right)\end{array}$ & $\begin{array}{c}t^{2}+z^{2}+g\left(z^{1 / 2}, x^{1 / 3}, y^{1 / 5}\right) \subset \mathbb{P}(1,1,1,1) \\
\text { Diff }=\left(0, \frac{1}{2}, \frac{2}{3}, \frac{4}{5}\right)\end{array}$ & 6 \\
\hline 19 & $\begin{array}{c}a z^{2} x^{3}+b z x^{2} y^{n}+c x^{6}+ \\
+d x y^{2 n}, n=3 \& d \neq 0 \\
n=4 \&|b|+|d| \neq 0\end{array}$ & $\begin{array}{c}t^{2}+z^{4}+g\left(z, x, y^{1 / n}\right) \subset \mathbb{P}(6,3,2,5) \\
\operatorname{Diff}=\left(0,0,0, \frac{n-1}{n}\right)\end{array}$ & 3,5 \\
\hline 20 & $\begin{array}{c}a z^{2} x^{3}+b x^{6}+x y^{n} \\
n=7 ; 9\end{array}$ & $\begin{array}{c}t^{2}+z^{2}+g\left(z^{1 / 2}, x, y^{1 / n}\right) \subset \mathbb{P}(3,3,1,5) \\
\text { Diff }=\left(0, \frac{1}{2}, 0, \frac{n-1}{n}\right)\end{array}$ & 7,10 \\
\hline 21 & $\begin{array}{c}a z^{2} x^{3}+b x^{6}+x^{2} y^{n} \\
n=5 ; 7\end{array}$ & $\begin{array}{c}t^{2}+z^{2}+g\left(z^{1 / 2}, x, y^{1 / n}\right) \subset \mathbb{P}(3,3,1,4) \\
\operatorname{Diff}=\left(0, \frac{1}{2}, 0, \frac{n-1}{n}\right)\end{array}$ & 5,8 \\
\hline 22 & $\begin{array}{c}a z^{2} x^{3}+z y^{n}+b x^{6} \\
n=5 ; 7 ; 8\end{array}$ & $\begin{array}{c}t^{2}+z^{4}+g\left(z, x^{1 / 3}, y^{1 / n}\right) \subset \mathbb{P}(2,1,2,3) \\
\text { Diff }=\left(0,0, \frac{2}{3}, \frac{n-1}{n}\right)\end{array}$ & $5,7,9$ \\
\hline 23 & $\begin{array}{c}a z^{2} x^{3}+z x y^{n}+c x^{6} \\
n=4 ; 5 ; 6\end{array}$ & $\begin{array}{c}t^{2}+z^{4}+g\left(z, x, y^{1 / n}\right) \subset \mathbb{P}(6,3,2,7) \\
\operatorname{Diff}=\left(0,0,0, \frac{n-1}{n}\right)\end{array}$ & $4,5,7$ \\
\hline 24 & $\begin{array}{c}x^{5} y+y^{n} \\
n=7 ; 9 ; 13 ; 15\end{array}$ & $\begin{array}{c}t^{2}+z^{2}+x y+y^{n} \subset \mathbb{P}(n, n, 2 n-2,2) \\
\text { Diff }=\left(0, \frac{1}{2}, \frac{4}{5}, 0\right)\end{array}$ & $\begin{array}{c}8,11,16, \\
20\end{array}$ \\
\hline 25 & $\begin{array}{c}a z^{2} y^{2 n}+b z y^{3 n}+ \\
+x^{5} y+d y^{4 n}, n=2,3\end{array}$ & $\begin{array}{c}t^{2}+z^{4}+g\left(z, x^{1 / 5}, y\right) \subset \mathbb{P}(2 n, n, 4 n-1,1) \\
\text { Diff }=\left(0,0, \frac{4}{5}, 0\right)\end{array}$ & 5 \\
\hline 26 & $\begin{array}{c}a z^{2} y^{n}+x^{5} y+b y^{2 n} \\
n=5 ; 7\end{array}$ & $\begin{array}{c}t^{2}+z^{2}+a z y^{n}+x y+b y^{2 n} \subset \mathbb{P}(n, n, 2 n-1,1) \\
\text { Diff }=\left(0, \frac{1}{2}, \frac{4}{5}, 0\right)\end{array}$ & 6,10 \\
\hline 27 & $\begin{array}{c}y x^{i} f_{5-i}\left(x, y^{2}\right) \\
i \leqslant 3\end{array}$ & $\begin{array}{c}t^{2}+z^{2}+g(x, y) \subset \mathbb{P}(11,11,4,2) \\
\operatorname{Diff}=\left(0, \frac{1}{2}, 0,0\right)\end{array}$ & 4 \\
\hline
\end{tabular}


Продолжение таблицы 2

\begin{tabular}{|c|c|c|c|}
\hline 28 & $\begin{array}{c}x^{5} y+x y^{n} \\
n=6 ; 8 ; 10 ; 12\end{array}$ & $\begin{array}{c}t^{2}+z^{2}+g(x, y) \subset \mathbb{P}(5 n-1,5 n-1,2 n-2,8) \\
\text { Diff }=\left(0, \frac{1}{2}, 0,0\right)\end{array}$ & $\begin{array}{c}7,11,12 \\
16\end{array}$ \\
\hline 29 & $\begin{array}{c}x^{5} y+a x^{3} y^{n+1}+b x y^{2 n+1} \\
n=3 \quad b \neq 0 ; 5\end{array}$ & $\begin{array}{c}t^{2}+z^{2}+g(x, y) \subset \mathbb{P}(5 n+2,5 n+2,2 n, 4) \\
\text { Diff }=\left(0, \frac{1}{2}, 0,0\right)\end{array}$ & 4,8 \\
\hline 30 & $\begin{array}{c}x^{5} y+x^{2} y^{n} \\
n=5 ; 9\end{array}$ & $\begin{array}{c}t^{2}+z^{2}+g(x, y) \subset \mathbb{P}(5 n-2,5 n-2,2 n-2,6) \\
\text { Diff }=\left(0, \frac{1}{2}, 0,0\right)\end{array}$ & 7,12 \\
\hline 31 & $\begin{array}{c}a z^{2} x y^{3}+z\left(b x^{3} y^{2}+c y^{7}\right)+ \\
+x^{5} y+e x^{2} y^{6},|a|+|c|+|e| \neq 0\end{array}$ & $\begin{array}{c}t^{2}+z^{4}+g(z, x, y) \subset \mathbb{P}(14,7,5,3) \\
\text { Diff }=\varnothing\end{array}$ & 3 \\
\hline 32 & $a z^{2} x y^{4}+x^{5} y+b x^{2} y^{8}$ & $\begin{array}{c}t^{2}+z^{2}+g\left(z^{1 / 2}, x, y\right) \subset \mathbb{P}(19,19,7,3) \\
\text { Diff }=\left(0, \frac{1}{2}, 0,0\right)\end{array}$ & 6 \\
\hline 33 & $\begin{array}{c}z y^{n}+x^{5} y \\
n=5 ; 8 ; 10,11\end{array}$ & $\begin{array}{c}t^{2}+z^{4}+g\left(z, x^{1 / 5}, y\right) \subset \mathbb{P}(2 n, n, 4 n-3,3) \\
\text { Diff }=\left(0,0, \frac{4}{5}, 0\right)\end{array}$ & $6,10,15$ \\
\hline 34 & $\begin{array}{l}z x y^{n}+x^{5} y \\
n=4,5 ; 6 ; 7,8\end{array}$ & $\begin{array}{c}t^{2}+z^{4}+g(z, x, y) \subset \mathbb{P}(10 n-2,5 n-1,4 n-3,11) \\
\text { Diff }=\varnothing\end{array}$ & $6,7,11$ \\
\hline 35 & $\begin{array}{c}z x^{2} y^{n}+x^{5} y \\
n=4,5\end{array}$ & $\begin{array}{c}t^{2}+z^{4}+g(z, x, y) \subset \mathbb{P}(10 n-4,5 n-2,4 n-3,7) \\
\text { Diff }=\varnothing\end{array}$ & 7 \\
\hline 36 & $\begin{array}{c}x^{i} y^{j} f_{7-i-j}(x, y) \\
i \leqslant 2 \& j \leqslant 2\end{array}$ & $\begin{array}{c}t^{2}+z^{2}+g(x, y) \subset \mathbb{P}(7,7,2,2) \\
\operatorname{Diff}=\left(0, \frac{1}{2}, 0,0\right)\end{array}$ & 4 \\
\hline 37 & $\begin{array}{c}z^{i} y^{2 j}\left(z+y^{2}\right)^{l} f_{4-i-j-l}\left(z, y^{2}\right)+ \\
+x^{7}, i \leqslant 2 \& j \leqslant 1 \& l \leqslant 2\end{array}$ & $\begin{array}{c}t^{2}+z^{4}+g\left(z, x^{1 / 7}, y^{1 / 2}\right) \subset \mathbb{P}(2,1,4,1) \\
\mathbb{P}(2,1,1), \text { Diff }=\left(0,0, \frac{6}{7}, \frac{1}{2}\right)\end{array}$ & 7 \\
\hline 38 & $x^{7}+y^{9}$ & $\begin{array}{c}t^{2}+z^{2}+x+y \subset \mathbb{P}(1,1,2,2) \\
\mathbb{P}(1,1,2), \text { Diff }=\left(0, \frac{1}{2}, \frac{6}{7}, \frac{8}{9}\right)\end{array}$ & 28 \\
\hline 39 & $x^{7}+x y^{7}$ & $\begin{array}{c}t^{2}+z^{2}+x^{7}+x y \subset \mathbb{P}(7,7,2,12) \\
\text { Diff }=\left(0, \frac{1}{2}, 0, \frac{6}{7}\right)\end{array}$ & 8 \\
\hline 40 & $a z^{2} x y^{3}+x^{7}+b x^{2} y^{6}$ & $\begin{array}{c}t^{2}+z^{2}+g\left(z^{1 / 2}, x, y^{1 / 3}\right) \subset \mathbb{P}(7,7,2,5) \\
\text { Diff }=\left(0, \frac{1}{2}, 0, \frac{2}{3}\right)\end{array}$ & 10 \\
\hline 41 & $x^{7}+x^{3} y^{5}$ & $\begin{array}{c}t^{2}+z^{2}+x^{7}+x^{3} y \subset \mathbb{P}(7,7,2,8) \\
\text { Diff }=\left(0, \frac{1}{2}, 0, \frac{4}{5}\right)\end{array}$ & 16 \\
\hline 42 & $z x y^{5}+x^{7}$ & $\begin{array}{c}t^{2}+z^{4}+x^{7}+z x y \subset \mathbb{P}(14,7,4,17) \\
\text { Diff }=\left(0,0,0, \frac{4}{5}\right)\end{array}$ & 10 \\
\hline 43 & $z x^{2} y^{4}+x^{7}$ & $\begin{array}{c}t^{2}+z^{4}+x^{7}+z x^{2} y \subset \mathbb{P}(14,7,4,13) \\
\text { Diff }=\left(0,0,0, \frac{3}{4}\right)\end{array}$ & 13 \\
\hline 44 & $\begin{array}{c}a z^{2} y^{4}+b z y^{6}+ \\
\quad+x^{6} y+c y^{8}\end{array}$ & $\begin{array}{c}t^{2}+z^{4}+g\left(z, x^{1 / 6}, y\right) \subset \mathbb{P}(4,2,7,1) \\
\mathbb{P}(2,1,1), \text { Diff }=\left(0,0, \frac{5}{6}, 0\right)\end{array}$ & 6 \\
\hline 45 & $x^{6} y+a x^{3} y^{5}+b y^{9}$ & $\begin{array}{c}t^{2}+z^{2}+g\left(x^{1 / 3}, y\right) \subset \mathbb{P}(9,9,8,2) \\
\text { Diff }=\left(0, \frac{1}{2}, \frac{2}{3}, 0\right)\end{array}$ & 12 \\
\hline 46 & $\begin{array}{c}x^{6} y+x y^{n} \\
n=7 ; 8\end{array}$ & $\begin{array}{c}t^{2}+z^{2}+x^{6} y+x y^{n} \subset \mathbb{P}(6 n-1,6 n-1,2 n-2,10) \\
\text { Diff }=\left(0, \frac{1}{2}, 0,0\right)\end{array}$ & 8,20 \\
\hline
\end{tabular}


Продолжение таблицы 2

\begin{tabular}{|c|c|c|c|}
\hline 47 & $a z^{2} x y^{3}+x^{6} y+b x^{2} y^{6}$ & $\begin{array}{c}t^{2}+z^{2}+g\left(z^{1 / 2}, x, y\right) \subset \mathbb{P}(17,17,5,4) \\
\text { Diff }=\left(0, \frac{1}{2}, 0,0\right)\end{array}$ & 8 \\
\hline 48 & $z y^{7}+x^{6} y$ & $\begin{array}{c}t^{2}+z^{4}+z y^{7}+x y \subset \mathbb{P}(14,7,25,3) \\
\text { Diff }=\left(0,0, \frac{5}{6}, 0\right)\end{array}$ & 18 \\
\hline 49 & $z x y^{5}+x^{6} y$ & $\begin{array}{c}t^{2}+z^{4}+z x y^{5}+x^{6} y \subset \mathbb{P}(58,29,17,14) \\
\text { Diff }=\varnothing\end{array}$ & 7 \\
\hline 50 & $z x^{2} y^{4}+x^{6} y$ & $\begin{array}{c}t^{2}+z^{4}+z x y^{4}+x^{3} y \subset \mathbb{P}(22,11,13,5) \\
\text { Diff }=\left(0,0, \frac{1}{2}, 0\right)\end{array}$ & 10 \\
\hline 51 & $\begin{array}{c}z^{i} y^{2 j} f_{4-i-j}\left(z, y^{2}\right)+ \\
+\left(z x^{5}|| \mid x^{5} y^{2}\right) \\
i \leqslant 2 \& j \leqslant 2 \&(j \leqslant 1|| \mid j=0)\end{array}$ & $\begin{array}{c}t^{2}+z^{4}+g\left(z, x^{1 / 5}, y^{1 / 2}\right) \subset \mathbb{P}(2,1,3,1) \\
\text { Diff }=\left(0,0, \frac{4}{5}, \frac{1}{2}\right)\end{array}$ & 5 \\
\hline 52 & $\begin{array}{c}x^{5} y^{2}+y^{n} \\
n=9 ; 11 \\
\end{array}$ & $\begin{array}{c}t^{2}+z^{2}+x y^{2}+y^{n} \subset \mathbb{P}(n, n, 2 n-4,2) \\
\text { Diff }=\left(0, \frac{1}{2}, \frac{4}{5}, 0\right)\end{array}$ & 15,20 \\
\hline 53 & $a z^{2} y^{5}+x^{5} y^{2}+b y^{10}$ & $\begin{array}{c}t^{2}+z^{2}+a z y^{5}+x y^{2}+b y^{10} \subset \mathbb{P}(5,5,8,1) \\
\text { Diff }=\left(0, \frac{1}{2}, \frac{4}{5}, 0\right)\end{array}$ & 10 \\
\hline 54 & $\begin{array}{c}x^{5} y^{2}+x y^{n} \\
n=7 ; 9\end{array}$ & $\begin{array}{c}t^{2}+z^{2}+x^{5} y^{2}+x y^{n} \subset \mathbb{P}(5 n-2,5 n-2,2 n-4,8) \\
\text { Diff }=\left(0, \frac{1}{2}, 0,0\right)\end{array}$ & 11,16 \\
\hline 55 & $x^{5} y^{2}+a x^{3} y^{5}+b x y^{8}$ & $\begin{array}{c}t^{2}+z^{2}+g\left(z^{1 / 2}, x, y\right) \subset \mathbb{P}(19,19,6,4) \\
\text { Diff }=\left(0, \frac{1}{2}, 0,0\right)\end{array}$ & 8 \\
\hline 56 & $a z^{2} x y^{3}+x^{5} y^{2}+b x^{2} y^{6}$ & $\begin{array}{c}t^{2}+z^{2}+g\left(z^{1 / 2}, x, y\right) \subset \mathbb{P}(13,13,4,3) \\
\text { Diff }=\left(0, \frac{1}{2}, 0,0\right)\end{array}$ & 6 \\
\hline 57 & $x^{5} y^{2}+x^{2} y^{7}$ & $\begin{array}{c}t^{2}+z^{2}+g\left(z^{1 / 2}, x, y\right) \subset \mathbb{P}(31,31,10,6) \\
\text { Diff }=\left(0, \frac{1}{2}, 0,0\right)\end{array}$ & 12 \\
\hline 58 & $z y^{7}+x^{5} y^{2}$ & $\begin{array}{c}t^{2}+z^{4}+z y^{7}+x y^{2} \subset \mathbb{P}(14,7,22,3) \\
\text { Diff }=\left(0,0, \frac{4}{5}, 0\right)\end{array}$ & 11 \\
\hline 59 & $z y^{8}+x^{5} y^{2}$ & $\begin{array}{c}t^{2}+z^{4}+z y^{4}+x y \subset \mathbb{P}(8,4,13,3) \\
\text { Diff }=\left(0,0, \frac{4}{5}, \frac{1}{2}\right)\end{array}$ & 15 \\
\hline 60 & $z x y^{5}+x^{5} y^{2}$ & $\begin{array}{c}t^{2}+z^{4}+g(z, x, y) \subset \mathbb{P}(46,23,14,11) \\
\text { Diff }=\varnothing\end{array}$ & 7 \\
\hline 61 & $z x y^{6}+x^{5} y^{2}$ & $\begin{array}{c}t^{2}+z^{4}+z x y^{3}+x^{5} y \subset \mathbb{P}(28,14,9,11) \\
\text { Diff }=\left(0,0,0, \frac{1}{2}\right)\end{array}$ & 11 \\
\hline 62 & $z x^{2} y^{4}+x^{5} y^{2}$ & $\begin{array}{c}t^{2}+z^{4}+z x^{2} y^{2}+x^{5} y \subset \mathbb{P}(16,8,5,7) \\
\operatorname{Diff}=\left(0,0,0, \frac{1}{2}\right)\end{array}$ & 7 \\
\hline 63 & $\begin{array}{c}a z^{2} y^{n}+z x^{4}+b y^{2 n} \\
n=3 \quad b \neq 0 ; 5 ; 7\end{array}$ & $\begin{array}{c}t^{2}+z^{4}+a z^{2} y+z x+b y^{2} \subset \mathbb{P}(2,1,3,2) \\
\text { Diff }=\left(0,0, \frac{3}{4}, \frac{n-1}{n}\right)\end{array}$ & $4,5,8$ \\
\hline 64 & $\begin{aligned} & z x^{4}+y^{n} \\
n= & 7 ; 9 ; 11 ; 13 ; 15\end{aligned}$ & $\begin{array}{c}t^{2}+z^{4}+z x+y \subset \mathbb{P}(2,1,3,4) \\
\mathbb{P}(2,1,3), \text { Diff }=\left(0,0, \frac{3}{4}, \frac{n-1}{n}\right)\end{array}$ & $\begin{array}{r}7,9,12 \\
13,16\end{array}$ \\
\hline
\end{tabular}


Продолжение таблицы 2

\begin{tabular}{|c|c|c|c|}
\hline 65 & $\begin{array}{c}a z^{2} y x^{2 i} f_{1-i}\left(x^{2}, y^{3}\right)+ \\
+z x^{2 j} f_{2-j}\left(x^{2}, y^{3}\right)+b y^{2} x^{2 l} \\
f_{2-l}\left(x^{2}, y^{3}\right), i=0|| j=0|| l \leqslant 1\end{array}$ & $\begin{array}{c}t^{2}+z^{4}+g\left(z, x^{1 / 2}, y\right) \subset \mathbb{P}(4,2,3,1) \\
\operatorname{Diff}=\left(0,0, \frac{1}{2}, 0\right)\end{array}$ & 2 \\
\hline 66 & $\begin{array}{c}a z^{2} y^{6}+z\left(x^{4}+b y^{9}\right)+ \\
+c x^{4} y^{3}+d y^{12},|a|+|b|+|d| \neq 0\end{array}$ & $\begin{array}{c}t^{2}+z^{4}+g\left(z, x^{1 / 4}, y^{1 / 3}\right) \subset \mathbb{P}(2,1,3,1) \\
\text { Diff }=\left(0,0, \frac{3}{4}, \frac{2}{3}\right)\end{array}$ & 4 \\
\hline 67 & $\begin{array}{c}z x^{4}+x y^{n}, n=5 ; 6 \\
7 ; 8,9 ; 10 ; 11,12\end{array}$ & $\begin{array}{c}t^{2}+z^{4}+z x^{4}+x y \subset \mathbb{P}(8,4,3,13) \\
\text { Diff }=\left(0,0,0, \frac{n-1}{n}\right)\end{array}$ & $\begin{array}{l}5,6,7 \\
9,10,13\end{array}$ \\
\hline 68 & $\begin{array}{c}a z^{2} x y^{n}+z x^{4}+b x^{2} y^{2 n} \\
n=3 \quad b \neq 0,4\end{array}$ & $\begin{array}{c}t^{2}+z^{4}+g\left(z, x, y^{1 / n}\right) \subset \mathbb{P}(8,4,3,5) \\
\text { Diff }=\left(0,0,0, \frac{n-1}{n}\right)\end{array}$ & 5 \\
\hline 69 & $\begin{array}{c}z x^{4}+x^{2} y^{n} \\
n=7 ; 9\end{array}$ & $\begin{array}{c}t^{2}+z^{4}+z x^{2}+x y \subset \mathbb{P}(4,2,3,5) \\
\text { Diff }=\left(0,0, \frac{1}{2}, \frac{n-1}{n}\right)\end{array}$ & 7,10 \\
\hline 70 & $\begin{array}{c}z x^{4}+x^{3} y^{n} \\
n=5,6\end{array}$ & $\begin{array}{c}t^{2}+z^{4}+z x^{4}+x^{3} y \subset \mathbb{P}(8,4,3,7) \\
\text { Diff }=\left(0,0,0, \frac{n-1}{n}\right)\end{array}$ & 7 \\
\hline 71 & $\begin{array}{c}z x^{5}+y^{n} \\
n=7 ; 9\end{array}$ & $\begin{array}{c}t^{2}+z^{4}+z x+y \subset \mathbb{P}(2,1,3,4) \\
\mathbb{P}(2,1,3), \text { Diff }=\left(0,0, \frac{4}{5}, \frac{n-1}{n}\right)\end{array}$ & 7,10 \\
\hline 72 & $\begin{array}{l}z x^{5}+x y^{n} \\
n=6 ; 7 ; 8\end{array}$ & $\begin{array}{c}t^{2}+z^{4}+z x^{5}+x y \subset \mathbb{P}(10,5,3,17) \\
\text { Diff }=\left(0,0,0, \frac{n-1}{n}\right)\end{array}$ & $6,7,17$ \\
\hline 73 & $z x^{5}+x^{2} y^{5}$ & $\begin{array}{c}t^{2}+z^{4}+z x^{5}+x^{2} y \subset \mathbb{P}(10,5,3,14) \\
\text { Diff }=\left(0,0,0, \frac{4}{5}\right)\end{array}$ & 7 \\
\hline 74 & $a z^{2} x y^{3}+z x^{5}+b x^{2} y^{6}$ & $\begin{array}{c}t^{2}+z^{4}+g\left(z, x, y^{1 / 3}\right) \subset \mathbb{P}(10,5,3,7) \\
\text { Diff }=\left(0,0,0, \frac{2}{3}\right)\end{array}$ & 7 \\
\hline 75 & $z x^{5}+x^{3} y^{5}$ & $\begin{array}{c}t^{2}+z^{4}+z x^{5}+x^{3} y \subset \mathbb{P}(10,5,3,11) \\
\operatorname{Diff}=\left(0,0,0, \frac{4}{5}\right)\end{array}$ & 11 \\
\hline 76 & $\begin{array}{l}z x^{4} y+y^{n} \\
n=7 ; 9 ; 11\end{array}$ & $\begin{array}{c}t^{2}+z^{4}+z x y+y^{n} \subset \mathbb{P}(2 n, n, 3 n-4,4) \\
\text { Diff }=\left(0,0, \frac{3}{4}, 0\right)\end{array}$ & $9,12,16$ \\
\hline 77 & $\begin{array}{c}a z^{2} y^{4}+z\left(x^{4} y+b y^{6}\right)+ \\
+c x^{4} y^{3}+d y^{8},|a|+|b|+|d| \neq 0\end{array}$ & $\begin{array}{c}t^{2}+z^{4}+g\left(z, x^{1 / 4}, y\right) \subset \mathbb{P}(4,2,5,1) \\
\text { Diff }=\left(0,0, \frac{3}{4}, 0\right)\end{array}$ & 4 \\
\hline 78 & $a z^{2} y^{5}+z x^{4} y+b y^{10}$ & $\begin{array}{c}t^{2}+z^{4}+g\left(z, x^{1 / 4}, y\right) \subset \mathbb{P}(10,5,13,2) \\
\text { Diff }=\left(0,0, \frac{3}{4}, 0\right)\end{array}$ & 8 \\
\hline 79 & $\begin{array}{l}z x^{4} y+x y^{n} \\
n=6 ; 7,8 ; 9\end{array}$ & $\begin{array}{c}t^{2}+z^{4}+g(z, x, y) \subset \mathbb{P}(8 n-2,4 n-1,3 n-4,13) \\
\text { Diff }=\varnothing\end{array}$ & $7,10,13$ \\
\hline 80 & $\begin{array}{c}z x^{4} y+x^{2} y^{n} \\
n=5 ; 7\end{array}$ & $\begin{array}{c}t^{2}+z^{4}+z x^{2} y+x y^{n} \subset \mathbb{P}(4 n-2,2 n-1,3 n-4,5) \\
\text { Diff }=\left(0,0, \frac{1}{2}, 0\right)\end{array}$ & 6,10 \\
\hline 81 & $a z^{2} x y^{3}+z x^{4} y+b x^{2} y^{6}$ & $\begin{array}{c}t^{2}+z^{4}+g(z, x, y) \subset \mathbb{P}(22,11,7,5) \\
\text { Diff }=\varnothing\end{array}$ & 5 \\
\hline 82 & $z x^{4} y+x^{3} y^{5}$ & $\begin{array}{c}t^{2}+z^{4}+g(z, x, y) \subset \mathbb{P}(34,17,11,7) \\
\text { Diff }=\varnothing\end{array}$ & 7 \\
\hline
\end{tabular}

(1) Имеем два случая. А) $t^{2}+z^{4}+z^{2}\left(a x^{3}+b y^{4}\right)+z y^{2}\left(c x^{3}+d y^{4}\right)+e x^{6}+k x^{3} y^{4}+$ $l y^{8}$. Тогда особенность исключительна, если выполняется следующее условие: $|b|+|d|+$ $|l| \neq 0 \&(e \neq 0||(a \neq 0 \&|c|+|k|+|l| \neq 0))$. 
В) $t^{2}+z^{3} y^{2}+z^{2} x^{3}+z y^{2}\left(a x^{3}+b y^{4}\right)+c x^{6}+d x^{3} y^{4}+e y^{8}$. Условие на исключительность: $|a b|+|c|+|d|+|e| \neq 0 \&|a|+|b|+|d|+|e| \neq 0$.

ТАБлИцА 3. Особенность: $t^{2}+z^{3} x+g(z, x, y)$

\begin{tabular}{|c|c|c|c|}
\hline 1 & $\begin{array}{c}a z x^{2} y^{2 n}+x^{5}+b y^{6 n} \\
n=2 \quad b \neq 0 ; 3,4\end{array}$ & $\begin{array}{c}t^{2}+z^{3} x+a z x^{2} y^{2}+x^{5}+b y^{6} \subset \mathbb{P}(15,8,6,5) \\
\text { Diff }=\left(0,0,0, \frac{n-1}{n}\right)\end{array}$ & 4,5 \\
\hline 2 & $\begin{array}{c}a z x^{2} y^{n}+x^{5}+b y^{3 n} \\
n=3 \quad b \neq 0 ; 7,9\end{array}$ & $\begin{array}{c}t+z^{3} x+a z x^{2} y+x^{5}+b y^{3} \subset \mathbb{P}(15,4,3,5) \\
\mathbb{P}(4,3,5), \text { Diff }=\left(\frac{1}{2}, 0,0, \frac{n-1}{n}\right)\end{array}$ & 4,10 \\
\hline 3 & $\begin{array}{c}x^{5}+y^{n} \\
n=7 ; 11 ; 13 ; 17 ; 19 ; \\
23 ; 29\end{array}$ & $\begin{array}{c}t+z x+x^{5}+y \subset \mathbb{P}(5,4,1,5) \\
\mathbb{P}(4,1,5), \operatorname{Diff}=\left(\frac{1}{2}, \frac{2}{3}, 0, \frac{n-1}{n}\right)\end{array}$ & $\begin{array}{c}7,12,14, \\
18,22,24, \\
30\end{array}$ \\
\hline 4 & $\begin{array}{c}x^{5}+y^{2 n} \\
n=4,7 ; 8 ; 11,13,14\end{array}$ & $\begin{array}{c}t^{2}+z x+x^{5}+y^{2} \subset \mathbb{P}(5,8,2,5) \\
\text { Diff }=\left(0, \frac{2}{3}, 0, \frac{n-1}{n}\right)\end{array}$ & $7,9,15$ \\
\hline 5 & $\begin{array}{c}x^{i}\left(x+y^{2}\right)^{j} f_{5-i-j}\left(x, y^{2}\right) \\
i \leqslant 1 \& j \leqslant 3\end{array}$ & $\begin{array}{c}t^{2}+z x+g(x, y) \subset \mathbb{P}(5,8,2,1) \\
\operatorname{Diff}=\left(0, \frac{2}{3}, 0,0\right)\end{array}$ & 3 \\
\hline (1) & $\begin{array}{c}z^{2} y f_{2}\left(x, y^{3}\right)+z y^{2} f_{3}\left(x, y^{3}\right)+ \\
+f_{5}\left(x, y^{3}\right)\end{array}$ & $\begin{array}{c}t+z^{3} x+g(z, x, y) \subset \mathbb{P}(15,4,3,1) \\
\mathbb{P}(4,3,1), \text { Diff }=\left(\frac{1}{2}, 0,0,0\right)\end{array}$ & 2 \\
\hline 7 & $\begin{array}{c}x^{i} f_{5-i}\left(x, y^{4}\right) \\
i \leqslant 3\end{array}$ & $\begin{array}{c}t^{2}+z x+g\left(x, y^{1 / 2}\right) \subset \mathbb{P}(5,8,2,1) \\
\text { Diff }=\left(0, \frac{2}{3}, 0, \frac{1}{2}\right)\end{array}$ & 3 \\
\hline 8 & $\begin{array}{c}x^{i} f_{5-i}\left(x, y^{5}\right) \\
i \leqslant 3\end{array}$ & $\begin{array}{c}t+z x+g\left(x, y^{1 / 5}\right) \subset \mathbb{P}(5,4,1,1) \\
\mathbb{P}(4,1,1), \text { Diff }=\left(\frac{1}{2}, \frac{2}{3}, 0, \frac{4}{5}\right)\end{array}$ & 6 \\
\hline 9 & $\begin{array}{l}x^{5}+x^{2} y^{2 n} \\
n=4,5,7,8\end{array}$ & $\begin{array}{c}t^{2}+z x+x^{5}+x^{2} y^{2} \subset \mathbb{P}(5,8,2,3) \\
\text { Diff }=\left(0, \frac{2}{3}, 0, \frac{n-1}{n}\right)\end{array}$ & 9 \\
\hline 10 & $\begin{array}{c}\quad x^{5}+x^{2} y^{n} \\
n=7 ; 11 ; 13,17\end{array}$ & $\begin{array}{l}t+z x+x^{5}+x^{2} y \subset \mathbb{P}(5,4,1,3) \\
\mathbb{P}(4,1,3), \text { Diff }=\left(\frac{1}{2}, \frac{2}{3}, 0, \frac{n-1}{n}\right)\end{array}$ & $10,12,18$ \\
\hline 11 & $\begin{aligned} & x^{5}+z y^{2 n} \\
n= & 3 ; 4,5 ; 6-10\end{aligned}$ & $\begin{array}{c}t^{2}+z^{3} x+x^{5}+z y^{2} \subset \mathbb{P}(15,8,6,11) \\
\text { Diff }=\left(0,0,0, \frac{n-1}{n}\right)\end{array}$ & $3,5,11$ \\
\hline 12 & $\begin{array}{l}x^{5}+z y^{n}, n=5 ; 7 \\
9 ; 13 ; 15 ; 17,19,21\end{array}$ & $\begin{array}{l}t+z^{3} x+x^{5}+z y \subset \mathbb{P}(15,4,3,11) \\
\mathbb{P}(4,3,11), \text { Diff }=\left(\frac{1}{2}, 0,0, \frac{n-1}{n}\right)\end{array}$ & $\begin{array}{c}6,7,10 \\
14,16,22\end{array}$ \\
\hline 13 & $\begin{array}{c}x^{5}+z^{2} y^{2 n} \\
n=2,3 ; 4,5,6\end{array}$ & $\begin{array}{c}t^{2}+z^{3} x+x^{5}+z^{2} y^{2} \subset \mathbb{P}(15,8,6,7) \\
\text { Diff }=\left(0,0,0, \frac{n-1}{n}\right)\end{array}$ & 6,7 \\
\hline 14 & $\begin{array}{c}x^{5}+z^{2} y^{n} \\
n=5 ; 9,11,13\end{array}$ & $\begin{array}{c}t+z^{3} x+x^{5}+z^{2} y \subset \mathbb{P}(15,4,3,7) \\
\mathbb{P}(4,3,7), \text { Diff }=\left(\frac{1}{2}, 0,0, \frac{n-1}{n}\right)\end{array}$ & 6,14 \\
\hline 15 & $\begin{array}{c}x^{i}\left(x+y^{n}\right)^{j} f_{6-i-j}\left(x, y^{n}\right) \\
n=1 \& i=0 \& j \leqslant 1, \\
n=2 \& i \leqslant 2 \& j \leqslant 4\end{array}$ & $\begin{array}{c}t^{2}+z x+g(x, y) \subset \mathbb{P}(3,5,1,1) \\
\operatorname{Diff}=\left(0, \frac{2}{3}, 0, \frac{n-1}{n}\right)\end{array}$ & 3 \\
\hline 16 & $\begin{array}{c}x^{6}+y^{n} \\
n=7 ; 11 ; 13 ; 17\end{array}$ & $\begin{array}{c}t^{2}+z x+x^{6}+y \subset \mathbb{P}(3,5,1,6) \\
\mathbb{P}(3,5,1), \text { Diff }=\left(0, \frac{2}{3}, 0, \frac{n-1}{n}\right)\end{array}$ & $\begin{array}{c}7,12,13 \\
18\end{array}$ \\
\hline 17 & $\begin{array}{c}x^{3 i} f_{2-i}\left(x^{3}, y^{n}\right) \\
(n=4 ; 5) \& i=0 ;(7,8) \& i \leqslant 1\end{array}$ & $\begin{array}{c}t^{2}+z x+g\left(x, y^{1 / n}\right) \subset \mathbb{P}(3,5,1,3) \\
\text { Diff }=\left(0, \frac{2}{3}, 0, \frac{n-1}{n}\right)\end{array}$ & $4,6,9$ \\
\hline
\end{tabular}


Продолжение таблицы 3

\begin{tabular}{|c|c|c|c|}
\hline 18 & $\begin{array}{c}a z^{2} y x^{2 i} f_{1-i}\left(x^{2}, y^{3}\right)+ \\
+b z y^{2} x^{2 j+1} f_{1-j}\left(x^{2}, y^{3}\right)+ \\
+x^{2 l} f_{3-l}\left(x^{2}, y^{3}\right), \\
i=0 \| j=0|| l=0\end{array}$ & $\begin{array}{c}t^{2}+z^{3} x+g(z, x, y) \subset \mathbb{P}(9,5,3,2) \\
\text { Diff }=\varnothing\end{array}$ & 2 \\
\hline 19 & $\begin{array}{c}x^{2 i} f_{3-i}\left(x^{2}, y^{5}\right) \\
i \leqslant 1\end{array}$ & $\begin{array}{c}t^{2}+z x+g\left(x, y^{1 / 5}\right) \subset \mathbb{P}(3,5,1,2) \\
\operatorname{Diff}=\left(0, \frac{2}{3}, 0, \frac{4}{5}\right)\end{array}$ & 6 \\
\hline 20 & $\begin{array}{c}x^{6}+x^{2} y^{n} \\
n=7 ; 11\end{array}$ & $\begin{array}{c}t^{2}+z x+x^{6}+x^{2} y \subset \mathbb{P}(3,5,1,4) \\
\operatorname{Diff}=\left(0, \frac{2}{3}, 0, \frac{n-1}{n}\right)\end{array}$ & 7,12 \\
\hline 21 & $\begin{array}{c}a z^{2} y^{6}+b z x^{3} y^{3}+x^{6}+ \\
+c x^{2} y^{9},|a|+|c| \neq 0\end{array}$ & $\begin{array}{c}t^{2}+z^{3} x+g\left(z, x, y^{1 / 3}\right) \subset \mathbb{P}(9,5,3,4) \\
\text { Diff }=\left(0,0,0, \frac{2}{3}\right)\end{array}$ & 4 \\
\hline 22 & $\begin{array}{c}x^{6}+z y^{n}, n=5 \\
6,7 ; 8 ; 9,10 ; 11,12\end{array}$ & $\begin{array}{c}t^{2}+z^{3} x+x^{6}+z y \subset \mathbb{P}(9,5,3,13) \\
\text { Diff }=\left(0,0,0, \frac{n-1}{n}\right)\end{array}$ & $\begin{array}{l}5,7,8 \\
10,13\end{array}$ \\
\hline 23 & $\begin{array}{c}x^{6}+z x^{2} y^{n} \\
n=5,6\end{array}$ & $\begin{array}{c}t^{2}+z^{3} x+x^{6}+z x^{2} y \subset \mathbb{P}(9,5,3,7) \\
\text { Diff }=\left(0,0,0, \frac{n-1}{n}\right)\end{array}$ & 7 \\
\hline 24 & $\begin{array}{c}x^{6}+z^{2} y^{n} \\
n=5 ; 7\end{array}$ & $\begin{array}{c}t^{2}+z^{3} x+x^{6}+z^{2} y \subset \mathbb{P}(9,5,3,8) \\
\text { Diff }=\left(0,0,0, \frac{n-1}{n}\right)\end{array}$ & 5,8 \\
\hline 25 & $\begin{array}{c}x^{5} y+y^{n} \\
n=7 ; 9 ; 13 ; 15 ; 19 ; 25\end{array}$ & $\begin{array}{c}t+z x+x^{5} y+y^{n} \subset \mathbb{P}(5 n, 4 n+1, n-1,5) \\
\mathbb{P}(4 n+1, n-1,5), \text { Diff }=\left(\frac{1}{2}, \frac{2}{3}, 0,0\right)\end{array}$ & $\begin{array}{c}8,10,15 \\
18,22,30\end{array}$ \\
\hline 26 & $\begin{array}{l}x^{5} y+y^{2 n} \\
n=5,6 ; 9,11,12\end{array}$ & $\begin{array}{c}t^{2}+z x+x^{5} y+y^{2 n} \subset \mathbb{P}(5 n, 8 n+1,2 n-1,5) \\
\text { Diff }=\left(0, \frac{2}{3}, 0,0\right)\end{array}$ & 7,15 \\
\hline 27 & $\begin{array}{c}a z x^{2} y^{2 n+1}+x^{5} y+b y^{6 n+2} \\
n=1 \quad b \neq 0 ; 2,3\end{array}$ & $\begin{array}{c}t^{2}+z^{3} x+g(z, x, y) \subset \mathbb{P}(15 n+5,8 n+3,6 n+1,5) \\
\text { Diff }=\varnothing\end{array}$ & 4,5 \\
\hline 28 & $\begin{array}{c}a z x^{2} y^{n}+x^{5} y+b y^{3 n-1} \\
n=6,8\end{array}$ & $\begin{array}{c}t+z^{3} x+g(z, x, y) \subset \mathbb{P}(15 n-5,4 n-1,3 n-2,5) \\
\mathbb{P}(4 n-1,3 n-2,5), \text { Diff }=\left(\frac{1}{2}, 0,0,0\right)\end{array}$ & 10 \\
\hline $\begin{array}{l}29 \\
(2)\end{array}$ & $\begin{array}{l}z f_{4}\left(x, y^{2}\right)+z^{2} y^{5}+ \\
\quad+y f_{5}\left(x, y^{2}\right)\end{array}$ & $\begin{array}{c}t+z^{3} x+g(z, x, y) \subset \mathbb{P}(11,3,2,1) \\
\mathbb{P}(3,2,1), \operatorname{Diff}=\left(\frac{1}{2}, 0,0,0\right)\end{array}$ & 2 \\
\hline 30 & $\begin{array}{c}y x^{i} f_{5-i}\left(x, y^{3}\right) \\
i \leqslant 3\end{array}$ & $\begin{array}{c}t^{2}+z x+g(x, y) \subset \mathbb{P}(8,13,3,1) \\
\text { Diff }=\left(0, \frac{2}{3}, 0,0\right)\end{array}$ & 3 \\
\hline 31 & $\begin{array}{c}y x^{i} f_{5-i}\left(x, y^{4}\right) \\
i \leqslant 3\end{array}$ & $\begin{array}{c}t+z x+g(x, y) \subset \mathbb{P}(21,17,4,1) \\
\mathbb{P}(17,4,1), \text { Diff }=\left(\frac{1}{2}, \frac{2}{3}, 0,0\right)\end{array}$ & 6 \\
\hline 32 & $\begin{array}{l}x^{5} y+x^{2} y^{n} \\
n=9 ; 11 ; 15\end{array}$ & $\begin{array}{c}t+z x+x^{5} y+x^{2} y^{n} \subset \mathbb{P}(5 n-2,4 n-1, n-1,3) \\
\mathbb{P}(4 n-1, n-1,3), \text { Diff }=\left(\frac{1}{2}, \frac{2}{3}, 0,0\right)\end{array}$ & $10,12,18$ \\
\hline 33 & $\begin{array}{c}x^{5} y+x^{2} y^{2 n} \\
n=4,6,7\end{array}$ & $\begin{array}{c}t^{2}+z x+x^{5} y+x^{2} y^{2 n} \subset \mathbb{P}(5 n-1,8 n-1,2 n-1,3) \\
\text { Diff }=\left(0, \frac{2}{3}, 0,0\right)\end{array}$ & 9 \\
\hline 34 & $\begin{array}{l}x^{5} y+z y^{2 n+1} \\
n=2 ; 3,4 ; 5-8\end{array}$ & $\begin{array}{c}t^{2}+z^{3} x+g(z, x, y) \subset \mathbb{P}(15 n+8,8 n+5,6 n+1,11) \\
\text { Diff }=\varnothing\end{array}$ & $3,5,11$ \\
\hline 35 & $\begin{array}{c}x^{5} y+z y^{n}, n=6 \\
10,12 ; 14 ; 16,18\end{array}$ & $\begin{array}{c}t+z^{3} x+x^{5} y+z y^{n} \subset \mathbb{P}(15 n+1,4 n+1,3 n-2,11) \\
\mathbb{P}(4 n+1,3 n-2,11), \text { Diff }=\left(\frac{1}{2}, 0,0,0\right)\end{array}$ & $\begin{array}{c}7,14,16 \\
22\end{array}$ \\
\hline
\end{tabular}




\begin{tabular}{|c|c|c|c|}
\hline 36 & $\begin{array}{l}x^{5} y+z^{2} y^{n} \\
n=3 ; 7 ; 9,11\end{array}$ & $\begin{array}{c}t+z^{3} x+g(z, x, y) \subset \mathbb{P}(15 n+2,4 n+1,3 n-1,7) \\
\mathbb{P}(4 n+1,3 n-1,7), \text { Diff }=\left(\frac{1}{2}, 0,0,0\right)\end{array}$ & $6,8,14$ \\
\hline 37 & $\begin{array}{l}x^{5} y+z^{2} y^{2 n} \\
n=2 ; 3,4,5\end{array}$ & $\begin{aligned} & t^{2}+z^{3} x+g(z, x, y) \subset \mathbb{P}(15 n+1,8 n+1,6 n-1,7) \\
& \text { Diff }=\varnothing\end{aligned}$ & 6,7 \\
\hline $\begin{array}{l}38 \\
(3)\end{array}$ & $z f_{5}(x, y)+f_{7}(x, y)$ & $\begin{array}{c}t+z^{3} x+g(z, x, y) \subset \mathbb{P}(7,2,1,1) \\
\mathbb{P}(2,1,1), \text { Diff }=\left(\frac{1}{2}, 0,0,0\right)\end{array}$ & 2 \\
\hline 39 & $\begin{array}{c}x^{3} z^{i} f_{2-i}\left(z, x^{2}\right)+y^{2 n} \\
n=4,5 ; 6\end{array}$ & $\begin{array}{c}t^{2}+z^{3} x+g\left(z, x, y^{1 / n}\right) \subset \mathbb{P}(7,4,2,7) \\
\text { Diff }=\left(0,0,0, \frac{n-1}{n}\right)\end{array}$ & 5,7 \\
\hline 40 & $\begin{array}{c}x^{3} z^{i} f_{2-i}\left(z, x^{2}\right)+y^{n} \\
n=9 ; 11 ; 13\end{array}$ & $\begin{array}{c}t+z^{3} x+g\left(z, x, y^{1 / n}\right) \subset \mathbb{P}(7,2,1,7) \\
\mathbb{P}(2,1,7), \text { Diff }=\left(\frac{1}{2}, 0,0, \frac{n-1}{n}\right)\end{array}$ & $10,12,14$ \\
\hline 41 & $\begin{array}{c}x^{3} z^{i} f_{2-i}\left(z, x^{2}\right)+a z^{2} y^{4}+ \\
+b z x^{2} y^{4}+c x^{4} y^{4}+d x y^{8} \\
|a|+|d| \neq 0\end{array}$ & $\begin{array}{c}t^{2}+z^{3} x+g\left(z, x, y^{1 / 2}\right) \subset \mathbb{P}(7,4,2,3) \\
\operatorname{Diff}=\left(0,0,0, \frac{1}{2}\right)\end{array}$ & 3 \\
\hline 42 & $\begin{array}{c}x^{3} z^{i} f_{2-i}\left(z, x^{2}\right)+a z^{2} y^{5}+ \\
+b z x^{2} y^{5}+c x^{4} y^{5}+d x y^{10} \\
|a|+|b|+|d| \neq 0\end{array}$ & $\begin{array}{c}t+z^{3} x+g\left(z, x, y^{1 / 5}\right) \subset \mathbb{P}(7,2,1,3) \\
\mathbb{P}(2,1,3), \text { Diff }=\left(\frac{1}{2}, 0,0, \frac{4}{5}\right)\end{array}$ & 6 \\
\hline 43 & $\begin{array}{l}x^{3} z^{i} f_{2-i}\left(z, x^{2}\right)+a z y^{2 n}+ \\
\quad+b x^{2} y^{2 n}, n=3 a \neq 0 ; 4\end{array}$ & $\begin{array}{c}t^{2}+z^{3} x+g\left(z, x, y^{1 / n}\right) \subset \mathbb{P}(7,4,2,5) \\
\text { Diff }=\left(0,0,0, \frac{n-1}{n}\right)\end{array}$ & 3,5 \\
\hline 44 & $\begin{array}{l}x^{3} z^{i} f_{2-i}\left(z, x^{2}\right)+a z y^{n}+ \\
\quad+b x^{2} y^{n}, n=7 ; 9\end{array}$ & $\begin{array}{l}t+z^{3} x+g\left(z, x, y^{1 / n}\right) \subset \mathbb{P}(7,2,1,5) \\
\mathbb{P}(2,1,5), \text { Diff }=\left(\frac{1}{2}, 0,0, \frac{n-1}{n}\right)\end{array}$ & 8,10 \\
\hline 45 & $\begin{array}{c}x^{6} y+y^{n} \\
n=8,12,14\end{array}$ & $\begin{array}{c}t^{2}+z x+x^{6} y+y^{n} \subset \mathbb{P}(3 n, 5 n+1, n-1,6) \\
\text { Diff }=\left(0, \frac{2}{3}, 0,0\right)\end{array}$ & 9 \\
\hline 46 & $\begin{array}{l}x^{6} y+b x^{3} y^{n+1}+c y^{2 n+1} \\
(c \neq 0 \&(n=4,5)) ; 7\end{array}$ & $\begin{array}{c}t+z x+g(x, y) \subset \mathbb{P}(6 n+3,5 n+3, n, 3) \\
\text { Diff }=\left(\frac{1}{2}, \frac{2}{3}, 0,0\right)\end{array}$ & 6,18 \\
\hline 47 & $\begin{array}{c}y x^{2 i} f_{3-i}\left(x^{2}, y^{3}\right) \\
i \leqslant 1\end{array}$ & $\begin{array}{c}t^{2}+z x+g(x, y) \subset \mathbb{P}(10,17,3,2) \\
\text { Diff }=\left(0, \frac{2}{3}, 0,0\right)\end{array}$ & 3 \\
\hline 48 & $\begin{array}{c}y x^{i}\left(x+y^{2}\right)^{j} f_{6-i-j}\left(x, y^{2}\right) \\
i \leqslant 3 \& j \leqslant 5\end{array}$ & $\begin{array}{c}t+z x+g(x, y) \subset \mathbb{P}(13,11,2,1) \\
\text { Diff }=\left(\frac{1}{2}, \frac{2}{3}, 0,0\right)\end{array}$ & 6 \\
\hline 49 & $x^{6} y+x^{2} y^{10}$ & $\begin{array}{c}t^{2}+z x+x^{6} y+x^{2} y^{10} \subset \mathbb{P}(29,49,9,4) \\
\text { Diff }=\left(0, \frac{2}{3}, 0,0\right)\end{array}$ & 12 \\
\hline 50 & $\begin{array}{l}a z^{2} y^{5}+b z x^{3} y^{3}+x^{6} y+ \\
\quad+c x^{2} y^{8},|a|+|c| \neq 0\end{array}$ & $\begin{array}{c}t^{2}+z^{3} x+g(z, x, y) \subset \mathbb{P}(23,13,7,4) \\
\text { Diff }=\varnothing\end{array}$ & 4 \\
\hline 51 & $\begin{aligned} & x^{6} y+z y^{n} \\
n= & 6,7,8,9 ; 10,11\end{aligned}$ & $\begin{array}{c}t+z^{3} x+x^{6} y+z y^{n} \subset \mathbb{P}(18 n+1,5 n+1,3 n-2,13) \\
\mathbb{P}(5 n+1,3 n-2,13), \text { Diff }=\left(\frac{1}{2}, 0,0,0\right)\end{array}$ & 10,26 \\
\hline 52 & $\begin{array}{c}x^{6} y+z x^{2} y^{n} \\
n=5,6\end{array}$ & $\begin{array}{c}t+z^{3} x+x^{6} y+z x^{2} y^{n} \subset \mathbb{P}(18 n-5,5 n-1,3 n-2,7) \\
\mathbb{P}(5 n-1,3 n-2,7), \text { Diff }=\left(\frac{1}{2}, 0,0,0\right)\end{array}$ & 14 \\
\hline 53 & $\begin{array}{c}x^{5} y^{2}+y^{4 n} \\
n=2 ; 5\end{array}$ & $\begin{array}{c}t^{2}+z x+x^{5} y+y^{2 n} \subset \mathbb{P}(5 n, 8 n+1,2 n-1,5) \\
\text { Diff }=\left(0, \frac{2}{3}, 0, \frac{1}{2}\right)\end{array}$ & 7,15 \\
\hline
\end{tabular}


Продолжение таблицы 3

\begin{tabular}{|c|c|c|c|}
\hline 54 & $\begin{array}{c}x^{5} y^{2}+y^{2 n} \\
n=7 ; 9\end{array}$ & $\begin{array}{c}t^{2}+z x+g(x, y) \subset \mathbb{P}(5 n, 8 n+2,2 n-2,5) \\
\text { Diff }=\left(0, \frac{2}{3}, 0,0\right)\end{array}$ & 9,15 \\
\hline 55 & $\begin{aligned} & x^{5} y^{2}+y^{n} \\
& n=9 ; 11 ; 15 ; 21\end{aligned}$ & $\begin{array}{c}t+z x+g(x, y) \subset \mathbb{P}(5 n, 4 n+2, n-2,5) \\
\mathbb{P}(4 n+2, n-2,5) \text { Diff }=\left(\frac{1}{2}, \frac{2}{3}, 0,0\right)\end{array}$ & $\begin{array}{c}12,15,22 \\
30\end{array}$ \\
\hline 56 & $\begin{array}{c}a z x^{2} y^{4}+x^{5} y^{2}+b y^{10} \\
b \neq 0\end{array}$ & $\begin{array}{c}t^{2}+z^{3} x+g(z, x, y) \subset \mathbb{P}(25,14,8,5) \\
\text { Diff }=\varnothing\end{array}$ & 4 \\
\hline 57 & $\begin{array}{c}a z x^{2} y^{n}+x^{5} y^{2}+b y^{3 n-2} \\
n=5,7\end{array}$ & $\begin{array}{c}t+z^{3} x+g(z, x, y) \subset \mathbb{P}(15 n-10,4 n-2,3 n-4,5) \\
\mathbb{P}(4 n-2,3 n-4,5), \text { Diff }=\left(\frac{1}{2}, 0,0,0\right)\end{array}$ & 10 \\
\hline 58 & $a z x^{2} y^{6}+x^{5} y^{2}+b y^{16}$ & $\begin{array}{c}t^{2}+z^{3} x+g\left(z, x, y^{1 / 2}\right) \subset \mathbb{P}(20,11,7,5) \\
\text { Diff }=\left(0,0,0, \frac{1}{2}\right)\end{array}$ & 5 \\
\hline 59 & $\begin{array}{l}y^{2} x^{i} f_{5-i}\left(x, y^{3}\right) \\
i \leqslant 3\end{array}$ & $\begin{array}{c}t+z x+g(x, y) \subset \mathbb{P}(17,14,3,1) \\
\mathbb{P}(14,3,1), \text { Diff }=\left(\frac{1}{2}, \frac{2}{3}, 0,0\right)\end{array}$ & 6 \\
\hline 60 & $x^{5} y^{2}+x^{2} y^{10}$ & $\begin{array}{c}t^{2}+z x+x^{5} y^{2}+x^{2} y^{10} \subset \mathbb{P}(23,38,8,3) \\
\text { Diff }=\left(0, \frac{2}{3}, 0,0\right)\end{array}$ & 9 \\
\hline 61 & $\begin{array}{c}x^{5} y^{2}+x^{2} y^{n} \\
n=7 ; 9 ; 13\end{array}$ & $\begin{array}{c}t+z x+x^{5} y^{2}+x^{2} y^{n} \subset \mathbb{P}(5 n-4,4 n-2, n-2,3) \\
\mathbb{P}(4 n-2, n-2,3), \text { Diff }=\left(\frac{1}{2}, \frac{2}{3}, 0,0\right)\end{array}$ & $10,12,18$ \\
\hline 62 & $x^{5} y^{2}+x^{2} y^{12}$ & $\begin{array}{c}t^{2}+z x+x^{5} y+x^{2} y^{6} \subset \mathbb{P}(14,23,5,3) \\
\text { Diff }=\left(0, \frac{2}{3}, 0, \frac{1}{2}\right)\end{array}$ & 9 \\
\hline 63 & $\begin{array}{c}x^{5} y^{2}+z y^{4 n+2} \\
n=1 ; 2,3\end{array}$ & $\begin{array}{c}t^{2}+z^{3} x+g\left(z, x, y^{1 / 2}\right) \subset \mathbb{P}(15 n+8,8 n+5,6 n+1,11) \\
\text { Diff }=\left(0,0,0, \frac{1}{2}\right)\end{array}$ & 5,11 \\
\hline 64 & $\begin{array}{c}\quad x^{5} y^{2}+z y^{n} \\
n=7 ; 9,11 ; 13,15\end{array}$ & $\begin{array}{c}t+z^{3} x+x^{5} y^{2}+z y^{n} \subset \mathbb{P}(15 n+2,4 n+2,3 n-4,11) \\
\mathbb{P}(4 n+2,3 n-4,11), \text { Diff }=\left(\frac{1}{2}, 0,0,0\right)\end{array}$ & $10,16,22$ \\
\hline 65 & $\begin{array}{c}x^{5} y^{2}+z y^{4 n} \\
n=2 ; 3\end{array}$ & $\begin{array}{c}t^{2}+z^{3} x+g(z, x, y) \subset \mathbb{P}(30 n+1,16 n+2,12 n-4,11) \\
\text { Diff }=\varnothing\end{array}$ & 5,11 \\
\hline 66 & $x^{5} y^{2}+z^{2} y^{6}$ & $\begin{array}{c}t^{2}+z^{3} x+g(z, x, y) \subset \mathbb{P}(47,26,16,7) \\
\text { Diff }=\varnothing\end{array}$ & 7 \\
\hline 67 & $\begin{array}{c}x^{5} y^{2}+z^{2} y^{4 n} \\
n=1 ; 2\end{array}$ & $\begin{array}{c}t^{2}+z^{3} x+g\left(z, x, y^{1 / 2}\right) \subset \mathbb{P}(15 n+1,8 n+1,6 n-1,7) \\
\text { Diff }=\left(0,0,0, \frac{1}{2}\right)\end{array}$ & 6,7 \\
\hline 68 & $\begin{array}{c}x^{5} y^{2}+z^{2} y^{n} \\
n=5 ; 7,9\end{array}$ & $\begin{array}{c}t+z^{3} x+x^{5} y^{2}+z^{2} y^{n} \subset \mathbb{P}(15 n+4,4 n+2,3 n-2,7) \\
\mathbb{P}(4 n+2,3 n-2,7), \text { Diff }=\left(\frac{1}{2}, 0,0,0\right)\end{array}$ & 8,14 \\
\hline 69 & $\begin{array}{c}x^{i}(x+y)^{j} f_{8-i-j}(x, y) \\
i \leqslant 1 \& j \leqslant 3\end{array}$ & $\begin{array}{c}t^{2}+z x+g(x, y) \subset \mathbb{P}(4,7,1,1) \\
\operatorname{Diff}=\left(0, \frac{2}{3}, 0,0\right)\end{array}$ & 3 \\
\hline 70 & $x^{8}+a z x^{3} y^{3}+y^{9}$ & $\begin{array}{c}t^{2}+z^{3} x+x^{8}+a z x^{3} y+y^{3} \subset \mathbb{P}(12,7,3,8) \\
\text { Diff }=\left(0,0,0, \frac{2}{3}\right)\end{array}$ & 4 \\
\hline 71 & $x^{8}+a x^{4} y^{5}+y^{10}$ & $\begin{array}{c}t^{2}+z x+x^{8}+a x^{4} y+y^{2} \subset \mathbb{P}(4,7,1,4) \\
\text { Diff }=\left(0, \frac{2}{3}, 0, \frac{4}{5}\right)\end{array}$ & 6 \\
\hline 72 & $x^{8}+y^{11}$ & $\begin{array}{l}t^{2}+z x+x^{8}+y \subset \mathbb{P}(4,7,1,8) \\
\mathbb{P}(4,7,1), \text { Diff }=\left(0, \frac{2}{3}, 0, \frac{10}{11}\right)\end{array}$ & 12 \\
\hline
\end{tabular}


Продолжение таблицы 3

\begin{tabular}{|c|c|c|c|}
\hline 73 & $x^{8}+x^{2} y^{7}$ & $\begin{array}{c}t^{2}+z x+x^{8}+x^{2} y \subset \mathbb{P}(4,7,1,6) \\
\text { Diff }=\left(0, \frac{2}{3}, 0, \frac{6}{7}\right)\end{array}$ & 9 \\
\hline 74 & $x^{8}+a x^{5} y^{4}+x^{2} y^{8}$ & $\begin{array}{c}t^{2}+z x+x^{8}+a x^{5} y+x^{2} y^{2} \subset \mathbb{P}(4,7,1,3) \\
\text { Diff }=\left(0, \frac{2}{3}, 0, \frac{3}{4}\right)\end{array}$ & 9 \\
\hline 75 & $\begin{array}{c}c z^{2} y^{4}+\underline{a} z x^{4} y^{2}+\underline{b} x^{8}+ \\
+d x^{3} y^{6}, c \neq 0\end{array}$ & $\begin{array}{c}t^{2}+z^{3} x+g\left(z, x, y^{1 / 2}\right) \subset \mathbb{P}(12,7,3,5) \\
\text { Diff }=\left(0,0,0, \frac{1}{2}\right)\end{array}$ & 5 \\
\hline 76 & $x^{8}+x^{3} y^{7}$ & $\begin{array}{c}t^{2}+z x+x^{8}+x^{3} y \subset \mathbb{P}(4,7,1,5) \\
\text { Diff }=\left(0, \frac{2}{3}, 0, \frac{6}{7}\right)\end{array}$ & 15 \\
\hline 77 & $\begin{array}{l}x^{8}+z y^{n} \\
n=6,7 ; 8\end{array}$ & $\begin{array}{c}t^{2}+z^{3} x+x^{8}+z y \subset \mathbb{P}(12,7,3,17) \\
\text { Diff }=\left(0,0,0, \frac{n-1}{n}\right)\end{array}$ & 7,17 \\
\hline 78 & $x^{8}+z x^{2} y^{5}$ & $\begin{array}{c}t^{2}+z^{3} x+x^{8}+z x^{2} y \subset \mathbb{P}(12,7,3,11) \\
\text { Diff }=\left(0,0,0, \frac{4}{5}\right)\end{array}$ & 11 \\
\hline 79 & $\begin{array}{l}x^{7} y+y^{n} \\
n=9 ; 11\end{array}$ & $\begin{array}{c}t+z x+x^{7} y+y^{n} \subset \mathbb{P}(7 n, 6 n+1, n-1,7) \\
\mathbb{P}(6 n+1, n-1,7), \text { Diff }=\left(\frac{1}{2}, \frac{2}{3}, 0,0\right)\end{array}$ & 10,12 \\
\hline 80 & $\begin{array}{c}x^{7} y+y^{2 n} \\
n=5 ; 6\end{array}$ & $\begin{array}{c}t^{2}+z x+x^{7} y+y^{2 n} \subset \mathbb{P}(7 n, 12 n+1,2 n-1,7) \\
\text { Diff }=\left(0, \frac{2}{3}, 0,0\right)\end{array}$ & 12,21 \\
\hline 81 & $\begin{array}{c}x^{7} y+x^{2} y^{n} \\
n=7 ; 9\end{array}$ & $\begin{array}{c}t+z x+g(x, y) \subset \mathbb{P}(7 n-2,6 n-1, n-1,5) \\
\mathbb{P}(6 n-1, n-1,5), \text { Diff }=\left(\frac{1}{2}, \frac{2}{3}, 0,0\right)\end{array}$ & 16,30 \\
\hline 82 & $x^{7} y+x^{2} y^{8}$ & $\begin{array}{c}t^{2}+z x+g(x, y) \subset \mathbb{P}(27,47,7,5) \\
\operatorname{Diff}=\left(0, \frac{2}{3}, 0,0\right)\end{array}$ & 15 \\
\hline 83 & $\begin{array}{c}x^{7} y+z y^{n} \\
n=6 ; 8\end{array}$ & $\begin{array}{c}t+z^{3} x+g(z, x, y) \subset \mathbb{P}(21 n+1,6 n+1,3 n-2,15) \\
\mathbb{P}(6 n+1,3 n-2,15), \text { Diff }=\left(\frac{1}{2}, 0,0,0\right)\end{array}$ & 10 \\
\hline 84 & $x^{7} y+z y^{7}$ & $\begin{array}{c}t^{2}+z^{3} x+g(z, x, y) \subset \mathbb{P}(74,43,19,15) \\
\text { Diff }=\varnothing\end{array}$ & 5 \\
\hline 85 & $x^{7} y+z x^{2} y^{5}$ & $\begin{array}{c}t^{2}+z^{3} x+g(z, x, y) \subset \mathbb{P}(50,29,13,9) \\
\text { Diff }=\varnothing\end{array}$ & 9 \\
\hline 86 & $x^{7} y+z^{2} y^{4}$ & $\begin{array}{c}t^{2}+z^{3} x+g(z, x, y) \subset \mathbb{P}(43,25,11,9) \\
\text { Diff }=\varnothing\end{array}$ & 9 \\
\hline 87 & $x^{7} y+z^{2} y^{5}$ & $\begin{array}{c}t+z^{3} x+g(z, x, y) \subset \mathbb{P}(107,31,14,9) \\
\mathbb{P}(31,14,9), \text { Diff }=\left(\frac{1}{2}, 0,0,0\right)\end{array}$ & 18 \\
\hline 88 & $\begin{array}{c}x^{6} y^{2}+y^{n} \\
n=9 ; 13\end{array}$ & $\begin{array}{c}t^{2}+z x+x^{6} y^{2}+y^{n} \subset \mathbb{P}(3 n, 5 n+2, n-2,6) \\
\text { Diff }=\left(0, \frac{2}{3}, 0,0\right)\end{array}$ & 12,18 \\
\hline 89 & $\begin{array}{c}x^{6} y^{2}+a x^{3} y^{n}+b y^{2 n-2} \\
n=6 \quad b \neq 0 ; 7\end{array}$ & $\begin{array}{c}t^{2}+z x+g(x, y) \subset \mathbb{P}(3 n-3,5 n-4, n-2,3) \\
\text { Diff }=\left(0, \frac{2}{3}, 0,0\right)\end{array}$ & 6,9 \\
\hline 90 & $\begin{array}{c}y^{2} x^{2 i} f_{3-i}\left(x^{2}, y^{3}\right) \\
i \leqslant 1\end{array}$ & $\begin{array}{c}t^{2}+z x+g(x, y) \subset \mathbb{P}(11,19,3,2) \\
\text { Diff }=\left(0, \frac{2}{3}, 0,0\right)\end{array}$ & 6 \\
\hline 91 & $\begin{array}{l}x^{6} y^{2}+a z^{2} y^{4}+b z x^{3} y^{3}+ \\
+c x^{2} y^{7},|a|+|c| \neq 0\end{array}$ & $\begin{array}{c}t^{2}+z^{3} x+g(z, x, y) \subset \mathbb{P}(19,11,5,4) \\
\text { Diff }=\varnothing\end{array}$ & 4 \\
\hline
\end{tabular}


Продолжение таблицы 3

\begin{tabular}{|c|c|c|c|}
\hline 92 & $x^{6} y^{2}+x^{2} y^{9}$ & $\begin{array}{c}t^{2}+z x+g(x, y) \subset \mathbb{P}(25,43,7,4) \\
\operatorname{Diff}=\left(0, \frac{2}{3}, 0,0\right)\end{array}$ & 12 \\
\hline 93 & $\begin{array}{l}x^{6} y^{2}+z y^{n} \\
n=6 ; 7,8 ; 9\end{array}$ & $\begin{aligned} t^{2}+z^{3} x+g(z, x, y) & \subset \mathbb{P}(9 n+1,5 n+2,3 n-4,13) \\
& \text { Diff }=\varnothing\end{aligned}$ & $7,10,13$ \\
\hline 94 & $x^{6} y^{2}+z x^{2} y^{5}$ & $\begin{array}{c}t^{2}+z^{3} x+g(z, x, y) \subset \mathbb{P}(40,23,11,7) \\
\text { Diff }=\varnothing\end{array}$ & 7 \\
\hline 95 & $x^{6} y^{2}+z^{2} y^{5}$ & $\begin{array}{c}t^{2}+z^{3} x+g(z, x, y) \subset \mathbb{P}(47,27,13,8) \\
\text { Diff }=\varnothing\end{array}$ & 8 \\
\hline 96 & $\begin{array}{c}a z x^{2} y^{4}+x^{5} y^{3}+b y^{9} \\
b \neq 0\end{array}$ & $\begin{array}{c}t+z^{3} x+g(z, x, y) \subset \mathbb{P}(45,13,6,5) \\
\mathbb{P}(13,6,5), \text { Diff }=\left(\frac{1}{2}, 0,0,0\right)\end{array}$ & 4 \\
\hline 97 & $a z x^{2} y^{5}+x^{5} y^{3}+b y^{12}$ & $\begin{array}{c}t^{2}+z^{3} x+g(z, x, y) \subset \mathbb{P}(30,17,9,5) \\
\text { Diff }=\varnothing\end{array}$ & 5 \\
\hline 98 & $a z x^{2} y^{6}+x^{5} y^{3}+b y^{15}$ & $\begin{array}{c}t+z^{3} x+g\left(z, x, y^{1 / 3}\right) \subset \mathbb{P}(25,7,4,5) \\
\mathbb{P}(7,4,5), \text { Diff }=\left(\frac{1}{2}, 0,0, \frac{2}{3}\right)\end{array}$ & 10 \\
\hline 99 & $\begin{array}{c}x^{5} y^{3}+y^{2 n} \\
n=5 ; 7,8\end{array}$ & $\begin{array}{c}t^{2}+z x+g(x, y) \subset \mathbb{P}(5 n, 8 n+3,2 n-3,5) \\
\text { Diff }=\left(0, \frac{2}{3}, 0,0\right)\end{array}$ & 9,15 \\
\hline 100 & $\begin{array}{c}x^{5} y^{3}+y^{n} \\
n=11 ; 17 \\
\end{array}$ & $\begin{array}{c}t+z x+g(x, y) \subset \mathbb{P}(5 n, 4 n+3, n-3,5) \\
\mathbb{P}(4 n+3, n-3,5), \text { Diff }=\left(\frac{1}{2}, \frac{2}{3}, 0,0\right)\end{array}$ & 22,30 \\
\hline 101 & $\begin{array}{c}y x^{i}\left(x+y^{2}\right)^{j} f_{6-i-j}\left(x, y^{2}\right) \\
i \leqslant 3 \& j \leqslant 5\end{array}$ & $\begin{array}{c}t+z x+g(x, y) \subset \mathbb{P}(13,11,2,1) \\
\mathbb{P}(11,2,1), \text { Diff }=\left(\frac{1}{2}, \frac{2}{3}, 0,0\right)\end{array}$ & 6 \\
\hline 102 & $\begin{array}{c}x^{5} y^{3}+x^{2} y^{n} \\
n=7 ; 11\end{array}$ & $\begin{array}{c}t+z x+g(x, y) \subset \mathbb{P}(5 n-6,4 n-3, n-3,3) \\
\mathbb{P}(4 n-3, n-3,3), \text { Diff }=\left(\frac{1}{2}, \frac{2}{3}, 0,0\right)\end{array}$ & 10,18 \\
\hline 103 & $\begin{array}{c}x^{5} y^{3}+x^{2} y^{2 n} \\
n=4,5\end{array}$ & $\begin{array}{c}t^{2}+z x+g(x, y) \subset \mathbb{P}(5 n-3,8 n-3,2 n-3,3) \\
\text { Diff }=\left(0, \frac{2}{3}, 0,0\right)\end{array}$ & 9 \\
\hline 104 & $\begin{array}{c}x^{5} y^{3}+z y^{3 n} \\
n=2 ; 4\end{array}$ & $\begin{array}{c}t+z^{3} x+x^{5} y+z y^{n} \subset \mathbb{P}(15 n+1,4 n+1,3 n-2,11) \\
\mathbb{P}(4 n+1,3 n-2,11), \text { Diff }=\left(\frac{1}{2}, 0,0, \frac{2}{3}\right)\end{array}$ & 10,22 \\
\hline 105 & $\begin{array}{c}x^{5} y^{3}+z y^{2 n+1} \\
n=3 ; 5\end{array}$ & $\begin{array}{c}t^{2}+z^{3} x+g(z, x, y) \subset \mathbb{P}(15 n+9,8 n+7,6 n-3,11) \\
\text { Diff }=\varnothing\end{array}$ & 5,11 \\
\hline 106 & $\begin{array}{c}x^{5} y^{3}+z y^{n} \\
n=8 ; 10\end{array}$ & $\begin{array}{c}t+z^{3} x+g(z, x, y) \subset \mathbb{P}(15 n+3,4 n+3,3 n-6,11) \\
\mathbb{P}(4 n+3,3 n-6,11), \text { Diff }=\left(\frac{1}{2}, 0,0,0\right)\end{array}$ & 16 \\
\hline 107 & $x^{5} y^{3}+z y^{9}$ & $\begin{array}{c}t^{2}+z^{3} x+x^{5} y+z y^{3} \subset \mathbb{P}(23,13,7,11) \\
\text { Diff }=\left(0,0,0, \frac{2}{3}\right)\end{array}$ & 11 \\
\hline 108 & $x^{5} y^{3}+z^{2} y^{4}$ & $\begin{array}{c}t^{2}+z^{3} x+g(z, x, y) \subset \mathbb{P}(33,19,9,7) \\
\text { Diff }=\varnothing\end{array}$ & 7 \\
\hline 109 & $\begin{array}{c}x^{5} y^{3}+z^{2} y^{n} \\
n=5 ; 7\end{array}$ & $\begin{array}{c}t+z^{3} x+g(z, x, y) \subset \mathbb{P}(15 n+6,4 n+3,3 n-3,7) \\
\mathbb{P}(4 n+3,3 n-3,7), \text { Diff }=\left(\frac{1}{2}, 0,0,0\right)\end{array}$ & 8,14 \\
\hline 110 & $x^{5} y^{3}+z^{2} y^{6}$ & $\begin{array}{c}t^{2}+z^{3} x+x^{5} y+z^{2} y^{2} \subset \mathbb{P}(16,9,5,7) \\
\text { Diff }=\left(0,0,0, \frac{2}{3}\right)\end{array}$ & 7 \\
\hline
\end{tabular}




\begin{tabular}{|c|c|c|c|}
\hline 111 & $\begin{array}{c}x^{i}(x+y)^{j} y^{k} f_{9-i-j-k}(x, y) \\
i \leqslant 2 \& j \leqslant 4 \& k \leqslant 4\end{array}$ & $\begin{array}{l}t+z x+g(x, y) \subset \mathbb{P}(9,8,1,1) \\
\mathbb{P}(8,1,1), \text { Diff }=\left(\frac{1}{2}, \frac{2}{3}, 0,0\right)\end{array}$ & 6 \\
\hline 112 & $x^{9}+y^{10}$ & $\begin{array}{c}t^{2}+z x+x^{9}+y^{2} \subset \mathbb{P}(9,16,2,9) \\
\text { Diff }=\left(0, \frac{2}{3}, 0, \frac{4}{5}\right)\end{array}$ & 15 \\
\hline 113 & $x^{9}+x^{2} y^{8}$ & $\begin{array}{c}t^{2}+z x+x^{9}+x^{2} y^{2} \subset \mathbb{P}(9,16,2,7) \\
\text { Diff }=\left(0, \frac{2}{3}, 0, \frac{3}{4}\right)\end{array}$ & 21 \\
\hline 114 & $x^{9}+z y^{7}$ & $\begin{array}{c}t+z^{3} x+x^{9}+z y \subset \mathbb{P}(27,8,3,19) \\
\mathbb{P}(8,3,19), \text { Diff }=\left(\frac{1}{2}, 0,0, \frac{6}{7}\right)\end{array}$ & 14 \\
\hline 115 & $x^{9}+z x^{2} y^{5}$ & $\begin{array}{c}t+z^{3} x+x^{9}+z x^{2} y \subset \mathbb{P}(27,8,3,13) \\
\mathbb{P}(8,3,13), \text { Diff }=\left(\frac{1}{2}, 0,0, \frac{4}{5}\right)\end{array}$ & 26 \\
\hline 116 & $x^{9}+z^{2} y^{4}$ & $\begin{array}{c}t^{2}+z^{3} x+x^{9}+z^{2} y^{2} \subset \mathbb{P}(27,16,6,11) \\
\text { Diff }=\left(0,0,0, \frac{1}{2}\right)\end{array}$ & 11 \\
\hline 117 & $x^{8} y+y^{10}$ & $\begin{array}{c}t^{2}+z x+g(x, y) \subset \mathbb{P}(40,71,9,8) \\
\operatorname{Diff}=\left(0, \frac{2}{3}, 0,0\right)\end{array}$ & 12 \\
\hline 118 & $x^{8} y+x^{2} y^{8}$ & $\begin{array}{c}t^{2}+z x+x^{8} y+x^{2} y^{8} \subset \mathbb{P}(31,55,7,6) \\
\text { Diff }=\left(0, \frac{2}{3}, 0,0\right)\end{array}$ & 18 \\
\hline 119 & $x^{8} y+x^{3} y^{7}$ & $\begin{array}{c}t+z x+x^{8} y+x^{3} y^{7} \subset \mathbb{P}(53,47,6,5) \\
\mathbb{P}(47,6,5), \text { Diff }=\left(\frac{1}{2}, \frac{2}{3}, 0,0\right)\end{array}$ & 30 \\
\hline 120 & $x^{8} y+z y^{7}$ & $\begin{array}{c}t+z^{3} x+g(z, x, y) \subset \mathbb{P}(169,50,19,17) \\
\mathbb{P}(50,19,17), \text { Diff }=\left(\frac{1}{2}, 0,0,0\right)\end{array}$ & 24 \\
\hline 121 & $x^{8} y+z x^{2} y^{5}$ & $\begin{array}{c}t+z^{3} x+g(z, x, y) \subset \mathbb{P}(115,34,13,11) \\
\mathbb{P}(34,13,11), \text { Diff }=\left(\frac{1}{2}, 0,0,0\right)\end{array}$ & 22 \\
\hline 122 & $x^{8} y+z^{2} y^{4}$ & $\begin{array}{c}t^{2}+z^{3} x+g(z, x, y) \subset \mathbb{P}(49,29,11,10) \\
\text { Diff }=\varnothing\end{array}$ & 10 \\
\hline 123 & $x^{7} y^{2}+y^{10}$ & $\begin{array}{c}t^{2}+z x+g(x, y) \subset \mathbb{P}(35,62,8,7) \\
\text { Diff }=\left(0, \frac{2}{3}, 0,0\right)\end{array}$ & 12 \\
\hline 124 & $x^{7} y^{2}+y^{11}$ & $\begin{array}{c}t+z x+g(x, y) \subset \mathbb{P}(77,68,9,7) \\
\mathbb{P}(68,9,7), \text { Diff }=\left(\frac{1}{2}, \frac{2}{3}, 0,0\right)\end{array}$ & 42 \\
\hline 125 & $x^{7} y^{2}+x^{2} y^{8}$ & $\begin{array}{c}t^{2}+z x+x^{7} y+x^{2} y^{4} \subset \mathbb{P}(13,23,3,5) \\
\text { Diff }=\left(0, \frac{2}{3}, 0, \frac{1}{2}\right)\end{array}$ & 15 \\
\hline 126 & $x^{7} y^{2}+z y^{7}$ & $\begin{array}{c}t+z^{3} x+g(z, x, y) \subset \mathbb{P}(149,44,17,15) \\
\mathbb{P}(44,17,15), \text { Diff }=\left(\frac{1}{2}, 0,0,0\right)\end{array}$ & 10 \\
\hline 127 & $x^{7} y^{2}+z x^{2} y^{5}$ & $\begin{array}{c}t+z^{3} x+g(z, x, y) \subset \mathbb{P}(95,28,11,9) \\
\mathbb{P}(28,11,9), \text { Diff }=\left(\frac{1}{2}, 0,0,0\right)\end{array}$ & 18 \\
\hline 128 & $x^{7} y^{2}+z^{2} y^{4}$ & $\begin{array}{c}t^{2}+z^{3} x+x^{7} y+z^{2} y^{2} \subset \mathbb{P}(22,13,5,9) \\
\text { Diff }=\left(0,0,0, \frac{1}{2}\right)\end{array}$ & 9 \\
\hline 129 & $x^{6} y^{3}+y^{10}$ & $\begin{array}{c}t^{2}+z x+x^{6} y^{3}+y^{10} \subset \mathbb{P}(30,53,7,6) \\
\text { Diff }=\left(0, \frac{2}{3}, 0,0\right)\end{array}$ & 9 \\
\hline
\end{tabular}


Продолжение таблицы 3

\begin{tabular}{|c|c|c|c|}
\hline 130 & $x^{6} y^{3}+y^{11}$ & $\begin{array}{c}t+z x+x^{6} y^{3}+y^{11} \subset \mathbb{P}(33,29,4,3) \\
\mathbb{P}(29,4,3), \text { Diff }=\left(\frac{1}{2}, \frac{2}{3}, 0,0\right)\end{array}$ & 18 \\
\hline 131 & $x^{6} y^{3}+x^{2} y^{8}$ & $\begin{array}{c}t^{2}+z x+x^{6} y^{3}+x^{2} y^{8} \subset \mathbb{P}(21,37,5,4) \\
\text { Diff }=\left(0, \frac{2}{3}, 0,0\right)\end{array}$ & 12 \\
\hline 132 & $\begin{array}{c}x^{6} y^{3}+z y^{n} \\
n=7 ; 8\end{array}$ & $\begin{array}{c}t+z^{3} x+g(z, x, y) \subset \mathbb{P}(18 n+3,5 n+3,3 n-6,13) \\
\operatorname{Diff}=\left(\frac{1}{2}, 0,0,0\right)\end{array}$ & 10,26 \\
\hline 133 & $x^{6} y^{3}+z x^{2} y^{5}$ & $\begin{array}{c}t+z^{3} x+g(z, x, y) \subset \mathbb{P}(75,22,9,7) \\
\mathbb{P}(22,9,7), \text { Diff }=\left(\frac{1}{2}, 0,0,0\right)\end{array}$ & 14 \\
\hline 134 & $x^{6} y^{3}+z^{2} y^{4}$ & $\begin{array}{c}t^{2}+z^{3} x+g(z, x, y) \subset \mathbb{P}(39,23,9,8) \\
\text { Diff }=\varnothing\end{array}$ & 8 \\
\hline 135 & $x^{5} y^{4}+y^{10}$ & $\begin{array}{c}t^{2}+z x+g(x, y) \subset \mathbb{P}(25,44,6,5) \\
\text { Diff }=\left(0, \frac{2}{3}, 0,0\right)\end{array}$ & 9 \\
\hline 136 & $x^{5} y^{4}+y^{11}$ & $\begin{array}{c}t+z^{3} x+g(x, y) \subset \mathbb{P}(55,16,7,5) \\
\mathbb{P}(16,7,5), \text { Diff }=\left(\frac{1}{2}, 0,0,0\right)\end{array}$ & 10 \\
\hline 137 & $x^{5} y^{4}+y^{12}$ & $\begin{array}{c}t^{2}+z x+x^{5} y^{2}+y^{6} \subset \mathbb{P}(15,26,4,5) \\
\text { Diff }=\left(0, \frac{2}{3}, 0, \frac{1}{2}\right)\end{array}$ & 15 \\
\hline 138 & $x^{5} y^{4}+y^{13}$ & $\begin{array}{c}t+z x+g(x, y) \subset \mathbb{P}(65,56,9,5) \\
\mathbb{P}(56,9,5), \text { Diff }=\left(\frac{1}{2}, \frac{2}{3}, 0,0\right)\end{array}$ & 30 \\
\hline 139 & $x^{5} y^{4}+x^{2} y^{9}$ & $\begin{array}{c}t+z x+g(x, y) \subset \mathbb{P}(37,32,5,3) \\
\mathbb{P}(32,5,3), \text { Diff }=\left(\frac{1}{2}, \frac{2}{3}, 0,0\right)\end{array}$ & 18 \\
\hline 140 & $\begin{array}{c}x^{5} y^{4}+z y^{n} \\
n=7 ; 9\end{array}$ & $\begin{array}{c}t+z^{3} x+g(z, x, y) \subset \mathbb{P}(15 n+4,4 n+4,3 n-8,11) \\
\mathbb{P}(4 n+4,3 n-8,11), \text { Diff }=\left(\frac{1}{2}, 0,0,0\right)\end{array}$ & 16,22 \\
\hline 141 & $x^{5} y^{4}+z y^{8}$ & $\begin{array}{c}t^{2}+z^{3} x+x^{5} y^{2}+z y^{4} \subset \mathbb{P}(31,18,8,11) \\
\text { Diff }=\left(0,0,0, \frac{1}{2}\right)\end{array}$ & 11 \\
\hline 142 & $a z x^{6}+b x^{5} y^{4}+z^{2} y^{4}$ & $\begin{array}{c}t^{2}+z^{3} x+g\left(z, x, y^{1 / 2}\right) \subset \mathbb{P}(17,10,4,7) \\
\text { Diff }=\left(0,0,0, \frac{1}{2}\right)\end{array}$ & 7 \\
\hline 143 & $x^{5} y^{4}+z^{2} y^{5}$ & $\begin{array}{c}t+z^{3} x+g(z, x, y) \subset \mathbb{P}(83,24,11,7) \\
\mathbb{P}(24,11,7), \text { Diff }=\left(\frac{1}{2}, 0,0,0\right)\end{array}$ & 14 \\
\hline 144 & $\begin{aligned} & z x^{4}+y^{2 n} \\
n= & 4,5 ; 6,7 ; 8,9,10\end{aligned}$ & $\begin{array}{c}t^{2}+z^{3} x+z x^{4}+y^{2} \subset \mathbb{P}(11,6,4,11) \\
\text { Diff }=\left(0,0,0, \frac{n-1}{n}\right)\end{array}$ & $5,7,11$ \\
\hline 145 & $\begin{array}{c}z x^{4}+y^{n}, n=7 \\
9 ; 13 ; 15 ; 17 ; 19,21\end{array}$ & $\begin{array}{l}t+z^{3} x+z x^{4}+y \subset \mathbb{P}(11,3,2,11) \\
\mathbb{P}(3,2,11), \text { Diff }=\left(\frac{1}{2}, 0,0, \frac{n-1}{n}\right)\end{array}$ & $\begin{array}{l}7,10,14, \\
16,18,22\end{array}$ \\
\hline 146 & $\begin{array}{c}z x^{4}+x^{2} y^{2 n} \\
n=4,5,6\end{array}$ & $\begin{array}{c}t^{2}+z^{3} x+z x^{4}+x^{2} y^{2} \subset \mathbb{P}(11,6,4,7) \\
\text { Diff }=\left(0,0,0, \frac{n-1}{n}\right)\end{array}$ & 7 \\
\hline 147 & $\begin{array}{l}z x^{4}+x^{2} y^{n} \\
n=9 ; 11,13\end{array}$ & $\begin{array}{c}t+z^{3} x+z x^{4}+x^{2} y \subset \mathbb{P}(11,3,2,7) \\
\mathbb{P}(3,2,7), \text { Diff }=\left(\frac{1}{2}, 0,0, \frac{n-1}{n}\right)\end{array}$ & 10,14 \\
\hline 148 & $\begin{array}{c}z x^{4}+a z^{2} y^{n}+b x^{3} y^{n} \\
n=3 \quad a b \neq 0 ; 7,9\end{array}$ & $\begin{array}{c}t+z^{3} x+g\left(z, x, y^{1 / n}\right) \subset \mathbb{P}(11,3,2,5) \\
\mathbb{P}(3,2,5), \text { Diff }=\left(\frac{1}{2}, 0,0, \frac{n-1}{n}\right)\end{array}$ & 4,10 \\
\hline
\end{tabular}




\begin{tabular}{|c|c|c|c|}
\hline 149 & $\begin{array}{c}z x^{4}+a z^{2} y^{2 n}+b x^{3} y^{2 n} \\
n=2 a b \neq 0,3 a \neq 0 ; 4\end{array}$ & $\begin{array}{c}t^{2}+z^{3} x+g\left(z, x, y^{1 / n}\right) \subset \mathbb{P}(11,6,4,5) \\
\text { Diff }=\left(0,0,0, \frac{n-1}{n}\right)\end{array}$ & 4,5 \\
\hline 150 & $\begin{array}{c}z x^{4} y+y^{2 n} \\
n=4 ; 5,6 ; 7,8\end{array}$ & $\begin{array}{c}t^{2}+z^{3} x+g(z, x, y) \subset \mathbb{P}(11 n, 6 n+1,4 n-3,11) \\
\text { Diff }=\varnothing\end{array}$ & $5,7,11$ \\
\hline 151 & $\begin{aligned} & z x^{4} y+y^{n} \\
n= & 9 ; 11 ; 13 ; 15 ; 17\end{aligned}$ & $\begin{array}{c}t+z^{3} x+g(z, x, y) \subset \mathbb{P}(11 n, 3 n+1,2 n-3,11) \\
\mathbb{P}(3 n+1,2 n-3,11), \text { Diff }=\left(\frac{1}{2}, 0,0,0\right)\end{array}$ & $\begin{array}{c}10,14,16, \\
18,22\end{array}$ \\
\hline 152 & $\begin{array}{c}z x^{4} y+x^{2} y^{2 n} \\
n=4,5\end{array}$ & $\begin{array}{c}t^{2}+z^{3} x+g(z, x, y) \subset \mathbb{P}(11 n-3,6 n-1,4 n-3,7) \\
\text { Diff }=\varnothing\end{array}$ & 7 \\
\hline 153 & $\begin{array}{c}z x^{4} y+x^{2} y^{n} \\
n=7 ; 9 ; 11\end{array}$ & $\begin{array}{c}t+z^{3} x+g(z, x, y) \subset \mathbb{P}(11 n-6,3 n-1,2 n-3,7) \\
\mathbb{P}(3 n-1,2 n-3,7), \text { Diff }=\left(\frac{1}{2}, 0,0,0\right)\end{array}$ & $8,10,14$ \\
\hline 154 & $z x^{4} y+x^{3} y^{7}$ & $\begin{array}{c}t^{2}+z^{3} x+g(z, x, y) \subset \mathbb{P}(34,19,11,5) \\
\text { Diff }=\varnothing\end{array}$ & 5 \\
\hline 155 & $z x^{4} y+x^{3} y^{8}$ & $\begin{array}{c}t+z^{3} x+g(z, x, y) \subset \mathbb{P}(79,22,13,5) \\
\mathbb{P}(22,13,5), \text { Diff }=\left(\frac{1}{2}, 0,0,0\right)\end{array}$ & 10 \\
\hline 156 & $\begin{array}{l}z x^{6}+y^{n} \\
n=9 ; 11\end{array}$ & $\begin{array}{l}t+z^{3} x+z x^{6}+y \subset \mathbb{P}(17,5,2,17) \\
\mathbb{P}(5,2,17), \text { Diff }=\left(\frac{1}{2}, 0,0, \frac{n-1}{n}\right)\end{array}$ & 10,34 \\
\hline 157 & $z x^{6}+y^{10}$ & $\begin{array}{c}t^{2}+z^{3} x+z x^{6}+y^{2} \subset \mathbb{P}(17,10,4,17) \\
\text { Diff }=\left(0,0,0, \frac{4}{5}\right)\end{array}$ & 10 \\
\hline 158 & $z x^{6}+x^{2} y^{7}$ & $\begin{array}{c}t+z^{3} x+z x^{6}+x^{2} y \subset \mathbb{P}(17,5,2,13) \\
\mathbb{P}(5,2,13), \text { Diff }=\left(\frac{1}{2}, 0,0, \frac{6}{7}\right)\end{array}$ & 14 \\
\hline 159 & $z x^{6}+x^{2} y^{8}$ & $\begin{array}{c}t^{2}+z^{3} x+z x^{6}+x^{2} y^{2} \subset \mathbb{P}(17,10,4,13) \\
\text { Diff }=\left(0,0,0, \frac{3}{4}\right)\end{array}$ & 13 \\
\hline 160 & $z x^{6}+x^{3} y^{7}$ & $\begin{array}{c}t+z^{3} x+z x^{6}+x^{3} y \subset \mathbb{P}(17,5,2,11) \\
\mathbb{P}(5,2,11), \text { Diff }=\left(\frac{1}{2}, 0,0, \frac{6}{7}\right)\end{array}$ & 22 \\
\hline 161 & $\begin{array}{l}z x^{5} y+y^{n} \\
n=9 ; 10 ; 11\end{array}$ & $\begin{array}{c}t^{2}+z^{3} x+g(z, x, y) \subset \mathbb{P}(7 n, 4 n+1,2 n-3,14) \\
\text { Diff }=\varnothing\end{array}$ & $5,7,14$ \\
\hline 162 & $\begin{array}{c}z x^{5} y+x^{2} y^{n} \\
n=7 ; 8\end{array}$ & $\begin{array}{c}t^{2}+z^{3} x+g(z, x, y) \subset \mathbb{P}(7 n-3,4 n-1,2 n-3,10) \\
\text { Diff }=\varnothing\end{array}$ & 5,10 \\
\hline 163 & $z x^{5} y+z^{2} y^{4}+a x^{4} y^{5}$ & $\begin{array}{c}t^{2}+z^{3} x+g(z, x, y) \subset \mathbb{P}(29,17,7,6) \\
\text { Diff }=\varnothing\end{array}$ & 6 \\
\hline 164 & $\begin{array}{l}z x^{4} y^{2}+y^{n} \\
n=9 ; 11 ; 13\end{array}$ & $\begin{array}{c}t+z^{3} x+g(z, x, y) \subset \mathbb{P}(11 n, 3 n+2,2 n-6,11) \\
\mathbb{P}(3 n+2,2 n-6,11), \text { Diff }=\left(\frac{1}{2}, 0,0,0\right)\end{array}$ & $14,16,22$ \\
\hline 165 & $z x^{4} y^{2}+y^{10}$ & $\begin{array}{c}t^{2}+z^{3} x+g(z, x, y) \subset \mathbb{P}(55,32,14,11) \\
\text { Diff }=\varnothing\end{array}$ & 7 \\
\hline 166 & $z x^{4} y^{2}+y^{12}$ & $\begin{array}{c}t^{2}+z^{3} x+z x^{4} y+y^{6} \subset \mathbb{P}(33,19,9,11) \\
\text { Diff }=\left(0,0,0, \frac{1}{2}\right)\end{array}$ & 11 \\
\hline 167 & $\begin{array}{c}z x^{4} y^{2}+x^{2} y^{n} \\
n=7 ; 9\end{array}$ & $\begin{array}{c}t+z^{3} x+g(z, x, y) \subset \mathbb{P}(11 n-12,3 n-2,2 n-6,7) \\
\mathbb{P}(3 n-2,2 n-6,7), \text { Diff }=\left(\frac{1}{2}, 0,0,0\right)\end{array}$ & 10,14 \\
\hline
\end{tabular}


Продолжение таблицы 3

\begin{tabular}{|c|c|c|c|}
\hline 168 & $z x^{4} y^{2}+x^{2} y^{8}$ & $t^{2}+z^{3} x+z x^{4} y+x^{2} y^{4} \subset \mathbb{P}(19,11,5,7)$ & 7 \\
& & $\operatorname{Diff}=\left(0,0,0, \frac{1}{2}\right)$ & \\
\hline 169 & $z x^{4} y^{2}+a z^{2} y^{5}+b x^{3} y^{7}$ & $t+z^{3} x+g(z, x, y) \subset \mathbb{P}(59,17,8,5)$ & 10 \\
& & $\mathbb{P}(17,8,5), \operatorname{Diff}=\left(\frac{1}{2}, 0,0,0\right)$ & \\
\hline
\end{tabular}

(1) Разобрано в примере 3.23.

(2) Особенность:

$$
t^{2}+z^{3} x+\underline{a} z x^{i}\left(x+y^{2}\right)^{j} f_{4-i-j}\left(x, y^{2}\right)+b z^{2} y^{5}+\underline{c} x^{k} y\left(x+y^{2}\right)^{l} f_{5-k-l}\left(x, y^{2}\right) .
$$

Условия на исключительность: $b \neq 0||(b=0 \&(i \leqslant 1|| k \leqslant 2) \&(j \leqslant 2|| l \leqslant 4))$.

(3) Особенность:

$t^{2}+z^{3} x+\underline{a} z x^{i} y^{j}(x+y)^{k} f_{5-i-j-k}(x, y)+\underline{b} x^{l} y^{m}(x+y)^{n} f_{7-l-m-n}(x, y)+c z^{2} y^{3}$.

Общие условия на исключительность для двух случаев: $(c \neq 0) \|((i=0 \| l=0) \&$ $(k \leqslant 2|| n \leqslant 2))$. Первый случай $j \leqslant 1$ и коэффициент $\underline{a}$ опущен (см. комментарий к таблицам). Второй случай $m \leqslant 2$ и коэффициент $\underline{b}$ опущен.

ТАБЛИЦА 4. Особенность: $t^{2}+z^{3} y+g(z, x, y)$

\begin{tabular}{|c|c|c|c|}
\hline 1 & $\begin{array}{c}x^{7}+y^{2 n} \\
n=4 ; 6 ; 9,10 ; 12,13\end{array}$ & $\begin{array}{c}t^{2}+z y+x+y^{2 n} \subset \mathbb{P}(n, 2 n-1,2 n, 1) \\
\mathbb{P}(n, 2 n-1,1), \text { Diff }=\left(0, \frac{2}{3}, \frac{6}{7}, 0\right)\end{array}$ & $\begin{array}{c}7,9,15 \\
21\end{array}$ \\
\hline 2 & $\begin{array}{c}x^{7}+y^{n} \\
n=9 ; 11 ; 15 ; 17 ; 23 ; 27\end{array}$ & $\begin{array}{l}t+z y+x+y^{n} \subset \mathbb{P}(n, n-1, n, 1) \\
\mathbb{P}(n-1, n, 1), \text { Diff }=\left(\frac{1}{2}, \frac{2}{3}, \frac{6}{7}, 0\right)\end{array}$ & $\begin{array}{l}14,16,22 \\
28,36,42\end{array}$ \\
\hline 3 & $\begin{array}{c}a y^{2 n} z^{i} f_{2-i}\left(z, y^{2 n-1}\right)+x^{7} \\
n=2,3,4\end{array}$ & $\begin{array}{c}t^{2}+z^{3} y+g\left(z, x^{1 / 7}, y\right) \subset \mathbb{P}(3 n-1,2 n-1,6 n-2,1) \\
\mathbb{P}(3 n-1,2 n-1,1), \text { Diff }=\left(0,0, \frac{6}{7}, 0\right)\end{array}$ & 7 \\
\hline 4 & $\begin{array}{c}a y^{n} z^{i} f_{2-i}\left(z, y^{n-1}\right)+x^{7} \\
n=5 ; 7,9\end{array}$ & $\begin{array}{c}t+z^{3} y+g\left(z, x^{1 / 7}, y\right) \subset \mathbb{P}(3 n-2, n-1,3 n-2,1) \\
\mathbb{P}(n-1,3 n-2,1), \text { Diff }=\left(\frac{1}{2}, 0, \frac{6}{7}, 0\right)\end{array}$ & 8,14 \\
\hline 5 & $\begin{array}{c}x^{i} f_{7-i}\left(x, y^{2}\right) \\
i \leqslant 4\end{array}$ & $\begin{array}{c}t^{2}+z y+g(x, y) \subset \mathbb{P}(7,13,2,1) \\
\text { Diff }=\left(0, \frac{2}{3}, 0,0\right)\end{array}$ & 3 \\
\hline 6 & $\begin{array}{c}x^{i} f_{7-i}\left(x, y^{3}\right) \\
i \leqslant 5\end{array}$ & $\begin{array}{c}t+z y+g(x, y) \subset \mathbb{P}(21,20,3,1) \\
\mathbb{P}(20,3,1), \text { Diff }=\left(\frac{1}{2}, \frac{2}{3}, 0,0\right)\end{array}$ & 6 \\
\hline 7 & $\begin{array}{l}\quad x^{7}+x y^{n} \\
n=7 ; 11 ; 13 ; 17,19 ; 23\end{array}$ & $\begin{array}{c}t+z y+x^{7}+x y^{n} \subset \mathbb{P}(7 n, 7 n-6, n, 6) \\
\mathbb{P}(7 n-6, n, 6), \text { Diff }=\left(\frac{1}{2}, \frac{2}{3}, 0,0\right)\end{array}$ & $\begin{array}{c}10,16,22 \\
30,36\end{array}$ \\
\hline 8 & $\begin{array}{l}x^{3 i+1} f_{2-i}\left(x^{3}, y^{2 n}\right) \\
n=2 i=0 ;(4,5) i \leqslant 1\end{array}$ & $\begin{array}{c}t^{2}+z y+g(x, y) \subset \mathbb{P}(7 n, 14 n-3,2 n, 3) \\
\text { Diff }=\left(0, \frac{2}{3}, 0,0\right)\end{array}$ & 3,9 \\
\hline 9 & $\begin{array}{c}x^{3 i+1} f_{2-i}\left(x^{3}, y^{n}\right) \\
n=5,7 ; 11\end{array}$ & $\begin{array}{c}t+z y+g(x, y) \subset \mathbb{P}(7 n, 7 n-3, n, 3) \\
\mathbb{P}(7 n-3, n, 3), \text { Diff }=\left(\frac{1}{2}, \frac{2}{3}, 0,0\right)\end{array}$ & 12,18 \\
\hline 10 & $\begin{array}{c}x^{2 i+1} f_{3-i}\left(x^{2}, y^{n}\right) \\
n=3 i \leqslant 1 ; 7 i \leqslant 2\end{array}$ & $\begin{array}{c}t+z y+g(x, y) \subset \mathbb{P}(7 n, 7 n-2, n, 2) \\
\mathbb{P}(7 n-2, n, 2), \text { Diff }=\left(\frac{1}{2}, \frac{2}{3}, 0,0\right)\end{array}$ & 6,12 \\
\hline 11 & $\begin{array}{c}a z y^{2} x^{2 j} f_{2-j}\left(x^{2}, y^{5}\right)+ \\
x^{2 i+1} f_{3-i}\left(x^{2}, y^{5}\right) \\
\end{array}$ & $\begin{array}{c}t+z^{3} y+g(z, x, y) \subset \mathbb{P}(35,11,5,2) \\
\mathbb{P}(11,5,2), \text { Diff }=\left(\frac{1}{2}, 0,0,0\right)\end{array}$ & 4 \\
\hline 12 & $\begin{array}{l}x^{7}+x^{2} y^{2 n} \\
n=3,6 ; 8,9\end{array}$ & $\begin{array}{c}t^{2}+z y+g(x, y) \subset \mathbb{P}(7 n, 14 n-5,2 n, 5) \\
\text { Diff }=\left(0, \frac{2}{3}, 0,0\right)\end{array}$ & 9,15 \\
\hline
\end{tabular}


Продолжение таблицы 4

\begin{tabular}{|c|c|c|c|}
\hline 13 & $\begin{array}{c}x^{7}+x^{2} y^{n} \\
n=7 ; 9 ; 13 ; 19\end{array}$ & $\begin{array}{c}t+z y+g(x, y) \subset \mathbb{P}(7 n, 7 n-5, n, 5) \\
\mathbb{P}(7 n-5, n, 5), \text { Diff }=\left(\frac{1}{2}, \frac{2}{3}, 0,0\right)\end{array}$ & $\begin{array}{c}10,16,18 \\
30\end{array}$ \\
\hline 14 & $\begin{array}{c}a z x^{3} y^{2 n+1}+x^{7}+b x^{2} y^{6 n+2} \\
n=1 \quad b \neq 0 ; 2\end{array}$ & $\begin{array}{c}t^{2}+z^{3} y+g(z, x, y) \subset \mathbb{P}(21 n+7,14 n+3,6 n+2,5) \\
\text { Diff }=\varnothing\end{array}$ & 4,5 \\
\hline 15 & $\begin{array}{c}a z x^{3} y^{n}+x^{7}+b x^{2} y^{3 n-1} \\
n=4,6\end{array}$ & $\begin{array}{c}t+z^{3} y+g(z, x, y) \subset \mathbb{P}(21 n-7,7 n-4,3 n-1,5) \\
\mathbb{P}(7 n-4,3 n-1,5), \text { Diff }=\left(\frac{1}{2}, 0,0,0\right)\end{array}$ & 10 \\
\hline 16 & $\begin{array}{c}x^{7}+x^{3} y^{n} \\
n=5 ; 9,11 ; 15\end{array}$ & $\begin{array}{c}t+z y+g(x, y) \subset \mathbb{P}(7 n, 7 n-4, n, 4) \\
\mathbb{P}(7 n-4, n, 4), \text { Diff }=\left(\frac{1}{2}, \frac{2}{3}, 0,0\right)\end{array}$ & $10,18,24$ \\
\hline 17 & $\begin{array}{l}a z x^{2} y^{2 n-1}+x^{7}+ \\
+b x^{3} y^{3 n-2}, n=3,5\end{array}$ & $\begin{array}{c}t+z^{3} y+g(z, x, y) \subset \mathbb{P}(21 n-14,7 n-6,3 n-2,4) \\
\mathbb{P}(7 n-6,3 n-2,4), \text { Diff }=\left(\frac{1}{2}, 0,0,0\right)\end{array}$ & 8 \\
\hline 18 & $\begin{array}{c}x^{7}+z y^{n} \\
n=6 ; 8,10 ; 14 ; 16,18\end{array}$ & $\begin{array}{l}t+z^{3} y+x+z y^{n} \subset \mathbb{P}(3 n-1, n-1,3 n-1,2) \\
\quad \mathbb{P}(n-1,3 n-1,2), \text { Diff }=\left(\frac{1}{2}, 0, \frac{6}{7}, 0\right)\end{array}$ & $\begin{array}{c}8,14,22 \\
28\end{array}$ \\
\hline 19 & $\begin{array}{l}x^{7}+z x y^{2 n+1} \\
n=2,3 ; 4,5,6\end{array}$ & $\begin{array}{c}t^{2}+z^{3} y+g(z, x, y) \subset \mathbb{P}(21 n+7,14 n+1,6 n+2,11) \\
\text { Diff }=\varnothing\end{array}$ & 5,11 \\
\hline 20 & $\begin{array}{c}\quad x^{7}+z x y^{n} \\
n=6 ; 8,10 ; 12,14\end{array}$ & $\begin{array}{c}t+z^{3} y+g(z, x, y) \subset \mathbb{P}(21 n-7,7 n-6,3 n-1,11) \\
\mathbb{P}(7 n-6,3 n-1,11), \text { Diff }=\left(\frac{1}{2}, 0,0,0\right)\end{array}$ & $10,16,22$ \\
\hline 21 & $\begin{array}{l}x^{7}+z x^{2} y^{n} \\
n=4,6 ; 8,10\end{array}$ & $\begin{array}{c}t+z^{3} y+g(z, x, y) \subset \mathbb{P}(21 n-7,7 n-5,3 n-1,8) \\
\mathbb{P}(7 n-5,3 n-1,8), \text { Diff }=\left(\frac{1}{2}, 0,0,0\right)\end{array}$ & 10,16 \\
\hline 22 & $\begin{array}{c}x^{8}+y^{n} \\
n=9 ; 11 ; 15\end{array}$ & $\begin{array}{c}t^{2}+z y+x^{2}+y^{n} \subset \mathbb{P}(n, 2 n-2, n, 2) \\
\text { Diff }=\left(0, \frac{2}{3}, \frac{3}{4}, 0\right)\end{array}$ & $13,16,24$ \\
\hline 23 & $\begin{array}{c}z y^{2}\left(b x^{4}+c y^{5}\right)+x^{8}+d x^{4} y^{5}+ \\
e y^{10},|c|+|d|+|e| \neq 0\end{array}$ & $\begin{array}{c}t^{2}+z^{3} y+g\left(z, x^{1 / 4}, y\right) \subset \mathbb{P}(5,3,5,1) \\
\text { Diff }=\left(0,0, \frac{3}{4}, 0\right)\end{array}$ & 4 \\
\hline 24 & $\begin{array}{c}x^{2 i} f_{4-i}\left(x^{2}, y^{3}\right) \\
i \leqslant 2\end{array}$ & $\begin{array}{c}t^{2}+z y+g\left(x^{1 / 2}, y\right) \subset \mathbb{P}(6,11,3,1) \\
\text { Diff }=\left(0, \frac{2}{3}, \frac{1}{2}, 0\right)\end{array}$ & 6 \\
\hline 25 & $y^{5} z^{i} f_{2-i}\left(z, y^{4}\right)+x^{8}$ & $\begin{array}{c}t^{2}+z^{3} y+g\left(z, x^{1 / 4}, y\right) \subset \mathbb{P}(13,8,13,2) \\
\text { Diff }=\left(0,0, \frac{3}{4}, 0\right)\end{array}$ & 8 \\
\hline 26 & $x^{8}+a x^{4} y^{7}+b y^{14}$ & $\begin{array}{c}t^{2}+z y+x^{2}+a x y^{7}+b y^{14} \subset \mathbb{P}(7,13,7,1) \\
\text { Diff }=\left(0, \frac{2}{3}, \frac{3}{4}, 0\right)\end{array}$ & 12 \\
\hline 27 & $\begin{array}{c}a z x^{3} y^{n}+x^{8}+b x y^{3 n-1} \\
n=3 \quad b \neq 0 ; 4\end{array}$ & $\begin{array}{c}t^{2}+z^{3} y+g(z, x, y) \subset \mathbb{P}(12 n-4,8 n-5,3 n-1,7) \\
\text { Diff }=\varnothing\end{array}$ & 4,7 \\
\hline 28 & $\begin{array}{c}\quad x^{8}+x y^{n} \\
n=9 ; 10 ; 12 ; 13\end{array}$ & $\begin{array}{c}t^{2}+z y+x^{8}+x y^{n} \subset \mathbb{P}(4 n, 8 n-7, n, 7) \\
\text { Diff }=\left(0, \frac{2}{3}, 0,0\right)\end{array}$ & $\begin{array}{c}13,15,18 \\
21\end{array}$ \\
\hline 29 & $\begin{array}{c}x^{8}+x^{2} y^{n} \\
n=7 ; 11\end{array}$ & $\begin{array}{c}t^{2}+z y+x^{4}+x y^{n} \subset \mathbb{P}(2 n, 4 n-3, n, 3) \\
\text { Diff }=\left(0, \frac{2}{3}, \frac{1}{2}, 0\right)\end{array}$ & 10,18 \\
\hline 30 & $\begin{array}{c}x^{3 i+2} f_{2-i}\left(x^{3}, y^{n}\right) \\
n=4 i=0 ; 5 i \leqslant 1\end{array}$ & $\begin{array}{c}t^{2}+z y+g(x, y) \subset \mathbb{P}(4 n, 8 n-3, n, 3) \\
\operatorname{Diff}=\left(0, \frac{2}{3}, 0,0\right)\end{array}$ & 6,9 \\
\hline 31 & $\begin{array}{c}x^{8}+x^{3} y^{n} \\
n=6 ; 8 ; 9\end{array}$ & $\begin{array}{c}t^{2}+z y+g(x, y) \subset \mathbb{P}(4 n, 8 n-5, n, 5) \\
\operatorname{Diff}=\left(0, \frac{2}{3}, 0,0\right)\end{array}$ & $9,12,15$ \\
\hline
\end{tabular}


Продолжение таблицы 4

\begin{tabular}{|c|c|c|c|}
\hline 32 & $b z x^{2} y^{5}+x^{8}+c x^{3} y^{7}$ & $\begin{array}{c}t^{2}+z^{3} y+g(z, x, y) \subset \mathbb{P}(28,17,7,5) \\
\text { Diff }=\varnothing\end{array}$ & 5 \\
\hline 33 & $\begin{array}{l}x^{8}+z y^{n} \\
n=6 ; 8 ; 10\end{array}$ & $\begin{array}{c}t^{2}+z^{3} y+x^{2}+z y^{n} \subset \mathbb{P}(3 n-1,2 n-2,3 n-1,4) \\
\text { Diff }=\left(0,0, \frac{3}{4}, 0\right)\end{array}$ & $10,13,16$ \\
\hline 34 & $\begin{array}{l}x^{8}+z x y^{n} \\
n=5 ; 6,7 ; 8\end{array}$ & $\begin{array}{c}t^{2}+z^{3} y+g(z, x, y) \subset \mathbb{P}(12 n-4,8 n-7,3 n-1,13) \\
\text { Diff }=\varnothing\end{array}$ & $7,10,13$ \\
\hline 35 & $\begin{array}{c}x^{8}+z x^{2} y^{n} \\
n=4 ; 6\end{array}$ & $\begin{array}{c}t^{2}+z^{3} y+x^{4}+z x y^{n} \subset \mathbb{P}(6 n-2,4 n-3,3 n-1,5) \\
\text { Diff }=\left(0,0, \frac{1}{2}, 0\right)\end{array}$ & 7,10 \\
\hline 36 & $\begin{array}{c}\underline{a} z^{2} x^{3}+b z y^{2} x^{2 i} f_{2-i}\left(x^{2}, y^{3}\right)+ \\
\underline{c} x^{2 j+1} y f_{3-j}\left(x^{2}, y^{3}\right) \\
i \leqslant 1|| j \leqslant 1\end{array}$ & $\begin{array}{c}t+z^{3} y+g(z, x, y) \subset \mathbb{P}(23,7,3,2) \\
\mathbb{P}(7,3,2), \text { Diff }=\left(\frac{1}{2}, 0,0,0\right)\end{array}$ & 4 \\
\hline 37 & $y^{4} z^{i} f_{2-i}\left(z, y^{3}\right)+x^{9}$ & $\begin{array}{c}t^{2}+z^{3} y+g\left(z, x^{1 / 9}, y\right) \subset \mathbb{P}(5,3,10,1) \\
\mathbb{P}(5,3,1), \text { Diff }=\left(0,0, \frac{8}{9}, 0\right)\end{array}$ & 9 \\
\hline 38 & $x^{9}+a z x^{3} y^{4}+b y^{11}$ & $\begin{array}{c}t+z^{3} y+g\left(z, x^{1 / 3}, y\right) \subset \mathbb{P}(33,10,11,3) \\
\mathbb{P}(10,11,3), \text { Diff }=\left(\frac{1}{2}, 0, \frac{2}{3}, 0\right)\end{array}$ & 18 \\
\hline 39 & $x^{9}+x y^{9}$ & $\begin{array}{c}t+z y+g(x, y) \subset \mathbb{P}(81,73,9,8) \\
\mathbb{P}(73,9,8), \text { Diff }=\left(\frac{1}{2}, \frac{2}{3}, 0,0\right)\end{array}$ & 30 \\
\hline 40 & $x^{9}+a x^{5} y^{5}+b x y^{10}$ & $\begin{array}{c}t+z y+g(x, y) \subset \mathbb{P}(45,41,5,4) \\
\mathbb{P}(41,5,4), \text { Diff }=\left(\frac{1}{2}, \frac{2}{3}, 0,0\right)\end{array}$ & 24 \\
\hline 41 & $x^{9}+x^{2} y^{8}$ & $\begin{array}{c}t^{2}+z y+g(x, y) \subset \mathbb{P}(36,65,8,7) \\
\operatorname{Diff}=\left(0, \frac{2}{3}, 0,0\right)\end{array}$ & 12 \\
\hline 42 & $x^{9}+x^{2} y^{9}$ & $\begin{array}{c}t+z y+g(x, y) \subset \mathbb{P}(81,74,9,7) \\
\mathbb{P}(74,9,7), \text { Diff }=\left(\frac{1}{2}, \frac{2}{3}, 0,0\right)\end{array}$ & 42 \\
\hline 43 & $\begin{array}{c}b z x^{2} y^{5}+ \\
+x^{9}+c x^{3} y^{7}\end{array}$ & $\begin{array}{c}t+z^{3} y+g(z, x, y) \subset \mathbb{P}(63,19,7,6) \\
\mathbb{P}(19,7,6), \text { Diff }=\left(\frac{1}{2}, 0,0,0\right)\end{array}$ & 12 \\
\hline 44 & $x^{9}+x^{4} y^{6}$ & $\begin{array}{c}t^{2}+z y+g(x, y) \subset \mathbb{P}(27,49,6,5) \\
\text { Diff }=\left(0, \frac{2}{3}, 0,0\right)\end{array}$ & 15 \\
\hline 45 & $x^{9}+z y^{8}$ & $\begin{array}{c}t+z^{3} y+x+z y^{8} \subset \mathbb{P}(23,7,23,2) \\
\mathbb{P}(7,23,2), \text { Diff }=\left(\frac{1}{2}, 0, \frac{8}{9}, 0\right)\end{array}$ & 36 \\
\hline 46 & $x^{9}+z x y^{6}$ & $\begin{array}{c}t+z^{3} y+g(z, x, y) \subset \mathbb{P}(153,46,17,15) \\
\mathbb{P}(46,17,15), \text { Diff }=\left(\frac{1}{2}, 0,0,0\right)\end{array}$ & 10 \\
\hline 47 & $a y^{4} z^{i} f_{2-i}\left(z, y^{3}\right)+x^{7} y^{2}$ & $\begin{array}{c}t^{2}+z^{3} y+g\left(z, x^{1 / 7}, y\right) \subset \mathbb{P}(5,3,8,1) \\
\text { Diff }=\left(0,0, \frac{6}{7}, 0\right)\end{array}$ & 7 \\
\hline 48 & $a y^{5} z^{i} f_{2-i}\left(z, y^{4}\right)+x^{7} y^{2}$ & $\begin{array}{c}t+z^{3} y+g\left(z, x^{1 / 7}, y\right) \subset \mathbb{P}(13,4,11,1) \\
\mathbb{P}(4,11,1), \text { Diff }=\left(\frac{1}{2}, 0, \frac{6}{7}, 0\right)\end{array}$ & 14 \\
\hline 49 & $\begin{array}{l}x^{7} y^{2}+y^{n} \\
n=11 ; 15\end{array}$ & $\begin{array}{l}t+z y+x y^{2}+y^{n} \subset \mathbb{P}(n, n-1, n-2,1) \\
\mathbb{P}(n-1, n-2,1), \text { Diff }=\left(\frac{1}{2}, \frac{2}{3}, \frac{6}{7}, 0\right)\end{array}$ & 28,42 \\
\hline
\end{tabular}




\begin{tabular}{|c|c|c|c|}
\hline 50 & $\begin{array}{c}x^{7} y^{2}+y^{2 n} \\
n=6 ; 7\end{array}$ & $\begin{array}{c}t^{2}+z y+x y^{2}+y^{2 n} \subset \mathbb{P}(n, 2 n-1,2 n-2,1) \\
\text { Diff }=\left(0, \frac{2}{3}, \frac{6}{7}, 0\right)\end{array}$ & 15,21 \\
\hline 51 & $\begin{array}{c}x^{7} y^{2}+x y^{n} \\
n=9 ; 13\end{array}$ & $\begin{array}{c}t+z y+x^{7} y^{2}+x y^{n} \subset \mathbb{P}(7 n-2,7 n-8, n-2,6) \\
\mathbb{P}(7 n-8, n-2,6), \text { Diff }=\left(\frac{1}{2}, \frac{2}{3}, 0,0\right)\end{array}$ & 22,36 \\
\hline 52 & $x^{7} y^{2}+a x^{4} y^{6}+b x y^{10}$ & $\begin{array}{c}t^{2}+z y+g(x, y) \subset \mathbb{P}(17,31,4,3) \\
\text { Diff }=\left(0, \frac{2}{3}, 0,0\right)\end{array}$ & 9 \\
\hline 53 & $\begin{array}{c}y^{2} x^{2 i+1} f_{3-i}\left(x^{2}, y^{3}\right) \\
i \leqslant 2\end{array}$ & $\begin{array}{c}t+z y+g(x, y) \subset \mathbb{P}(25,23,3,2) \\
\mathbb{P}(23,3,2), \text { Diff }=\left(\frac{1}{2}, \frac{2}{3}, 0,0\right)\end{array}$ & 12 \\
\hline 54 & $x^{7} y^{2}+a x^{4} y^{7}+b x y^{12}$ & $\begin{array}{c}t+z y+g(x, y) \subset \mathbb{P}(41,38,5,3) \\
\mathbb{P}(38,5,3), \text { Diff }=\left(\frac{1}{2}, \frac{2}{3}, 0,0\right)\end{array}$ & 18 \\
\hline 55 & $\begin{array}{c}x^{7} y^{2}+x^{2} y^{2 n} \\
n=4 ; 5\end{array}$ & $\begin{array}{c}t^{2}+z y+g(x, y) \subset \mathbb{P}(7 n-2,14 n-9,2 n-2,5) \\
\text { Diff }=\left(0, \frac{2}{3}, 0,0\right)\end{array}$ & 9,15 \\
\hline 56 & $a z x^{3} y^{4}+x^{7} y^{2}+b x^{2} y^{9}$ & $\begin{array}{c}t+z^{3} y+g(z, x, y) \subset \mathbb{P}(59,18,7,5) \\
\mathbb{P}(18,7,5), \text { Diff }=\left(\frac{1}{2}, 0,0,0\right)\end{array}$ & 10 \\
\hline 57 & $x^{7} y^{2}+x^{2} y^{11}$ & $\begin{array}{c}t+z y+g(x, y) \subset \mathbb{P}(73,68,9,5) \\
\mathbb{P}(68,9,5), \text { Diff }=\left(\frac{1}{2}, \frac{2}{3}, 0,0\right)\end{array}$ & 30 \\
\hline 58 & $\begin{array}{c}b z x^{6}+c z x^{2} y^{5}+ \\
+d x^{7} y^{2}+e x^{3} y^{7},|b|+|d| \neq 0 \\
|c|+|e| \neq 0\end{array}$ & $\begin{array}{l}t+z^{3} y+g(z, x, y) \subset \mathbb{P}(43,13,5,4) \\
\mathbb{P}(13,5,4), \text { Diff }=\left(\frac{1}{2}, 0,0,0\right)\end{array}$ & 8 \\
\hline 59 & $x^{7} y^{2}+x^{3} y^{9}$ & $\begin{array}{c}t+z y+g(x, y) \subset \mathbb{P}(57,53,7,4) \\
\mathbb{P}(53,7,4), \text { Diff }=\left(\frac{1}{2}, \frac{2}{3}, 0,0\right)\end{array}$ & 24 \\
\hline 60 & $\begin{array}{c}x^{7} y^{2}+z y^{n} \\
n=8 ; 10\end{array}$ & $\begin{array}{c}t+z^{3} y+x y^{2}+z y^{n} \subset \mathbb{P}(3 n-1, n-1,3 n-5,2) \\
\mathbb{P}(n-1,3 n-5,2), \text { Diff }=\left(\frac{1}{2}, 0, \frac{6}{7}, 0\right)\end{array}$ & 22,28 \\
\hline 61 & $\begin{array}{c}x^{7} y^{2}+z x y^{n} \\
n=6 ; 8\end{array}$ & $\begin{array}{c}t+z^{3} y+g(z, x, y) \subset \mathbb{P}(21 n-13,7 n-8,3 n-5,11) \\
\mathbb{P}(7 n-8,3 n-5,11), \text { Diff }=\left(\frac{1}{2}, 0,0,0\right)\end{array}$ & 16,22 \\
\hline 62 & $x^{7} y^{2}+z x y^{7}$ & $\begin{array}{c}t^{2}+z^{3} y+g(z, x, y) \subset \mathbb{P}(67,41,16,11) \\
\text { Diff }=\varnothing\end{array}$ & 11 \\
\hline 63 & $x^{7} y^{2}+z x^{2} y^{6}$ & $\begin{array}{c}t+z^{3} y+g(z, x, y) \subset \mathbb{P}(107,33,13,8) \\
\mathbb{P}(33,13,8), \text { Diff }=\left(\frac{1}{2}, 0,0,0\right)\end{array}$ & 16 \\
\hline 64 & $\begin{array}{c}z x^{5}+y^{2 n} \\
n=4 ; 7 ; 9,10\end{array}$ & $\begin{array}{c}t^{2}+z^{3} y+z x+y^{2 n} \subset \mathbb{P}(3 n, 2 n-1,4 n+1,3) \\
\text { Diff }=\left(0,0, \frac{4}{5}, 0\right)\end{array}$ & $5,11,15$ \\
\hline 65 & $\begin{array}{c}z x^{5}+y^{n} \\
n=9 ; 11 ; 15 ; 21\end{array}$ & $\begin{array}{c}t+z^{3} y+z x+y^{n} \subset \mathbb{P}(3 n, n-1,2 n+1,3) \\
\mathbb{P}(n-1,2 n+1,3), \text { Diff }=\left(\frac{1}{2}, 0, \frac{4}{5}, 0\right)\end{array}$ & $\begin{array}{c}12,15,20 \\
30\end{array}$ \\
\hline 66 & $\begin{array}{l}a y^{2 n} z^{i} f_{2-i}\left(z, y^{2 n-1}\right)+z x^{5} \\
\quad+d x^{5} y^{2 n-1}, n=2 a \neq 0,3\end{array}$ & $\begin{array}{c}t^{2}+z^{3} y+g\left(z, x^{1 / 5}, y\right) \subset \mathbb{P}(3 n-1,2 n-1,4 n-1,1) \\
\text { Diff }=\left(0,0, \frac{4}{5}, 0\right)\end{array}$ & 5 \\
\hline 67 & $\begin{array}{l}a y^{n} z^{i} f_{2-i}\left(z, y^{n-1}\right)+z x^{5}+ \\
+c x^{5} y^{n-1}, n=5 \quad a \neq 0 ; 7\end{array}$ & $\begin{array}{c}t+z^{3} y+g\left(z, x^{1 / 5}, y\right) \subset \mathbb{P}(3 n-2, n-1,2 n-1,1) \\
\mathbb{P}(n-1,2 n-1,1), \text { Diff }=\left(\frac{1}{2}, 0, \frac{4}{5}, 0\right)\end{array}$ & 6,10 \\
\hline
\end{tabular}


Продолжение таблицы 4

\begin{tabular}{|c|c|c|c|}
\hline 68 & $\begin{array}{c}z x^{5}+b z x^{2} y^{5}+ \\
+e x^{6} y^{2}+c x^{3} y^{7}+d y^{12} \\
|b|+|c|+|d| \neq 0\end{array}$ & $\begin{array}{c}t^{2}+z^{3} y+g(z, x, y) \subset \mathbb{P}(18,11,5,3) \\
\text { Diff }=\varnothing\end{array}$ & 3 \\
\hline 69 & $\begin{array}{c}z x^{5}+b z x^{2} y^{7}+ \\
+e x^{6} y^{3}+c x^{3} y^{10}+d y^{17} \\
|b|+|c|+|d| \neq 0\end{array}$ & $\begin{array}{c}t+z^{3} y+g(z, x, y) \subset \mathbb{P}(51,16,7,3) \\
\mathbb{P}(16,7,3) \text { Diff }=\left(\frac{1}{2}, 0,0,0\right)\end{array}$ & 6 \\
\hline 70 & $\begin{array}{l}z x^{5}+x y^{2 n+1} \\
n=3 ; 4,5,6 ; 7,8\end{array}$ & $\begin{array}{c}t^{2}+z^{3} y+g(z, x, y) \subset \mathbb{P}(15 n+8,10 n+1,4 n+3,13) \\
\text { Diff }=\varnothing\end{array}$ & $5,9,13$ \\
\hline 71 & $\begin{array}{l}z x^{5}+x y^{n} \\
n=8 ; 10 ; 12 ; 14,16 ; 18\end{array}$ & $\begin{array}{c}t+z^{3} y+g(z, x, y) \subset \mathbb{P}(15 n+1,5 n-4,2 n+1,13) \\
\mathbb{P}(5 n-4,2 n+1,13), \text { Diff }=\left(\frac{1}{2}, 0,0,0\right)\end{array}$ & $\begin{array}{c}11,14,16 \\
22,26\end{array}$ \\
\hline 72 & $\begin{array}{l}z x^{5}+x^{2} y^{2 n} \\
n=3,4,5 ; 6,7\end{array}$ & $\begin{array}{c}t^{2}+z^{3} y+g(z, x, y) \subset \mathbb{P}(15 n+1,10 n-3,4 n+1,11) \\
\text { Diff }=\varnothing\end{array}$ & 7,11 \\
\hline 73 & $\begin{array}{c}\quad z x^{5}+x^{2} y^{n} \\
n=7 ; 9 ; 11,13 ; 15\end{array}$ & $\begin{array}{c}t+z^{3} y+g(z, x, y) \subset \mathbb{P}(15 n+2,5 n-3,2 n+1,11) \\
\mathbb{P}(5 n-3,2 n+1,11), \text { Diff }=\left(\frac{1}{2}, 0,0,0\right)\end{array}$ & $\begin{array}{c}12,14,18 \\
22\end{array}$ \\
\hline 74 & $\begin{array}{c}z x^{5}+x^{3} y^{2 n+1} \\
n=2 ; 4,5\end{array}$ & $\begin{array}{c}t^{2}+z^{3} y+g(z, x, y) \subset \mathbb{P}(15 n+9,10 n+3,4 n+3,9) \\
\text { Diff }=\varnothing\end{array}$ & 8,9 \\
\hline 75 & $\begin{array}{c}z x^{5}+x^{3} y^{n} \\
n=6 ; 8 ; 12\end{array}$ & $\begin{array}{c}t+z^{3} y+g(z, x, y) \subset \mathbb{P}(15 n+3,5 n-2,2 n+1,9) \\
\mathbb{P}(5 n-2,2 n+1,9), \text { Diff }=\left(\frac{1}{2}, 0,0,0\right)\end{array}$ & $8,14,18$ \\
\hline 76 & $\begin{array}{c}z x^{5}+x^{4} y^{2 n} \\
\quad n=3,4\end{array}$ & $\begin{array}{c}t^{2}+z^{3} y+g(z, x, y) \subset \mathbb{P}(15 n+2,10 n-1,4 n+1,7) \\
\text { Diff }=\varnothing\end{array}$ & 7 \\
\hline 77 & $\begin{array}{c}z x^{5}+x^{4} y^{n} \\
n=5,7 ; 9\end{array}$ & $\begin{array}{c}t+z^{3} y+g(z, x, y) \subset \mathbb{P}(15 n+4,5 n-1,2 n+1,7) \\
\mathbb{P}(5 n-1,2 n+1,7), \text { Diff }=\left(\frac{1}{2}, 0,0,0\right)\end{array}$ & 10,14 \\
\hline 78 & $\begin{array}{l}z x^{6}+y^{n} \\
n=9,11\end{array}$ & $\begin{array}{c}t^{2}+z^{3} y+z x^{2}+y^{n} \subset \mathbb{P}(3 n, 2 n-2,2 n+1,6) \\
\text { Diff }=\left(0,0, \frac{2}{3}, 0\right)\end{array}$ & 9 \\
\hline 79 & $\begin{array}{c}a y^{4} z^{i} f_{2-i}\left(z, y^{3}\right)+z x^{6}+ \\
+c x^{6} y^{3}\end{array}$ & $\begin{array}{c}t^{2}+z^{3} y+g\left(z, x^{1 / 6}, y\right) \subset \mathbb{P}(5,3,7,1) \\
\text { Diff }=\left(0,0, \frac{5}{6}, 0\right)\end{array}$ & 6 \\
\hline 80 & $z x^{6}+y^{12}$ & $\begin{array}{c}t^{2}+z^{3} y+g\left(z, x^{1 / 6}, y\right) \subset \mathbb{P}(18,11,25,3) \\
\text { Diff }=\left(0,0, \frac{5}{6}, 0\right)\end{array}$ & 18 \\
\hline 81 & $\begin{array}{c}z x^{6}+x y^{n} \\
n=8,9,10 ; 11\end{array}$ & $\begin{array}{c}t+z^{3} y+g(z, x, y) \subset \mathbb{P}(18 n+1,6 n-5,2 n+1,16) \\
\mathbb{P}(6 n-5,2 n+1,16), \text { Diff }=\left(\frac{1}{2}, 0,0,0\right)\end{array}$ & 14,32 \\
\hline 82 & $\begin{array}{c}z x^{6}+x^{2} y^{2 n+1} \\
n=3 ; 4\end{array}$ & $\begin{array}{c}t^{2}+z^{3} y+g\left(z, x^{1 / 2}, y\right) \subset \mathbb{P}(9 n+5,6 n+1,4 n+3,7) \\
\text { Diff }=\left(0,0, \frac{1}{2}, 0\right)\end{array}$ & 5,14 \\
\hline 83 & $z x^{6}+x^{2} y^{8}$ & $\begin{array}{c}t^{2}+z^{3} y+g(z, x, y) \subset \mathbb{P}(73,44,17,14) \\
\text { Diff }=\varnothing\end{array}$ & 7 \\
\hline 84 & $\begin{array}{c}z x^{6}+x^{3} y^{n} \\
n=6 ; 8\end{array}$ & $\begin{array}{c}t+z^{3} y+z x^{2}+x y^{n} \subset \mathbb{P}(6 n+1,2 n-1,2 n+1,4) \\
\mathbb{P}(2 n-1,2 n+1,4), \text { Diff }=\left(\frac{1}{2}, 0, \frac{2}{3}, 0\right)\end{array}$ & 10,24 \\
\hline 85 & $z x^{6}+x^{4} y^{5}$ & $\begin{array}{c}t^{2}+z^{3} y+g(z, x, y) \subset \mathbb{P}(47,28,11,10) \\
\text { Diff }=\varnothing\end{array}$ & 5 \\
\hline
\end{tabular}


Продолжение таблицы 4

\begin{tabular}{|c|c|c|c|}
\hline 86 & $z x^{6}+x^{4} y^{6}$ & $\begin{array}{c}t^{2}+z^{3} y+z x^{3}+x^{2} y^{6} \subset \mathbb{P}(28,17,13,5) \\
\text { Diff }=\left(0,0, \frac{1}{2}, 0\right)\end{array}$ & 10 \\
\hline 87 & $z x^{6}+x^{5} y^{5}$ & $\begin{array}{c}t+z^{3} y+g(z, x, y) \subset \mathbb{P}(95,29,11,8) \\
\mathbb{P}(29,11,8), \text { Diff }=\left(\frac{1}{2}, 0,0,0\right)\end{array}$ & 16 \\
\hline 88 & $\begin{array}{c}z^{2} x^{3}+y^{2 n} \\
n=4 ; 6 ; 7\end{array}$ & $\begin{array}{c}t^{2}+z^{3} y+z^{2} x+y^{2 n} \subset \mathbb{P}(3 n, 2 n-1,2 n+2,3) \\
\text { Diff }=\left(0,0, \frac{2}{3}, 0\right)\end{array}$ & $5,7,9$ \\
\hline 89 & $\begin{array}{l}z^{2} x^{3}+y^{n} \\
n=9 ; 11 ; 15\end{array}$ & $\begin{array}{l}t+z^{3} y+z^{2} x+y^{n} \subset \mathbb{P}(3 n, n-1, n+2,3) \\
\quad \mathbb{P}(n-1, n+2,3), \text { Diff }=\left(\frac{1}{2}, 0, \frac{2}{3}, 0\right)\end{array}$ & $10,12,18$ \\
\hline 90 & $\begin{array}{l}z^{2} x^{3}+z y^{3}\left(b x^{3}+c y^{4}\right)+d x^{6} y^{2} \\
+e x^{3} y^{6}+l y^{10},|c|+|e|+|l| \neq 0\end{array}$ & $\begin{array}{c}t^{2}+z^{3} y+g\left(z, x^{1 / 3}, y\right) \subset \mathbb{P}(5,3,4,1) \\
\text { Diff }=\left(0,0, \frac{2}{3}, 0\right)\end{array}$ & 3 \\
\hline 91 & $\begin{array}{c}z^{2} x^{3}+z y^{4}\left(b x^{3}+c y^{5}\right)+ \\
d x^{6} y^{3}+e x^{3} y^{8}+l y^{13}\end{array}$ & $\begin{array}{c}t+z^{3} y+g\left(z, x^{1 / 3}, y\right) \subset \mathbb{P}(13,4,5,1) \\
\mathbb{P}(4,5,1), \text { Diff }=\left(\frac{1}{2}, 0, \frac{2}{3}, 0\right)\end{array}$ & 6 \\
\hline 92 & $\begin{array}{c}z^{2} x^{3}+x y^{n} \\
n=7 ; 9 ; 11 ; 13\end{array}$ & $\begin{array}{c}t+z^{3} y+z^{2} x^{3}+x y^{n} \subset \mathbb{P}(9 n+2,3 n-2, n+2,8) \\
\mathbb{P}(3 n-2, n+2,8), \text { Diff }=\left(\frac{1}{2}, 0,0,0\right)\end{array}$ & $\begin{array}{c}8,10,14 \\
16\end{array}$ \\
\hline 93 & $\begin{array}{l}z^{2} x^{3}+a z x^{2} y^{n}+b x^{5} y^{n-1}+ \\
+c x y^{2 n}, n=4|a|+|c| \neq 0 ; 6\end{array}$ & $\begin{array}{c}t+z^{3} y+g(z, x, y) \subset \mathbb{P}(9 n+1,3 n-1, n+1,4) \\
\mathbb{P}(3 n-1, n+1,4), \text { Diff }=\left(\frac{1}{2}, 0,0,0\right)\end{array}$ & 6,8 \\
\hline 94 & $\begin{array}{c}z^{2} x^{3}+x^{2} y^{2 n} \\
n=3,4 ; 5\end{array}$ & $\begin{array}{c}t^{2}+z^{3} y+g(z, x, y) \subset \mathbb{P}(9 n+2,6 n-1,2 n+2,7) \\
\text { Diff }=\varnothing\end{array}$ & 5,7 \\
\hline 95 & $\begin{array}{c}z^{2} x^{3}+x^{2} y^{n} \\
n=7 ; 9 ; 11\end{array}$ & $\begin{array}{c}t+z^{3} y+z^{2} x^{3}+x^{2} y^{n} \subset \mathbb{P}(9 n+4,3 n-1, n+2,7) \\
\mathbb{P}(3 n-1, n+2,7), \text { Diff }=\left(\frac{1}{2}, 0,0,0\right)\end{array}$ & $8,12,14$ \\
\hline 96 & $\begin{array}{c}z^{2} x^{3}+a z y^{n}+b x^{3} y^{n-1} \\
n=6 ; 10\end{array}$ & $\begin{array}{c}t+z^{3} y+z^{2} x+z y^{n}+x y^{n-1} \subset \mathbb{P}(3 n-1, n-1, n+1,2) \\
\mathbb{P}(n-1, n+1,2), \operatorname{Diff}=\left(\frac{1}{2}, 0, \frac{2}{3}, 0\right)\end{array}$ & 8,12 \\
\hline 97 & $\begin{array}{c}z^{2} x^{3}+a z x y^{2 n+1}+b x^{4} y^{2 n} \\
n=2 \quad a \neq 0 ; 3\end{array}$ & $\begin{array}{c}t^{2}+z^{3} y+g(z, x, y) \subset \mathbb{P}(9 n+4,6 n+1,2 n+2,5) \\
\text { Diff }=\varnothing\end{array}$ & 3,5 \\
\hline 98 & $\begin{array}{c}z^{2} x^{3}+a z x y^{n+1}+b x^{4} y^{n} \\
n=5 ; 7\end{array}$ & $\begin{array}{c}t+z^{3} y+g(z, x, y) \subset \mathbb{P}(9 n+8,3 n+1, n+2,5) \\
\mathbb{P}(3 n+1, n+2,5), \text { Diff }=\left(\frac{1}{2}, 0,0,0\right)\end{array}$ & 8,10 \\
\hline
\end{tabular}

ТАБлицА 5. Особенность: $t^{2}+z^{5}+g(z, x, y)$

\begin{tabular}{|c|c|c|c|}
\hline 1 & $x^{i} y^{j} f_{6-i-j}(x, y)$ & $t^{2}+z+g(z, x, y) \subset \mathbb{P}(3,6,1,1)$ & 5 \\
& $i \leqslant 2 \& j \leqslant 2$ & $\mathbb{P}(3,1,1)$, Diff $=\left(0, \frac{4}{5}, 0,0\right)$ & \\
\hline 2 & $x^{6}+y^{7}$ & $t^{2}+z+x^{2}+y \subset \mathbb{P}(1,2,1,2)$ & 15 \\
& & $\mathbb{P}(1,1,2)$, Diff $=\left(0, \frac{4}{5}, \frac{2}{3}, \frac{6}{7}\right)$ & \\
\hline 3 & $x^{6}+x y^{6}$ & $t^{2}+z+x^{6}+x y \subset \mathbb{P}(3,6,1,5)$ & 25 \\
& & $\mathbb{P}(3,1,5)$, Diff $=\left(0, \frac{4}{5}, 0, \frac{5}{6}\right)$ & \\
\hline 4 & $x^{6}+z y^{5}$ & $t^{2}+z^{5}+x^{2}+z y \subset \mathbb{P}(5,2,5,8)$ & 6 \\
& & $\operatorname{Diff}=\left(0,0, \frac{2}{3}, \frac{4}{5}\right)$ & \\
\hline 5 & $x^{6}+z x y^{4}$ & $t^{2}+z^{5}+x^{6}+z x y \subset \mathbb{P}(15,6,5,19)$ & 8 \\
& & $\operatorname{Diff}=\left(0,0,0, \frac{3}{4}\right)$ & \\
\hline
\end{tabular}


Продолжение таблицы 5

\begin{tabular}{|c|c|c|c|}
\hline 6 & $x^{6}+z x^{2} y^{3}$ & $\begin{array}{c}t^{2}+z^{5}+x^{6}+z x^{2} y \subset \mathbb{P}(15,6,5,14) \\
\text { Diff }=\left(0,0,0, \frac{2}{3}\right)\end{array}$ & 7 \\
\hline 7 & $a x^{6}+b z x^{3} y^{2}+z^{2} y^{4}$ & $\begin{array}{c}t^{2}+z^{5}+a x^{2}+b z x y+z^{2} y^{2} \subset \mathbb{P}(5,2,5,3) \\
\text { Diff }=\left(0,0, \frac{2}{3}, \frac{1}{2}\right)\end{array}$ & 9 \\
\hline 8 & $x^{6}+z^{2} x y^{3}$ & $\begin{array}{c}t^{2}+z^{5}+x^{6}+z^{2} x y \subset \mathbb{P}(15,6,5,13) \\
\text { Diff }=\left(0,0,0, \frac{2}{3}\right)\end{array}$ & 13 \\
\hline 9 & $\begin{array}{c}a z^{2} x y^{3}+\underline{b} z x^{3} y^{2}+\underline{c} x^{5} y+ \\
d y^{7},|a|+|d| \neq 0\end{array}$ & $\begin{array}{c}t+z^{5}+g(z, x, y) \subset \mathbb{P}(35,7,6,5) \\
\mathbb{P}(7,6,5), \text { Diff }=\left(\frac{1}{2}, 0,0,0\right)\end{array}$ & 10 \\
\hline 10 & $x^{5} y+x y^{6}$ & $\begin{array}{c}t+z+x^{5} y+x y^{6} \subset \mathbb{P}(29,29,5,4) \\
\mathbb{P}(29,5,4), \text { Diff }=\left(\frac{1}{2}, \frac{4}{5}, 0,0\right)\end{array}$ & 30 \\
\hline 11 & $x^{5} y+x^{2} y^{5}$ & $\begin{array}{c}t+z+x^{5} y+x^{2} y^{5} \subset \mathbb{P}(23,23,4,3) \\
\mathbb{P}(23,4,3), \text { Diff }=\left(\frac{1}{2}, \frac{4}{5}, 0,0\right)\end{array}$ & 30 \\
\hline 12 & $x^{5} y+z y^{5}$ & $\begin{array}{l}t+z^{5}+x y+z y^{5} \subset \mathbb{P}(25,5,21,4) \\
\mathbb{P}(5,21,4), \text { Diff }=\left(\frac{1}{2}, 0, \frac{4}{5}, 0\right)\end{array}$ & 30 \\
\hline 13 & $x^{5} y+a z y^{6}+b z^{3} y^{3}$ & $\begin{array}{c}t+z^{5}+x y+a z y^{6}+b z^{3} y^{3} \subset \mathbb{P}(15,3,13,2) \\
\mathbb{P}(3,13,2), \text { Diff }=\left(\frac{1}{2}, 0, \frac{4}{5}, 0\right)\end{array}$ & 20 \\
\hline 14 & $x^{5} y+z x y^{4}$ & $\begin{array}{c}t+z^{5}+x^{5} y+z x y^{4} \subset \mathbb{P}(95,19,16,15) \\
\mathbb{P}(19,16,15), \text { Diff }=\left(\frac{1}{2}, 0,0,0\right)\end{array}$ & 6 \\
\hline 15 & $x^{5} y+z x^{2} y^{3}$ & $\begin{array}{c}t+z^{5}+x^{5} y+z x^{2} y^{3} \subset \mathbb{P}(65,13,11,10) \\
\mathbb{P}(13,11,10), \text { Diff }=\left(\frac{1}{2}, 0,0,0\right)\end{array}$ & 14 \\
\hline 16 & $x^{5} y+z^{2} y^{4}$ & $\begin{array}{c}t^{2}+z^{5}+x y+z^{2} y^{4} \subset \mathbb{P}(10,4,17,3) \\
\text { Diff }=\left(0,0, \frac{4}{5}, 0\right)\end{array}$ & 10 \\
\hline 17 & $\begin{array}{c}x^{4} y^{2}+y^{n} \\
n=7 ; 9\end{array}$ & $\begin{array}{l}t^{2}+z+x^{2} y^{2}+y^{n} \subset \mathbb{P}(n, 2 n, n-2,2) \\
\quad \mathbb{P}(n, n-2,2), \text { Diff }=\left(0, \frac{4}{5}, \frac{1}{2}, 0\right)\end{array}$ & 15,20 \\
\hline 18 & $x^{4} y^{2}+a x^{2} y^{5}+b y^{8}$ & $\begin{array}{c}t^{2}+z+x^{2} y^{2}+a x y^{5}+b y^{8} \subset \mathbb{P}(4,8,3,1) \\
\mathbb{P}(4,3,1), \text { Diff }=\left(0, \frac{4}{5}, \frac{1}{2}, 0\right)\end{array}$ & 10 \\
\hline 19 & $\begin{array}{c}x^{4} y^{2}+x y^{n} \\
n=6 ; 7\end{array}$ & $\begin{array}{c}t^{2}+z+x^{4} y^{2}+x y^{n} \subset \mathbb{P}(2 n-1,4 n-2, n-2,3) \\
\mathbb{P}(2 n-1, n-2,3), \text { Diff }=\left(0, \frac{4}{5}, 0,0\right)\end{array}$ & 10,15 \\
\hline 20 & $\begin{array}{c}x^{4} y^{2}+z y^{n} \\
n=5 ; 7\end{array}$ & $\begin{array}{c}t^{2}+z^{5}+x^{2} y^{2}+z y^{n} \subset \mathbb{P}(5 n, 2 n, 5 n-8,8) \\
\text { Diff }=\left(0,0, \frac{1}{2}, 0\right)\end{array}$ & 11,16 \\
\hline 21 & $x^{4} y^{2}+z y^{6}$ & $\begin{array}{c}t^{2}+z^{5}+x^{2} y^{2}+z y^{6} \subset \mathbb{P}(15,6,11,4) \\
\text { Diff }=\left(0,0, \frac{1}{2}, 0\right)\end{array}$ & 8 \\
\hline 22 & $\begin{array}{c}x^{4} y^{2}+z x y^{n} \\
n=4 ; 5\end{array}$ & $\begin{array}{c}t^{2}+z^{5}+g(z, x, y) \subset \mathbb{P}(10 n-5,4 n-2,5 n-8,11) \\
\text { Diff }=\varnothing\end{array}$ & 6,11 \\
\hline 23 & $x^{4} y^{2}+a z^{2} y^{4}+b z x^{2} y^{3}$ & $\begin{array}{c}t^{2}+z^{5}+g\left(z, x^{1 / 2}, y\right) \subset \mathbb{P}(10,4,7,3) \\
\text { Diff }=\left(0,0, \frac{1}{2}, 0\right)\end{array}$ & 6 \\
\hline 24 & $x^{4} y^{2}+z^{2} y^{5}$ & $\begin{array}{c}t^{2}+z^{5}+x^{2} y^{2}+z^{2} y^{5} \subset \mathbb{P}(25,10,19,6) \\
\text { Diff }=\left(0,0, \frac{1}{2}, 0\right)\end{array}$ & 12 \\
\hline
\end{tabular}


Продолжение таблицы 5

\begin{tabular}{|c|c|c|c|}
\hline 25 & $x^{4} y^{2}+z^{2} x y^{3}$ & $\begin{array}{c}t^{2}+z^{5}+g(z, x, y) \subset \mathbb{P}(25,10,9,7) \\
\text { Diff }=\varnothing\end{array}$ & 7 \\
\hline 26 & $z x^{5}+y^{7}$ & $\begin{array}{l}t+z^{5}+z x+y \subset \mathbb{P}(5,1,4,5) \\
\mathbb{P}(1,4,5), \text { Diff }=\left(\frac{1}{2}, 0, \frac{4}{5}, \frac{6}{7}\right)\end{array}$ & 50 \\
\hline 27 & $z x^{5}+z^{2} y^{4}$ & $\begin{array}{c}t^{2}+z^{5}+z x+z^{2} y^{2} \subset \mathbb{P}(5,2,8,3) \\
\text { Diff }=\left(0,0, \frac{4}{5}, \frac{1}{2}\right)\end{array}$ & 15 \\
\hline 28 & $z x^{5}+z^{2} x y^{3}$ & $\begin{array}{c}t+z^{5}+z x^{5}+z^{2} x y \subset \mathbb{P}(25,5,4,11) \\
\mathbb{P}(5,4,11), \text { Diff }=\left(\frac{1}{2}, 0,0, \frac{2}{3}\right)\end{array}$ & 22 \\
\hline 29 & $z x^{4} y+y^{7}$ & $\begin{array}{c}t^{2}+z^{5}+z x^{2} y+y^{7} \subset \mathbb{P}(35,14,23,10) \\
\text { Diff }=\left(0,0, \frac{1}{2}, 0\right)\end{array}$ & 20 \\
\hline 30 & $z x^{4} y+x y^{6}$ & $\begin{array}{c}t+z^{5}+z x^{4} y+x y^{6} \subset \mathbb{P}(115,23,19,16) \\
\mathbb{P}(23,19,16), \text { Diff }=\left(\frac{1}{2}, 0,0,0\right)\end{array}$ & 32 \\
\hline 31 & $z x^{4} y+z^{2} y^{4}$ & $\begin{array}{c}t^{2}+z^{5}+z x y+z^{2} y^{4} \subset \mathbb{P}(10,4,13,3) \\
\text { Diff }=\left(0,0, \frac{3}{4}, 0\right)\end{array}$ & 12 \\
\hline 32 & $z x^{4} y+z^{2} x y^{3}$ & $\begin{array}{c}t+z^{5}+z x^{4} y+z^{2} x y^{3} \subset \mathbb{P}(55,11,9,8) \\
\mathbb{P}(11,9,8), \text { Diff }=\left(\frac{1}{2}, 0,0,0\right)\end{array}$ & 16 \\
\hline 33 & $z x^{3} y^{2}+y^{8}$ & $\begin{array}{c}t^{2}+z^{5}+z x y+y^{4} \subset \mathbb{P}(10,4,11,5) \\
\text { Diff }=\left(0,0, \frac{2}{3}, \frac{1}{2}\right)\end{array}$ & 15 \\
\hline 34 & $z x^{3} y^{2}+y^{9}$ & $\begin{array}{c}t+z^{5}+z x y^{2}+y^{9} \subset \mathbb{P}(45,9,26,5) \\
\mathbb{P}(9,26,5), \text { Diff }=\left(\frac{1}{2}, 0, \frac{2}{3}, 0\right)\end{array}$ & 30 \\
\hline 35 & $z x^{3} y^{2}+x y^{6}$ & $\begin{array}{c}t^{2}+z^{5}+z x^{3} y+x y^{3} \subset \mathbb{P}(20,8,7,11) \\
\text { Diff }=\left(0,0,0, \frac{1}{2}\right)\end{array}$ & 11 \\
\hline 36 & $z x^{3} y^{2}+x y^{7}$ & $\begin{array}{c}t+z^{5}+z x^{3} y^{2}+x y^{7} \subset \mathbb{P}(95,19,18,11) \\
\mathbb{P}(19,18,11), \text { Diff }=\left(\frac{1}{2}, 0,0,0\right)\end{array}$ & 22 \\
\hline 37 & $z x^{3} y^{2}+x^{2} y^{5}$ & $\begin{array}{c}t+z^{5}+z x^{3} y^{2}+x^{2} y^{5} \subset \mathbb{P}(55,11,10,7) \\
\mathbb{P}(11,10,7), \text { Diff }=\left(\frac{1}{2}, 0,0,0\right)\end{array}$ & 14 \\
\hline 38 & $z x^{3} y^{2}+z^{2} y^{5}$ & $\begin{array}{c}t+z^{5}+z x y^{2}+z^{2} y^{5} \subset \mathbb{P}(25,5,14,3) \\
\mathbb{P}(5,14,3), \text { Diff }=\left(\frac{1}{2}, 0, \frac{2}{3}, 0\right)\end{array}$ & 18 \\
\hline
\end{tabular}

ТАБлицА 6. Особенность: $t^{2}+z^{4} x+g(z, x, y)$

\begin{tabular}{|c|c|c|c|}
\hline 1 & $x^{i} y^{j} f_{6-i-j}(x, y)$ & $t^{2}+z x+g(z, x, y) \subset \mathbb{P}(3,5,1,1)$ & 4 \\
& $i \leqslant 1 j \leqslant 2$ & Diff $=\left(0, \frac{3}{4}, 0,0\right)$ & \\
\hline 2 & $x^{6}+y^{7}$ & $t^{2}+z x+x^{6}+y \subset \mathbb{P}(3,5,1,6)$ & 8 \\
& & $\mathbb{P}(3,5,1), \operatorname{Diff}=\left(0, \frac{3}{4}, 0, \frac{6}{7}\right)$ & \\
\hline 3 & $x^{6}+x^{2} y^{5}$ & $t^{2}+z x+x^{6}+x^{2} y \subset \mathbb{P}(3,5,1,4)$ & 8 \\
& & $\operatorname{Diff}=\left(0, \frac{3}{4}, 0, \frac{4}{5}\right)$ & \\
\hline 4 & $x^{6}+z y^{n}$ & $t^{2}+z^{4} x+x^{6}+z y \subset \mathbb{P}(12,5,4,19)$ & 5,19 \\
& $n=5 ; 6$ & Diff $=\left(0,0,0, \frac{n-1}{n}\right)$ & \\
\hline
\end{tabular}


Продолжение таблицы 6

\begin{tabular}{|c|c|c|c|}
\hline 5 & $a x^{6}+b z x^{3} y^{2}+z^{2} y^{4}$ & $\begin{array}{c}t^{2}+z^{4} x+a x^{6}+b z x^{3} y+z^{2} y^{2} \subset \mathbb{P}(12,5,4,7) \\
\text { Diff }=\left(0,0,0, \frac{1}{2}\right)\end{array}$ & 7 \\
\hline 6 & $x^{5} y+y^{7}$ & $\begin{array}{c}t^{2}+z^{2} x+x^{5} y+y^{7} \subset \mathbb{P}(35,29,12,10) \\
\text { Diff }=\left(0, \frac{1}{2}, 0,0\right)\end{array}$ & 8 \\
\hline 7 & $x^{5} y+y^{8}$ & $\begin{array}{c}t^{2}+z x+x^{5} y+y^{8} \subset \mathbb{P}(20,33,7,5) \\
\text { Diff }=\left(0, \frac{3}{4}, 0,0\right)\end{array}$ & 20 \\
\hline 8 & $x^{5} y+x^{2} y^{5}$ & $\begin{array}{c}t^{2}+z^{2} x+x^{5} y+x^{2} y^{5} \subset \mathbb{P}(23,19,8,6) \\
\text { Diff }=\left(0, \frac{1}{2}, 0,0\right)\end{array}$ & 12 \\
\hline 9 & $\begin{array}{c}x^{5} y+z y^{n} \\
n=5 ; 6\end{array}$ & $\begin{array}{c}t+z^{4} x+x^{5} y+z y^{n} \subset \mathbb{P}(20 n+1,4 n+1,4 n-3,16) \\
\mathbb{P}(4 n+1,4 n-3,16), \text { Diff }=\left(\frac{1}{2}, 0,0,0\right)\end{array}$ & 6,14 \\
\hline 10 & $x^{5} y+z^{2} y^{4}$ & $\begin{array}{c}t^{2}+z^{4} x+x^{5} y+z^{2} y^{4} \subset \mathbb{P}(41,17,14,12) \\
\text { Diff }=\varnothing\end{array}$ & 6 \\
\hline 11 & $x^{5} y+z^{3} y^{3}$ & $\begin{array}{c}t+z^{4} x+x^{5} y+z^{3} y^{3} \subset \mathbb{P}(63,13,11,8) \\
\mathbb{P}(13,11,8), \text { Diff }=\left(\frac{1}{2}, 0,0,0\right)\end{array}$ & 16 \\
\hline 12 & $\begin{array}{c}x^{4} y^{2}+y^{n} \\
n=7 ; 9\end{array}$ & $\begin{array}{c}t^{2}+z x+x^{4} y^{2}+y^{n} \subset \mathbb{P}(2 n, 3 n+2, n-2,4) \\
\text { Diff }=\left(0, \frac{3}{4}, 0,0\right)\end{array}$ & 12,16 \\
\hline 13 & $x^{4} y^{2}+y^{8}$ & $\begin{array}{c}t^{2}+z x+x^{4} y^{2}+y^{8} \subset \mathbb{P}(8,13,3,2) \\
\text { Diff }=\left(0, \frac{3}{4}, 0,0\right)\end{array}$ & 8 \\
\hline 14 & $\begin{array}{c}x^{4} y^{2}+z y^{n} \\
n=5,6 ; 7\end{array}$ & $\begin{aligned} t^{2}+z^{4} x+g(z, x, y) & \subset \mathbb{P}(8 n+1,3 n+2,4 n-6,13) \\
& \text { Diff }=\varnothing\end{aligned}$ & 9,13 \\
\hline 15 & $x^{4} y^{2}+a z x^{2} y^{3}+z^{2} y^{4}$ & $\begin{array}{c}t^{2}+z^{4} x+g(z, x, y) \subset \mathbb{P}(17,7,6,5) \\
\text { Diff }=\varnothing\end{array}$ & 5 \\
\hline 16 & $x^{4} y^{2}+z^{2} y^{5}$ & $\begin{array}{c}t^{2}+z^{2} x+x^{4} y^{2}+z y^{5} \subset \mathbb{P}(21,17,8,5) \\
\text { Diff }=\left(0, \frac{1}{2}, 0,0\right)\end{array}$ & 10 \\
\hline 17 & $x^{4} y^{2}+z^{3} y^{3}$ & $\begin{array}{c}t^{2}+z^{4} x+g(z, x, y) \subset \mathbb{P}(27,11,10,7) \\
\text { Diff }=\varnothing\end{array}$ & 7 \\
\hline 18 & $z x^{5}+y^{7}$ & $\begin{array}{c}t+z^{4} x+z x^{5}+y \subset \mathbb{P}(19,4,3,19) \\
\mathbb{P}(4,3,19), \text { Diff }=\left(\frac{1}{2}, 0,0, \frac{6}{7}\right)\end{array}$ & 14 \\
\hline 19 & $z x^{5}+x^{2} y^{5}$ & $\begin{array}{c}t+z^{4} x+z x^{5}+x^{2} y \subset \mathbb{P}(19,4,3,13) \\
\mathbb{P}(4,3,13), \text { Diff }=\left(\frac{1}{2}, 0,0, \frac{4}{5}\right)\end{array}$ & 26 \\
\hline 20 & $z x^{5}+z^{2} y^{4}$ & $\begin{array}{c}t^{2}+z^{4} x+z x^{5}+z^{2} y^{2} \subset \mathbb{P}(19,8,6,11) \\
\text { Diff }=\left(0,0,0, \frac{1}{2}\right)\end{array}$ & 11 \\
\hline 21 & $z x^{4} y+y^{7}$ & $\begin{array}{c}t+z^{4} x+z x^{4} y+y^{7} \subset \mathbb{P}(105,22,17,15) \\
\mathbb{P}(22,17,15), \text { Diff }=\left(\frac{1}{2}, 0,0,0\right)\end{array}$ & 10 \\
\hline 22 & $z x^{4} y+x^{2} y^{5}$ & $\begin{array}{c}t+z^{4} x+z x^{4} y+x^{2} y^{5} \subset \mathbb{P}(67,14,11,9) \\
\mathbb{P}(14,11,9), \text { Diff }=\left(\frac{1}{2}, 0,0,0\right)\end{array}$ & 18 \\
\hline 23 & $z x^{4} y+z^{2} y^{4}$ & $\begin{array}{c}t^{2}+z^{4} x+g(z, x, y) \subset \mathbb{P}(31,13,10,9) \\
\text { Diff }=\varnothing\end{array}$ & 9 \\
\hline
\end{tabular}


Продолжение таблицы 6

\begin{tabular}{|c|c|c|c|}
\hline 24 & $z x^{3} y^{2}+y^{n}$ & $t+z^{4} x+z x^{3} y^{2}+y^{n} \subset \mathbb{P}(11 n, 2 n+2,3 n-8,11)$ & 16,22 \\
& $n=7 ; 9$ & $\mathbb{P}(2 n+2,3 n-8,11), \mathrm{Diff}=\left(\frac{1}{2}, 0,0,0\right)$ & \\
\hline 25 & $z x^{3} y^{2}+y^{8}$ & $t^{2}+z^{4} x+z x^{3} y+y^{4} \subset \mathbb{P}(22,9,8,11)$ & 11 \\
& & $\operatorname{Diff}=\left(0,0,0, \frac{1}{2}\right)$ & \\
\hline 26 & $z x^{3} y^{2}+x^{2} y^{5}$ & $t+z^{4} x+z x^{3} y^{2}+x^{2} y^{5} \subset \mathbb{P}(39,8,7,5)$ & 10 \\
& & $\mathbb{P}(8,7,5), \mathrm{Diff}=\left(\frac{1}{2}, 0,0,0\right)$ & \\
\hline 27 & $z x^{3} y^{2}+z^{2} y^{5}$ & $t+z^{4} x+z x^{3} y^{2}+z^{2} y^{5} \subset \mathbb{P}(59,12,11,7)$ & 14 \\
& & $\mathbb{P}(12,11,7), \mathrm{Diff}=\left(\frac{1}{2}, 0,0,0\right)$ & \\
\hline
\end{tabular}

ТАБЛИЦА 7 . Особенность: $t^{2}+z^{4} y+g(z, x, y)$

\begin{tabular}{|c|c|c|c|}
\hline 1 & $\begin{array}{l}x^{6}+a z x^{3} y^{2}+b z^{2} y^{4}+ \\
\quad+c y^{7},|b|+|c| \neq 0\end{array}$ & $\begin{array}{c}t^{2}+z^{4} y+g\left(z, x^{1 / 3}, y\right) \subset \mathbb{P}(7,3,7,2) \\
\text { Diff }=\left(0,0, \frac{2}{3}, 0\right)\end{array}$ & 6 \\
\hline 2 & $x^{6}+a x^{3} y^{4}+b y^{8}$ & $\begin{array}{c}t^{2}+z y+x^{2}+a x y^{4}+b y^{8} \subset \mathbb{P}(4,7,4,1) \\
\text { Diff }=\left(0, \frac{3}{4}, \frac{2}{3}, 0\right)\end{array}$ & 12 \\
\hline 3 & $\begin{array}{c}x^{6}+x y^{n} \\
n=6 ; 7\end{array}$ & $\begin{array}{c}t^{2}+z y+x^{6}+x y^{n} \subset \mathbb{P}(3 n, 6 n-5, n, 5) \\
\text { Diff }=\left(0, \frac{3}{4}, 0,0\right)\end{array}$ & 8,20 \\
\hline 4 & $x^{6}+a z x y^{3}+b x^{2} y^{5}$ & $\begin{array}{c}t^{2}+z^{2} y+g(z, x, y) \subset \mathbb{P}(15,13,5,4) \\
\text { Diff }=\left(0, \frac{1}{2}, 0,0\right)\end{array}$ & 8 \\
\hline 5 & $\begin{array}{c}x^{6}+z y^{n} \\
n=5 ; 6\end{array}$ & $\begin{array}{c}t^{2}+z^{4} y+x^{2}+z y^{n} \subset \mathbb{P}(4 n-1,2 n-2,4 n-1,6) \\
\text { Diff }=\left(0,0, \frac{2}{3}, 0\right)\end{array}$ & 6,9 \\
\hline 6 & $\begin{array}{c}x^{6}+z x y^{n} \\
n=4 ; 5\end{array}$ & $\begin{array}{c}t^{2}+z^{4} y+g(z, x, y) \subset \mathbb{P}(12 n-3,6 n-5,4 n-1,14) \\
\text { Diff }=\varnothing\end{array}$ & 5,14 \\
\hline 7 & $x^{6}+z x^{2} y^{3}$ & $\begin{array}{c}t^{2}+z^{4} y+g(z, x, y) \subset \mathbb{P}(33,14,11,10) \\
\text { Diff }=\varnothing\end{array}$ & 5 \\
\hline 8 & $\begin{array}{c}a z^{3} x^{2}+b z^{2} x y^{3}+c z x^{3} y^{2}+ \\
d z y^{6}+e x^{5} y+k x^{2} y^{5}+l z^{4} y \\
\text { канонич. } \&|e|+|c| \neq 0\end{array}$ & $\begin{array}{c}t+z^{4} y+g(z, x, y) \subset \mathbb{P}(23,5,4,3) \\
\mathbb{P}(5,4,3), \text { Diff }=\left(\frac{1}{2}, 0,0,0\right)\end{array}$ & 6 \\
\hline 9 & $z x^{5}+a z^{2} y^{4}+b y^{7}$ & $\begin{array}{c}t^{2}+z^{4} y+z x+a z^{2} y^{4}+b y^{7} \subset \mathbb{P}(7,3,11,2) \\
\text { Diff }=\left(0,0, \frac{4}{5}, 0\right)\end{array}$ & 10 \\
\hline 10 & $z x^{5}+x y^{6}$ & $\begin{array}{c}t+z^{4} y+z x^{5}+x y^{6} \subset \mathbb{P}(121,26,19,17) \\
\mathbb{P}(26,19,17), \text { Diff }=\left(\frac{1}{2}, 0,0,0\right)\end{array}$ & 24 \\
\hline 11 & $z x^{5}+x^{2} y^{5}$ & $\begin{array}{c}t+z^{4} y+z x^{5}+x^{2} y^{5} \subset \mathbb{P}(51,11,8,7) \\
\mathbb{P}(11,8,7), \text { Diff }=\left(\frac{1}{2}, 0,0,0\right)\end{array}$ & 16 \\
\hline 12 & $z x^{5}+x^{3} y^{4}$ & $\begin{array}{c}t+z^{4} y+z x^{5}+x^{3} y^{4} \subset \mathbb{P}(83,18,13,11) \\
\mathbb{P}(18,13,11), \text { Diff }=\left(\frac{1}{2}, 0,0,0\right)\end{array}$ & 22 \\
\hline
\end{tabular}

ТАБлицА 8. Особенность: $t^{2}+z^{3} x^{2}+g(z, x, y)$

\begin{tabular}{|c|c|c|c|}
\hline 1 & $x^{i} y^{j} f_{6-i-j}(x, y)$ & $t^{2}+z x^{2}+g(z, x, y) \subset \mathbb{P}(3,4,1,1)$ & 3 \\
& $i \leqslant 1 j \leqslant 3$ & $\operatorname{Diff}=\left(0, \frac{2}{3}, 0,0\right)$ & \\
\hline
\end{tabular}


Продолжение таблицы 8

\begin{tabular}{|c|c|c|c|}
\hline 2 & $x^{6}+y^{7}$ & $\begin{array}{c}t^{2}+z x^{2}+x^{6}+y \subset \mathbb{P}(3,4,1,6) \\
\mathbb{P}(3,4,1), \text { Diff }=\left(0, \frac{2}{3}, 0, \frac{6}{7}\right)\end{array}$ & 7 \\
\hline 3 & $a x^{6}+b x^{3} y^{4}+y^{8}$ & $\begin{array}{c}t^{2}+z x^{2}+a x^{6}+b x^{3} y+y^{2} \subset \mathbb{P}(3,4,1,3) \\
\text { Diff }=\left(0, \frac{2}{3}, 0, \frac{3}{4}\right)\end{array}$ & 9 \\
\hline 4 & $\begin{array}{c}z^{2} y^{4}+b z x^{3} y^{2}+c x^{6}+d x y^{6} \\
|b|+|c| \neq 0\end{array}$ & $\begin{array}{c}t^{2}+z^{3} x^{2}+g\left(z, x, y^{1 / 2}\right) \subset \mathbb{P}(9,4,3,5) \\
\text { Diff }=\left(0,0,0, \frac{1}{2}\right)\end{array}$ & 5 \\
\hline 5 & $x^{6}+x y^{7}$ & $\begin{array}{c}t^{2}+z x^{2}+x^{6}+x y \subset \mathbb{P}(3,4,1,5) \\
\text { Diff }=\left(0, \frac{2}{3}, 0, \frac{6}{7}\right)\end{array}$ & 15 \\
\hline 6 & $x^{6}+z y^{5}$ & $\begin{array}{c}t^{2}+z^{3} x^{2}+x^{6}+z y \subset \mathbb{P}(9,4,3,14) \\
\operatorname{Diff}=\left(0,0,0, \frac{4}{5}\right)\end{array}$ & 5 \\
\hline 7 & $x^{6}+z y^{6}$ & $\begin{array}{c}t^{2}+z^{3} x^{2}+x^{6}+z y^{2} \subset \mathbb{P}(9,4,3,7) \\
\text { Diff }=\left(0,0,0, \frac{2}{3}\right)\end{array}$ & 7 \\
\hline 8 & $\begin{array}{c}x^{6}+z x y^{n} \\
n=4 ; 5\end{array}$ & $\begin{array}{c}t^{2}+z^{3} x^{2}+x^{6}+z x y \subset \mathbb{P}(9,4,3,11) \\
\text { Diff }=\left(0,0,0, \frac{n-1}{n}\right)\end{array}$ & 4,11 \\
\hline 9 & $\begin{array}{c}x^{5} y+y^{n} \\
n=7 ; 9\end{array}$ & $\begin{array}{l}t+z x^{2}+x^{5} y+y^{n} \subset \mathbb{P}(5 n, 3 n+2, n-1,5) \\
\quad \mathbb{P}(3 n+2, n-1,5), \text { Diff }=\left(\frac{1}{2}, \frac{2}{3}, 0,0\right)\end{array}$ & 15,30 \\
\hline 10 & $x^{5} y+y^{8}$ & $\begin{array}{c}t^{2}+z x^{2}+x^{5} y+y^{8} \subset \mathbb{P}(20,26,7,5) \\
\text { Diff }=\left(0, \frac{2}{3}, 0,0\right)\end{array}$ & 13 \\
\hline 11 & $x^{5} y+x y^{6}$ & $\begin{array}{l}t+z x^{2}+x^{5} y+x y^{6} \subset \mathbb{P}(29,19,5,4) \\
\mathbb{P}(19,5,4), \text { Diff }=\left(\frac{1}{2}, \frac{2}{3}, 0,0\right)\end{array}$ & 6 \\
\hline 12 & $\begin{array}{c}a z^{3} y^{3}+b x^{5} y+c x^{3} y^{4}+d x y^{7} \\
|b|+|c| \neq 0,|a|+|d| \neq 0\end{array}$ & $\begin{array}{c}t+z x^{2}+g(z, x, y) \subset \mathbb{P}(17,11,3,2) \\
\mathbb{P}(11,3,2), \text { Diff }=\left(\frac{1}{2}, \frac{2}{3}, 0,0\right)\end{array}$ & 12 \\
\hline 13 & $\begin{array}{c}x^{5} y+z y^{n} \\
n=5 ; 7\end{array}$ & $\begin{array}{c}t+z^{3} x^{2}+x^{5} y+z y^{n} \subset \mathbb{P}(15 n+2,3 n+2,3 n-2,12) \\
\mathbb{P}(3 n+2,3 n-2,12), \text { Diff }=\left(\frac{1}{2}, 0,0,0\right)\end{array}$ & 11,24 \\
\hline 14 & $x^{5} y+z x y^{4}$ & $\begin{array}{c}t+z^{3} x^{2}+x^{5} y+z x y^{4} \subset \mathbb{P}(59,13,10,9) \\
\mathbb{P}(13,10,9), \text { Diff }=\left(\frac{1}{2}, 0,0,0\right)\end{array}$ & 4 \\
\hline 15 & $x^{5} y+z x y^{5}$ & $\begin{array}{c}t^{2}+z^{3} x^{2}+x^{5} y+z x y^{5} \subset \mathbb{P}(37,16,13,9) \\
\text { Diff }=\varnothing\end{array}$ & 9 \\
\hline 16 & $x^{5} y+z^{2} y^{4}$ & $\begin{array}{c}t^{2}+z^{3} x^{2}+x^{5} y+z^{2} y^{4} \subset \mathbb{P}(32,14,11,9) \\
\text { Diff }=\varnothing\end{array}$ & 7 \\
\hline 17 & $x^{5} y+z^{2} y^{5}$ & $\begin{array}{c}t+z^{3} x^{2}+x^{5} y+z^{2} y^{5} \subset \mathbb{P}(79,17,14,9) \\
\mathbb{P}(17,14,9), \text { Diff }=\left(\frac{1}{2}, 0,0,0\right)\end{array}$ & 18 \\
\hline 18 & $\begin{array}{c}a z^{4} y+b z^{2} y^{4}+c z x^{2} y^{3}+ \\
+x^{4} y^{2}+d y^{7}\end{array}$ & $\begin{array}{c}t^{2}+z^{3} x^{2}+g\left(z, x^{1 / 2}, y\right) \subset \mathbb{P}(7,3,5,2) \\
\text { Diff }=\left(0,0, \frac{1}{2}, 0\right)\end{array}$ & 4 \\
\hline 19 & $\begin{array}{c}a z^{3} y^{3}+x^{4} y^{2}+b x^{2} y^{5}+ \\
+c y^{8},|a|+|c| \neq 0\end{array}$ & $\begin{array}{c}t^{2}+z x+g\left(z^{1 / 3}, x^{1 / 2}, y\right) \subset \mathbb{P}(4,5,3,1) \\
\text { Diff }=\left(0, \frac{2}{3}, \frac{1}{2}, 0\right)\end{array}$ & 6 \\
\hline 20 & $x^{4} y^{2}+y^{9}$ & $\begin{array}{c}t^{2}+z x+x^{2} y^{2}+y^{9} \subset \mathbb{P}(9,11,7,2) \\
\text { Diff }=\left(0, \frac{2}{3}, \frac{1}{2}, 0\right)\end{array}$ & 12 \\
\hline
\end{tabular}


Продолжение таблицы 8

\begin{tabular}{|c|c|c|c|}
\hline 21 & $\begin{array}{c}x^{4} y^{2}+x y^{n} \\
n=6 ; 7\end{array}$ & $\begin{array}{c}t^{2}+z x^{2}+g(z, x, y) \subset \mathbb{P}(2 n-1,2 n+2, n-2,3) \\
\text { Diff }=\left(0, \frac{2}{3}, 0,0\right)\end{array}$ & 6,9 \\
\hline 22 & $\begin{array}{c}x^{4} y^{2}+z y^{n} \\
n=5 ; 7\end{array}$ & $\begin{array}{c}t^{2}+z^{3} x+x^{2} y^{2}+z y^{n} \subset \mathbb{P}(3 n+1, n+2,3 n-4,5) \\
\text { Diff }=\left(0,0, \frac{1}{2}, 0\right)\end{array}$ & 7,10 \\
\hline 23 & $a z^{2} x y^{3}+b z y^{6}+x^{4} y^{2}$ & $\begin{array}{c}t^{2}+z^{3} x^{2}+g(z, x, y) \subset \mathbb{P}(19,8,7,5) \\
\text { Diff }=\varnothing\end{array}$ & 5 \\
\hline 24 & $\begin{array}{c}x^{4} y^{2}+z x y^{n} \\
n=4 ; 5\end{array}$ & $\begin{array}{c}t^{2}+z^{3} x^{2}+g(z, x, y) \subset \mathbb{P}(6 n-1,2 n+2,3 n-4,7) \\
\text { Diff }=\varnothing\end{array}$ & 4,7 \\
\hline 25 & $x^{4} y^{2}+z^{2} y^{5}$ & $\begin{array}{c}t^{2}+z^{3} x+x^{2} y^{2}+z^{2} y^{5} \subset \mathbb{P}(17,7,13,4) \\
\text { Diff }=\left(0,0, \frac{1}{2}, 0\right)\end{array}$ & 8 \\
\hline 26 & $\begin{array}{c}x^{3} y^{3}+y^{n} \\
n=7 ; 11\end{array}$ & $\begin{array}{c}t+z x^{2}+x^{3} y^{3}+y^{n} \subset \mathbb{P}(3 n, n+6, n-3,3) \\
\mathbb{P}(n+6, n-3,3), \text { Diff }=\left(\frac{1}{2}, \frac{2}{3}, 0,0\right)\end{array}$ & 10,18 \\
\hline 27 & $\begin{array}{c}x^{3} y^{3}+y^{2 n} \\
n=4 ; 5\end{array}$ & $\begin{array}{c}t^{2}+z x^{2}+x^{3} y^{3}+y^{2 n} \subset \mathbb{P}(3 n, 2 n+6,2 n-3,3) \\
\text { Diff }=\left(0, \frac{2}{3}, 0,0\right)\end{array}$ & 7,9 \\
\hline 28 & $a z^{3} y^{5}+x^{3} y^{3}+b x y^{8}$ & $\begin{array}{c}t+z x^{2}+g(z, x, y) \subset \mathbb{P}(21,11,5,2) \\
\mathbb{P}(11,5,2), \text { Diff }=\left(\frac{1}{2}, \frac{2}{3}, 0,0\right)\end{array}$ & 12 \\
\hline 29 & $\begin{array}{c}x^{3} y^{3}+z y^{n} \\
n=5 ; 7\end{array}$ & $\begin{array}{c}t+z^{3} x^{2}+x^{3} y^{3}+z y^{n} \subset \mathbb{P}(9 n+6, n+6,3 n-6,8) \\
\mathbb{P}(n+6,3 n-6,8), \text { Diff }=\left(\frac{1}{2}, 0,0,0\right)\end{array}$ & 8,10 \\
\hline 30 & $a z^{5} y+b z^{2} x y^{4}+c z y^{8}+x^{3} y^{3}$ & $\begin{array}{c}t+z^{3} x^{2}+g(z, x, y) \subset \mathbb{P}(39,7,9,4) \\
\mathbb{P}(7,9,4), \text { Diff }=\left(\frac{1}{2}, 0,0,0\right)\end{array}$ & 8 \\
\hline 31 & $x^{3} y^{3}+z y^{9}$ & $\begin{array}{c}t+z^{3} x^{2}+x^{3} y+z y^{3} \subset \mathbb{P}(29,5,7,8) \\
\mathbb{P}(5,7,8), \text { Diff }=\left(\frac{1}{2}, 0,0, \frac{2}{3}\right)\end{array}$ & 16 \\
\hline 32 & $a z^{4} y+b z x y^{4}+x^{3} y^{3}$ & $\begin{array}{c}t+z^{3} x^{2}+g(z, x, y) \subset \mathbb{P}(33,7,6,5) \\
\mathbb{P}(7,6,5), \text { Diff }=\left(\frac{1}{2}, 0,0,0\right)\end{array}$ & 4 \\
\hline 33 & $a z^{4} y^{2}+b z x y^{5}+x^{3} y^{3}$ & $\begin{array}{c}t^{2}+z^{3} x^{2}+g(z, x, y) \subset \mathbb{P}(21,8,9,5) \\
\text { Diff }=\varnothing\end{array}$ & 5 \\
\hline 34 & $a z^{4} y^{3}+b z x y^{6}+x^{3} y^{3}$ & $\begin{array}{c}t+z^{3} x^{2}+g\left(z, x, y^{1 / 3}\right) \subset \mathbb{P}(17,3,4,5) \\
\mathbb{P}(3,4,5), \text { Diff }=\left(\frac{1}{2}, 0,0, \frac{2}{3}\right)\end{array}$ & 10 \\
\hline 35 & $z^{2} y^{4}+x^{3} y^{3}$ & $\begin{array}{c}t^{2}+z^{3} x^{2}+g(z, x, y) \subset \mathbb{P}(24,10,9,7) \\
\text { Diff }=\varnothing\end{array}$ & 5 \\
\hline 36 & $\begin{array}{c}x^{3} y^{3}+z^{2} y^{n} \\
n=5 ; 7\end{array}$ & $\begin{array}{c}t+z^{3} x^{2}+g(z, x, y) \subset \mathbb{P}(9 n+12, n+6,3 n-3,7) \\
\mathbb{P}(n+6,3 n-3,7), \text { Diff }=\left(\frac{1}{2}, 0,0,0\right)\end{array}$ & 8,14 \\
\hline 37 & $x^{3} y^{3}+z^{2} y^{6}$ & $\begin{array}{c}t^{2}+z^{3} x^{2}+x^{3} y+z^{2} y^{2} \subset \mathbb{P}(11,4,5,7) \\
\text { Diff }=\left(0,0,0, \frac{2}{3}\right)\end{array}$ & 7 \\
\hline 38 & $\begin{array}{c}x^{i} y^{j} f_{7-i-j}(x, y) \\
i \leqslant 1 j \leqslant 4\end{array}$ & $\begin{array}{c}t+z x^{2}+g(z, x, y) \subset \mathbb{P}(7,5,1,1) \\
\text { Diff }=\left(\frac{1}{2}, \frac{2}{3}, 0,0\right)\end{array}$ & 6 \\
\hline 39 & $x^{7}+y^{8}$ & $\begin{array}{c}t^{2}+z x^{2}+x^{7}+y^{2} \subset \mathbb{P}(7,10,2,7) \\
\text { Diff }=\left(0, \frac{2}{3}, 0, \frac{3}{4}\right)\end{array}$ & 12 \\
\hline
\end{tabular}


Продолжение таблицы 8

\begin{tabular}{|c|c|c|c|}
\hline 40 & $x^{7}+x y^{7}$ & $\begin{array}{c}t+z x^{2}+x^{7}+x y \subset \mathbb{P}(7,5,1,6) \\
\mathbb{P}(5,1,6), \text { Diff }=\left(\frac{1}{2}, \frac{2}{3}, 0, \frac{6}{7}\right)\end{array}$ & 36 \\
\hline 41 & $a z^{2} x y^{3}+b z y^{6}+x^{7}$ & $\begin{array}{c}t+z^{3} x^{2}+g\left(z, x, y^{1 / 3}\right) \subset \mathbb{P}(21,5,3,8) \\
\mathbb{P}(5,3,8), \text { Diff }=\left(\frac{1}{2}, 0,0, \frac{2}{3}\right)\end{array}$ & 16 \\
\hline 42 & $x^{7}+z x y^{5}$ & $\begin{array}{c}t+z^{3} x^{2}+x^{7}+z x y \subset \mathbb{P}(21,5,3,13) \\
\mathbb{P}(5,3,13), \text { Diff }=\left(\frac{1}{2}, 0,0, \frac{4}{5}\right)\end{array}$ & 26 \\
\hline 43 & $x^{7}+z^{2} y^{4}$ & $\begin{array}{c}t^{2}+z^{3} x^{2}+x^{7}+z^{2} y^{2} \subset \mathbb{P}(21,10,6,11) \\
\text { Diff }=\left(0,0,0, \frac{1}{2}\right)\end{array}$ & 11 \\
\hline 44 & $x^{6} y+y^{8}$ & $\begin{array}{c}t^{2}+z x+x^{3} y+y^{8} \subset \mathbb{P}(12,17,7,3) \\
\text { Diff }=\left(0, \frac{2}{3}, \frac{1}{2}, 0\right)\end{array}$ & 18 \\
\hline 45 & $x^{6} y+x y^{7}$ & $\begin{array}{c}t+z x^{2}+x^{6} y+x y^{7} \subset \mathbb{P}(41,29,6,5) \\
\mathbb{P}(29,6,5), \text { Diff }=\left(\frac{1}{2}, \frac{2}{3}, 0,0\right)\end{array}$ & 30 \\
\hline 46 & $a z^{2} x y^{3}+b z y^{6}+x^{6} y$ & $\begin{array}{c}t+z^{3} x^{2}+g(z, x, y) \subset \mathbb{P}(55,13,8,7) \\
\mathbb{P}(13,8,7), \text { Diff }=\left(\frac{1}{2}, 0,0,0\right)\end{array}$ & 14 \\
\hline 47 & $x^{6} y+z x y^{5}$ & $\begin{array}{c}t+z^{3} x^{2}+x^{6} y+z x y^{5} \subset \mathbb{P}(89,21,13,11) \\
\mathbb{P}(21,13,11), \text { Diff }=\left(\frac{1}{2}, 0,0,0\right)\end{array}$ & 22 \\
\hline 48 & $x^{6} y+z^{2} y^{4}$ & $\begin{array}{c}t^{2}+z^{3} x+x^{3} y+z^{2} y^{4} \subset \mathbb{P}(19,9,11,5) \\
\text { Diff }=\left(0,0, \frac{1}{2}, 0\right)\end{array}$ & 10 \\
\hline 49 & $x^{5} y^{2}+y^{8}$ & $\begin{array}{c}t^{2}+z x^{2}+x^{5} y+y^{4} \subset \mathbb{P}(10,14,3,5) \\
\text { Diff }=\left(0, \frac{2}{3}, 0, \frac{1}{2}\right)\end{array}$ & 15 \\
\hline 50 & $x^{5} y^{2}+x y^{7}$ & $\begin{array}{c}t+z x^{2}+x^{5} y^{2}+x y^{7} \subset \mathbb{P}(33,23,5,4) \\
\mathbb{P}(23,5,4), \text { Diff }=\left(\frac{1}{2}, \frac{2}{3}, 0,0\right)\end{array}$ & 24 \\
\hline 51 & $x^{5} y^{2}+a z^{2} x y^{3}+b z y^{6}$ & $\begin{array}{c}t+z^{3} x^{2}+g(z, x, y) \subset \mathbb{P}(47,11,7,6) \\
\mathbb{P}(11,7,6), \text { Diff }=\left(\frac{1}{2}, 0,0,0\right)\end{array}$ & 12 \\
\hline 52 & $x^{5} y^{2}+z x y^{5}$ & $\begin{array}{c}t+z^{3} x^{2}+x^{5} y^{2}+z x y^{5} \subset \mathbb{P}(73,17,11,9) \\
\mathbb{P}(17,11,9), \text { Diff }=\left(\frac{1}{2}, 0,0,0\right)\end{array}$ & 18 \\
\hline 53 & $x^{5} y^{2}+z^{2} y^{4}$ & $\begin{array}{c}t^{2}+z^{3} x^{2}+x^{5} y+z^{2} y^{2} \subset \mathbb{P}(17,8,5,9) \\
\text { Diff }=\left(0,0,0, \frac{1}{2}\right)\end{array}$ & 9 \\
\hline 54 & $x^{4} y^{3}+y^{8}$ & $\begin{array}{c}t^{2}+z x+x^{2} y^{3}+y^{8} \subset \mathbb{P}(8,11,5,2) \\
\text { Diff }=\left(0, \frac{2}{3}, \frac{1}{2}, 0\right)\end{array}$ & 12 \\
\hline 55 & $x^{4} y^{3}+x y^{7}$ & $\begin{array}{c}t+z x^{2}+x^{4} y^{3}+x y^{7} \subset \mathbb{P}(25,17,4,3) \\
\mathbb{P}(17,4,3), \text { Diff }=\left(\frac{1}{2}, \frac{2}{3}, 0,0\right)\end{array}$ & 18 \\
\hline 56 & $\begin{array}{c}a z^{2} x y^{3}+b z x^{5}+c z y^{6}+d x^{4} y^{3} \\
|b|+|d| \neq 0,|a|+|c| \neq 0\end{array}$ & $\begin{array}{c}t+z^{3} x^{2}+g\left(z, x, y^{1 / 3}\right) \subset \mathbb{P}(13,3,2,5) \\
\mathbb{P}(3,2,5), \text { Diff }=\left(\frac{1}{2}, 0,0, \frac{2}{3}\right)\end{array}$ & 10 \\
\hline 57 & $x^{4} y^{3}+z x y^{5}$ & $\begin{array}{c}t+z^{3} x^{2}+g(z, x, y) \subset \mathbb{P}(57,13,9,7) \\
\mathbb{P}(13,9,7), \text { Diff }=\left(\frac{1}{2}, 0,0,0\right)\end{array}$ & 14 \\
\hline 58 & $x^{4} y^{3}+z^{2} y^{4}$ & $\begin{array}{c}t^{2}+z^{3} x^{2}+x^{2} y^{3}+z^{2} y^{4} \subset \mathbb{P}(15,7,9,4) \\
\text { Diff }=\left(0,0, \frac{1}{2}, 0\right)\end{array}$ & 8 \\
\hline
\end{tabular}


Продолжение таблицы 8

\begin{tabular}{|c|c|c|c|}
\hline 59 & $x^{3} y^{4}+y^{9}$ & $\begin{array}{c}t+z x^{2}+x^{3} y^{4}+y^{9} \subset \mathbb{P}(27,17,5,3) \\
\mathbb{P}(17,5,3), \text { Diff }=\left(\frac{1}{2}, \frac{2}{3}, 0,0\right)\end{array}$ & 18 \\
\hline 60 & $x^{3} y^{4}+z y^{7}$ & $\begin{array}{c}t+z^{3} x^{2}+x^{3} y^{4}+z y^{7} \subset \mathbb{P}(71,15,13,8) \\
\mathbb{P}(15,13,8), \text { Diff }=\left(\frac{1}{2}, 0,0,0\right)\end{array}$ & 16 \\
\hline 61 & $a z^{4} y+b z x y^{5}+x^{3} y^{4}$ & $\begin{array}{c}t+z^{3} x^{2}+g(z, x, y) \subset \mathbb{P}(41,9,7,5) \\
\mathbb{P}(9,7,5), \text { Diff }=\left(\frac{1}{2}, 0,0,0\right)\end{array}$ & 10 \\
\hline 62 & $x^{3} y^{4}+z^{2} y^{5}$ & $\begin{array}{c}t+z^{3} x^{2}+x^{3} y^{4}+z^{2} y^{5} \subset \mathbb{P}(61,13,11,7) \\
\mathbb{P}(13,11,7), \text { Diff }=\left(\frac{1}{2}, 0,0,0\right)\end{array}$ & 14 \\
\hline 63 & $z x^{5}+y^{7}$ & $\begin{array}{c}t+z^{3} x^{2}+z x^{5}+y \subset \mathbb{P}(13,3,2,13) \\
\mathbb{P}(3,2,13), \text { Diff }=\left(\frac{1}{2}, 0,0, \frac{6}{7}\right)\end{array}$ & 14 \\
\hline 64 & $z x^{5}+y^{8}$ & $\begin{array}{c}t^{2}+z^{3} x^{2}+z x^{5}+y^{2} \subset \mathbb{P}(13,6,4,13) \\
\text { Diff }=\left(0,0,0, \frac{3}{4}\right)\end{array}$ & 13 \\
\hline 65 & $z x^{5}+x y^{6}$ & $\begin{array}{c}t^{2}+z^{3} x^{2}+z x^{5}+x y^{2} \subset \mathbb{P}(13,6,4,11) \\
\text { Diff }=\left(0,0,0, \frac{2}{3}\right)\end{array}$ & 6 \\
\hline 66 & $z x^{5}+x y^{7}$ & $\begin{array}{c}t+z^{3} x^{2}+z x^{5}+x y \subset \mathbb{P}(13,3,2,11) \\
\mathbb{P}(3,2,11), \text { Diff }=\left(\frac{1}{2}, 0,0, \frac{6}{7}\right)\end{array}$ & 22 \\
\hline 67 & $z^{2} y^{4}+a z x^{5}+b x^{3} y^{4}$ & $\begin{array}{c}t^{2}+z^{3} x^{2}+g\left(z, x, y^{1 / 2}\right) \subset \mathbb{P}(13,6,4,7) \\
\text { Diff }=\left(0,0,0, \frac{1}{2}\right)\end{array}$ & 7 \\
\hline 68 & $z x^{4} y+y^{7}$ & $\begin{array}{c}t^{2}+z^{3} x^{2}+g(z, x, y) \subset \mathbb{P}(35,16,11,10) \\
\text { Diff }=\varnothing\end{array}$ & 5 \\
\hline 69 & $z x^{4} y+y^{8}$ & $\begin{array}{c}t^{2}+z^{3} x+z x^{2} y+y^{8} \subset \mathbb{P}(20,9,13,5) \\
\text { Diff }=\left(0,0, \frac{1}{2}, 0\right)\end{array}$ & 10 \\
\hline 70 & $\begin{array}{c}z x^{4} y+x y^{n} \\
n=6 ; 7\end{array}$ & $\begin{array}{c}t+z^{3} x^{2}+z x^{4} y+x y^{n} \subset \mathbb{P}(10 n-3,2 n+1,2 n-3,8) \\
\mathbb{P}(2 n+1,2 n-3,8), \text { Diff }=\left(\frac{1}{2}, 0,0,0\right)\end{array}$ & 6,16 \\
\hline 71 & $z^{2} y^{4}+z x^{4} y+a x^{2} y^{5}$ & $\begin{array}{c}t^{2}+z^{3} x+g\left(z, x^{1 / 2}, y\right) \subset \mathbb{P}(11,5,7,3) \\
\text { Diff }=\left(0,0, \frac{1}{2}, 0\right)\end{array}$ & 6 \\
\hline 72 & $\begin{array}{c}a z^{2} x y^{3}+b z x^{4} y+c z y^{6}+d x^{3} y^{4} \\
|b|+|d| \neq 0 \&|a|+|c| \neq 0\end{array}$ & $\begin{array}{c}t+z^{3} x^{2}+g(z, x, y) \subset \mathbb{P}(31,7,5,4) \\
\mathbb{P}(7,5,4), \text { Diff }=\left(\frac{1}{2}, 0,0,0\right)\end{array}$ & 8 \\
\hline 73 & $\begin{array}{c}z x^{3} y^{2}+y^{n} \\
n=7 ; 9\end{array}$ & $\begin{array}{c}t+z^{3} x^{2}+z x^{3} y^{2}+y^{n} \subset \mathbb{P}(7 n, n+4,2 n-6,7) \\
\mathbb{P}(n+4,2 n-6,7), \text { Diff }=\left(\frac{1}{2}, 0,0,0\right)\end{array}$ & 10,14 \\
\hline 74 & $z x^{3} y^{2}+y^{8}$ & $\begin{array}{c}t^{2}+z^{3} x^{2}+z x^{3} y+y^{4} \subset \mathbb{P}(14,6,5,7) \\
\text { Diff }=\left(0,0,0, \frac{1}{2}\right)\end{array}$ & 7 \\
\hline 75 & $a z^{2} y^{5}+z x^{3} y^{2}+b x y^{7}$ & $\begin{array}{c}t+z^{3} x^{2}+g(z, x, y) \subset \mathbb{P}(43,9,8,5) \\
\quad \mathbb{P}(9,8,5), \text { Diff }=\left(\frac{1}{2}, 0,0,0\right)\end{array}$ & 10 \\
\hline
\end{tabular}

ТАБлицА 9. Особенность: $t^{2}+z^{3} y^{2}+g(z, x, y)$

\begin{tabular}{|c|c|c|c|}
\hline 1 & $x^{7}+y^{n}$ & $t+z y^{2}+x+y^{n} \subset \mathbb{P}(n, n-2, n, 1)$ & 28,42 \\
& $n=9 ; 13$ & $\mathbb{P}(n-2, n, 1)$, Diff $=\left(\frac{1}{2}, \frac{2}{3}, \frac{6}{7}, 0\right)$ & \\
\hline
\end{tabular}


Продолжение таблицы 9

\begin{tabular}{|c|c|c|c|}
\hline 2 & $x^{7}+y^{10}$ & $\begin{array}{c}t^{2}+z y^{2}+x+y^{10} \subset \mathbb{P}(5,8,10,1) \\
\mathbb{P}(5,8,1), \text { Diff }=\left(0, \frac{2}{3}, \frac{6}{7}, 0\right)\end{array}$ & 15 \\
\hline 3 & $\begin{array}{c}x^{7}+a y^{5} z^{i} f_{2-i}\left(z, y^{3}\right) \\
i \leqslant 1\end{array}$ & $\begin{array}{c}t+z^{3} y^{2}+g\left(z, x^{1 / 7}, y\right) \subset \mathbb{P}(11,3,11,1) \\
\mathbb{P}(3,11,1), \text { Diff }=\left(\frac{1}{2}, 0, \frac{6}{7}, 0\right)\end{array}$ & 14 \\
\hline 4 & $x^{7}+y^{12}$ & $\begin{array}{l}t^{2}+z y+x+y^{6} \subset \mathbb{P}(3,5,6,1) \\
\mathbb{P}(3,5,1), \text { Diff }=\left(0, \frac{2}{3}, \frac{6}{7}, \frac{1}{2}\right)\end{array}$ & 21 \\
\hline 5 & $\begin{array}{c}x^{7}+x y^{n} \\
n=7 ; 11\end{array}$ & $\begin{array}{l}t+z y^{2}+x^{7}+x y^{n} \subset \mathbb{P}(7 n, 7 n-12, n, 6) \\
\mathbb{P}(7 n-12, n, 6), \text { Diff }=\left(\frac{1}{2}, \frac{2}{3}, 0,0\right)\end{array}$ & 22,36 \\
\hline 6 & $\begin{array}{c}x^{3 i+1} f_{2-i}\left(x^{3}, y^{4}\right) \\
i \leqslant 1\end{array}$ & $\begin{array}{c}t^{2}+z y+g\left(x, y^{1 / 2}\right) \subset \mathbb{P}(7,11,2,3) \\
\text { Diff }=\left(0, \frac{2}{3}, 0, \frac{1}{2}\right)\end{array}$ & 9 \\
\hline 7 & $\begin{array}{c}x^{2 i+1} f_{3-i}\left(x^{2}, y^{3}\right) \\
i \leqslant 2\end{array}$ & $\begin{array}{c}t+z y^{2}+g(x, y) \subset \mathbb{P}(21,17,3,2) \\
\mathbb{P}(17,3,2), \text { Diff }=\left(\frac{1}{2}, \frac{2}{3}, 0,0\right)\end{array}$ & 12 \\
\hline 8 & $\begin{array}{c}x^{3 i+1} f_{2-i}\left(x^{3}, y^{5}\right) \\
i \leqslant 1\end{array}$ & $\begin{array}{l}t+z y^{2}+g(x, y) \subset \mathbb{P}(35,29,5,3) \\
\mathbb{P}(29,5,3), \text { Diff }=\left(\frac{1}{2}, \frac{2}{3}, 0,0\right)\end{array}$ & 18 \\
\hline 9 & $x^{7}+x^{2} y^{6}$ & $\begin{array}{c}t^{2}+z y^{2}+x^{7}+x^{2} y^{6} \subset \mathbb{P}(21,32,6,5) \\
\text { Diff }=\left(0, \frac{2}{3}, 0,0\right)\end{array}$ & 9 \\
\hline 10 & $a z x^{3} y^{3}+x^{7}+b x^{2} y^{7}$ & $\begin{array}{c}t+z^{3} y^{2}+g(z, x, y) \subset \mathbb{P}(49,13,7,5) \\
\mathbb{P}(13,7,5), \text { Diff }=\left(\frac{1}{2}, 0,0,0\right)\end{array}$ & 10 \\
\hline 11 & $x^{7}+x^{2} y^{8}$ & $\begin{array}{c}t^{2}+z y+x^{7}+x^{2} y^{4} \subset \mathbb{P}(14,23,4,5) \\
\text { Diff }=\left(0, \frac{2}{3}, 0, \frac{1}{2}\right)\end{array}$ & 12 \\
\hline 12 & $x^{7}+x^{2} y^{9}$ & $\begin{array}{c}t+z y^{2}+x^{7}+x^{2} y^{9} \subset \mathbb{P}(63,53,9,5) \\
\mathbb{P}(53,9,5), \text { Diff }=\left(\frac{1}{2}, \frac{2}{3}, 0,0\right)\end{array}$ & 30 \\
\hline 13 & $b z x^{2} y^{4}+x^{7}+c x^{3} y^{5}$ & $\begin{array}{c}t+z^{3} y^{2}+g(z, x, y) \subset \mathbb{P}(35,9,5,4) \\
\mathbb{P}(9,5,4), \text { Diff }=\left(\frac{1}{2}, 0,0,0\right)\end{array}$ & 8 \\
\hline 14 & $x^{7}+x^{3} y^{7}$ & $\begin{array}{c}t+z y^{2}+x^{7}+x^{3} y^{7} \subset \mathbb{P}(49,41,7,4) \\
\mathbb{P}(41,7,4), \text { Diff }=\left(\frac{1}{2}, \frac{2}{3}, 0,0\right)\end{array}$ & 24 \\
\hline 15 & $\begin{array}{c}x^{7}+z y^{n} \\
n=7 ; 9\end{array}$ & $\begin{array}{c}t+z^{3} y^{2}+x+z y^{n} \subset \mathbb{P}(3 n-2, n-2,3 n-2,2) \\
\mathbb{P}(n-2,3 n-2,2), \text { Diff }=\left(\frac{1}{2}, 0, \frac{6}{7}, 0\right)\end{array}$ & 22,28 \\
\hline 16 & $\begin{array}{c}x^{7}+z x y^{n} \\
n=5 ; 7\end{array}$ & $\begin{array}{c}t+z^{3} y^{2}+x^{7}+z x y^{n} \subset \mathbb{P}(21 n-14,7 n-12,3 n-2,11) \\
\mathbb{P}(7 n-12,3 n-2,11), \text { Diff }=\left(\frac{1}{2}, 0,0,0\right)\end{array}$ & 16,22 \\
\hline 17 & $x^{7}+z x y^{6}$ & $\begin{array}{c}t^{2}+z^{3} y+x^{7}+z x y^{3} \subset \mathbb{P}(28,15,8,11) \\
\text { Diff }=\left(0,0,0, \frac{1}{2}\right)\end{array}$ & 11 \\
\hline 18 & $x^{7}+z x^{2} y^{5}$ & $\begin{array}{c}t+z^{3} y^{2}+x^{7}+z x^{2} y^{5} \subset \mathbb{P}(91,25,13,8) \\
\mathbb{P}(25,13,8), \text { Diff }=\left(\frac{1}{2}, 0,0,0\right)\end{array}$ & 16 \\
\hline 19 & $\begin{array}{c}z x^{5}+y^{n} \\
n=7 ; 13\end{array}$ & $\begin{array}{l}t+z^{3} y^{2}+z x+y^{n} \subset \mathbb{P}(3 n, n-2,2 n+2,3) \\
\quad \mathbb{P}(n-2,2 n+2,3), \text { Diff }=\left(\frac{1}{2}, 0, \frac{4}{5}, 0\right)\end{array}$ & 15,30 \\
\hline 20 & $\begin{array}{c}z x^{5}+c z x^{2} y^{4}+ \\
+e x^{6} y+d x^{3} y^{5}+l y^{9} \\
|c|+|d|+|l| \neq 0\end{array}$ & $\begin{array}{c}t+z^{3} y^{2}+g(z, x, y) \subset \mathbb{P}(27,7,4,3) \\
\mathbb{P}(7,4,3), \text { Diff }=\left(\frac{1}{2}, 0,0,0\right)\end{array}$ & 6 \\
\hline
\end{tabular}


Продолжение таблицы 9

\begin{tabular}{|c|c|c|c|}
\hline 21 & $z x^{5}+y^{10}$ & $\begin{array}{c}t^{2}+z^{3} y^{2}+z x+y^{10} \subset \mathbb{P}(15,8,22,3) \\
\text { Diff }=\left(0,0, \frac{4}{5}, 0\right)\end{array}$ & 11 \\
\hline 22 & $\begin{array}{l}b y^{5} z^{i} f_{3-i}\left(z, y^{3}\right)+z x^{5}+ \\
\quad+a x^{5} y^{3}, i \leqslant 2 \| a \neq 0\end{array}$ & $\begin{array}{c}t+z^{3} y^{2}+g\left(z, x^{1 / 5}, y\right) \subset \mathbb{P}(11,3,8,1) \\
\mathbb{P}(3,8,1), \text { Diff }=\left(\frac{1}{2}, 0, \frac{4}{5}, 0\right)\end{array}$ & 10 \\
\hline 23 & $z x^{5}+y^{12}$ & $\begin{array}{c}t^{2}+z^{3} y^{2}+z x+y^{6} \subset \mathbb{P}(9,5,13,3) \\
\text { Diff }=\left(0,0, \frac{4}{5}, \frac{1}{2}\right)\end{array}$ & 15 \\
\hline 24 & $\begin{array}{c}z x^{5}+x y^{4 n+2} \\
n=1 ; 2\end{array}$ & $\begin{array}{c}t^{2}+z^{3} y+g\left(z, x, y^{1 / 2}\right) \subset \mathbb{P}(15 n+8,10 n+1,4 n+3,13) \\
\text { Diff }=\left(0,0,0, \frac{1}{2}\right)\end{array}$ & 9,13 \\
\hline 25 & $\begin{array}{l}z x^{5}+x y^{n} \\
n=7 ; 9 ; 11\end{array}$ & $\begin{array}{c}t+z^{3} y^{2}+z x^{5}+x y^{n} \subset \mathbb{P}(15 n+2,5 n-8,2 n+2,13) \\
\mathbb{P}(5 n-8,2 n+2,13), \text { Diff }=\left(\frac{1}{2}, 0,0,0\right)\end{array}$ & $16,22,26$ \\
\hline 26 & $z x^{5}+x y^{8}$ & $\begin{array}{c}t^{2}+z^{3} y^{2}+g(z, x, y) \subset \mathbb{P}(61,32,18,13) \\
\text { Diff }=\varnothing\end{array}$ & 9 \\
\hline 27 & $\begin{array}{c}z x^{5}+x^{2} y^{n} \\
n=5 ; 7 ; 9\end{array}$ & $\begin{array}{c}t+z^{3} y^{2}+z x^{5}+x^{2} y^{n} \subset \mathbb{P}(15 n+4,5 n-6,2 n+2,11) \\
\mathbb{P}(5 n-6,2 n+2,11), \text { Diff }=\left(\frac{1}{2}, 0,0,0\right)\end{array}$ & $12,18,22$ \\
\hline 28 & $z x^{5}+x^{2} y^{6}$ & $\begin{array}{c}t^{2}+z^{3} y^{2}+g(z, x, y) \subset \mathbb{P}(47,24,14,11) \\
\text { Diff }=\varnothing\end{array}$ & 7 \\
\hline 29 & $z x^{5}+x^{2} y^{8}$ & $\begin{array}{c}t^{2}+z^{3} y+z x^{5}+x^{2} y^{4} \subset \mathbb{P}(31,17,9,11) \\
\text { Diff }=\left(0,0,0, \frac{1}{2}\right)\end{array}$ & 11 \\
\hline 30 & $z x^{5}+x^{3} y^{4}$ & $\begin{array}{c}t^{2}+z^{3} y^{2}+g(z, x, y) \subset \mathbb{P}(33,16,10,9) \\
\text { Diff }=\varnothing\end{array}$ & 5 \\
\hline 31 & $z x^{5}+x^{3} y^{6}$ & $\begin{array}{c}t^{2}+z^{3} y+z x^{5}+x^{3} y^{3} \subset \mathbb{P}(24,13,7,9) \\
\text { Diff }=\left(0,0,0, \frac{1}{2}\right)\end{array}$ & 9 \\
\hline 32 & $z x^{5}+x^{3} y^{7}$ & $\begin{array}{c}t+z^{3} y^{2}+z x^{5}+x^{3} y^{7} \subset \mathbb{P}(111,31,16,9) \\
\mathbb{P}(31,16,9), \text { Diff }=\left(\frac{1}{2}, 0,0,0\right)\end{array}$ & 18 \\
\hline 33 & $\begin{array}{c}z x^{5}+x^{4} y^{n} \\
n=3 ; 5\end{array}$ & $\begin{array}{c}t+z^{3} y^{2}+z x^{5}+x^{4} y^{n} \subset \mathbb{P}(15 n+8,5 n-2,2 n+2,7) \\
\mathbb{P}(5 n-2,2 n+2,7), \text { Diff }=\left(\frac{1}{2}, 0,0,0\right)\end{array}$ & 10,14 \\
\hline 34 & $z x^{5}+x^{4} y^{4}$ & $\begin{array}{c}t^{2}+z^{3} y+z x^{5}+x^{4} y^{2} \subset \mathbb{P}(17,9,5,7) \\
\text { Diff }=\left(0,0,0, \frac{1}{2}\right)\end{array}$ & 7 \\
\hline
\end{tabular}

ТАБлицА 10 . Особенность: $t^{2}+z^{3} x y+g(z, x, y)$

\begin{tabular}{|c|c|c|c|}
\hline 1 & $x^{5}+y^{2 n}$ & $t^{2}+z x y+g(x, y) \subset \mathbb{P}(5 n, 8 n-5,2 n, 5)$ & $7,9,15$ \\
& $n=3 ; 6 ; 8,9$ & Diff $=\left(0, \frac{2}{3}, 0,0\right)$ & \\
\hline 2 & $x^{5}+y^{n}$ & $t+z x y+x^{5}+y^{n} \subset \mathbb{P}(5 n, 4 n-5, n, 5)$ & $10,14,22$ \\
& $n=7 ; 9 ; 13 ; 19$ & $\mathbb{P}(4 n-5, n, 5)$, Diff $=\left(\frac{1}{2}, \frac{2}{3}, 0,0\right)$ & 30 \\
\hline 3 & $a z x^{2} y^{2 n+1}+x^{5}+b y^{6 n+2}$ & $t^{2}+z^{3} x y+g(z, x, y) \subset \mathbb{P}(15 n+5,8 n+1,6 n+2,5)$ & 4,5 \\
& $n=1 b \neq 0 ; 2$ & $\operatorname{Diff}=\varnothing$ & \\
\hline 4 & $x^{i}\left(x+y^{2}\right)^{j} f_{5-i-j}\left(x, y^{2}\right)$ & $t^{2}+z x y+g(x, y) \subset \mathbb{P}(5,7,2,1)$ & 3 \\
& $i \leqslant 2 \& j \leqslant 4$ & $\operatorname{Diff}=\left(0, \frac{2}{3}, 0,0\right)$ & \\
\hline
\end{tabular}


Продолжение таблицы 10

\begin{tabular}{|c|c|c|c|}
\hline 5 & $\begin{array}{c}a z x^{2} y^{2 n}+x^{5}+b y^{6 n-1} \\
n=2 ; 3\end{array}$ & $\begin{array}{c}t+z^{3} x y+g(z, x, y) \subset \mathbb{P}(30 n-5,8 n-3,6 n-1,5) \\
\mathbb{P}(8 n-3,6 n-1,5), \text { Diff }=\left(\frac{1}{2}, 0,0,0\right)\end{array}$ & 10 \\
\hline 6 & $\begin{array}{c}x^{i} f_{5-i}\left(x, y^{3}\right) \\
i \leqslant 3\end{array}$ & $\begin{array}{c}t+z x y+g(x, y) \subset \mathbb{P}(15,11,3,1) \\
\mathbb{P}(11,3,1), \text { Diff }=\left(\frac{1}{2}, \frac{2}{3}, 0,0\right)\end{array}$ & 6 \\
\hline 7 & $\begin{array}{c}x^{5}+x^{2} y^{2 n} \\
n=4,5\end{array}$ & $\begin{array}{c}t^{2}+z x y+g(x, y) \subset \mathbb{P}(5 n, 8 n-3,2 n, 3) \\
\text { Diff }=\left(0, \frac{2}{3}, 0,0\right)\end{array}$ & 9 \\
\hline 8 & $\begin{array}{l}x^{5}+x^{2} y^{n} \\
n=5,7 ; 11\end{array}$ & $\begin{array}{c}t+z x y+g(x, y) \subset \mathbb{P}(5 n, 4 n-3, n, 3) \\
\mathbb{P}(4 n-3, n, 3), \text { Diff }=\left(\frac{1}{2}, \frac{2}{3}, 0,0\right)\end{array}$ & 10,18 \\
\hline 9 & $\begin{array}{l}x^{5}+z y^{2 n+1} \\
n=2 ; 3 ; 4,5,6\end{array}$ & $\begin{array}{c}t^{2}+z^{3} x y+g(z, x, y) \subset \mathbb{P}(15 n+5,8 n-1,6 n+2,11) \\
\text { Diff }=\varnothing\end{array}$ & $3,5,11$ \\
\hline 10 & $\begin{aligned} & x^{5}+z y^{n} \\
n= & 6 ; 8,10 ; 12,14\end{aligned}$ & $\begin{array}{c}t+z^{3} x y+g(z, x, y) \subset \mathbb{P}(15 n-5,4 n-5,3 n-1,11) \\
\mathbb{P}(4 n-5,3 n-1,11), \text { Diff }=\left(\frac{1}{2}, 0,0,0\right)\end{array}$ & $8,14,22$ \\
\hline 11 & $\begin{array}{c}x^{5}+z^{2} y^{2 n} \\
n=2 ; 3,4\end{array}$ & $\begin{array}{c}t^{2}+z^{3} x y+g(z, x, y) \subset \mathbb{P}(15 n-5,8 n-5,6 n-2,7) \\
\text { Diff }=\varnothing\end{array}$ & 6,7 \\
\hline 12 & $\begin{array}{l}x^{5}+z^{2} y^{n} \\
n=5 ; 7,9\end{array}$ & $\begin{array}{c}t+z^{3} x y+g(z, x, y) \subset \mathbb{P}(15 n-10,4 n-5,3 n-2,7) \\
\mathbb{P}(4 n-5,3 n-2,7), \text { Diff }=\left(\frac{1}{2}, 0,0,0\right)\end{array}$ & 6,14 \\
\hline 13 & $\begin{array}{c}x^{i} y^{j}(x+y)^{l} f_{6-i-j-l}(x, y) \\
i \leqslant 1 \& j \leqslant 1 \& l \leqslant 3\end{array}$ & $\begin{array}{c}t^{2}+z x y+g(x, y) \subset \mathbb{P}(3,4,1,1) \\
\text { Diff }=\left(0, \frac{2}{3}, 0,0\right)\end{array}$ & 3 \\
\hline 14 & $\begin{array}{l}x^{6}+y^{n} \\
n=7 ; 11\end{array}$ & $\begin{array}{c}t^{2}+z x y+g(x, y) \subset \mathbb{P}(3 n, 5 n-6, n, 6) \\
\text { Diff }=\left(0, \frac{2}{3}, 0,0\right)\end{array}$ & 10,18 \\
\hline 15 & $\begin{array}{c}x^{3 i} f_{2-i}\left(x^{3}, y^{n}\right) \\
n=4 i=0 ; 5\end{array}$ & $\begin{array}{c}t^{2}+z x y+g(x, y) \subset \mathbb{P}(3 n, 5 n-3, n, 3) \\
\text { Diff }=\left(0, \frac{2}{3}, 0,0\right)\end{array}$ & 6,9 \\
\hline 16 & $\begin{array}{c}x^{2 i} f_{3-i}\left(x^{2}, y^{3}\right) \\
i \leqslant 1\end{array}$ & $\begin{array}{c}t^{2}+z x y+g(x, y) \subset \mathbb{P}(9,13,3,2) \\
\text { Diff }=\left(0, \frac{2}{3}, 0,0\right)\end{array}$ & 6 \\
\hline 17 & $\begin{array}{c}a z^{2} y^{4}+b z x^{3} y^{2}+x^{6}+ \\
+c x^{2} y^{5},|a|+|c| \neq 0\end{array}$ & $\begin{array}{c}t^{2}+z^{3} x y+g(z, x, y) \subset \mathbb{P}(15,7,5,4) \\
\text { Diff }=\varnothing\end{array}$ & 4 \\
\hline 18 & $x^{6}+x^{2} y^{7}$ & $\begin{array}{c}t^{2}+z x y+g(x, y) \subset \mathbb{P}(21,31,7,4) \\
\text { Diff }=\left(0, \frac{2}{3}, 0,0\right)\end{array}$ & 12 \\
\hline 19 & $\begin{array}{l}x^{6}+z y^{n} \\
n=5 ; 6 ; 7 ; 8\end{array}$ & $\begin{array}{c}t^{2}+z^{3} x y+g(z, x, y) \subset \mathbb{P}(9 n-3,5 n-6,3 n-1,13) \\
\text { Diff }=\varnothing\end{array}$ & $\begin{array}{c}7,8,10 \\
13\end{array}$ \\
\hline 20 & $x^{6}+z x^{2} y^{4}$ & $\begin{array}{c}t^{2}+z^{3} x y+g(z, x, y) \subset \mathbb{P}(33,16,11,7) \\
\text { Diff }=\varnothing\end{array}$ & 7 \\
\hline 21 & $x^{6}+z^{2} y^{5}$ & $\begin{array}{c}t^{2}+z^{3} x y+g(z, x, y) \subset \mathbb{P}(39,19,13,8) \\
\text { Diff }=\varnothing\end{array}$ & 8 \\
\hline 22 & $\begin{array}{c}x^{i} y^{j}(x+y)^{l} f_{7-i-j-l}(x, y) \\
i \leqslant 2 \& j \leqslant 2 \& l \leqslant 4\end{array}$ & $\begin{array}{c}t+z x y+g(x, y) \subset \mathbb{P}(7,5,1,1) \\
\mathbb{P}(5,1,1), \text { Diff }=\left(\frac{1}{2}, \frac{2}{3}, 0,0\right)\end{array}$ & 6 \\
\hline 23 & $x^{7}+y^{8}$ & $\begin{array}{c}t^{2}+z x y+x^{7}+y^{8} \subset \mathbb{P}(28,41,8,7) \\
\text { Diff }=\left(0, \frac{2}{3}, 0,0\right)\end{array}$ & 12 \\
\hline
\end{tabular}


Продолжение таблицы 10

\begin{tabular}{|c|c|c|c|}
\hline 24 & $x^{7}+y^{9}$ & $\begin{array}{c}t+z x y+x^{7}+y^{9} \subset \mathbb{P}(63,47,9,7) \\
\mathbb{P}(47,9,7), \text { Diff }=\left(\frac{1}{2}, \frac{2}{3}, 0,0\right)\end{array}$ & 42 \\
\hline 25 & $x^{7}+x^{2} y^{6}$ & $\begin{array}{c}t^{2}+z x y+x^{7}+x^{2} y^{6} \subset \mathbb{P}(21,31,6,5) \\
\text { Diff }=\left(0, \frac{2}{3}, 0,0\right)\end{array}$ & 15 \\
\hline 26 & $x^{7}+z y^{6}$ & $\begin{array}{c}t+z^{3} x y+x^{7}+z y^{6} \subset \mathbb{P}(119,29,17,15) \\
\mathbb{P}(29,17,15), \text { Diff }=\left(\frac{1}{2}, 0,0,0\right)\end{array}$ & 10 \\
\hline 27 & $x^{7}+z x^{2} y^{4}$ & $\begin{array}{c}t+z^{3} x y+x^{7}+z x^{2} y^{4} \subset \mathbb{P}(77,19,11,9) \\
\mathbb{P}(19,11,9), \text { Diff }=\left(\frac{1}{2}, 0,0,0\right)\end{array}$ & 18 \\
\hline 28 & $x^{7}+z^{2} y^{4}$ & $\begin{array}{c}t^{2}+z^{3} x y+x^{7}+z^{2} y^{4} \subset \mathbb{P}(35,17,10,9) \\
\text { Diff }=\varnothing\end{array}$ & 9 \\
\hline 29 & $\begin{array}{c}x^{5} y^{2}+y^{2 n} \\
n=4 ; 5\end{array}$ & $\begin{array}{c}t^{2}+z x y+x^{5} y^{2}+y^{2 n} \subset \mathbb{P}(5 n, 8 n-3,2 n-2,5) \\
\text { Diff }=\left(0, \frac{2}{3}, 0,0\right)\end{array}$ & 9,15 \\
\hline 30 & $a z x^{2} y^{4}+x^{5} y^{2}+b y^{9}$ & $\begin{array}{c}t+z^{3} x y+g(z, x, y) \subset \mathbb{P}(45,11,7,5) \\
\mathbb{P}(11,7,5), \text { Diff }=\left(\frac{1}{2}, 0,0,0\right)\end{array}$ & 10 \\
\hline 31 & $x^{5} y^{2}+y^{11}$ & $\begin{array}{c}t+z x y+x^{5} y^{2}+y^{11} \subset \mathbb{P}(55,41,9,5) \\
\text { Diff }=\left(\frac{1}{2}, \frac{2}{3}, 0,0\right)\end{array}$ & 30 \\
\hline 32 & $x^{5} y^{2}+x^{2} y^{6}$ & $\begin{array}{c}t^{2}+z x y+x^{5} y^{2}+x^{2} y^{6} \subset \mathbb{P}(13,19,4,3) \\
\text { Diff }=\left(0, \frac{2}{3}, 0,0\right)\end{array}$ & 9 \\
\hline 33 & $x^{5} y^{2}+x^{2} y^{7}$ & $\begin{array}{c}t+z x y+x^{5} y^{2}+x^{2} y^{7} \subset \mathbb{P}(31,23,5,3) \\
\mathbb{P}(23,5,3), \text { Diff }=\left(\frac{1}{2}, \frac{2}{3}, 0,0\right)\end{array}$ & 18 \\
\hline 34 & $\begin{array}{c}x^{5} y^{2}+z y^{n} \\
n=6 ; 8\end{array}$ & $\begin{array}{c}t+z^{3} x y+g(z, x, y) \subset \mathbb{P}(15 n-3,4 n-3,3 n-5,11) \\
\mathbb{P}(4 n-3,3 n-5,11), \text { Diff }=\left(\frac{1}{2}, 0,0,0\right)\end{array}$ & 16,22 \\
\hline 35 & $x^{5} y^{2}+z y^{7}$ & $\begin{array}{c}t^{2}+z^{3} x y+x^{5} y^{2}+z y^{7} \subset \mathbb{P}(51,25,16,11) \\
\text { Diff }=\varnothing\end{array}$ & 11 \\
\hline 36 & $x^{5} y^{2}+z^{2} y^{4}$ & $\begin{array}{c}t^{2}+z^{3} x y+x^{5} y^{2}+z^{2} y^{4} \subset \mathbb{P}(27,13,8,7) \\
\text { Diff }=\varnothing\end{array}$ & 7 \\
\hline 37 & $x^{5} y^{2}+z^{2} y^{5}$ & $\begin{array}{c}t+z^{3} x y+x^{5} y^{2}+z^{2} y^{5} \subset \mathbb{P}(69,17,11,7) \\
\mathbb{P}(17,11,7), \text { Diff }=\left(\frac{1}{2}, 0,0,0\right)\end{array}$ & 14 \\
\hline 38 & $\begin{array}{l}z x^{5}+y^{n} \\
n=7 ; 8 ; 9\end{array}$ & $\begin{array}{c}t^{2}+z^{3} x y+z x^{5}+y^{n} \subset \mathbb{P}(7 n, 4 n-5,2 n+1,14) \\
\text { Diff }=\varnothing\end{array}$ & $5,7,14$ \\
\hline 39 & $\begin{array}{c}z x^{5}+x^{2} y^{n} \\
n=5 ; 6\end{array}$ & $\begin{array}{c}t^{2}+z^{3} x y+z x^{5}+x^{2} y^{n} \subset \mathbb{P}(7 n+1,4 n-3,2 n+1,10) \\
\text { Diff }=\varnothing\end{array}$ & 5,6 \\
\hline 40 & $z^{2} y^{4}+z x^{5}+b x^{4} y^{3}$ & $\begin{array}{c}t^{2}+z^{3} x y+g(z, x, y) \subset \mathbb{P}(23,11,7,6) \\
\text { Diff }=\varnothing\end{array}$ & 6 \\
\hline
\end{tabular}

ТАБЛИЦА 11. Особенность: $t^{2}+f(z, x)+g(z, x, y)=t^{2}+z^{i} x^{j} f_{5-i-j}(z, x)+g(z, x, y), i \leqslant j$

\begin{tabular}{|c|c|c|c|}
\hline 1 & $y^{2 n}$ & $t^{2}+f(z, x)+y^{2} \subset \mathbb{P}(5,2,2,5)$ & 3,5 \\
& $n=3 j=1 ; 4 j \leqslant 2$ & Diff $=\left(0,0,0, \frac{n-1}{n}\right)$ & \\
\hline
\end{tabular}


Продолжение таблицы 11

\begin{tabular}{|c|c|c|c|}
\hline 2 & $\begin{array}{c}y^{n} \\
(j \leqslant 2) \&(n=7 ; 9)\end{array}$ & $\begin{array}{c}t+f(z, x)+y \subset \mathbb{P}(5,1,1,5) \\
\mathbb{P}(1,1,5), \text { Diff }=\left(\frac{1}{2}, 0,0, \frac{n-1}{n}\right)\end{array}$ & 8,10 \\
\hline 3 & $\begin{array}{c}z y^{n}, n=5 \& j=1 \\
n=7 \& i \leqslant 2 \& j \leqslant 3\end{array}$ & $\begin{array}{c}t+f(z, x)+z y \subset \mathbb{P}(5,1,1,4) \\
\mathbb{P}(1,1,4), \text { Diff }=\left(\frac{1}{2}, 0,0, \frac{n-1}{n}\right)\end{array}$ & 6,8 \\
\hline $\begin{array}{c}4 \\
(1)\end{array}$ & $y^{3} f_{3}^{\prime}(z, x)+z y^{6}$ & $\begin{array}{c}t+f(z, x)+g\left(z, x, y^{1 / 3}\right) \subset \mathbb{P}(5,1,1,2) \\
\mathbb{P}(1,1,2), \text { Diff }=\left(\frac{1}{2}, 0,0, \frac{2}{3}\right)\end{array}$ & 6 \\
\hline 5 & $\begin{array}{c}z x y^{4} \mid \| z^{2} y^{4} \\
j \leqslant 1 \mid \|(i \leqslant 1 \& j \leqslant 2)\end{array}$ & $\begin{array}{c}t^{2}+f(z, x)+g\left(z, x, y^{1 / 2}\right) \subset \mathbb{P}(5,2,2,3) \\
\operatorname{Diff}=\left(0,0,0, \frac{1}{2}\right)\end{array}$ & 3 \\
\hline 6 & $\begin{array}{c}z x y^{5} \mid \| z^{2} y^{5} \\
j \leqslant 2 \mid \|(i \leqslant 1 \& j \leqslant 2)\end{array}$ & $\begin{array}{c}t+f(z, x)+g\left(z, x, y^{1 / 5}\right) \subset \mathbb{P}(5,1,1,3) \\
\mathbb{P}(1,1,3), \text { Diff }=\left(\frac{1}{2}, 0,0, \frac{4}{5}\right)\end{array}$ & 6 \\
\hline
\end{tabular}

(1) Особенность $t^{2}+z^{i} x^{j} f_{5-i-j}(z, x)+a y^{3} z^{k} x^{l} f_{3-k-l}(z, x)+b z y^{6}$. Предполагаем, что при любой квазиоднородной замене $f_{5} \in \mathscr{M}_{1}$. Каноническая особенность этого типа является исключительной, кроме случаев: $(i=3 \& k=1) ;(i=2 \& k \geqslant 1) ;(j=3 \& l=1)$.

ТАБЛИцА 12. Особенность: $t^{2}+g(z, x, y)$, где $g_{5} \in \mathscr{M}_{1}$

\begin{tabular}{|c|c|c|c|}
\hline 1 & $\begin{array}{c}z^{5}+z x^{3} y+y^{2 n} \\
n=3 ; 6 ; 8,9\end{array}$ & $\begin{array}{c}t^{2}+z^{5}+z x y+y^{2 n} \subset \mathbb{P}(5 n, 2 n, 8 n-5,5) \\
\text { Diff }=\left(0,0, \frac{2}{3}, 0\right)\end{array}$ & $7,9,15$ \\
\hline 2 & $\begin{array}{c}z^{5}+z x^{3} y+y^{n} \\
n=7 ; 9 ; 13 ; 19\end{array}$ & $\begin{array}{c}t+z^{5}+z x y+y^{n} \subset \mathbb{P}(5 n, n, 4 n-5,5) \\
\mathbb{P}(n, 4 n-5,5), \text { Diff }=\left(\frac{1}{2}, 0, \frac{2}{3}, 0\right)\end{array}$ & $\begin{array}{c}10,14,22 \\
30\end{array}$ \\
\hline 3 & $\begin{array}{l}z^{5}+z x^{3} y+a z^{2} x y^{2 n+1}+ \\
+b y^{6 n+2}, n=1 b \neq 0 ; 2\end{array}$ & $\begin{array}{c}t^{2}+g(z, x, y) \subset \mathbb{P}(15 n+5,6 n+2,8 n+1,5) \\
\text { Diff }=\varnothing\end{array}$ & 4,5 \\
\hline 4 & $\begin{array}{c}z^{5}+z x^{3} y+y^{4} z^{i} f_{3-i}\left(z, y^{2}\right)+ \\
a x^{3} y^{3}, i \leqslant 2 \| a \neq 0\end{array}$ & $\begin{array}{c}t^{2}+g\left(z, x^{1 / 3}, y\right) \subset \mathbb{P}(5,2,7,1) \\
\text { Diff }=\left(0,0, \frac{2}{3}, 0\right)\end{array}$ & 3 \\
\hline 5 & $\begin{array}{c}z^{5}+z x^{3} y+a z^{2} x y^{n+1}+ \\
b y^{3 n+2}, n=3,5\end{array}$ & $\begin{array}{c}t+g(z, x, y) \subset \mathbb{P}(15 n+10,3 n+2,4 n+1,5) \\
\mathbb{P}(3 n+2,4 n+1,5), \text { Diff }=\left(\frac{1}{2}, 0,0,0\right)\end{array}$ & 10 \\
\hline 6 & $\begin{array}{c}z^{5}+z x^{3} y+b y^{6} z^{i} f_{3-i}\left(z, y^{3}\right)+ \\
a x^{3} y^{4}\end{array}$ & $\begin{array}{c}t+g\left(z, x^{1 / 3}, y\right) \subset \mathbb{P}(15,3,11,1) \\
\mathbb{P}(3,11,1), \text { Diff }=\left(\frac{1}{2}, 0, \frac{2}{3}, 0\right)\end{array}$ & 6 \\
\hline 7 & $\begin{array}{c}z^{5}+z x^{3} y+x y^{2 n+1} \\
n=2 ; 3 ; 4,5,6\end{array}$ & $\begin{array}{c}t^{2}+g(z, x, y) \subset \mathbb{P}(15 n+5,6 n+2,8 n-1,11) \\
\text { Diff }=\varnothing\end{array}$ & $3,5,11$ \\
\hline 8 & $\begin{array}{l}z^{5}+z x^{3} y+x y^{n} \\
n=6 ; 8,10 ; 12,14\end{array}$ & $\begin{array}{c}t+g(z, x, y) \subset \mathbb{P}(15 n-5,3 n-1,4 n-5,11) \\
\mathbb{P}(3 n-1,4 n-5,11), \text { Diff }=\left(\frac{1}{2}, 0,0,0\right)\end{array}$ & $8,14,22$ \\
\hline 9 & $\begin{array}{c}z^{5}+z x^{3} y+x^{2} y^{2 n} \\
n=2 ; 3,4\end{array}$ & $\begin{array}{c}t^{2}+g(z, x, y) \subset \mathbb{P}(15 n-5,6 n-2,8 n-5,7) \\
\text { Diff }=\varnothing\end{array}$ & 6,7 \\
\hline 10 & $\begin{array}{c}z^{5}+z x^{3} y+x^{2} y^{n} \\
n=5 ; 7,9\end{array}$ & $\begin{array}{l}t+g(z, x, y) \subset \mathbb{P}(15 n-10,3 n-2,4 n-5,7) \\
\quad \mathbb{P}(3 n-2,4 n-5,7), \text { Diff }=\left(\frac{1}{2}, 0,0,0\right)\end{array}$ & 6,14 \\
\hline 11 & $\begin{array}{c}z^{5}+z x^{3} y+z^{2} y^{2 n} \\
n=4,5\end{array}$ & $\begin{array}{c}t^{2}+z^{5}+z x y+z^{2} y^{2 n} \subset \mathbb{P}(5 n, 2 n, 8 n-3,3) \\
\text { Diff }=\left(0,0, \frac{2}{3}, 0\right)\end{array}$ & 9 \\
\hline 12 & $\begin{array}{c}z^{5}+z x^{3} y+z^{2} y^{n} \\
n=5,7 ; 11\end{array}$ & $\begin{array}{c}t+z^{5}+z x y+z^{2} y^{n} \subset \mathbb{P}(5 n, n, 4 n-3,3) \\
\mathbb{P}(n, 4 n-3,3), \text { Diff }=\left(\frac{1}{2}, 0, \frac{2}{3}, 0\right)\end{array}$ & 10,18 \\
\hline
\end{tabular}


Продолжение таблицы 12

\begin{tabular}{|c|c|c|c|}
\hline 13 & $\begin{array}{c}z^{5}+x^{4} y+y^{2 n} \\
n=3,4 ; 6 ; 7\end{array}$ & $\begin{array}{c}t^{2}+z+x y+y^{2 n} \subset \mathbb{P}(n, 2 n, 2 n-1,1) \\
\mathbb{P}(n, 2 n-1,1), \text { Diff }=\left(0, \frac{4}{5}, \frac{3}{4}, 0\right)\end{array}$ & $5,12,20$ \\
\hline 14 & $\begin{array}{c}z^{5}+x^{4} y+a x^{2} y^{n+1}+ \\
b y^{2 n+1}, n=3,5\end{array}$ & $\begin{array}{c}t^{2}+g\left(z^{1 / 5}, x, y\right) \subset \mathbb{P}(2 n+1,4 n+2, n, 2) \\
\mathbb{P}(2 n+1, n, 2), \text { Diff }=\left(0, \frac{4}{5}, 0,0\right)\end{array}$ & 5 \\
\hline 15 & $\begin{array}{c}z^{5}+x^{4} y+a y^{n} x^{i} f_{3-i}\left(x, y^{n-1}\right) \\
n=3 i \leqslant 2 ; 4\end{array}$ & $\begin{array}{c}t+g\left(z^{1 / 5}, x, y\right) \subset \mathbb{P}(4 n-3,4 n-3, n-1,1) \\
\mathbb{P}(4 n-3, n-1,1), \text { Diff }=\left(\frac{1}{2}, \frac{4}{5}, 0,0\right)\end{array}$ & 6,10 \\
\hline 16 & $z^{5}+x^{4} y+a y^{4} z^{i} f_{3-i}\left(z, y^{2}\right)$ & $\begin{array}{c}t^{2}+g\left(z, x^{1 / 4}, y\right) \subset \mathbb{P}(5,2,9,1) \\
\text { Diff }=\left(0,0, \frac{3}{4}, 0\right)\end{array}$ & 4 \\
\hline 17 & $\begin{array}{c}z^{5}+x^{4} y+x y^{n} \\
n=5 ; 6,8 ; 11\end{array}$ & $\begin{array}{c}t+z+x^{4} y+x y^{n} \subset \mathbb{P}(4 n-1,4 n-1, n-1,3) \\
\mathbb{P}(4 n-1, n-1,3), \text { Diff }=\left(\frac{1}{2}, \frac{4}{5}, 0,0\right)\end{array}$ & $6,10,30$ \\
\hline 18 & $\begin{array}{c}z^{5}+x^{4} y+a z^{3} x y^{2}+b z^{2} y^{7} \\
c z x^{2} y^{4}+d x y^{9},|b|+|c|+|d| \neq 0\end{array}$ & $\begin{array}{l}t+g(z, x, y) \subset \mathbb{P}(35,7,8,3) \\
\mathbb{P}(7,8,3), \text { Diff }=\left(\frac{1}{2}, 0,0,0\right)\end{array}$ & 6 \\
\hline 19 & $\begin{array}{r}z^{5}+x^{4} y+z y^{n} \\
n=5 ; 7,9 ; 11\end{array}$ & $\begin{array}{c}t^{2}+z^{5}+x^{2} y+z y^{n} \subset \mathbb{P}(5 n, 2 n, 5 n-4,8) \\
\text { Diff }=\left(0,0, \frac{1}{2}, 0\right)\end{array}$ & $3,12,16$ \\
\hline 20 & $\begin{array}{l}z^{5}+x^{4} y+a z^{3} y^{n}+ \\
b z y^{2 n}, n=3 \quad b \neq 0 ; 5\end{array}$ & $\begin{array}{c}t^{2}+g\left(z, x^{1 / 2}, y\right) \subset \mathbb{P}(5 n, 2 n, 5 n-2,4) \\
\text { Diff }=\left(0,0, \frac{1}{2}, 0\right)\end{array}$ & 4,8 \\
\hline 21 & $\begin{array}{c}z^{5}+x^{4} y+z x y^{n} \\
n=4,5 ; 6,7 ; 8\end{array}$ & $\begin{array}{c}t+g(z, x, y) \subset \mathbb{P}(20 n-5,4 n-1,5 n-4,11) \\
\mathbb{P}(4 n-1,5 n-4,11), \text { Diff }=\left(\frac{1}{2}, 0,0,0\right)\end{array}$ & $6,18,22$ \\
\hline 22 & $\begin{array}{c}z^{5}+x^{4} y+a z^{2} y^{5}+ \\
b z x^{2} y^{3}\end{array}$ & $\begin{array}{c}t^{2}+g(z, x, y) \subset \mathbb{P}(25,10,11,6) \\
\text { Diff }=\varnothing\end{array}$ & 3 \\
\hline 23 & $\begin{array}{c}z^{5}+x^{4} y+z^{2} y^{2 n} \\
n=2 ; 3\end{array}$ & $\begin{array}{c}t^{2}+z^{5}+x y+z^{2} y^{2 n} \subset \mathbb{P}(5 n, 2 n, 10 n-3,3) \\
\text { Diff }=\left(0,0, \frac{3}{4}, 0\right)\end{array}$ & 10,12 \\
\hline 24 & $\begin{array}{c}z^{5}+x^{4} y+z^{2} x y^{n} \\
n=3,4 ; 5\end{array}$ & $\begin{array}{c}t+g(z, x, y) \subset \mathbb{P}(20 n-5,4 n-1,5 n-3,7) \\
\mathbb{P}(4 n-1,5 n-3,7), \text { Diff }=\left(\frac{1}{2}, 0,0,0\right)\end{array}$ & 10,14 \\
\hline 25 & $\begin{array}{c}z^{4} x+z x^{3} y+y^{2 n} \\
\quad n=3,4 ; 5 ; 6,7\end{array}$ & $\begin{array}{c}t^{2}+g(z, x, y) \subset \mathbb{P}(11 n, 4 n+1,6 n-4,11) \\
\text { Diff }=\varnothing\end{array}$ & $5,7,11$ \\
\hline 26 & $\begin{array}{l}z^{4} x+z x^{3} y+y^{n} \\
n=7 ; 9 ; 11 ; 13 ; 15\end{array}$ & $\begin{array}{c}t+g(z, x, y) \subset \mathbb{P}(11 n, 2 n+1,3 n-4,11) \\
\mathbb{P}(2 n+1,3 n-4,11), \text { Diff }=\left(\frac{1}{2}, 0,0,0\right)\end{array}$ & $\begin{array}{c}10,12,16 \\
18,22\end{array}$ \\
\hline 27 & $\begin{array}{c}z^{4} x+z x^{3} y+a z^{3} y^{2 n-1}+ \\
b x^{2} y^{2 n}, n=2 \quad a b \neq 0 ; 3\end{array}$ & $\begin{array}{c}t^{2}+g(z, x, y) \subset \mathbb{P}(11 n-4,4 n-1,6 n-4,5) \\
\text { Diff }=\varnothing\end{array}$ & 4,5 \\
\hline 28 & $\begin{array}{c}z^{4} x+z x^{3} y+a z^{3} y^{n-1}+ \\
b x^{2} y^{n}, n=5 \quad b \neq 0 ; 7\end{array}$ & $\begin{array}{c}t+g(z, x, y) \subset \mathbb{P}(11 n-8,2 n-1,3 n-4,5) \\
\mathbb{P}(2 n-1,3 n-4,5), \text { Diff }=\left(\frac{1}{2}, 0,0,0\right)\end{array}$ & 6,10 \\
\hline 29 & $\begin{array}{c}z^{4} x+z x^{3} y+z^{2} y^{2 n} \\
n=3,4\end{array}$ & $\begin{array}{c}t^{2}+g(z, x, y) \subset \mathbb{P}(11 n+1,4 n+1,6 n-2,7) \\
\text { Diff }=\varnothing\end{array}$ & 7 \\
\hline 30 & $\begin{array}{c}z^{4} x+z x^{3} y+z^{2} y^{n} \\
n=5 ; 7 ; 9\end{array}$ & $\begin{array}{c}t+g(z, x, y) \subset \mathbb{P}(11 n+2,2 n+1,3 n-2,7) \\
\mathbb{P}(2 n+1,3 n-2,7), \text { Diff }=\left(\frac{1}{2}, 0,0,0\right)\end{array}$ & $8,10,14$ \\
\hline 31 & $\begin{array}{c}z^{4} x+x^{4} y+y^{n} \\
n=6,8 ; 10 ; 12\end{array}$ & $\begin{array}{c}t^{2}+z x+x^{4} y+y^{n} \subset \mathbb{P}(2 n, 3 n+1, n-1,4) \\
\text { Diff }=\left(0, \frac{3}{4}, 0,0\right)\end{array}$ & $5,12,16$ \\
\hline
\end{tabular}


Продолжение таблицы 12

\begin{tabular}{|c|c|c|c|}
\hline 32 & $\begin{array}{c}z^{4} x+x^{4} y+a x^{2} y^{n+1}+ \\
b y^{2 n+1}, n=3 \quad b \neq 0 ; 5\end{array}$ & $\begin{array}{c}t^{2}+g\left(z^{1 / 4}, x, y\right) \subset \mathbb{P}(2 n+1,3 n+2, n, 2) \\
\text { Diff }=\left(0, \frac{3}{4}, 0,0\right)\end{array}$ & 4,8 \\
\hline 33 & $\begin{array}{c}z^{4} x+x^{4} y+b y^{3} x^{i} f_{3-i}\left(x, y^{2}\right) \\
+a z^{4} y^{2}, i \leqslant 2 \| a \neq 0\end{array}$ & $\begin{array}{c}t^{2}+g\left(z^{1 / 2}, x, y\right) \subset \mathbb{P}(9,7,4,2) \\
\text { Diff }=\left(0, \frac{1}{2}, 0,0\right)\end{array}$ & 4 \\
\hline 34 & $\begin{array}{l}z^{4} x+x^{4} y+z y^{n} \\
n=5 ; 6,7,8 ; 9 ; 10\end{array}$ & $\begin{array}{c}t+g(z, x, y) \subset \mathbb{P}(16 n+1,3 n+1,4 n-3,13) \\
\mathbb{P}(3 n+1,4 n-3,13), \text { Diff }=\left(\frac{1}{2}, 0,0,0\right)\end{array}$ & $\begin{array}{c}6,10,22 \\
26\end{array}$ \\
\hline 35 & $\begin{array}{c}z^{4} x+x^{4} y+a z^{2} y^{2 n-1}+ \\
b z x^{2} y^{n}, n=3 \quad a \neq 0 ; 4\end{array}$ & $\begin{array}{c}t+g(z, x, y) \subset \mathbb{P}(16 n-7,3 n-1,4 n-3,5) \\
\mathbb{P}(3 n-1,4 n-3,5), \text { Diff }=\left(\frac{1}{2}, 0,0,0\right)\end{array}$ & 6,10 \\
\hline 36 & $\begin{array}{c}z^{4} x+x^{4} y+z^{2} y^{n} \\
n=4,6\end{array}$ & $\begin{array}{c}t^{2}+g(z, x, y) \subset \mathbb{P}(8 n+1,3 n+1,4 n-2,10) \\
\text { Diff }=\varnothing\end{array}$ & 5 \\
\hline 37 & $\begin{array}{c}z^{4} x+x^{4} y+z^{3} y^{n} \\
n=4 ; 5\end{array}$ & $\begin{array}{c}t+g(z, x, y) \subset \mathbb{P}(16 n+3,3 n+1,4 n-1,7) \\
\mathbb{P}(3 n+1,4 n-1,7), \text { Diff }=\left(\frac{1}{2}, 0,0,0\right)\end{array}$ & 10,14 \\
\hline 38 & $z^{4} y+z^{2} x^{3}+y^{10}$ & $\begin{array}{c}t^{2}+z^{2} y+z x+y^{10} \subset \mathbb{P}(10,9,11,2) \\
\text { Diff }=\left(0, \frac{1}{2}, \frac{2}{3}, 0\right)\end{array}$ & 12 \\
\hline 39 & $\begin{array}{c}z^{4} y+z^{2} x^{3}+a z^{2} y^{4}+ \\
b x^{3} y^{3}+c y^{7},|a b|+|c| \neq 0\end{array}$ & $\begin{array}{c}t^{2}+g\left(z, x^{1 / 3}, y\right) \subset \mathbb{P}(7,3,8,2) \\
\text { Diff }=\left(0,0, \frac{2}{3}, 0\right)\end{array}$ & 3 \\
\hline 40 & $\begin{array}{l}z^{4} y+z^{2} x^{3}+a z^{2} x y^{3}+b x^{4} y^{2}+ \\
c x^{2} y^{5}+d y^{8},|a b|+|c|+|d| \neq 0\end{array}$ & $\begin{array}{c}t^{2}+g\left(z^{1 / 2}, x, y\right) \subset \mathbb{P}(8,7,3,2) \\
\text { Diff }=\left(0, \frac{1}{2}, 0,0\right)\end{array}$ & 4 \\
\hline 41 & $\begin{array}{c}z^{4} y+z^{2} x^{3}+c y^{5} z^{i} f_{2-i}\left(z, y^{2}\right) \\
+a z x^{3} y^{2}+b x^{3} y^{4} \\
|a|+|b|+|c| \neq 0 \& i \leqslant 1\end{array}$ & $\begin{array}{c}t+g\left(z, x^{1 / 3}, y\right) \subset \mathbb{P}(9,2,5,1) \\
\mathbb{P}(2,5,1), \text { Diff }=\left(\frac{1}{2}, 0, \frac{2}{3}, 0\right)\end{array}$ & 6 \\
\hline 42 & $\begin{array}{c}z^{4} y+z^{2} x^{3}+x y^{n} \\
n=5 ; 7\end{array}$ & $\begin{array}{c}t^{2}+g(z, x, y) \subset \mathbb{P}(6 n+1,3 n-2,2 n+2,10) \\
\text { Diff }=\varnothing\end{array}$ & 3,5 \\
\hline 43 & $\begin{array}{c}z^{4} y+z^{2} x^{3}+a z x^{2} y^{n}+ \\
b x y^{2 n}, n=3 ; 4\end{array}$ & $\begin{array}{c}t+g(z, x, y) \subset \mathbb{P}(12 n+1,3 n-1,2 n+1,5) \\
\mathbb{P}(3 n-1,2 n+1,5), \text { Diff }=\left(\frac{1}{2}, 0,0,0\right)\end{array}$ & 8,10 \\
\hline 44 & $\begin{array}{c}z^{4} y+z^{2} x^{3}+x^{2} y^{n} \\
n=4 ; 6\end{array}$ & $\begin{array}{c}t^{2}+g\left(z^{1 / 2}, x, y\right) \subset \mathbb{P}(3 n+1,3 n-1, n+1,4) \\
\text { Diff }=\left(0, \frac{1}{2}, 0,0\right)\end{array}$ & 6,8 \\
\hline 45 & $z^{4} y+z^{2} x^{3}+z y^{8}$ & $\begin{array}{c}t+z^{4} y+z^{2} x+z y^{8} \subset \mathbb{P}(31,7,17,3) \\
\mathbb{P}(7,17,3), \text { Diff }=\left(\frac{1}{2}, 0, \frac{2}{3}, 0\right)\end{array}$ & 18 \\
\hline 46 & $\begin{array}{c}z^{4} y+z^{2} x^{3}+z x y^{n} \\
n=5 ; 6\end{array}$ & $\begin{array}{c}t+g(z, x, y) \subset \mathbb{P}(12 n-1,3 n-2,2 n+1,7) \\
\mathbb{P}(3 n-2,2 n+1,7), \text { Diff }=\left(\frac{1}{2}, 0,0,0\right)\end{array}$ & 12,14 \\
\hline 47 & $\begin{array}{c}z^{3} x^{2}+z x^{3} y+y^{2 n} \\
n=4 ; 5\end{array}$ & $\begin{array}{c}t^{2}+g(z, x, y) \subset \mathbb{P}(7 n, 2 n+2,4 n-3,7) \\
\text { Diff }=\varnothing\end{array}$ & 5,7 \\
\hline 48 & $\begin{array}{c}z^{3} x^{2}+z x^{3} y+y^{n} \\
n=7 ; 9 ; 11\end{array}$ & $\begin{array}{l}t+g(z, x, y) \subset \mathbb{P}(7 n, n+2,2 n-3,7) \\
\mathbb{P}(n+2,2 n-3,7), \text { Diff }=\left(\frac{1}{2}, 0,0,0\right)\end{array}$ & $8,10,14$ \\
\hline 49 & $\begin{array}{l}z^{3} x^{2}+z x^{3} y+a z^{2} y^{2 n}+ \\
b x y^{2 n+1}, n=2 a b \neq 0 ; 3\end{array}$ & $\begin{array}{c}t^{2}+g(z, x, y) \subset \mathbb{P}(7 n+2,2 n+2,4 n-1,5) \\
\text { Diff }=\varnothing\end{array}$ & 3,5 \\
\hline 50 & $\begin{array}{c}z^{3} x^{2}+z x^{3} y+a z^{2} y^{n-1}+ \\
b x y^{n}, n=6 \quad a \neq 0 ; 8\end{array}$ & $\begin{array}{c}t+g(z, x, y) \subset \mathbb{P}(7 n-3, n+1,2 n-3,5) \\
\mathbb{P}(n+1,2 n-3,5), \text { Diff }=\left(\frac{1}{2}, 0,0,0\right)\end{array}$ & 6,10 \\
\hline
\end{tabular}


Продолжение таблицы 12

\begin{tabular}{|c|c|c|c|}
\hline 51 & $\begin{array}{c}z^{3} x^{2}+z x^{3} y+a z^{4} y^{2}+b z^{2} x y^{3}+ \\
c z y^{6}+d x^{2} y^{4},|c|+|d| \neq 0 \& \\
|a d|+|b|+|c| \neq 0\end{array}$ & $\begin{array}{c}t^{2}+g(z, x, y) \subset \mathbb{P}(11,4,5,3) \\
\text { Diff }=\varnothing\end{array}$ & 3 \\
\hline 52 & $\begin{array}{l}z^{3} x^{2}+z x^{3} y+a z^{4} y^{3}+b z^{2} x y^{4}+ \\
c z y^{8}+d x^{2} y^{5},|a d|+|b|+|c| \neq 0\end{array}$ & $\begin{array}{l}t+g(z, x, y) \subset \mathbb{P}(29,5,7,3) \\
\mathbb{P}(5,7,3), \operatorname{Diff}=\left(\frac{1}{2}, 0,0,0\right)\end{array}$ & 6 \\
\hline 53 & $z^{3} x^{2}+x^{3} y^{2}+y^{10}$ & $\begin{array}{c}t^{2}+z x^{2}+x^{3} y^{2}+y^{10} \subset \mathbb{P}(15,14,8,3) \\
\text { Diff }=\left(0, \frac{2}{3}, 0,0\right)\end{array}$ & 7 \\
\hline 54 & $\begin{array}{c}z^{3} x^{2}+x^{3} y^{2}+y^{n} \\
n=7 ; 9 ; 13\end{array}$ & $\begin{array}{c}t+z x^{2}+x^{3} y^{2}+y^{n} \subset \mathbb{P}(3 n, n+4, n-2,3) \\
\mathbb{P}(n+4, n-2,3), \text { Diff }=\left(\frac{1}{2}, \frac{2}{3}, 0,0\right)\end{array}$ & $9,12,18$ \\
\hline 55 & $\begin{array}{c}z^{3} x^{2}+x^{3} y^{2}+a z^{6} y+b z^{3} y^{6}+ \\
c z^{3} x y^{3}+d x^{2} y^{5}+e x y^{8}+l y^{11} \\
|a d|+|b|+|c|+|e|+|l| \neq 0\end{array}$ & $\begin{array}{c}t+g\left(z^{1 / 3}, x, y\right) \subset \mathbb{P}(11,5,3,1) \\
\mathbb{P}(5,3,1), \text { Diff }=\left(\frac{1}{2}, \frac{2}{3}, 0,0\right)\end{array}$ & 6 \\
\hline 56 & $z^{3} x^{2}+x^{3} y^{2}+y^{12}$ & $\begin{array}{c}t^{2}+z x^{2}+x^{3} y+y^{6} \subset \mathbb{P}(9,8,5,3) \\
\text { Diff }=\left(0, \frac{2}{3}, 0, \frac{1}{2}\right)\end{array}$ & 9 \\
\hline 57 & $\begin{array}{c}z^{3} x^{2}+x^{3} y^{2}+a z^{3} y^{n-2}+ \\
b x y^{n}, n=5 \quad a \neq 0 ; 9\end{array}$ & $\begin{array}{l}t+g\left(z^{1 / 3}, x, y\right) \subset \mathbb{P}(3 n-2, n+2, n-2,2) \\
\quad \mathbb{P}(n+2, n-2,2), \text { Diff }=\left(\frac{1}{2}, \frac{2}{3}, 0,0\right)\end{array}$ & 6,12 \\
\hline $\begin{array}{l}58 \\
(1)\end{array}$ & $\begin{array}{c}z^{3} x^{2}+x^{3} y^{2}+a z^{5} y^{2}+b z^{4} x y+ \\
c z^{3} y^{5}+d z^{2} x y^{4}+e z x^{2} y^{3}+ \\
l z y^{8}+n x y^{7}\end{array}$ & $\begin{array}{l}t+g(z, x, y) \subset \mathbb{P}(19,3,5,2) \\
\mathbb{P}(3,5,2), \text { Diff }=\left(\frac{1}{2}, 0,0,0\right)\end{array}$ & 4 \\
\hline 59 & $\begin{array}{c}z^{3} x^{2}+x^{3} y^{2}+z y^{n} \\
n=5 ; 7 ; 9 ; 11\end{array}$ & $\begin{array}{c}t+g(z, x, y) \subset \mathbb{P}(9 n+4, n+4,3 n-4,8) \\
\mathbb{P}(n+4,3 n-4,8), \text { Diff }=\left(\frac{1}{2}, 0,0,0\right)\end{array}$ & $\begin{array}{c}7,10,14 \\
16\end{array}$ \\
\hline 60 & $\begin{array}{c}z^{3} x^{2}+x^{3} y^{2}+a z^{2} x y^{n}+ \\
b z y^{2 n}, n=3 ; 5\end{array}$ & $\begin{array}{c}t+g(z, x, y) \subset \mathbb{P}(9 n+2, n+2,3 n-2,4) \\
\mathbb{P}(n+2,3 n-2,4), \text { Diff }=\left(\frac{1}{2}, 0,0,0\right)\end{array}$ & 6,8 \\
\hline 61 & $\begin{array}{c}z^{3} x^{2}+x^{3} y^{2}+a z^{4} y^{2}+b z x y^{4} \\
a b \neq 0\end{array}$ & $\begin{array}{c}t^{2}+g(z, x, y) \subset \mathbb{P}(17,6,8,5) \\
\text { Diff }=\varnothing\end{array}$ & 3 \\
\hline 62 & $\begin{array}{c}z^{3} x^{2}+x^{3} y^{2}+a z^{4} y^{n-2}+ \\
b z x y^{n}, n=5 ; 7\end{array}$ & $\begin{array}{c}t+g(z, x, y) \subset \mathbb{P}(9 n-2, n+2,3 n-4,5) \\
\mathbb{P}(n+2,3 n-4,5), \text { Diff }=\left(\frac{1}{2}, 0,0,0\right)\end{array}$ & 8,10 \\
\hline 63 & $\begin{array}{c}z^{3} x^{2}+x^{3} y^{2}+a z^{4} y^{4}+ \\
b z x y^{6}\end{array}$ & $\begin{array}{c}t^{2}+g\left(z, x, y^{1 / 2}\right) \subset \mathbb{P}(13,4,7,5) \\
\text { Diff }=\left(0,0,0, \frac{1}{2}\right)\end{array}$ & 5 \\
\hline 64 & $\begin{array}{c}z^{3} x^{2}+x^{3} y^{2}+z^{2} y^{4 n} \\
n=1 ; 2\end{array}$ & $\begin{array}{c}t^{2}+g\left(z, x, y^{1 / 2}\right) \subset \mathbb{P}(9 n+2,2 n+2,6 n-1,7) \\
\text { Diff }=\left(0,0,0, \frac{1}{2}\right)\end{array}$ & 6,7 \\
\hline 65 & $\begin{array}{c}z^{3} x^{2}+x^{3} y^{2}+z^{2} y^{n} \\
n=5 ; 7 ; 9\end{array}$ & $\begin{array}{c}t+g(z, x, y) \subset \mathbb{P}(9 n+8, n+4,3 n-2,7) \\
\mathbb{P}(n+4,3 n-2,7), \text { Diff }=\left(\frac{1}{2}, 0,0,0\right)\end{array}$ & $8,12,14$ \\
\hline 66 & $z^{3} x^{2}+x^{3} y^{2}+z^{2} y^{6}$ & $\begin{array}{c}t^{2}+g(z, x, y) \subset \mathbb{P}(31,10,16,7) \\
\text { Diff }=\varnothing\end{array}$ & 5 \\
\hline
\end{tabular}

(1) Условие на исключительность: $|c|+|d|+|l|+|n|+|e(|a|+|b|)| \neq 0$. 


\section{Список литературы}

1. Арнольд В. И., Варченко А. Н., Гусейн-Заде С. М. Особенности дифференцируемых отображений. I. М.: Наука, 1982.

2. Iano-Fletcher A. R. Working with weighted complete intersections // Explicit birational geometry of 3-folds. London Math. Soc. Lecture Note Ser. V. 281. Cambridge: Cambridge Univ. Press, 2000. P. 73-173,.

3. Fujino $O$. The indices of log canonical singularities. e-print math.AG/9909035.

4. Ishii S. On isolated Gorenstein singularities // Math. Ann. 1985. V. 270. P. 541-554.

5. Ishii $S$. The quotient of log-canonical singularities by finite groups // Advanced Studies in Pure Math. 2000. V. 29. P. 135-161.

6. Kawamata $Y$. Crepant blowin-ups of 3-dimensional canonical singularities and its application to degenerations of surfaces // Ann. Math. 1988. V. 127. P. 93-163.

7. Kawamata Y., Matsuda K., Matsuki K. Introduction to the minimal model program // Sendai. Adv. Stud. Pure Math. 1987. V. 10. P. 283-360.

8. Kollar J. et al Flips and abundance for algebraic threefolds // Astérisque. 1992. V. 211.

9. Kollár J. Singularities of pairs // Proc. Symp. Pure Math. 1995. V. 62. P. 221-287.

10. Кудрявиев C. А. Об исключительных терминальных особенностях // УМН. 2001. Т. 56. № 1. C. $165-166$.

11. Кудрявиев С. А. О чисто логтерминальных раздутиях // Матем. заметки. 2001. Т. 69. №6. C. $892-899$.

12. Lazzeri F. On the monodromy of isolated singularities // Astérisque. 1973. V. 78. P. 269-276.

13. Маркушевич Д. Г. Канонические особенности трехмерных гиперповерхностей // Изв. АН CCCP. Сер. матем. 1985. Т. 49. С. 334-368.

14. Oda T. Convex bodies and algebraic geometry. An introduction to the theory of toric varieties. Berlin-Heidelberg-N. Y.-London-Paris-Tokyo: Springer-Verlag, 1988.

15. Прохоров Ю.Г. О дополняемости канонического дивизора для расслоений Мори на коники // Матем. сб. 1997. Т. 188. №11. С. 99-120.

16. Prokhorov Yu. G. Mori conic bundles with a reduced log-terminal boundary // J. Math. Sci. 1999. V. 91. P. 1051-1059.

17. Prokhorov Yu. G. Blow-ups of canonical singularities // Algebra. Proc. Internat. Conf. on the Occasion of the 90th birthday of A. G. Kurosh. Moscow, Russia, May 25-30, 1998 / Ed. Yu. Bahturin. Berlin: Walter de Gruyter, 2000. P. 301-317.

18. Prokhorov Yu. G., Markushevich D. G. Klein's group defines an exceptional singularity of dimension 3 // J. Math. Sci. 1999. V. 91. P. 1060-1067.

19. Ishii S., Prokhorov Yu. G. Hypersurface exceptional singularities // Intern. J. of Math. 2001. V. 12. № 6. P. 661-687.

20. Prokhorov Yu. G. Lectures on complements on log surfaces // MSJ Memoirs. 2001. V. 10.

21. Reid M. Canonical threefolds // Gáométrie Algébrique Angers / Ed. A. Beaville. Paris: Sijthoff and Noordhoff, 1980. P. 273-310.

22. Reid M. Young person's guide to canonical singularities // Proc. Symp. in Pure Math. 1987. V. 46. P. 343-416.

23. Шокуров В. В. Трехмерные логперестройки // Изв. АН СССР. Сер. матем. 1992. Т. 56. C. $105-203$.

24. Shokurov V. V. Complements on surfaces // J. of Math. Sci. 2000. V. 102. № 2. P. 3876-3932.

25. Varchenko A.N. Zeta-function of monodromy and Newton's diagram // Invent. Math. 1976. V. 37. P. 253-262.

26. Yonemura T. Hypersurface simple K3-singularities // Tohoku Math. J. 1990. V. 42. P. 351-380.

27. Кудрявцев $C$. A. Классификация трехмерных исключительных логканонических гиперповерхностных особенностей. II // Тр. МИАН. 2002; e-print math.AG/0202105.

Московский государственный университет им. М. В. Ломоносова Поступило в редакцию 